\title{
Medizinische Rehabilitation
}

herausgegeben von

o.Univ.-Prof. Dr. Peter Jabornegg ao.Univ.-Prof. Dr. Reinhard Resch

Univ.-Prof. Dr. Otfried Seewald 
Zitiervorschlag: Autor in Jabornegg/Resch/Seewald (Hrsg), Medizinische Rehabilitation (2009) [Seite]

\section{Printed in Austria}

Alle Rechte, insbesondere das Recht der Vervielfältigung und Verbreitung sowie der Ubersetzung, vorbehalten. Kein Teil des Werkes darf in irgendeiner Form (durch Fotokopie, Mikrofilm oder ein anderes Verfahren) ohne schriftliche Genehmigun des Verlages reproduziert oder unter Verwendung elektronischer Systeme gespeichert, verarbeitet, vervielfältigt oder verbreitet werden.

Sämtliche Angaben in diesem Werk erfolgen trotz sorgfältiger Bearbeitung ohne Gewähr eine Haftung der Herausgeber, der Autoren sowie des Verlages ist ausgeschlossen.

ISBN 978-3-214-09342-6

\section{Vorwort}

Fragen der medizinischen Rehabilitation in der gesetzlichen Krankenversicherung stellen sich an der Schnittstelle von Medizin und Rechtswissenschaft. Bereits die richtige medizinische Einordnung in der Medizin ist nicht immer exakt möglich. Die Einbettung der medizinischen Rehabilitation in das Rech der gesetzlichen Sozialversicherung erwies sich als schwierig, weisen doch verschiedene Sparten der gesetzlichen Sozialversicherung Berührungspunkte zur medizinischen Rehabilitation auf, sodass durchaus verschiedene Träger für die Leistungserbringung zuständig sein könnten. Dem Schnittstellenmanagemen zwischen den potentiell zuständigen Trägern kommt damit maßgebliche rechtliche und ökonomische Bedeutung zu.

Die 11. Deutsch-Österreichischen Sozialrechtsgespräche (29. und 30.1. 2009) widmen sich - in dieser Tiefe erstmals - der wissenschaftlichen Durchdringung des Rechts der medizinischen Rehabilitation und bringen dazu den interessanten Rechtsvergleich zwischen Deutschland und Österreich.

Die Herausgeber schulden der Oberösterreichischen Gebietskrankenkasse für die maßgebende Unterstützung der Tagung und dieses Tagungsbandes Dank.

Peter Jabornegg Reinhard Resch Otfried Seewald

C 2009 MANZ'sche Verlags- und Universitätsbuchhandlung GmbH, Wien Telefon: (01) 531 61-0

$$
\text { eMail: verlag@MANZ.at }
$$

World Wide Web: www.MANZ.

Datenkonvertierung, Satzherstellung: Druckerei Robitschek \& Co. Ges.m.b.H., 1050 Wien

Druck: Börsedruck Ges.m.b.H., 1230 Wien 


\section{Inhaltsverzeichnis}

Vorwort

Autorenverzeichnis

Abkürzungsverzeichnis

Gottfried Endel

(Medizinische) Prozessbeschreibung aus Sicht der österreichischen Sozialversicherung

1. Abstract

2. Definition: Rehabilitation / Krankenbehandlun

2.1 Medizinische Definitionen

2.2 Rechtliche Definition

2.3 Organisatorische Definition

3. Beispiel: Myocardinfarkt

3.1 Zeitablauf

3.2 Zusammenfassung: Beispiel Myocardinfark

4. Zusammenfassung

Roland Benkowitsch

Prozessbeschreibung der medizinischen Rehabilitation aus deutscher Sich

1. Abgrenzung Kuration - Rehabilitation

. Grundlagen "Medizinische Rehabilitation"

3. Medizinische Voraussetzungen der Rehabilitation

4. "Interessen" an der Rehabilitationsbehandlung

5. Das Phasen-Modell in der Neurologie

6. Einordnung der geriatrischen Rehabilitation

7. Begriff der Frührehabilitation

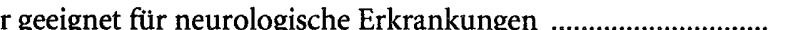

9. Informationsquellen

10. Nachhaltigkeit von Rehabilitation - Praxisschock

11. Sammlung von Fallbeispielen der medizinischen Rehabilitation

11.1 Erster Normalfall: Abfolge Akutbehandlung - Rehabilitation

11.2 Zweiter Normalfall: Abfolge Akutbehandlung - Frührehabilitation .......... 28

11.3 Versorgungsproblem: Mangelnde Rehabilitation während Akutbehandlung

11.4 Versorgungsproblem: Fehler in der Akutbehandlung - dadurch hoher Rehabilitationsaufwand

11.5 Abgrenzungsproblem: Akutbehandlung - stationäre - ambulante Rehabilitation

11.6 Abgrenzungsproblem: Rehabilitation Phase $\mathrm{C}-\mathrm{D}$-ambulant 
11.7 Abgrenzungsproblem: stationäre bzw ambulante Rehabilitation ................ 31 Rehabilitation

12. Zusammenfassung

\section{Johannes Rudda}

Das System der Rehabilitation in Österreich

. Das System der Rehabilitation in Österreich ............................................................. 33

1.1 Historische Entwicklung .................................................................................... 33

1.2 Einrichtungen der Sozialversicherung und privater Rechtsträger ................... 34

1.3 Gesetzliche Ermächtigung zu privatrechtlichen Beteiligungsmöglichkeiten . 34

1.4 Die Rehabilitation im Stufenbau der Rechtsordnung ..................................... 36

1.4.1 Rehabilitationsleistungen der Unfallversicherung ................................... 36

1.4.2 Rehabilitationsleistungen der Pensionsversicherung ................................. 37

1.4.3 Rehabilitationsleistungen der Krankenversicherung …........................ 37

1.4.4 Rehabilitations- und Gesundheitsvorsorgerichtlinien

des Hauptverbandes

5 Grundsätze für die medizinische Rehabilitation ............................................. 39

1.5.1 Stationäre oder ambulante Rehabilitation ............................................... $\quad 39$

1.5.2 Rehabilitation vor Pension .................................................................... 40

1.5.3 Grundsatz der Rehabilitationsfähigkeit ................................................. 40

2. Begriffliche Abgrenzung von anderen Maßnahmen der Rehabilitation ................. 41

2.1 Berufliche Maßnahmen der Rehabilitation ...................................................... 41

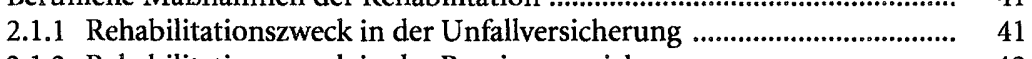

2.1.2 Rehabilitationszweck in der Pensionsversicherung …........................... 42

2.1.3 Begriffliche Abgrenzung der beruflichen Rehabilitation zur medizinischen Rehabilitation

2 Soziale Maßnahmen der Rehabilitation .............. 42

2.2.1 Soziale Maßnahmen der Rehabilitation in der Unfallversicherung ..... $\quad 42$

2.2.2 Soziale Maßnahmen der Rehabilitation in der Pensionsversicherung .. 43

2.2.3 Soziale Krankenversicherung ..........

2.3 Besonderheiten der beruflichen und sozialen Rehabilitation in den Sondergesetzen

3.1 Die Leistungen der medizinischen Rehabilitation im Überblick bei der

Unfallversicherung

3.1.1 Die medizinische Rehabilitation und der Eintritt des

Versicherungsfalls

3.1.2 Parallelleistungen der medizinischen Rehabilitation zur Unfallheilbehandlung

3.1.3 Leistungserbringer und -träger der medizinischen Rehabilitation in der Unfallversicherung

3.2 Die Leistungen der medizinischen Rehabilitation im Überblick bei der

Pensionsversicherung .......................................................................... Die Leistungserbringer der medizinischen Rehabilitation in der Pensionsversicherung

3.2.2 Leistungsträger der medizinischen Rehabilitation in der Pensionsversicherung

3.3 Die Leistungen der medizinischen Rehabilitation in der Krankenversicherung .
3.3.1 Leistungserbringer der medizinischen Rehabilitation in der Krankenversicherung

3.3.2 Leistungsträger in der medizinischen Rehabilitation der Krankenversicherung

4. Materielle Abgrenzung zu anderen Leistungsbereichen des Versorgungssystems ... 50

4.1 Medizinische Rehabilitation versus Krankenbehandlung ............................... 50

4.2 Abgrenzung zur Anstaltspflege bei Akuterkrankung .................... 50

4.3 Abgrenzung zur medizinischen Hauskrankenpflege ......................................... 51

4.4 Medizinische Rehabilitation und die Gewährung von Heilbehelfen und Hilfsmitteln

4.5 Medizinische Rehabilitation und Pflegebedürftigkeit nach $\$ \$ 4$ und 4a BPGG

5. Rechtsprobleme und Systemkritik .....................................................................

5.1 Generelle Defizite in der medizinischen Rehabilitation .................................. 52

5.2 Bedarf an Case Managern zur Überwindung individueller Lücken .................. 53

5.3 Rechts- und Zuständigkeitsprobleme ............................................................ 53

5.4 Notwendigkeit einer stärkeren Kooperation bei gleicher Qualität

5.5 Aufbau eines gezielten Netzwerks für alle Rehabilitationsfälle ......................... 54

Otfried Seewald

Medizinische Rehabilitation - die Rechtslage im deutschen SV-Recht

1. Einleitung

2.1 Trennung von „Akutversorgung" und Rehabilitation ........................................ 58

2.2 Fragwürdigkeit der Trennung ..........................

2.3 Fehlen eines Rehabilitations- „Grundtatbestandes “ .......................................... 59

2.4 Die Situation in der gesetzlichen Unfallversicherung .................................... 60

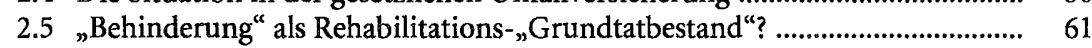

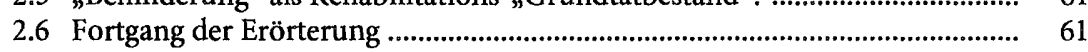

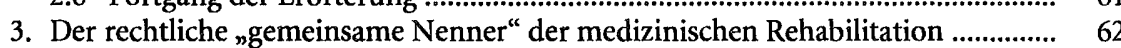

3.1 Die Rechtsgrundlage im SGB IX ............................................................... 62

3.2 Materielles Recht - ein Leistungsfall "der“ medizinischen Rehabilitation? ... 62

3.3 Wirkung des SGB IX in den Besonderen Teilen des SGB ................................ 63

3.4 Einzelfragen zu $\$ 26$ SGB IX .................................................................................

3.4.1 Konkretisierung unbestimmter Gesetzesbegriffe durch Verwaltung und Gerichte. Das Anliegen des SGB IX

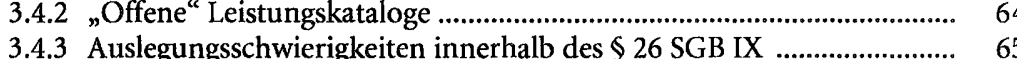

.

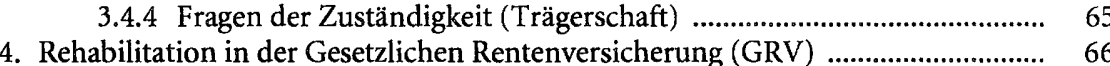

4.1 Der Standort der medizinischen Rehabilitation in der GRV

4.2 Die Bedeutung der „Behinderung“ im SGB VI und im Vergleich mit dem SGB IX

4.3 Die Bedeutung der..................................................... "Grundtatbeständen" des SGB VI

4.4 Tatbestandliche Voraussetzungen und Wirkungsbreite der des SGB IX .......

5 Die rehabilitationsrechtlichen Regelungen des SGB VI

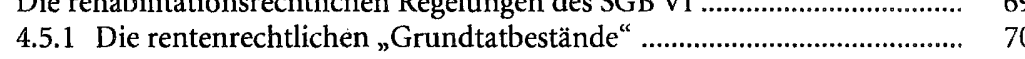

4.5.2 Drohende Auswirkungen auf die Erwerbsfähigkeit ...................................... 
4.5.3 Gemeinsames Merkmal der „Erheblichkeit“

Tatbestandsbezogener Vergleich zwischen SGB VI und SGB IX

$\begin{array}{ll} & \end{array}$

4.6.2 Die Bedeutung der Behinderung

4.7 Die Leistungen der medizinischen Rehabilitation.

Vergleich zwischen SGB IX und SGB VI

X ..................................... 74

(1)

4.7.3 Weitere Zielgruppen ................................................................................. 75

4.7.4 Behandlungen .................................................................................... 75

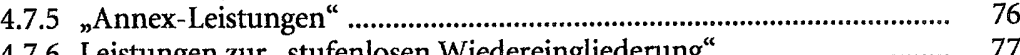

4.7.6 Leistungen zur „stufenlosen Wiedereingliederung

4.7.7 Förderung der Selbsthilfe

4.7.8 Allgemeine Regelungen des SGB IX für die Leistungen zur

medizinischen Rehabilitation

(

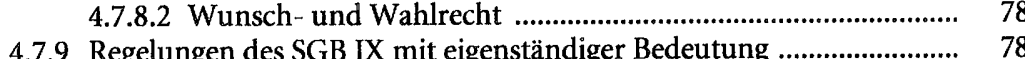

4.8 Gestaltungsfreiheit der Verwaltung (Ermessen / Beurteilungsspielraum) ...... 79

4.9 Gesetzliche Beschränkungen des Leistungsumfanges / Rationierung von

Leistungen zur medizinischen Rehabilitation / Leistungsumfang .................. 80

5. Medizinische Rehabilitation in der gesetzlichen Krankenversicherung .................. 80

5.1 Änderungen in der systematischen Zuordnung ...

5.2 Überblick über die Regelungen im SGB V .................................................... 81

5.2.1 Anspruchsgrundlagen zu den „Leistungen zur medizinischen

Rehabilitation"

5.2.1.1 „Ergänzung" der ambulanten Krankenbehandlung ......................

5.2.12 Frührehabilitation

5.22 Anschlussrehabilitation .

5.3 Medizinische Rehabilitation für Mütter und Väter ……………….........................

5.4 Belastungserprobung und Arbeitstherapie ........................................................ 83

5.5 Ergänzende Leistungen zur Rehabilitation ...................................................... 83

5.5.1 Medizinische Rehabilitation ....

5.5.2 Sozialmedizinische Nachsorgemaßnahmen ......................................... 84

5.5.3 Weitere Leistungen ............................................................................... 85

5.6 Gestaltungsfreiheit der Verwaltung (Ermessen / Beurteilungsspielraum) ..... 85

5.7 Verhältnis zu anderen Bereichen (Rentenversicherung, Unfallversicherung) ... 86

5.7.1 Medizinische Rehabilitation neben der ambulanten Krankenbehandlung

5.7.2 Ausnahme: $\$ 31$ SGB VI ................................................................. 87

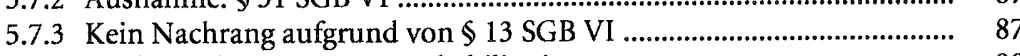

5.7.4 Nachrang bei stationärer Rehabilitation ............................................ $\quad 88$

5.7.5 Leistungen an pflegebedürftige Versicherte ..

6. Medizinische Rehabilitation in der gesetzlichen Unfallversicherung ..................... 89

6.1 Ganzheitliche „Heilbehandlung " .................................................................. 89

6.2 Das Leistungsniveau ........................................................................................ 8

6.3 Der Umfang der Heilbehandlung ............................................................ 90

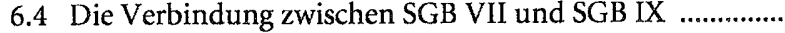

6.5 Die Durchführung der Heilbehandlung ……................................................ 90

6.6 Die Verfahrenstypen der unfallversicherungsrechtlichen Heilbehandlung ... 9

6.7 Keine rationierende Leistungsbegrenzung …………………….......................... 92
6.8 Verletztengeld bei medizinischer Rehabilitation

6.9 Vorrang / Nachrang der Leistungspflicht ..... 6.10 Ergänzende Leistungen

7. Medizinische Rehabilitation in der Pflegeversicherun

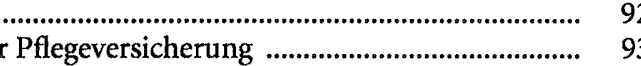

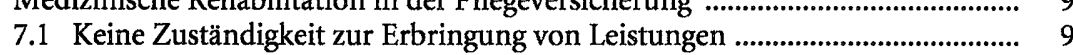

7.2 Ausnahme: Vorläufige Leistungen ............................................................. 93

7.3 Pflichten der Rehabilitationsträger im Hinblick auf Pflegebedürftigkeit ....... 94

8. Organisationsrechtliche Regelungen. Probleme und Entwicklungen ...................... 95

9. Organisatorische Lösungen der Kollisionen im Bereich der medizinischen

Rehabilitation

.

9.2.1 Die einzelnen Problemfelder 96

9.2.2 Verfahrensrechtliche Koordinierung zur Abstimmung des Leistungsgeschehens 9.2.2. Bildung IX - Koordinierung der Leistungen ........................ 98

9.2.2.3 $\$ 14$ SGB IX - Zuständigkeitsklärung

9.2.2.4 Ausführung der Leistungen durch ein persönliches Budget (\$17 Abs 2 SGB IX) 9.2.2.5 $\$ 15$ SGB IX - Erstattung selbst beschaffter Leistungen ... ............. 101 . 9.2.3.2 Qualitatssicherung .................................................................. 103 9.2.3.3 Verträge mit Leistungserbringern .......................................... 103

9.2.4 „Aufbauorganisatorische Ergänzungen“ zur Kooperation der Leistungsträger ...

9.2.4.1 Gemeinsame Servicestellen

9.2.4. Die Bundesarbeitsgemeinschaft für Rehabilitation

9.2.4.3 Weitere Bundesarbeitsgemeinschaften ..................................... 104

Anhang

Medizinische Rehabilitation im Sozialversicherungsrecht

- „Rehabilitationsrecht“ -

Auszüge aus dem Sozialgesetzbuch (SGB)

Reinhard Resch

Medizinische Rehabilitation in Österreich: Leistungsrecht und

Berührungspunkte zum Arbeitsrecht

1. Einleitung

2. Anspruch auf medizinische Rehabilitation?

22 Ermesentung im überschneidenden Bereich

3. Geld- und Sachleistungen im Zusammenhang mit Rehabilitation ........................

3.1 Teilversicherung in der gesetzlichen Unfallversicherung ............................. 141

3.2 Geldleistungen während Maßnahmen der medizinischen Rehabilitation? .... 142 3.2.1 Beruicksichtigung bei der Pflegegeldbemessung ................................... 142

3.2.2 Krankengeld .......................................................................................... 143

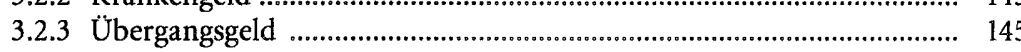


3.2.4 Zuschuss an den Dienstgeber gem $\$ 53 \mathrm{~b}$ ASVG?

3.2. Relevan Rehabilitation für

den Anspruch auf eine Pension wegen geminderter Arbeitsfähigkeit ... 146

4. Arbeitsrechtliche Fragen

4.1 Entgeltfortzahlung durch den Arbeitgeber ...

4.2 Schicksal des aufrechten Arbeitsvertrags bei Auslaufen de

Entgeltfortzahlung

\section{Autorenverzeichnis}

................................. 149

4.3.1 Behinderung iSd \$ 3 BEinstG .......................................................... 149

4.3.2 Gerechtfertigte oder unberechtigte Entlassung? .................................... 15

4.3.3 Kündigungs- und Entlassungsschutz ..................................................... 152

5. Zusammenfassung ….............................................................................................

\section{Hans Peter Spiegl}

Ausgewählte leistungsrechtliche Probleme im Zusammenhang mit Rehabilitation

- ausgewählte leistungsrechtliche Probleme in Deutschland

1. Einleitung

2. Anspruch auf Rehabilitation

3. Leistungsrechtliche Probleme in der Krankenversicherung ......... 156

4. Leistungsrechtliche Probleme in der Rentenversicherung ........................................ 160

5. Leistungsrechtliche Probleme in der UV ................................................................. 162

6. Geld- und Sozialleistungen ................................................................................ 164

7. Zuzahlungsregelungen ....................................................................................... 167

8. Mitwirkungsobliegenheit und Zumutbarkeit ........................................... 168

9. Entgeltfortzahlung durch den Arbeitgeber während Maßnahmen der medizinischen Rehabilitation

Bernhard Albert

Schnittstellenmanagement bei medizinischer Rehabilitation in Österreich

2. Zustandigkeit

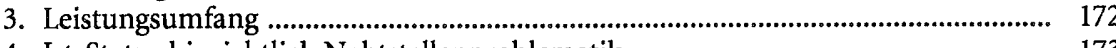

4. Ist-Status hinsichtlich Nahtstellenproblematik ........................................................... 173

5. Ideen für die Zukunft .............................................................................................. 175

6. Zusammenfassung ........................................................................................................... 176

\section{Eckart Stevens-Bartol}

\section{Schnittstellen im Recht der Rehabilitation in Deutschland}

1. Einleitung

2. Die Probleme des Zusammenwirkens ....................................................................

3. Der Begriff der Behinderung …........................................................................... 188

\author{
Dr. Bernhard Albert, MSc \\ Verwaltungsdirektor des Unfallkrankenhauses Linz \\ Garnisonstrasse 5 \\ 4020 Linz
}

\section{Dr. Roland Benkowitsch}

AOK Bayern - Die Gesundheitskasse, Dienstleistungszentrum

Im Gewerbepark B 32

D-93059 Regensburg

\section{Dr. Gottfried Endel}

Evidence Based Healthcare

Hauptverband der Österreichischen Sozialversicherungsträger

Kundmanngasse 21

1030 Wien

Prof. Dr. Johannes Rudda

Referatsleiter im Hauptverband der Österreichischen Sozialversicherungsträger Kundmanngasse 21

1030 Wien

ao.Univ.-Prof. Dr. Reinhard Resch

Institut für Arbeitsrecht und Sozialrecht

Universität Linz

Altenbergerstrasse 69

4040 Linz

Univ.-Prof. Dr. Otfried Seewald

Universität Passau

Schärdinger Strasse 23a

D-94032 Passau

\section{Hans Peter Spiegl}

Vorsitzender Richter des Bayerischen Landessozialgerichts

Schubertstraße 25

D-82049 Pullach

\section{Eckart Stevens-Bartol}

Vorsitzender Richter am Bayerischen Landessozialgericht a.D., Rechtsanwalt Adelgundenstraße 2

D-80538 München 


\section{Abkürzungsverzeichnis}

\begin{tabular}{|c|c|c|}
\hline $\mathrm{aA}$ & $=$ & anderer Ansicht \\
\hline $\mathrm{aaO}$ & $=$ & am angegebenen Ort \\
\hline ABGB & $=$ & Allgemeines bürgerliches Gesetzbuch \\
\hline Abs & $=$ & Absatz \\
\hline $\mathrm{aE}$ & $=$ & am Ende \\
\hline AG & $=$ & Arbeitgeber \\
\hline AlVG & $=$ & Arbeitslosenversicherungsgesetz \\
\hline AMS & $=$ & Arbeitsmarktservice \\
\hline AN & $=$ & Arbeitnehmer \\
\hline AngG & $=$ & Angestelltengesetz \\
\hline Anm & $=$ & Anmerkung \\
\hline AOK & $=$ & Allgemeine Ortskrankenkasse \\
\hline AP & $=$ & $\begin{array}{l}\text { Arbeitsrechtliche Praxis, Sammlung der Entscheidungen des } \\
\text { (deutschen) Bundesarbeitsgerichts, der Landesarbeitsgerichte } \\
\text { und Arbeitsgerichte }\end{array}$ \\
\hline ARÄG & $=$ & Arbeitsrechtsänderungsgesetz \\
\hline ARD & $=$ & Arbeitsrechtlicher Betriebsdienst \\
\hline Art & $=$ & Artikel \\
\hline ASG & $=$ & Arbeits- und Sozialgericht \\
\hline ASGG & $=$ & Arbeits- und Sozialgerichtsgesetz \\
\hline ASoK & $=$ & Arbeits- und Sozialrechtskartei \\
\hline ASVG & $=$ & Allgemeines Sozialversicherungsgesetz \\
\hline ATL & $=$ & Aktivitäten des täglichen Lebens \\
\hline AUVA & $=$ & Allgemeine Unfallversicherungsanstalt \\
\hline BAG & $=$ & Bundesarbeitsgericht \\
\hline BAR & $=$ & Bundesarbeitsgemeinschaft für Rehabilitation \\
\hline BBG & $=$ & Bundesbehindertengesetz \\
\hline BEinstG & $=$ & Behinderteneinstellungsgesetz \\
\hline BGBl & $=$ & Bundesgesetzblatt \\
\hline B-KUVG & $=$ & Beamten-Kranken- und Unfallversicherungsgesetz \\
\hline BlgNR & $=$ & Beilagen zu den stenografischen Protokollen des Nationalrats \\
\hline BMSVG & $=$ & Betriebliches Mitarbeiter- und Selbständigenvorsorgegesetz \\
\hline BPGG & $=$ & Bundespflegegeldgesetz \\
\hline BRD & $=$ & Bundesrepublik Deutschland \\
\hline BSB & $=$ & $\begin{array}{l}\text { Bundessozialamt für die Gleichstellung und Integration von } \\
\text { Menschen mit Behinderung }\end{array}$ \\
\hline BSG & $=$ & Bundessozialgericht \\
\hline BSGE & $=$ & Entscheidungen des Bundessozialgerichts \\
\hline BSHG & $=$ & Bundessozialhilfegesetz \\
\hline bspw & $=$ & beispielsweise \\
\hline BSVG & $=$ & Bauern-Sozialversicherungsgesetz \\
\hline BT & $=$ & Bundestag \\
\hline
\end{tabular}




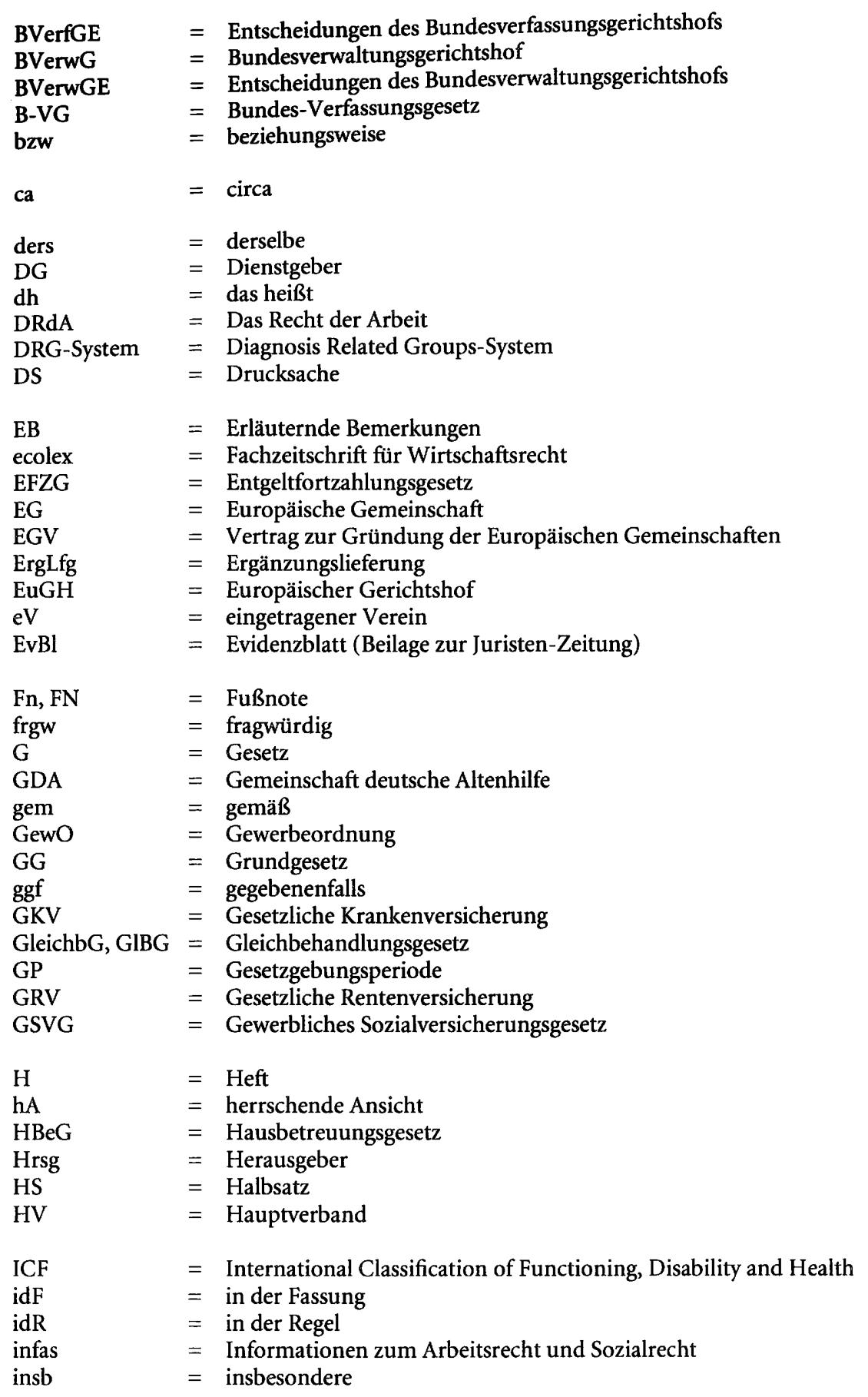

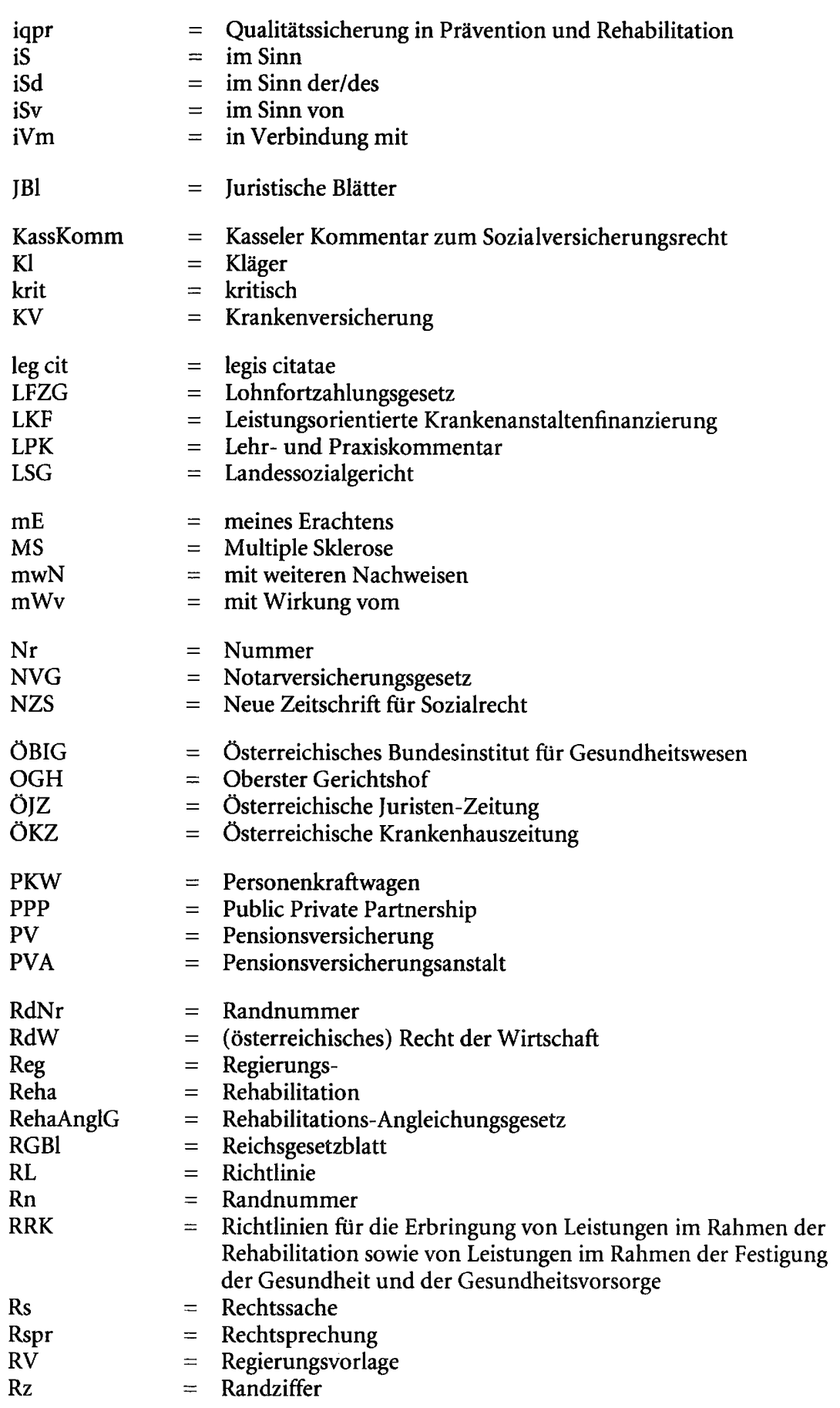




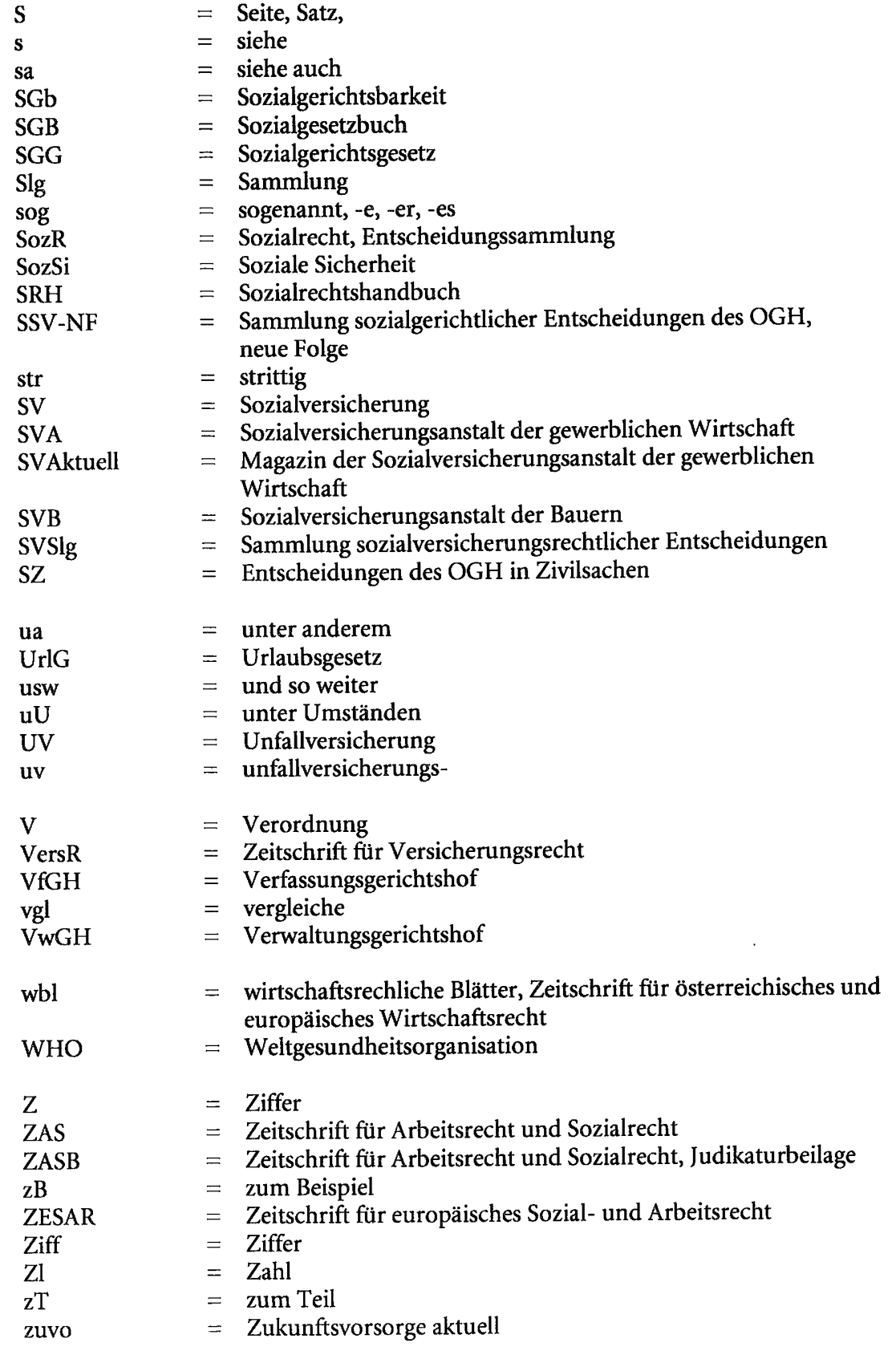

\section{Gottfried Endel}

\section{(Medizinische) Prozessbeschreibung aus Sicht der österreichischen Sozialversicherung}

\section{Abstract}

Vor einer Beschreibung eines beispielhaften Ablaufes des Krankheitsfalles "Herzinfarkt" und der daran anschließenden Rehabilitation ist es notwendig die Begriffe der Rehabilitation und der Krankenbehandlung aus medizinischer und leistungsrechtlicher Sicht zu beschreiben. Damit soll nicht versucht werden, die rechtlichen Aspekte umfassend und abschließend zu beschreiben, aber jene Punkte, welche zur Diskussion des unterschiedlichen Verständnisses erforderlich sind, benötigen eine begriffliche Klärung zumindest ihrer Verwendung in diesem Referat.

Rehabilitationsmedizin fokussiert im Gegensatz zur kurativen Medizin auf die (gestörte) Funktion. Die kurative Medizin orientiert sich hingegen überwiegend an Organpathologien und Krankheits- Entitäten. Dies führt unter anderem zu einer differenten Nomenklatur und der Notwendigkeit, Funktionsdefizite multidisziplinär zu analysieren und zu behandeln. Ein weiteres wesentliches Merkmal der Rehabilitation stellt die klare Zielbeschreibung basierend auf einer Begutachtung und dem Vereinbaren dieses Rehabilitationszieles mit dem Rehabilitanden dar. Es bleibt nicht ihm allein überlassen, ob Rehabilitation als notwendig und zweckmäßig erachtet wird. Dies führt zu einer Veränderung der wahrzunehmenden Rollen und einer klaren Abgrenzung gegenüber der Vorgangsweise bei der Inanspruchnahme von Krankenbehandlung. Rehabilitation wird auch nur für eine beschränkte und vereinbarte Zeit durchgeführt. Danach muss festgestellt werden, ob die angestrebten Ziele erreicht wurden. Ist dies der Fall, so endet die Rehabilitation.

Im Verständnis der Patienten/Rehabilitanden, der Mediziner und Mitglieder anderer Gesundheitsberufe aber manchmal auch der Laien ist eine klare Abgrenzung zwischen verschiedenen Begriffen wie Rehabilitation, Kurheilverfahren und Krankenbehandlung dringend erforderlich. Die medizinischen, rechtlichen und organisatorischen Unterschiede sind geeignet eine klare Abgrenzung vorzunehmen. Der Einfluss auf die zu diesen unterschiedlichen Handlungsbereichen gehörenden Verhaltensweisen, sowie die Abfolge der Ausprägung im Alltag einer Krankheitsepisode soll in der Folge exemplarisch dargestellt werden.

Das Beispiel eines Herzinfarktes zeigt deutlich das Überwiegen der Krankenbehandlung in der Akutphase. Während der lebensbedrohlichen Einschränkungen der Handlungs- und Leistungsfähigkeit verliert eine Person den Großteil der alltäglichen Handlungskompetenz und erlebt ein „Ausgeliefertsein“ an das Behandlungssystem. Erst nach der Stabilisierung des körperlichen Zustandes 
kann über das „Danach“ und die erreichbaren Ziele gesprochen werden. Damit beginnt die Planung und Gestaltung der Rehabilitation, mit welcher die Wiedereingliederung in die bisherigen Lebensumstände angestrebt wird. Neben einer Wiederherstellung der körperlichen Leistungsfähigkeit wird auch die Belastbarkeit des Herz- Kreislaufsystems trainiert. Dies beginnt meist mit hoher Intensität und kann danach über einen längeren Zeitraum unterstützend fortgeführt werden. Dabei nimmt aber bereits der Stellenwert der Rehabilitation gegenüber der Krankenbehandlung wieder ab. Das Ende und damit der Übergang in die so genannte sekundäre Prävention, welche wiederum Krankenbehandlung darstellt obwohl die Maßnahmen identisch sind und nur die Verbindlichkeit und Zuständigkeit wechseln, ist nach dem anfangs geplanten Zeitraum und dem Erreichen der angestrebten Ziele erreicht.

Ergibt die Begutachtung am Ende der Rehabilitations-Periode bestehende funktionelle Defizite, so muss beurteilt werden, ob diese die Wiedereingliederung verhindern und mit weiteren Maßnahmen eine Aussicht auf deren Behebung besteht. Dann kann eine weitere Rehabilitationsperiode angeschlossen werden.

Zusammenfassend erscheint es als besonders wichtig alle handelnden Personen auf die Unterschiede zwischen Rehabilitation und Krankenbehandlung hinzuweisen, damit nicht unklares Rollenverständnis und falsche Erwartungen den Verlauf einer Krankheitsepisode belasten und komplizieren.

\section{Definition: Rehabilitation / Krankenbehandlung}

Zwei von mehreren möglichen Handlungsfeldern von Ärzten und anderen Anbietern von Gesundheitsdienstleistungen sind die Krankenbehandlung und die Rehabilitation. Diese werden häufig als konkurrierend aber auch als zeitlich nebeneinander oder nacheinander folgende Tätigkeitsbereiche verstanden. Vielfach erscheinen die verwendeten Methoden als gleich und es ist nicht einsichtig, warum einer der beiden Sichtweisen der Vorzug gegeben werden soll. Um hier eine klare Diskussion zu ermöglichen ist es erforderlich zuerst diese Begriffe und Inhalte zu definieren. Dabei ist auch ein kurzer Exkurs in die geschichtliche Entwicklung der Medizin, die Entwicklung rechtlicher Regelung und die Organisation im Österreichischen Sozialsystem hilfreich.

\subsection{Medizinische Definitionen}

Für die Entwicklung der Medizin hat der naturwissenschaftliche Fortschritt mit seiner strukturierten erkenntnistheoretischen Vorgangsweise einen enormen Fortschritt im Verständnis der Funktionen und Strukturen des menschlichen Körpers bewirkt. Diese neue Sichtweise des Organismus als „Maschine“ mit Einblick in Regulationsmechanismen auf unterschiedlicher Detaillierungsebene und in Strukturen von der grob-anatomischen bis zur submikroskopischen Ebene hat frühere Erklärungsversuche von Krankheit, wie beispielsweise das Gleichgewicht der Säfte, ersetzt und insbesondere bei der Behandlung akuter Krankheiten ein Spezialisierung auf Organsysteme ermöglicht. Diese „Organmedizin“ hat die Zielsetzung durch Krankheit gestörte Regulationsvorgänge (meist medikamentös) wieder auszugleichen und Organveränderungen - soweit reversibel - rückgängig zu machen oder häufiger ihr weiteres Fortschreiten zu verhindern. Krankheitsbedingte Organveränderungen sind dabei häufig nur bei Betrachtung der zeitlichen Entwicklung von altersbedingten „Degenerationen“ zu unterscheiden.

\section{Definition - medizinisch:}

Rehabilitation ist daher neben Prävention, Kuration und palliativer Medizin ein eigenes Gebiet mit einer eigenen Systematik und spezifischen Grundlagen. Das Ziel der Kuration ist die „Restitutio ad Integrum“, Ziel der Rehabilitation ist die „Restitutio ad Optimum“.

Diese Ausrichtung auf Organveränderungen, ihre zeitliche Entwicklung und die Beeinflussbarkeit dieser Abläufe sowie auf physiologische Regelungsmechanismen und ihre messbaren Kenngrößen (wie Blutdruck und Laborwerte) bestimmt derzeit die Ausbildung insbesondere im Medizinstudium aber auch von anderen Gesundheitsberufen. Ergebnisqualität wird meist anhand so genannte "harter Endpunkte" wie Tod oder Wiederauftreten eines akuten Zustandes gemessen.

\section{Das Ziel der „Kuration“ ist daher die „Restitutio ad Integrum“.}

Da im Laufe der Entwicklung dieser Ansatz zunehmend erfolgreich umgesetzt werden konnte, wurden auch die durch die Verengung des Zuganges entstehenden Defizite erkennbar. Eine Restitutio ad Integrum gelingt nur sehr selten und das Voranschreiten von Organveränderungen lässt sich auch durch Prävention und Krankenbehandlung nicht aufhalten sondern bestenfalls auf die Geschwindigkeit normalen Alterns verringern. Damit erleben mit steigender Lebenserwartung immer mehr Mitmenschen eine Lebenszeit zunehmenden (Organ-)Funktionsverlustes. Eine Lebensverlängerung um jeden Preis und in jedem Zustand wird von vielen eher als Bedrohung denn als Verheißung verstanden Als eine mögliche, "medizinische" Antwort auf diese Defizite ist eine Ausrichtung nach Behinderung, Aktivität und Teilhabe.

Definition - medizinisch:

In der Medizin bezeichnet Rehabilitation den Einsatz und die Wirkung von Maßnahmen, die darauf zielen, die körperlichen, psychischen und sozialen Folgen einer Behinderung bzw Aktivitätseinschränkung (früher: „Disability“, jetzt Acitvity) und Störung der Teilhabe (früher: „Handicap“, jetzt: Participation) auf ein Minimum zu beschränken.

Im Sozial- und Arbeitsleben bedeutet Rehabilitation heute die Wiedereingliederung in den Alltag oder das berufliche Leben.

Die eingesetzten Maßnahmen unterscheiden sich nicht wesentlich. Medizinische Behandlungen werden mit sozialen und beruflichen Interventionen kombiniert. Die Zielsetzung richtet sich allerdings primär nicht nach einer organspezifischen Spezialisierung, sondern nach den Funktionen, welche im Arbeitsleben und Alltag benötigt werden. Ärztliche Behandlung ist in dieser Kombination gleichberechtigt neben mehreren anderen Hilfestellungen. Je nach Aktivitätseinschränkung können Funktionsstörungen durch Hilfsmittel, durch Pflegemaßnahmen oder durch Hilfestellungen im Alltag ausgeglichen werden. 
Zur Reduktion von Behinderungen und eine Anpassung an die verbleibenden Einschränkungen sind umfangreiche Unterrichtseinheiten erforderlich. Auch der Umgang mit Hilfsmitteln und Heilbehelfen erfordert von den Betroffenen sehr viel Flexibilität.

Rückblickend lässt sich diese Entwicklung wohl am besten an der Unfallheilbehandlung erkennen. Arbeitsunfälle oder Kriegsverletzungen führten vor de Entwicklung der wissenschaftlichen kurativen Medizin häufig zum Tod. Waren die Verletzungen geringgradig so verblieb häufig doch eine beträchtliche Behinderung und führte zu Verelendung und stark eingeschränkten Möglichkeiten ein normales Leben zu führen. Fortschritte bei der Akutbehandlung ermöglichten für eine höhere Zahl von Betroffenen ein Überleben - allerdings als (im damaligen Sprachgebrauch) „Krüppel“.

Daher wurde bei der Entwicklung der Unfallchirurgie durch Lorenz Böhler bereits während der Akutbehandlung mit der Wiederherstellung oder Rehabilitation begonnen. In einer ganzheitlichen Sicht wurde während der Heilungsphase mit funktionellen Übungen begonnen und bei der operativen Versorgung bereits auf eine spätere prothetische Versorgbarkeit hingearbeitet. In späteren Heilungsphasen wurden neben der Stärkung durch funktionelle Übungen auch Schulungsmaßnahmen bis hin zur Berufsausbildung in einem neuen geeigneten "Behindertenberuf“ eingeplant. Unterstützungen und Arbeitsplatzadaptationen dienten dazu, den eigenen Beruf weiter ausüben zu können.

\section{Definition - medizinisch:}

Rehabilitation umfasst den koordinierten Einsatz medizinischer, sozialer, beruflicher, pädagogischer und technischer Maßnahmen sowie Einflussnahmen auf das Psychische und soziale Umfeld zur Funktionsverbesserung zum Erreichen einer größtmöglichen Eigenaktivität zur weitestgehend unabhängigen Partizipation in allen Lebensbereichen, damit der Betroffene in seiner Lebensgestaltung so frei wie möglich wird." (WHO Definition der Rehabilitation nach dem technical report 668/1981)

Vgl: http://www.boepmr.at/html/aezte/zeitung/2_02/allgemeine\%20

Rehabilitation.pdf

Die Definition der WHO löst sich von dieser ursprünglich stark auf Verletzungsfolgen ausgerichteten Sicht. Der Fortschritt der Organmedizin lässt $\mathrm{Pa}$ tienten immer mehr schwere Akuterkrankungen überleben und die höhere Lebenserwartung schafft Rehabilitationsbedarf für Senioren.

Definition - medizinisch:

Wiederherstellung, Eingliederung

Maßnahmen zur Vorbeugung oder Beseitigung von schweren gesundheitlichen Störungen.

Sozialversicherungsrechtlich erfolgt eine sehr restriktive Definition sowie Kostenübernahme und zwar im Sinne der beruflichen Wiedereingliederung.

Geriatrische Rehabilitation: Maßnahmen zur Aufrechterhaltung und/oder Verbesserung von funktionalen Einschränkungen (im Sinne einer Funktionserhaltung) im Alter.
Damit werden zunehmend andere Krankheitsbilder von einer rehabilitativen Betrachtung erfasst. Dazu gehören neben degenerativen Erkrankung vor wiegend des Bewegungsapparates vor allem die Folgen akuter Erkrankungen durch Arteriosklerose - Herzinfarkt und Schlaganfall.

\section{Definition - medizinisch}

Die Beurteilung eines Patienten erfolgt überwiegend funktionsbezogen. Krankheits- und organspezifische Betrachtung ist sekundär. Die medizinische Dimension dominiert im Gegensatz zur kurativen Medizin die soziale und berufliche Dimension nicht!

Der Rehabilitationsprozess stellt jedoch nur einen passageren Prozess dar, dh er ist zeitlich limitiert und keine endlose Schleife. Er führt - bildlich gesprochen - aus der Kuration entweder in die (sekundär/tertiär) Prävention oder in die Palliative Medizin.

Damit kommt es einerseits zu einer organspezifischen Spezialisierung in der Medizin und andererseits zu einer zeitlichen Fragmentierung der Zuständigkei für die Organisation des Behandlungsverlaufes. Primärprävention, Akutbehandlung, Rehabilitation, Sekundärprävention, Tertiärprävention und Palliativmedizin sollen optimal aufeinander abgestimmt werden.

\section{Zusammenfassung: Medizinische Definition}

Rehabilitation unterscheidet sich von kurativer Medizin durch eine funktionsbezogene Sichtweise.

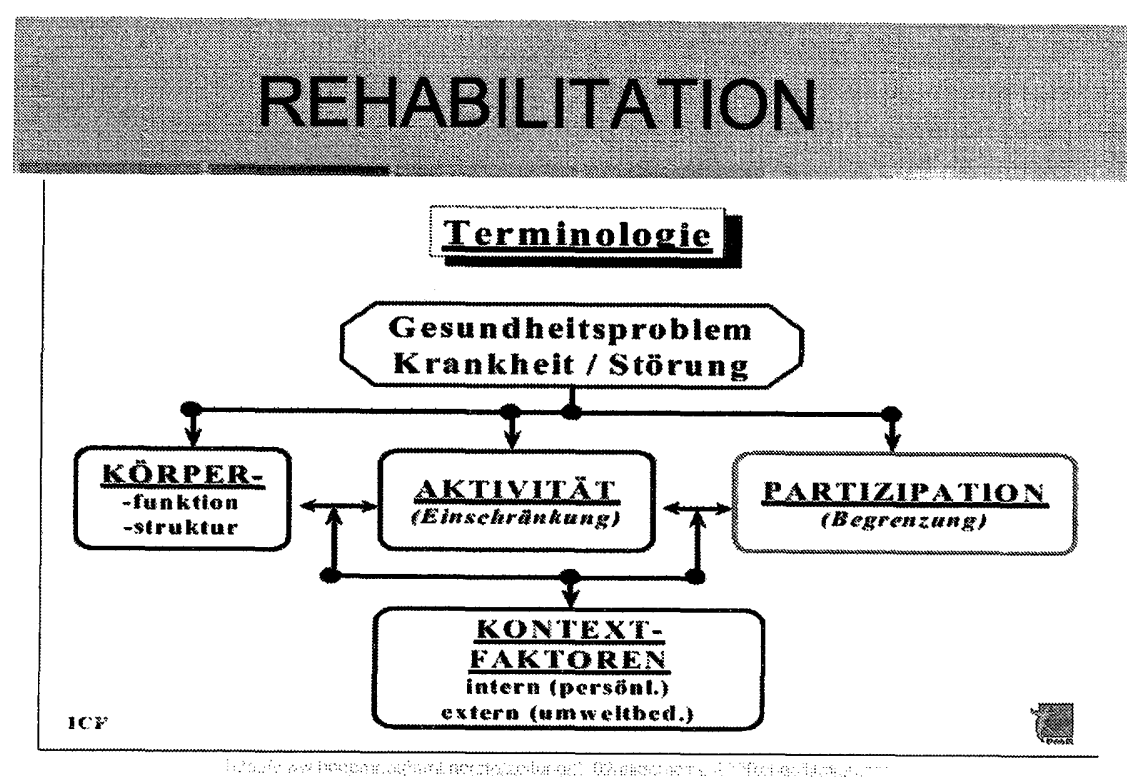

Dies drückt sich auch dadurch aus, dass zur Beschreibung der Patientencharakteristik nicht die International Classification of Diseases - ICD - sondern die 
International Classification of Functioning, Disability and Health - ICF - verwendet wird. ${ }^{1}$ )

Die verschiedenen Phasen des Behandlungsverlaufes und das Tätigwerden verschiedener Dienstleister müssen während der Phase der Rehabilitation aufeinander abgestimmt werden. Dazu ist auch eine Wertung und Entscheidung sowie eine Mitwirkung durch den Rehabilitanden erforderlich, um seinen Präferenzen für die Lebensgestaltung entsprechen zu können.

\subsection{Rechtliche Definition}

Auf diesen Bereich soll hier nur aus der Sicht eines Mediziners eingegangen werden. Eine vertiefte Darstellung ist den juristischen Beiträgen vorbehalten.

Definition - rechtlich (ASVG):

„\$116. (1) Die Krankenversicherung trifft Vorsorge

1. für die Früherkennung von Krankheiten und die Erhaltung der Volksgesundheit;

2. für die Versicherungsfälle der Krankheit, der Arbeitsunfähigkeit infolge Krankheit und der Mutterschaft:

3. für Zahnbehandlung und Zahnersatz sowie für die Hilfe bei körper-

lichen Gebrechen;

4. für medizinische Maßnahmen der Rehabilitation;

5. für die Gesundheitsförderung.

(2) Überdies können aus Mitteln der Krankenversicherung

1. Maßnahmen zur Festigung der Gesundheit $(\$ 155)$ und

2. Maßnahmen zur Krankheitsverhütung $(\$ 156)$...

Diese Darstellung des \$116 ASVG zeigt, dass die KV nur für den Bereich der medizinischen Maßnahmen der Rehabilitation zuständig ist. Diese zeitlich jüngste Regelung für Rehabilitation im österreichischen Recht wurde allein aus dem Motiv eingeführt, diese Behandlungsmöglichkeiten auch Personen ohne eigenen Leistungsanspruch aus der Pensionsversicherung zugänglich zu machen. Bis zur 50. ASVG Novelle waren derartige Leistungen „mitversicherten Angehörigen" nicht zugänglich.

Der Inhalt der medizinischen Maßnahmen der Rehabilitation und ihr Zusammenhang mit der Krankenbehandlung, welche als „Vorsorge für die Versicherungsfälle der Krankheit“" zu verstehen ist, wird in \$154a geregelt. Dort findet sich auch ein Verweis auf $\$ 133$ Abs 2 und damit eine qualitative und ökonomische Beschreibung des Umfanges der Leistungen.

Der Text zeigt auch, dass die Zielsetzungen der (kurativen) Krankenbehandlung und der Rehabilitation weitgehend deckungsgleich sind. Diese „Kernbestimmung" des österreichischen KV-Rechtes lautet:

\section{$\$ 133$ Abs 2}

„Die Krankenbehandlung muß ausreichend und zweckmäßig sein, sie darf jedoch das Maß des Notwendigen nicht überschreiten. Durch die Krankenbe-

1) Allgemeine Rehabilitation: Grundlagen und Prinzipien; Bochdansky/Prager/ Ammer, ÖZPMR 2002, 12/2. handlung sollen die Gesundheit, die Arbeitsfähigkeit und die Fähigkeit, für die lebenswichtigen persönlichen Bedürfnisse zu sorgen, nach Möglichkeit wiederhergestellt, gefestigt oder gebessert werden. Die Leistungen der Krankenbehand lung werden, soweit in diesem Bundesgesetz nichts anderes bestimmt wird, als Sachleistungen erbracht."

Definition - rechtlich (ASVG):

„\$154a. (1) Die Krankenversicherungsträger gewähren, um den Erfolg der Krankenbehandlung zu sichern oder die Folgen der Krankheit zu erleichtern, im Anschluß an die Krankenbehandlung nach pflichtgemäßem Ermessen und nach Maßgabe des $\$ 133$ Abs. 2 medizinische Maßnahmen der Rehabilitation mit dem Ziel, den Gesundheitszustand der Versicherten und ihrer Angehörigen so weit wiederherzustellen, daß sie in der Lage sind, in der Gemeinschaft einen ihnen angemessenen Platz möglichst dauernd und ohne Betreuung und Hilfe einzunehmen."

Betrachtet man diese beiden Regelungsbereiche gemeinsam, so ist der wesentliche Unterschied die zeitliche Abfolge. Für den Beginn der Rehabilitation soll die Krankenbehandlung (zumindest in Teilen) „abgeschlossen“ sein. Es is allerdings leicht einsichtig, dass eine sekundäre oder tertiäre Prävention zeitgleich erfolgen muss. Die medizinischen Inhalte sind zumindest teilweise identisch und sowohl aus kurativer als auch aus rehabilitativer Sicht indiziert.

Ein weiterer rechtlicher Unterschied besteht in der Form der Gewährung der Leistungen. Dies hat vor allem auf die Organisation des Gesundheits- und Sozialsystems Auswirkungen, welche noch später gesondert dargestellt werden müssen. Hier soll nur die Notwendigkeit einer Antragstellung und die faktisch eingeschränkte Überprüfbarkeit der Erledigung als rechtliches Unterscheidungskriterium genannt werden.

Für Versicherte kann auch die unterschiedliche Regelung von Kostenanteilen - „Selbstbehalten“ - von Bedeutung sein. Eine genaue Darstellung erforder allerdings ein Eingehen in die einzelnen SV-Gesetze und wird hier daher unterlassen.

Im Gegensatz zur Krankenbehandlung ist die Leistung Rehabilitation nicht nur in den Bestimmungen der KV-Träger sondern auch im Recht der PV und de UV geregelt. Die historische Wurzel findet sich - wie bereits oben dargestellt - ja in der Unfallheilbehandlung.

Definition - rechtlich:

$\$ 300$. (1) Die Pensionsversicherungsträger treffen Vorsorge für die Rehabilitation von Versicherten und Beziehern einer Pension aus einem Versicherungsfall der geminderten Arbeitsfähigkeit, ausgenommen eine Knappschaftspension die an einer körperlichen, geistigen oder psychischen Behinderung leiden.

(2) Versicherte gelten als behindert im Sinne des Abs. 1, wenn sie infolge eines Leidens oder Gebrechens ohne Gewährung von Maßnahmen der Rehabilitation die besonderen Voraussetzungen für eine Pension aus dem Versicherungsfall der geminderten Arbeitsfähigkeit, ausgenommen eine Knappschaftspension, wahrscheinlich erfüllen oder in absehbarer Zeit erfüllen werden; vorwiegend 
altersbedingte Leiden und Gebrechen gelten nicht als Leiden und Gebrechen im Sinne dieses Absatzes.

(3) Die Rehabilitation umfasst medizinische und berufliche Maßnahmen und soweit dies zu ihrer Ergänzung erforderlich ist, soziale Maßnahmen mit dem Ziel, Behinderte bis zu einem solchen Grad ihrer Leistungsfähigkeit herzudem Ziel, Behinderte bis zu einem solchen Grad ihrer Leistungsfahigkeit herzuwirtschaftlichen Leben und in der Gemeinschaft einen ihnen angemessenen Platz möglichst dauernd einnehmen zu können."

Der Leistungsumfang geht dabei über die medizinischen Maßnahmen hinaus und umfasst auch berufliche und soziale Interventionen. Ob der Leistungsumfang der Rehabilitation durch die PV ebenfalls der qualitativen und ökonomischen Einschränkung des $\$ 133$ Abs 2 ASVG folgt, wird unterschiedlich interpretiert. Der Leistungsumfang der UV geht darüber unstrittig hinaus.

\section{Zusammenfassung: Rechtliche Definition}

Für Versicherte ergeben sich unterschiedliche leistungsrechtliche Rahmenbedingungen für die Inanspruchnahme von medizinischen Leistungen abhängig davon, welcher Rechtstitel bei welchem Versicherungsträger (in welcher Versicherungssparte) als (Antrags-)Inhalt gesehen wird. Idente Leistungen können als Krankenbehandlung und dort wiederum als sekundäre oder tertiäre Prävention, als Rehabilitation aus der KV oder der PV und als Unfallheilbehandlung und Rehabilitation aus der UV angesehen werden. Die jeweiligen Anspruchsvoraussetzungen, Subsidiaritätsregelungen und Unterschied im Leistungsumfang sind für Patienten und auch Gesundheitsdienstleister intransparent und unklar. Dadurch entsteht häufig der Eindruck der Willkür bei der Ausübung des pflichtgemäßen Ermessens. Auch der unterschiedliche Umfang des Leistungsanspruches ist für die Betroffenen nicht verständlich. Es darf bezweifelt werden, dass die Komplexität und Ungleichheit der Regelungen dem Normgeber bewusst ist und er damit gezielt eine Differenzierung der Leistungsansprüche beabsichtigt.

\subsection{Organisatorische Definition}

Die zuvor dargestellten rechtlichen Unterschiede prägen auch die organisatorische Ausgestaltung des Sozialsystems. Dabei sind insbesondere die $\mathrm{Zu}-$ gangsregelungen zu Leistungen, die Art der Leistungen und die Zuordnung des Organisationsaufwandes (bzw der sachlich inhaltlichen Entscheidungen) von Bedeutung.

\section{Zugangsregelungen}

Definition - organisatorisch:

„201a. Die Einleitung von Maßnahmen der Rehabilitation des Unfallversicherungsträgers bedarf der Zustimmung des Versehrten. Vor dessen Entscheidung ist der Versehrte vom Versicherungsträger über das Ziel und die Möglichkeiten der Rehabilitation nachweislich in geeigneter Weise zu informieren und zu beraten. Der Versehrte hat bei der Durchführung der Maßnahmen der Rehabilitation entsprechend mitzuwirken."
In der UV wird der Versicherungsträger nicht nur auf Antrag sondern auch von Amts wegen tätig. Damit ist ein Verunfallter nicht auch noch dadurch belastet, dass er selbst die Organisation seiner Behandlung und Rehabilitation übernehmen muss. Im Sinne der Schadensbegrenzung aus der Sicht des Haftenden wird alles Mögliche unternommen den Geschädigten bei der Wiederherstellung zu unterstützen. Dies setzt voraus, dass der Eintritt eines Leistungsfalles erkann werden kann. Dafür ist eine entsprechende Meldepflicht eingerichtet.

In der PV ist ein eigenständiges Erkennen eines Handlungsbedarfes aufgrund der Rahmenbedingungen nicht möglich. Daher gilt in dieser Versicherungssparte überwiegend das Antragsprinzip. Die einzige Ausnahme stellt die Früherfassung von Rehabilitationsbedarf bei langer Krankenstandsdauer dar. Dabei wird ein möglicher Rehabilitationsbedarf durch den Träger der KV aufgrund der Verlaufsdaten festgestellt. Diese organisatorischen Regelungen stammen aus einer Zeit der papiergebundenen Administration und händischen Sachbearbeitung. Die Möglichkeiten des Einsatzes der elektronischen Datenverarbeitung haben zu verkürzten Meldefristen in allen Bereichen der SV und des Gesundheitssystems geführt. Die rechtlichen Regelungen zur Organisation der Zusammenarbeit der unterschiedlichen SV-Träger im Bereich der Rehabilitation wurden allerdings an diese neuen Möglichkeiten bisher nur unzureichend angepasst. Damit bleibt organisatorisch der Zugang zur Rehabilitation in der PV weiterhin maßgeblich an das Antragsprinzip gebunden.

Definition - organisatorisch:

„\$361. (1) Die Leistungsansprüche sind von den Versicherungsträgern im Rahmen ihrer örtlichen und sachlichen Zuständigkeit festzustellen

1. in der Kranken- und in der Pensionsversicherung auf Antrag,

2. in der Unfallversicherung von Amts wegen oder, sofern das Verfahren nicht auf diese Weise eingeleitet wurde, auf Antrag.

Ein Antrag auf eine Pension aus den Versicherungsfällen der geminderten Arbeitsfähigkeit gilt auch als Antrag auf Leistungen der Rehabilitation."

In der KV wäre ein Rehabilitationsbedarf grundsätzlich erkennbar, aber die Zugangsregelung sieht auch hier eine Antragstellung vor. Eine mögliche patientenorientierte Gestaltung der Organisation könnte hier vorsehen, dass die Inanspruchnahme taxativ bestimmter Leistungen der Krankenbehandlung einer Antragstellung auf Rehabilitation - unabhängig von einer allfälligen Zuständigkeit - gleichgehalten werden. Eine vergleichbare Regelung sieht vor, dass Anträge auf eine Pension wegen geminderter Erwerbsfähigkeit als Anträge auf Rehabilitation gelten. Damit wird ein akut Erkrankter von der Aufgabe entlastet, seinen weiteren Behandlungsverlauf selbst zu koordinieren. Auch ist er bei einer solchen Regelung nicht darauf angewiesen, dass die Gesundheitsdienstleister in der Akutsituation nicht nur optimal ihrer organspezifischen Sichtweise folgen sondern auch eine langfristige funktionelle und soziale Perspektive, welche in der Frühphase meist nicht einmal annähernd abschätzbar ist, beachten.

Damit könnte iS sozialer Rechtsgestaltung ein erleichterter Zugang zu Rehabilitationsleistung erreicht werden, wenn auch damit das Antragsprinzip aufrechterhalten und die Ausübung des pflichtgemäßen Ermessens nicht von Amts wegen aufgetragen wird. 
$\$ 305$ ASVG regelt das Vorgehen zwischen Versichertem und Versicherung. Dabei ist erkenntlich, dass für die geforderte Information und Beratung jedenfalls eine ausreichende Tatsachenfeststellung (iS einer Begutachtung) erforderlich ist. Die derzeitige Praxis sieht dabei vor, dass ein medizinisches Sachverständigengutachten für die Antragsbeurteilung eingeholt wird. Ob dabei eine physische Untersuchung oder nur ein Aktengutachten erforderlich ist, wird im Einzelfall entschieden. Auch für die Administration dieses Prozessschrittes kann durch eine technische Unterstützung eine Beschleunigung und Verbesserung erwartet werden.

\section{Definition-organisatorisch:}

$\$$ 305. Der Behinderte ist vom Versicherungsträger über das Ziel und die Möglichkeiten der Rehabilitation nachweislich in geeigneter Weise zu informieren und zu beraten. Der Behinderte hat bei der Durchführung der Maßnahmen der Rehabilitation entsprechend mitzuwirken."

Wie schon aus der Existenz des Antragsprinzipes geschlossen werden kann, wird die Erbringung von Rehabilitationsleistungen nicht als zeitkritisch angesehen. Es wird für eine Durchführung „im Anschluss an die Krankenbehandlung“ ein für Antrag, Begutachtung und Information ausreichender Zeitraum angenommen.

\section{Art der Leistungen}

Die Art der Leistungen in der Rehabilitation unterscheidet sich in zweierlei Hinsicht: In ihrem Inhalt - medizinische, berufliche und soziale Leistungen und in ihrer Organisation - Sachleistungen und Vergütung / Kostenerstattung.

Der Inhalt wird einerseits von den Bedürfnissen im Einzelfall aber andererseits auch von den Möglichkeiten des zuständigen Leistungsträgers bestimmt. Berufliche und soziale Maßnahmen werden durch KV-Träger nicht erbracht. Eine "gemischte Zuständigkeit" ist nicht vorgesehen. Die Organisation der Leistungserbringung für Leistungen der Krankenbehandlung als flächendeckendes Sachleistungssystem mit der Möglichkeit der Inanspruchnahme ohne vorherige Antragstellung resultiert aus der Notwendigkeit für Akuterkrankung „rund um die Uhr" Vorsorgen zu müssen. Dies wäre grundsätzlich auch durch ein System der Kostenerstattung möglich, jedoch bedeutet die dort gegebene Notwendigkeit Leistungen vorzufinanzieren ein beträchtliches Zugangshemmnis. Da ein rascher und „niederschwelliger" Zugang zu Leistungen der Krankenbehandlung ein wesentliches Qualitätsmerkmal von Gesundheitssystemen darstellt, wurde diese Systemgestaltung zu Recht für das österreichische KV-System ausgewählt. Die Kostenerstattung bleibt daher in der Regel Leistungsbereichen vorbehalten, welche einer allgemeinen Regelung wegen fehlender Häufigkeit, fraglicher Zweckmäßigkeit und unklarer Kosteneffektivität nicht zugänglich sind. Die Ausgestaltung über Gesamtverträge und davon abhängige Einzelverträge erlaubt es den Leistungsumfang und die Tarife auf der Ebene der Interessensvertretungen zu verhandeln und festzulegen.

\section{REHABILITATION}

DEFINITION - Rechtlich

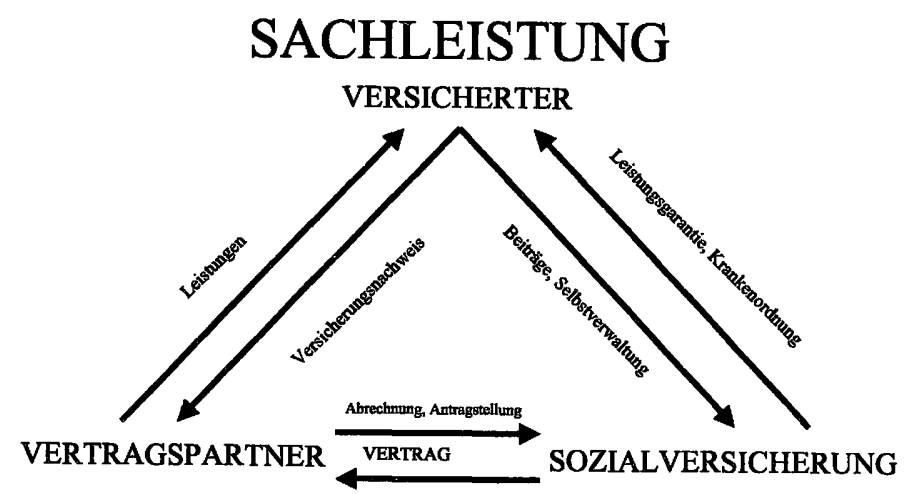

Alle Versicherungsträger haben die Erbringung von medizinischen Leistungen der Rehabilitation in Krankenanstalten, welche überwiegend der Rehabilitation dienen, selbst vertraglich geregelt. Schrittmacher für diesen Leistungsbereich waren die Träger der PV. Die spezialisierte Leistungserbringung in eigenen Sonderkrankenanstalten stammt aus der erweiterten Unfallheilbehandlung und wurde durch die PV-Träger auf andere medizinische Indikationsbereiche ausgedehnt Aber nur die KV-Träger verfügen über eine Infrastruktur von Vertragspartnern zur Erbringung von medizinischen Leistungen als Sachleistungen außerhalb von Krankenanstalten. Werden solche Maßnahmen im Laufe eines komplexen Rehabilitationsplanes benötigt, so besteht die Möglichkeit, dass Träger der UV und der PV die Leistungsinfrastruktur der KV nutzen und diese Leistungen nachträglich an sich ziehen. Dies gilt auch für die Organisation der Medikamentenversorgung und den Zugang zu Leistungen von Gesundheitsdienstleistern, welche der ärztlichen Hilfe gleichgestellt sind.

Diese zweckmäßige Kombination der Leistungsinfrastruktur könnte im Sinne einer Verbesserung für die Versicherten auch in einer gemeinsamen $\mathrm{Zu}$ ständigkeit münden. Als Vorteil für die Patienten käme damit die Beratungskapazität der PV und UV bezüglich beruflicher und sozialer Maßnahmen, welche derzeit von den KV-Trägern nicht erbracht werden dürfen, auch Rehabilitanden der KV zugute. Umgekehrt ist der Erfolg einer Rehabilitation eines Krankheitsgeschehens oft von einer umfassenden Krankenbehandlung in anderen Bereichen abhängig. Die Feststellung der „Rehabilitationsfähigkeit“ wird allerdings dadurch beeinträchtigt, dass diese Bereiche nicht ausreichend erkannt, beobachtet und eventuell auch beeinflusst werden können. 


\section{REHABILITATION}

\section{ABGRENZUNGSPROBLEME}

\section{KRANKENBE- \\ HANDLUNG}

- Wird als Sachleistung

bereitgestellt

erbracht

Wird nach subjektive

Empfinden in Anspruch

genommen

Ist nicht verbindlich aber

einklagbar
* Wird unkoordiniert
REHABILITATION

KUR

\author{
management bei der \\ Behandlung \\ Ist eine Teamleistung des \\ Rehabilitanden und der SV \\ * Ist für die Beteiligten in \\ der Durchführung \\ verbindlich
}

* Begleitende Therapie mit einem lokalen Heilmittel Life-style Medizin - Freiwillige Leistung zur Gesundheitsvorsorge * Gesundheits-erziehung und

Die hier dargestellten Gesichtspunkte zeigen auch, dass eine Betrachtung der „Leistung an sich“ keinen Aufschluss darüber gibt, ob sie mit der Intention der Krankenbehandlung, der Rehabilitation, der Palliation oder der Prävention eingesetzt wird. Für die Betroffenen ist die Unterscheidung daher in der Regel unverständlich. Eine Abgrenzung anhand der organisatorischen Kennzeichen lässt die Rehabilitation gegenüber der Krankenbehandlung als bürokratisch er- . scheinen.

Weitere organisatorische Unterscheidungen finden sich auch in der Rollenbeschreibung der einzelnen Akteure. In der Krankenbehandlung fragt der Patient aufgrund seiner subjektiv beeinträchtigten Gesundheit in der Regel Gesundheitsdienstleistungen bei einem Vertragspartner seiner Versicherung nach. Theoretisch wird sowohl das Verhalten des Patienten durch die Vorgaben des Leistungsrechts als auch das Verhalten des Leistungserbringers durch seinen Einzelvertrag (mit-)bestimmt. Die Entscheidungsautonomie liegt (fast) ausschließlich beim Patienten, wird allerdings aus empirischer Sicht überwiegend durch den Informationsvorsprung des Gesundheitsexperten gesteuert. Dies ist der Mechanismus der "angebotsinduzierten Nachfrage“. Die Rahmenbedingungen werden damit durch Leistungs- und Vertragsrecht, die Detailentscheidungen durch Patient und Gesundheitsdienstleister festgelegt. Auf die unterschiedliche Gestaltung der ArztPatientenbeziehung - paternalistisch, partizipativ oder demokratisch - wird hier nicht näher eingegangen.

In der Rehabilitation wird das Vorgehen zwischen dem Versicherten als Patienten und seiner Versicherung als (indirektem) Leistungserbringer (un)mittelbar festgelegt. Deshalb benötigt die Versicherung auch eine medizinische Tatsachenfeststellung und Empfehlung über das weitere Vorgehen. Nur so kann der Rehabilitationsplan gemeinsam erstellt und beschlossen werden.
Definition - organisatorisch:

- nach Feststellung des Rehabilitationsbedarfes,

- gemeinsamer Festlegung eines Rehabilitationszieles und

- gemeinsamer Festlegung eines Rehabilitationsplanes.

Die Durchführung wird entweder über eigene Einrichtungen unmittelbar oder mittelbar durch Vertragspartner erbracht. Für alle Beteiligten entsteht durch den Rehabilitationsplan eine deutlich höhere Verbindlichkeit als im Setting der Krankenbehandlung. So kann ein nicht ausreichend begründetes Abweichen von der vorgesehenen Behandlung durch den Versicherten zur Versagung anderer Leistungen - wie dem Übergangsgeld - führen.

Der Begriff der Rehabilitation hat in organisatorischer Hinsicht vielfältige Aspekte, welche so wie die rechtlichen Rahmenbedingungen den Betroffenen meist nicht bewusst sind. Auch besteht in diesem Bereich noch beträchtlicher Spielraum für eine Verbesserung im Sinne einer Ausrichtung an den Bedürfnissen der Patienten.

\section{Zusammenfassung: Organisatorische Definition}

Wesentliche Bedeutung haben die unterschiedlichen Zugangsregelungen zu Leistungen. Im Krankheitsfall ist das Interesse eines Patienten auf eine rasche $\mathrm{Ge}$ sundung gerichtet und die Notwendigkeit sich während einer Phase ausgeprägter Leistungseinschränkungen mit komplexen Regelungen und Planungen auseinanderzusetzen stellt eine unzumutbare Überforderung dar. Während einer akuten Erkrankung sinkt die persönliche Entscheidungskompetenz auf das Niveau eines Kindes. Es sind daher von Seiten des Gesundheits- und Sozialsystems Vorkehrungen zu treffen, dass eine Entlastung von vorhersehbaren Entscheidungen besteht und für andere Fragen eine kompetente Unterstützung aktiv angeboten wird. Nur dadurch kann die Zielsetzung der Krankenbehandlung und Rehabilitation erreicht werden.

Definition-organisatorisch:

Rehabilitation :

- ist Information/ Beratung/ Organisation,

- ist Schnittstellenmanagement bei der Behandlung,

- ist „Hilfe zur Selbsthilfe“,

- ist eine Teamleistung des Rehabilitanden und der SV,

- erfordert ein Feststellungsverfahren.

Ob dafür als Infrastruktur Case Manager, Rehabilitationsberater, Sozialarbeiter oder andere Mitarbeiter von Sozialversicherungen und Betreuungsinstitutionen eingesetzt werden, wird in Abhängigkeit des regionalen Settings differieren. Es ist allerdings eine gleichartige Vorgangsweise sicherzustellen:

Definition - organisatorisch:

- nach Feststellung des Rehabilitationsbedarfes,

- gemeinsamer Festlegung eines Rehabilitationszieles und

- gemeinsamer Festlegung eines Rehabilitationsplanes. 
Für die Zukunft sollten die Rahmenbedingungen patientenzentriert ausgerichtet werden. Die derzeitigen Regelungen bedienen vor allem die Interessen existierender Organisationen und die Bestandssicherung und Ab-(Aus-)grenzungswünsche von Leistungsanbietern. Insbesondere ist eine Zusammenarbei der doch unterschiedlich spezialisierten Institutionen vorzusehen, da eine ungeteilte Zuständigkeit zwar historisch raschere Entscheidungen ermöglichte, aber heute Organisationsmöglichkeiten bestehen, welche eine bessere patientenzentrierte Koordination unterschiedlicher Leistungsbereiche und Spezialisierungen ermöglichen.

\section{Beispiel: Myocardinfarkt}

Am Beispiel des Herzinfarktes sollen die dargestellten theoretischen Überlegungen nunmehr exemplarisch diskutiert werden.

\subsection{Zeitablauf}

Der Krankheitsverlauf eines Herzinfarktes zeigt zwar im Detail eine be trächtliche Variationsmöglichkeit, kann aber idealtypisch dargestellt werden Daher konnten auch für die Rehabilitation Leitlinien erstellt werden. ${ }^{2}$ )

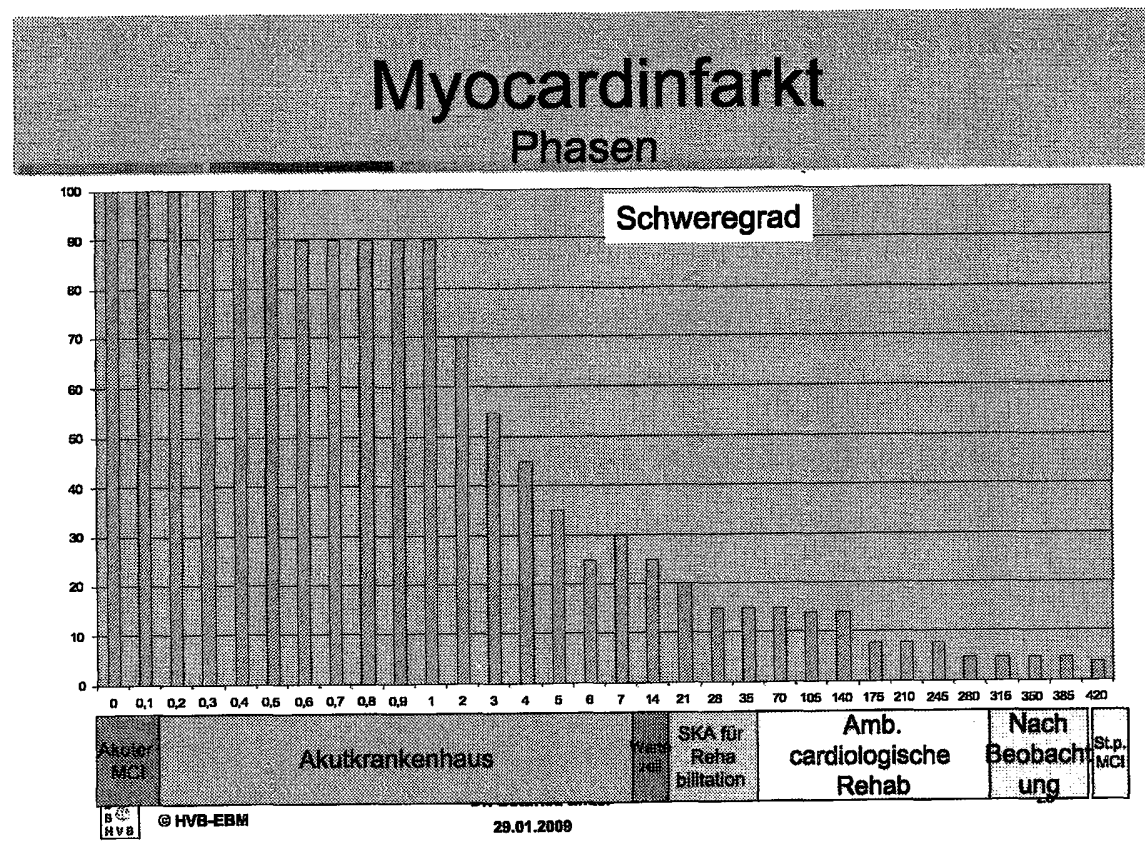

Häufig tritt die Erkrankung ohne wesentliche Vorzeichen plötzlich auf Druck und Brennen im Brustbereich, Vernichtungsgefühl, Angst, Atemnot of mit Ausstrahlung in den linken Arm sind klassische Symptome eines Herzinfarktes. Ein als Notfall anzusehendes, schweres, lebensbedrohliches und nich selten auch sofort tödliches Krankheitsgeschehen nimmt damit seinen Anfang Vom Patienten oder Helfern ist in diesem Falle sofort über den Ärztenotruf ein Notarzteinsatz anzufordern. Der Notarzttransport erfolgt so rasch als möglich und nach allfällig vorher notwendiger Behandlung in ein geeignetes Krankenhaus - optimal, wenn in diesem Krankenhaus die Möglichkeit einer Revaskularisation (das heißt meist einer Aufdehnung eines verschlossenen Blutgefaßßes) gegeben ist und dies auch in der dafür erforderlichen Zeitspanne erfolgen kann. Gelingt diese Akutversorgung optimal, so gehen auch der Schweregrad der Erkrankung und die damit verbundene Gefährdung zurück.

In der Akutkrankenhausbehandlung stabilisiert sich die Belastbarkeit des patienten und nach erfolgreicher Revaskularisation ist meist kein langer Krankenhausaufenthalt nötig. Die Leistungsfähigkeit und Belastbarkeit sind als funktionelle Aspekte allerdings deutlich reduziert. Daher soll jedenfalls eine Rehabilitation durchgeführt werden. Gleichzeitig wird auch stationär bereits mit der sekundären Prävention zur Vermeidung eines weiteren Infarktes begonnen.

Die Rehabilitation wird zeitlich in vier Phasen eingeteilt. Dabei erfolgt die Durchführung der Phase 1 bereits in der Akutkrankenanstalt. Vor der Aufnahme in eine Sonderkrankenanstalt zur Durchführung der Phase 2 entsteht meist aus organisatorischen Gründen eine Wartezeit. Diese wird von Patienten als sehr belastend empfunden, da die noch reduzierte Belastbarkeit unter Alltagsbedingungen als stärkere Einschränkung empfunden wird, als im geschützten Umfeld einer Krankenanstalt. Insbesondere bei bereits vorbestehenden Leistungsminderungen oder fehlender sozialer Unterstützung kann sich in dieser Zeit der Gesundheitszustand wieder verschlechtern.

Nach der Phase 2, welche in der Regel drei Wochen benötigt, kann eine Phase 3 über sechs bis neun Monate angeschlossen werden. Diese dient dazu, in der Phase 2 begonnene Verhaltensänderungen und Steigerungen der Leistungsfähigkeit weiter zu verbessern oder zumindest zu erhalten. Die Phase 4 unterscheidet sich nicht wesentlich von den Empfehlungen für eine gesunde und aktive Lebensführung, wie sie auch in der Primär- und Sekundärprävention empfohlen wird.

$\left.{ }^{2}\right)$ http://www.agakar.at/aerzte/guidelines $1 . h t m$ 

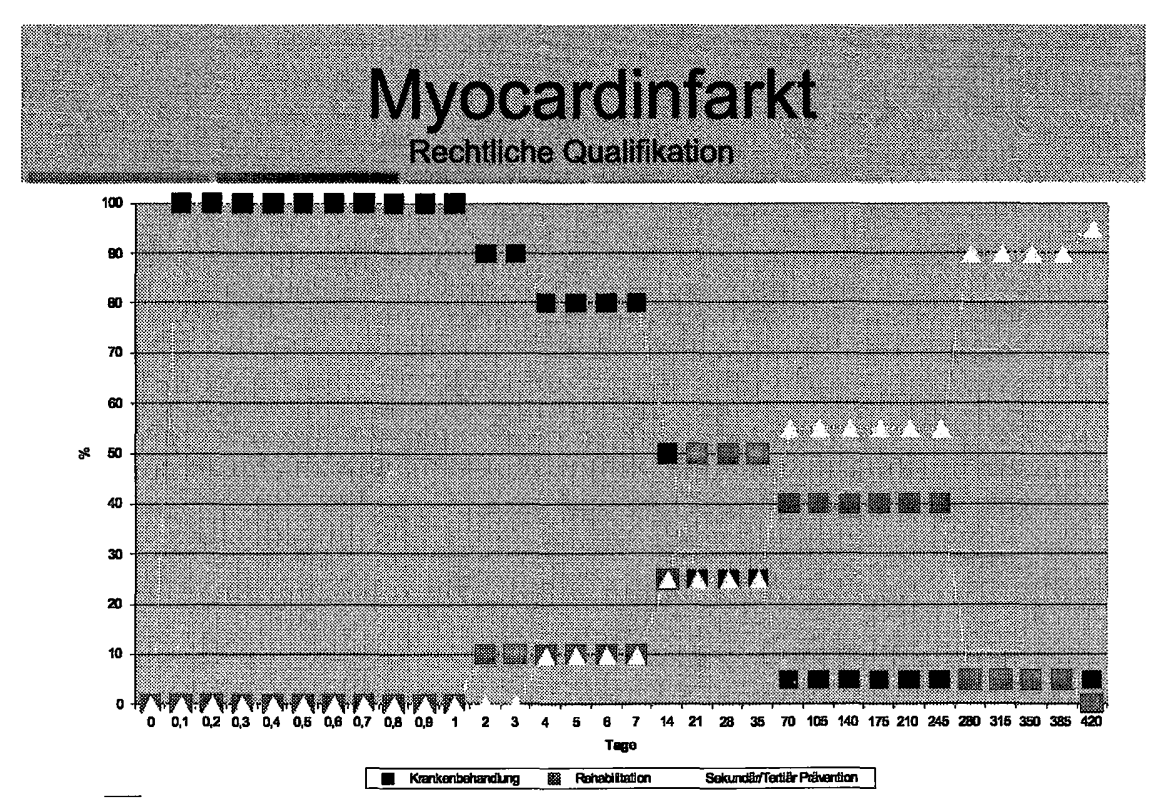

Aus leistungsrechtlicher Sicht dominiert in der Akutphase die KV-Zuständigkeit. Rehabilitation der Phase 1 und Beginn der Sekundärprävention werden in der Regel nicht getrennt ausgewiesen. Während der Phase 2 findet einerseits Rehabilitation und andererseits Krankenbehandlung sowohl im kurativen als auch im sekundär präventiven Sinne statt. Hier wird, obwohl sie einen beträchtlichen Anteil am Geschehen innehat, während des Aufenthaltes in einer Sonderkrankenanstalt die Krankenbehandlung nicht getrennt dargestellt.

In der Rehabilitationsphase 3 können als Rehabilitation ausgewiesene Sachleistungen eingesetzt werden, aber die Verhaltensänderungen und das erforderliche körperliche Training werden teilweise auch von den Betroffenen aus eigenem Antrieb selbst durchgeführt. Bei einer Entscheidung des Patienten gegen die empfohlene Vorgangsweise kann auch die Verfügbarkeit von Sachleistungen die Inanspruchnahme nicht verbessern. Dies ist auch daran ersichtlich, dass die erforderliche Krankenbehandlung und Sekundärprävention in vielen Fällen abgebrochen wird. Ist das Ziel einer funktionellen Wiederherstellung erreicht worden, so bleiben nach dem derzeitigen Stand der Wissenschaft eine lebenslange Notwendigkeit einer Sekundärprävention und möglicherweise ein Krankenbehandlungsbedarf einer meist vorliegenden Grundkrankheit. Die Lebensführung entsprechend der Phase 4 ist, wie bereits dargestellt, eigentlich krankheitsunabhängig empfehlenswert.

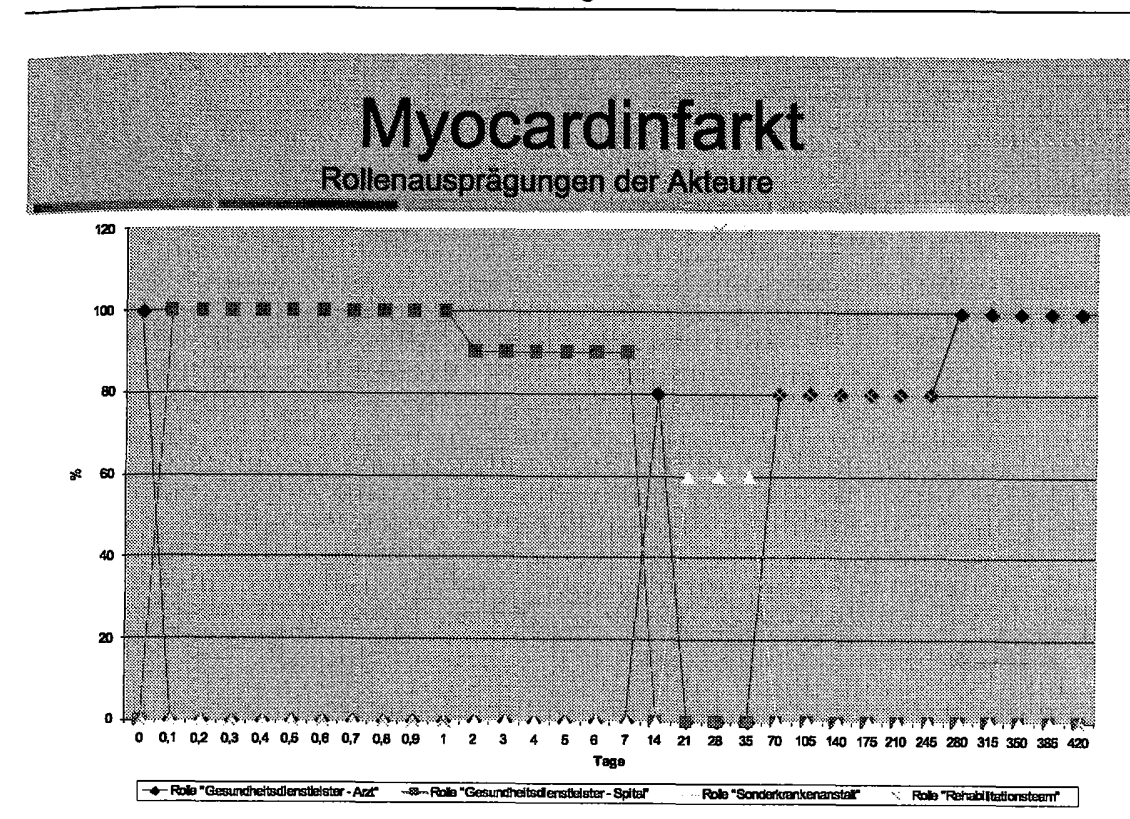

Obige Graphik soll die Beteiligung der unterschiedlichen Dienstleister im Zeitablauf darstellen. Ersichtlich ist vor allem, dass die stationären Einrichtungen aber auch das Rehabilitationsteam zeitlich klar beschränkte Zeitabschnitte in unterschiedlicher Intensität betreuen. Dies liegt unter anderem auch daran, dass bei inhaltlich identen Maßnahmen diese in den eigenen Wirkungsbereich gezogen und dort erbracht werden.

Auch der Beitrag der Sozialversicherung zum Geschehen zeigt eine derartige zeitliche Schwankung. Die Aufgabe als Versicherung bleibt durchgehend bestehen. Für die Zeit der intensiven Betreuung im Sinne eines Case Managements und während der Erbringung von Rehabilitationsleistungen durch eigene Einrichtungen und die Mitwirkung im Rehabilitationsteam nimmt die Bedeutung dieser Tätigkeiten relativ gesehen etwas ab. Nach Abschluss der Rehabilitation und der Evaluation des Ergebnisses tritt wieder der ursprüngliche Zustand ein. 

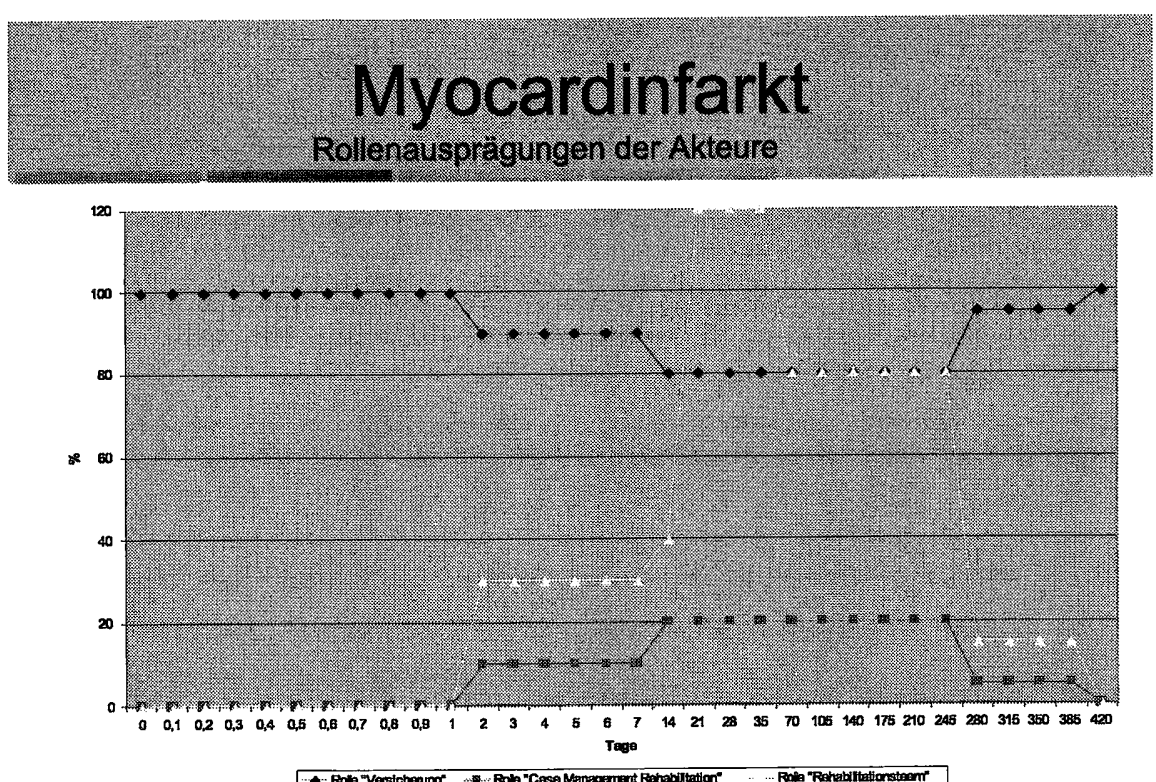

In ähnlicher Form verändert sich auch die Rolle des Versicherten während der Rehabilitation. Ist er vor der akuten Erkrankung gegenüber dem KV-System "Konsument" und unter Berücksichtigung der dargestellten Rahmenbedingungen autonom, so verliert er wären des vereinbarten Rehabilitationsablaufes mit seiner Verbindlichkeit und allfälligen rechtlichen Folgen doch einen Teil dieser Autonomie.
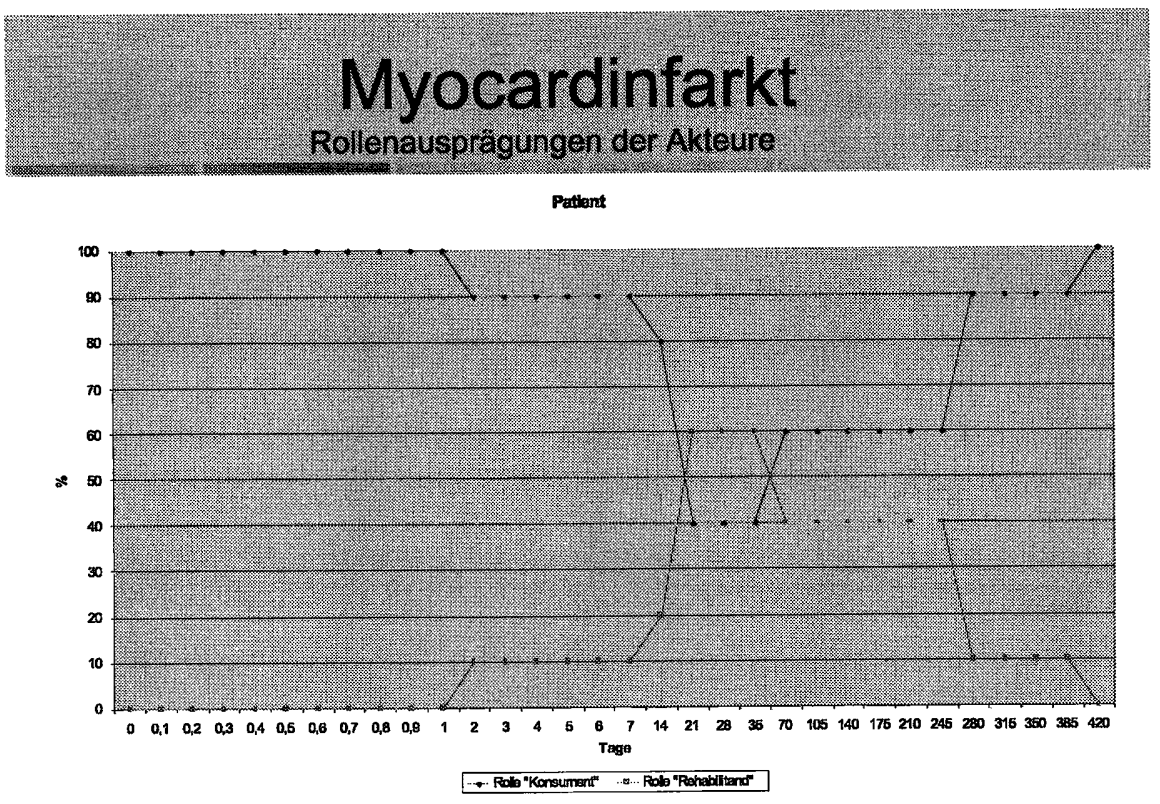

\subsection{Zusammenfassung: Beispiel Myocardinfarkt}

Als Beispiel wurde bewusst eine Krankheit aus dem Fachbereich der Inneren Medizin gewählt, damit die universelle Anwendbarkeit des funktionsbezogenen Zuganges der Rehabilitation besser verdeutlicht werden kann. Diese lebensbedrohende Erkrankung kann bei optimalem Verlauf mit einer Restitutio ad Integrum behandelt werden. Sie geht allerdings auch mit einem idR reversiblen Verlust an Leistungsfähigkeit und Belastbarkeit einher. Es verbleibt ein dauernder Bedarf an Krankenbehandlung im Sinne der Sekundärprävention. Der Bedarf an Akutbehandlung ist zeitlich kurz und wird durch Fortschritte der medizinischen Wissenschaft zunehmend kürzer. Der Bedarf an Unterstützung im sozialen und eventuell auch beruflichen Umfeld, vor allem aber für die Organisation der notwendigen Behandlung und Anleitung zur Lebensstiländerung nimmt hingegen laufend zu. Die Verläufe von Krankheitsintensität, Lebensbedrohung, Leistungserbringung verschiedener Dienstleister und Rollenausprägungen wechseln im zeitlichen Verlauf mit vielen Überschneidungen. Als Folge der Spezialisierung in der rechtlichen Ausgestaltung unseres Sozialsystems und der Tätigkeiten der Gesundheitsdienstleister nimmt der Koordinationsbedarf ebenfalls stark zu. Wird diese Organisationsaufgabe nicht ausreichend wahrgenommen, so können die durch die einzelnen Maßnahmen möglichen Vorteile für die Patienten nicht erreicht werden.

\section{Zusammenfassung}

Rehabilitation stellt eine medizinische Vorgangsweise dar, welche einer funktionellen Betrachtung die Priorität gegenüber der organpathologischen Betrachtung einräumt. Damit ist sie theoretisch fächerübergreifend einsetzbar. Praktisch benötigen Patienten jedoch immer mehrere dieser analytisch - theoretisch abgegrenzten Zugänge. Rehabilitation ohne Beachtung des Krankheitsbildes ist zum Scheitern verurteilt. Kurative Medizin ohne Rehabilitation, (zumindest sekundäre) Prävention und palliativen Möglichkeiten greift ebenso zu kurz. Medizin ohne Beachtung des beruflichen und sozialen Lebensumfeldes erfüllt die Bedürfnisse von Patienten ebenfalls nicht. Die zunehmende Spezialisierung und Arbeitsteilung stellt immer höhere Anforderungen an die patientenzentrierte Organisation der Leistungserbringung.

Auch aus rechtlicher und organisatorischer Sicht stellt Rehabilitation einen eigenständigen Bereich mit massiven Abhängigkeiten von anderen Aspekten des SV-Bereiches dar. So ist die Zuständigkeit für die Erbringung von Rehabilitationsleistungen auf Träger aus allen drei Leistungssparten verteilt. Dar Leistungsumfang variiert ebenfalls abhängig von dieser Zuständigkeit. Die Möglichkeiten für eine Leistungserbringung Vorsorge zu treffen sind in den Leistungssparten ebenfalls durch die Ausrichtung an den Kernaufgaben beschränkt. Für die eigentlich gebotene Kooperation und Koordination fehlen teilweise die Voraussetzungen. Auch diese Regelungen sollten patienten-/versichertenzentriert ausgestaltet werden und sich nicht an den historischen Entwicklungen der jeweiligen Leistungssparten und ihrer Organisationsformen orientieren.

Die Komplexität der Regelungen und Organisationsstrukturen führt zu Intransparenz und gefährdet damit den erreichbaren Erfolg. Hier sind die we- 
sentlichen Verbesserungspotentiale zu sehen - Information und Klarlegung für bisher noch offene Fragen können dem Ansatz Rehabilitation als Teil einer integrierten Gesundheitsversorgung den angemessenen Platz schaffen

Information und Aufklärung für

die Versicherten und

- die GDAs

sind dringend erforderlich!

Die Umsetzung erfordert Ressourcen, dabei müssen Koordination und Verantwortlichkeit klargestellt werden.

Wissenschaftlicher Erkenntnisgewinn hat in allen Bereichen eine enorme Leistungssteigerung bewirkt. Diese Leistungssteigerung gehen allerdings teilweise durch mangelnde Koordination und Information wieder verloren. Eine weitere Zunahme von voneinander unabhängigen, unkoordinierten Leistungen und Leistungsangeboten führt zwar zu mehr Aufwand aber nicht (mehr) zu mehr Erfolg. Daher ist der Optimierung der Organisation besonderes Augenmerk zu widmen.

Da alle Leistungen im Gesundheits- und Sozialbereich letztendlich den Bürgern als Versicherten, Patienten oder in einer anderen Rolle zugute kommen sollen, so ist die Systemgestaltung auch an deren Bedürfnissen und Systemzugängen auszurichten. Organisationsspezifische, standesspezifische oder ökonomisch motivierte Gestaltungen sind jedenfalls als ungeeignet $\mathrm{zu}$ bezeichnen.

\section{Roland Benkowitsch}

\section{Prozessbeschreibung der medizinischen Rehabilitation aus deutscher Sicht}

Der Beitrag gibt zunächst einen Überblick über die Grundlagen, Abgrenzungen und Störfaktoren der medizinischen Rehabilitation in Deutschland, insbesondere in Bayern. Die wichtigsten Aspekte werden sodann anhand von praktischen Beispielen erläutert. Dies geschieht aus der Sicht eines Mediziners, der als Krankenhausarzt lange Jahre neurologische Rehabilitationsfälle aus dem Akutbereich heraus veranlasst hat, der später in einer geriatrischen Rehabilitationseinrichtung solche selbst durchgeführt und schließlich als Mitarbeiter der AOK Bayern in der Bewilligung von Rehabilitationsanträgen gearbeitet hat. Insofern erfolgt die Auswahl der Aspekte und Beispiele bewusst selektiv und erhebt keinen Anspruch auf Vollständigkeit.

\section{Abgrenzung Kuration - Rehabilitation}

Die kurative Medizin konzentriert sich im Wesentlichen auf die Ursache eine Gesundheitsstörung und legt ihren Ehrgeiz vor allem in die Heilung bzw Remission einer Erkrankung, wenigstens aber auf eine Vermeidung oder Verlangsamung einer Verschlimmerung. Am Beispiel eines gelähmten Armes setzt der kurative Mediziner insofern sein ganzes Können in die Klärung der Herkunft der Lähmung. Sind Nervenstränge, die Halswirbelsäule oder das Gehirn selbst oder vielleicht die Psyche betroffen? Mit möglichst sicherer Identifikation der pathologischen $\mathrm{Zu}$ sammenhänge, eingeleiteter Therapie gegen die Ursache und begleitenden Empfehlungen schließt der Akutmediziner seine Tätigkeit an dem Fall ab.

Für die medizinische Rehabilitation - und insb für den Patienten - beginnt hier nun oft die Arbeit erst. Den Patienten beeindruckt durchaus die fachkompetente Leistung des Akutmediziners, allerdings ist er viel mehr an der Überwindung seiner Lähmung interessiert. Die Rehabilitationsmedizin nun greift die Funktionsstörung auf, betrachtet sie auf funktioneller, psychischer und sozialer Ebene, versucht die Heilung medizinisch-therapeutisch zu unterstützen und die Störung - soweit nötig - in das Leben des Betroffenen zu integrieren. Dies kann im Falle der späteren restitutio ad integrum eine zeitliche Integration im Rahmen der Wiedereingliederung sein, es kann aber im Falle einer Defektheilung auch die lebenslange Anpassung an eine Störung bedeuten.

\section{Grundlagen „Medizinische Rehabilitation"}

Vor diesem Hintergrund kommt die medizinische Rehabilitation immer dann zum Einsatz, wenn die Mittel der kurativen Medizin nicht ausreichen, um 
Folgen von (akuter) Krankheit (wie bspw Fähigkeitsstörungen, sozialen Beeinträchtigungen, Behinderung, Erwerbsunfähigkeit, Pflegebedürftigkeit) vorzubeugen, zu beseitigen, zu bessern oder eine wesentliche Verschlechterung abzuwenden. Anders ausgedrückt ist die Rehabilitation gefragt, wenn die kurative Behandlung einschließlich Heil- und Hilfsmittelversorgung nicht ausreicht, um das individuelle Rehabilitationsziel bei gegebener Rehabilitationsbedürftigkeit, Rehabilitationsfähigkeit und positiver Rehabilitationsprognose zu erreichen.

Probleme ergeben sich hier bereits insofern, als Begrifflichkeiten wie bspw "wesentliche Verschlechterung“ oder "individuelles Rehabilitationsziel“ interpretationsbedürftig sind.

Während es dem Juristen oder Verwaltungsfachmann im Nachhinein und ohne die zeitlichen und teils informationellen Beschränkungen der Akutversorgung meist gelingt, die tatsächlichen rechtlichen Grundlagen einer Maßnahme klar aufzuzeigen, fällt es dem Akutmediziner in der Eile der Routineversorgung und unter dem Druck verschiedenster konfligierender Interessen oft nicht leicht, richtig zu entscheiden. So liegt die rein praktische Umsetzung der Rehabilitationsbeantragung im Krankenhaus meist in der Zuständigkeit von Assistenzärzten in der Weiterbildung, die mehr oder weniger kurz nach dem Studium ganz andere Prioritäten setzen als die Auslegung von Paragraphen der Sozialgesetzgebung, sofern diese überhaupt bekannt sind. Beantragung und Verlängerung einer Rehabilitationsleistung geschehen also im stationären Alltag eher intuitiv und entlang von Präferenzen betreffend Arbeitszeit des beantragenden Arztes sowie ökonomischer Faktoren der Abteilung, als unbedingt nach dem Sozialgesetzbuch. Im ambulanten Bereich bestehen durchaus „kunden“-bedingte Konstellationen, die es dem niedergelassenen Arzt gelegentlich bspw nicht.möglich machen, eine an sich nicht indizierte Rehabilitation auch tatsächlich nicht zu beantragen. Gleichzeitig haben wiederum die Kostenträger durchaus auch ökonomische Begrenzungen, die ein Spannungsfeld hinsichtlich der Bewilligung erzeugen.

Bei der Würdigung der Rehabilitationsziele spielen mögliche Zielerreichungsgrade eine große Rolle. Das Maximalziel aller Beteiligten, die vollständige Wiederherstellung der Aktivitäten und Teilhabe gelingt leider nicht allzu oft und wenn, dann meistens im traumatologisch/orthopädischen Bereich. In den internistischen, kardiologischen oder neurologischen Indikationsbereichen ist nicht selten schon der zweitbeste Zielerreichungsgrad, die weitgehende Wiederherstellung mit geringen Defiziten kaum erreichbar. Viel häufiger sind hier bereits als dritter Zielerreichungsgrad Kompensationsstrategien notwendig, also bspw das Training der nicht dominanten Hand bei bleibender Funktionsstörung der Gebrauchshand, oder schließlich als letztes Ziel die Adaptation der Umweltbedingungen an die Funktionsstörungen der Betroffenen um bspw einem Gehunfähigen mit Hilfe von Rollstuhl und weiteren Hilfsmitteln die Teilhabe am sozialen Leben zu ermöglichen.

Im Bewilligungsalltag der Rehabilitation spielen die anzustrebenden Ziele eine durchaus große Rolle. Während Kostenträger unter Kostendruck die Ziele eher am unteren Ende des Erreichbaren einschätzen, dürften die Rehabilitationsträger und die Patienten samt der Angehörigen mehr am oberen Ende prognostizieren bzw hoffen.

\section{Medizinische Voraussetzungen der Rehabilitation}

Die Rehabilitationsbedürftigkeit als Grundvoraussetzung einer Rehabilitationsbehandlung ist oft klarer objektivierbar und konsensfähig als die weiteren Kriterien Rehabilitationsfähigkeit und Rehabilitationsprognose. Ein Funktionsdefizit als Abweichung vom „Normalzustand“, das jeder aus eigener Anschauung nachvollziehen kann, ist relativ leicht erkennbar.

Schwieriger kann es hingegen werden bei der Feststellung der Rehabilitationsfähigkeit, also bei der Einschätzung von Motivation, Motivierbarkeit und Belastbarkeit für die Teilnahme an einer geeigneten Rehabilitation. Bei alten Menschen in geriatrischen Situationen, bei Personen mit psychischen Begleitproblemen aber auch bei verstecktem Rentenbegehren kann die Rehabilitationsproblemen aber auch bei versteck
fähigkit sehr eingeschränkt sein.

Aus Sicht des Autors stellt die Rehabilitationsprognose, also die realistische Einschätzung der Zielerreichung die höchsten Anforderungen und kann fast nur im ärztlichen Bereich ausreichend beurteilt werden. Sie muss auf Basis fundierter Kenntnis der Krankheit und des bisherigen Verlaufs, der Einschätzung des Kompensationspotentials und der Rückbildungsfähigkeit sowie der individuellen biologischen Ressourcen des Patienten entschieden werden.

Bei Betroffensein mehrerer Organsysteme oder Funktionsbereiche, wie nach Schädelhirnverletzung oder bei fortschreitenden Erkrankungen, wie bspw der Multiplen Sklerose oder der Parkinson'schen Erkrankung, treten Schwierigkeiten auf. Ein hirntraumatisierter Patient kann erst nach Monaten relevante Fortschritte zeigen und dies oft nur, wenn in der Zwischenzeit eine durch Bettlägerigkeit und Inaktivität bewirkte Verkümmerung von motorischen und geistigen Leistungen durch intensive, aber zunächst frustran erscheinende, Rehabilitationsanstrengungen verhindert wird. Ein reines medizinisch und pflegerisch begleitetes Zuwarten bis sich Rehabilitationsansätze zeigen würde hier zu nich wieder gut machbaren Schäden führen. Die Rehabilitation muss zunächst unmittelbar beginnen und erst bei im Verlauf, bei praktisch bestätigter Erfolglosigkeit, kann dann ggf ein Abbruch der Rehabilitationsbemühungen und ein zunächst vorübergehendes Zuwarten ohne Intensivrehabilitation erfolgen.

Bei fortschreitenden Erkrankungen wiederum stellt sich die Frage, wie oft und mit welcher Dauer man Rehabilitationsbemühungen für vorübergehende Verbesserungen investieren soll. Denn eigentlich verschlechtern sich derartige Erkrankungen laufend, auch während der Rehabilitation, und das Verhältnis zwischen Einsatz und Erfolg wird meist immer ungünstiger. Im Grenzfall wären solche Patienten praktisch ununterbrochen rehabilitationsbedürftig. Ähnliche Probleme zeigen sich bei Menschen im hohen Alter bzw bei Patienten mit biologisch deutlich eingeschränkten Ressourcen.

\section{4. „Interessen” an der Rehabilitationsbehandlung}

Jenseits der formalen und medizinischen Kriterien lohnt es sich, die mehr subjektiven Interessen der am Rehabilitationsprozess beteiligten Parteien gesondert zu betrachten. 
Besonders unter dem Druck der neu eingeführten pauschalen stationären Vergütungen in Deutschland (DRG-System), aber auch bereits unter dem früheren Druck, die Liegetage zu reduzieren, hat das Akutkrankenhaus ein hohes Interesse, den Rehabilitanden möglichst zügig in die Zuständigkeit des Rehabilitationsträgers zu verlegen. Rehabilitationsanträge mit Datum des Tages der Operation, bspw anlässlich einer Hüftgelenksoperation, sind keine Seltenheit mehr, um dann den Patienten, möglichst schon wenige Tage nach der Operation, noch mit liegendem Nahtmaterial und einer gehörigen Portion Hoffnung auf unkomplizierte Heilung, zu verlegen. Auch neurologische Rehabilitationsanträge, gestellt zum Zeitpunkt der Frühphase eines Schlaganfalles ohne den Akutverlauf abzuwarten, die dann in eine teuere Rehabilitationsform mit hohem Ressourcenverbrauch steuern, resultieren aus dem Kostendruck.

Die Rehabilitationskliniken wiederum müssen auf ihre Auslastung achten und haben insofern kaum ein Interesse, einen angebotenen Patienten je abzulehnen. Allenfalls bei erkennbarem hohem Kostenrisiko, also wenn Komplikationen drohen, wird eine Rehabilitationsklinik gelegentlich auf eine frühzeitige Aufnahme verzichten wollen.

Die Angehörigen benötigen oft bei einem Akutereignis Zeit, um angesichts einer zu erwartenden Behinderung die häuslichen oder pflegeinstitutionellen Voraussetzungen schaffen zu können. Eine lange Liegezeit in der Rehabilitation kommt hier durchaus entgegen, abgesehen natürlich von der Hoffnung auf möglichst weitgehende Wiederherstellung. Bei chronischen Erkrankungen bedeutet eine Rehabilitationsbehandlung eine oft dringend benötigte Entlastung von Pflegearbeit und Stress für die Angehörigen.

Auch der Patient selbst hat bei Akutereignissen das wohl größte und berechtigte Interesse an einer Wiederherstellung oder Besserung und hegt große Erwartungen in die Rehabilitation. Bei chronischen Krankheiten kann die Rehabilitation für ihn eine Abwechslung vom tristen Alltag bedeuten, vergleichbar mit dem Erholungsurlaub eines AN.

Alle diese "Interessenten“, Akutklinik, Rehabilitationsklinik, Angehörige und Patient selbst tendieren hin zu einer möglichst langen, häufigen und hochqualifizierten Rehabilitation. Allein der Kostenträger hat gegenteilige Tendenzen, sicher nicht aus niederen Beweggründen, sondern vielmehr aus rational wirtschaftlichen Erwägungen unter dem bekannten Kostendruck.

\section{Das Phasen-Modell in der Neurologie}

Zum Zweck der bedarfsangepassten Rehabilitation wurde in Deutschland unter dem Dach der Bundesarbeitsgemeinschaft für Rehabilitation (BAR) ein Phasenmodel entwickelt. Ziel ist dabei, die sehr unterschiedlichen und relativ gu abgrenzbaren Heilungsphasen bei neurologischen Erkrankungen zu beschreiben. Für Details sei auf die Veröffentlichungen der BAR und deren Internetauftritt unter http://www.bar-frankfurt.de verwiesen. Anhand der Abbildung soll das Prinzip hier kurz erläutert werden:

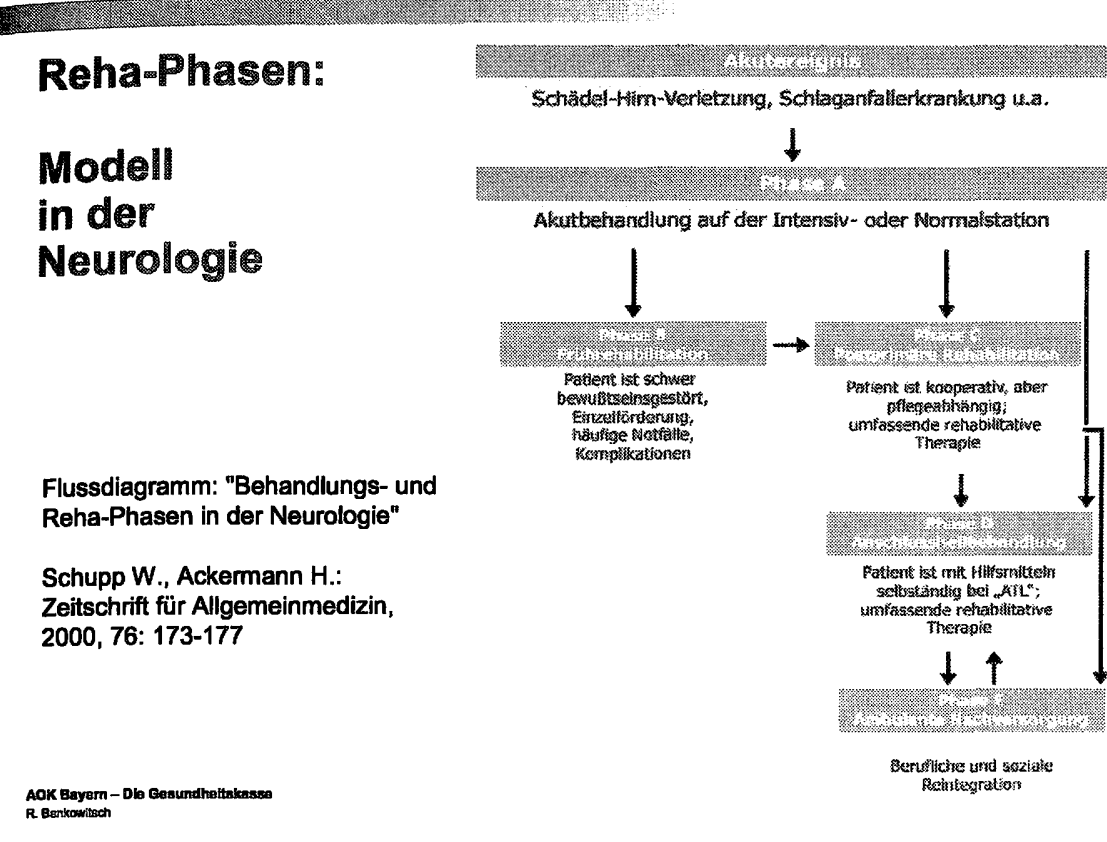

Als neurologische Erkrankungen werden dabei alle schädigenden Ereignisse im Bereich des Gehirns, des Rückenmarks und der peripheren Nervenbahnen verstanden und zwar unabhängig davon, ob durch ein externes (Unfall-)Ereignis oder durch eine Erkrankung im engeren Sinne entstanden.

Phase A: Die Akutversorgung geschieht in der Phase A, also dem Akutkrankenhaus und erfordert dessen typische Vorhaltungen an Diagnostik, Intervention und Pflege. Bereits hier kann eine Frührehabilitation beginnen, bspw auf der Intensivstation, sofern es die Behandlungssituation zulässt. Voraussetzung ist dabei, dass die Klinik ein entsprechendes Rehabilitationsteam besitzt. Das Konzept nach $\$ 39$ Abs. 1 Satz 3 SGB V sieht vor, dass die akutstationäre Behandlung auch die im Einzelfall erforderlichen und zum frühestmöglichen Zeitpunkt einsetzenden Leistungen zur Frührehabilitation umfassen soll. Diese Frührehabilitation verläuft also unter dem Motto „Rehabilitation soweit die Akutsituation es zulässt“.

Phase B: Wenn der Patient medizinisch stabilisiert ist, die absehbaren operativen Eingriffe vorüber sind, er aber schwer bewusstseinsgestört, bettlägerig pflegebedürftig und insgesamt in einem komplikationsträchtigen Zustand ist, kann in der Phase B die Frührehabilitation aufgenommen oder fortgeführt werden. Hier steht nun die Rehabilitation im Vordergrund, eine allfällige akutmedizinische Interventionsfähigkeit muss aber vorhanden sein. Formal und leistungsrechtlich handelt es sich hierbei nach wie vor um eine Akutbehandlung nach $\$ 39$ SGB V. Aus organisatorischen Gründen ist diese Phase B aber fast ausschließlich (in Bayern zu $100 \%$ ) gemeinsam mit den Rehaphasen C und D in einer von der Akutklinik getrennten Rehabilitationseinrichtung realisiert. 
Phase C: Erreicht der Patient ein waches, kooperatives Stadium, in dem er in Kleingruppen und für mehrere Therapiesitzungen täglich belastbar ist und sind akutmedizinische Vorhaltungen wie bspw Monitorüberwachung, assistierte Beatmung mehrfach tägliche ärztliche Visite verzichtbar, so kann er in die Phase $C$ verlegt werden. Der pflegerische und rehabilitative Aufwand ist mit der Phase $\mathrm{B}$ vergleichbar. Hier ist nun also erstmalig die akutmedizinische Vorhaltenotwendigkeit nicht mehr gegeben. Durchaus mögliche Situationen, die ein akutmedizinisches Eingreifen notwendig machen, kommen zwar vor, sind aber absehbar nicht zu erwarten. Sie sind dennoch häufiger als in der „normalen“ Rehabilitation (der Phase D), jedoch bei weitem nicht an der Tagesordnung und müssen ggf durch Konsiliardienste oder Verlegungen, etwa vergleichbar mit Heimpatienten, abgewickelt werden.

Phase D: Erlangt der Patient schließlich die Selbständigkeit in den Aktivitäten des täglichen Lebens (ATL) weitgehend zurück, so kann er in die Phase D verlegt werden. Dies ist die "normale“ Rehabilitation, die Phase nämlich, in der weitestgehend alle anderen, nicht-neurologischen Erkrankungen rehabilitiert werden. Angefangen vom gebrochenen Bein bis hin zum Asthma bronchiale. Die Patienten können sich im Alltag weitgehend selbst versorgen und benötigen meist nur im Bereich des zu behandelnden Organs oder Körperteils Hilfe.

Die anschließenden Phasen E (ambulanten Nachversorgung) und F (zustandserhaltende Maßnahmen) sollen hier nur als Stichwort erwähnt werden.

Die Phaseneinteilung beruht also auf klinischen Merkmalen des Patienten, insbesondere auf der Notwendigkeit und der Priorität von akutmedizinischen Interventionen (Abgrenzung der Phasen A und B von $C$ ), erst sekundär auf dem Rehabilitationsaufwand (Abgrenzung der Phasen $C$ von D) und kaum auf zeitlichen Aspekten im Verhältnis zum Akutereignis. Grundsätzlich können einzelne Phasen, ausgehend von der Akutversorgung, übersprungen werden oder es kann die Rehabilitation in einer Phase stecken bleiben und dann ggf direkt in eine Pflegebehandlung einmünden.

\section{Einordnung der geriatrischen Rehabilitation}

Ergänzend muss noch erläutert werden, dass es typischerweise ältere, multimorbide Patienten, mit oft sehr weit eingeschränkten biologischen Ressourcen gibt, die mit oder ohne neurologischen Erkrankungen einer Rehabilitation bedürfen, die jedoch nicht selbständig genug für die „normale“ Rehabilitation in der Phase D sind. Hier übernimmt die geriatrische Rehabilitation die Versorgung, sie beginnt sobald die Akutbehandlung abgeschlossen ist. Die Patienten befinden sich im klinischen Zustand der Phasen C und D, teilweise auch noch der Phase B. Rehabilitationsziel ist in aller Regel die möglichst weitgehende Wiederherstellung der Selbständigkeit im Alltag.

\section{Begriff der Frührehabilitation}

Bezüglich des Begriffes „Frührehabilitation“ herrscht teils große Unklarheit Manchmal wird nur im Fall der Rehabilitation im Rahmen der Phase A von Frührehabilitation gesprochen, dann wiederum bei allen Rehabilitationsbe- mühungen, die zeitlich früh nach dem Ereignis beginnen und schließlich noch bei der Rehabilitation in den Phasen B und/oder C. Es sind in Bayern sogar Gerichtsverfahren anhängig, die auf dieser Begriffsverwirrung beruhen und von deren Unklarheiten sich Rehabilitationsträger Ansprüche ableiten wollen. Im Sinne einer klaren Sprachregelung könnte man somit in der Phase A von Akutrehabilitation, also der Rehabilitationsbehandlung im Akutkrankenhaus sprechen, in der Phase B von der eigentlichen Frührehabilitation, in der Phase C von der weiterführenden Rehabilitation. Jedenfalls sollte das Attribut "Früh“ in Verbindung mit der Rehabilitation nicht zeitlich verstanden werden, ansonsten sind Fehleinweisungen und Missverständnisse vorprogrammiert.

\section{Phasenmodell nur geeignet für neurologische Erkrankungen}

Für nicht-neurologische Erkrankungen eignet sich ein Phasenmodel eigentlich nicht. Denn die Patienten sind meist nur lokal im Bereich einer Extremität oder eines Organs betroffen und sind im Alltag, abgesehen von der Organstörung, selbständig. Sie weisen ja gerade keine neurologische Störung als häufigste Ursache einer Unselbständigkeit auf bzw sie sind nicht multimorbide im geriatrischen Sinne. Die Heilung verläuft auch meist monophasisch, wenn man von Wundkomplikationen oder interkurrenten Zusatzerkrankungen absieht.

Dennoch finden sich auch bei den nicht-neurologischen Rehabilitationsfällen einige Problemfelder bezüglich Beginn und Ende bzw Nachhaltigkeit, die später anhand von Beispielen erläutert werden.

\section{Informationsquellen}

Abschließend zum allgemeinen Rahmen soll hier noch auf Informationsquellen hingewiesen werden. Wie bereits erwähnt, finden sich auf den Internetseiten der BAR unter der Rubrik „Publikationen“ vielfältige Empfehlungen mit sehr praktischen Angaben zu einzelnen Rehabilitationsformen (ambulant, stationär) und zu praktisch allen Indikationsbereichen.

Weiterhin sei der Wegweiser der BAR empfohlen, den es kostenlos als Date gibt (http://www.bar-frankfurt.de/upload/BAR_Wegweiser_142.pdf) oder auch als Druckprodukt im Buchhandel. In diesem sehr nützlichen Werk sind sämtliche Aspekte des Rehabilitationswesens anschaulich geschildert und für Laien gut zugänglich.

\section{Nachhaltigkeit von Rehabilitation - Praxisschock}

Ein Punkt, der den Rahmen hier sprengen würde, sei nur als Thema erwähnt: Die Nachhaltigkeit von Rehabilitationsanstrengungen. Nicht selten tritt im Anschluss an die erfolgreich verlaufene Rehabilitation bei den Rehabilitanden der so genannte „Praxisschock“ auf, also die „Konfrontation mit dem Arbeitsalltag und Familienalltag begleitet vom Verlust der Motivation zur Lebensstiländerung" so ein Zitat im Leitfaden der Deutschen Rentenversicherung. Tatsächlich entlässt unser Gesundheitssystem Rehabilitanden nicht selten nach Erreichen des individuellen Rehabilitationsziels nach Hause, ohne sich nachhaltig um die 
weiteren Entwicklungen zu kümmern. Der Ubergang in den Alltag stellt aber oft bereits bei nur geringen Funktionseinschränkungen eine Quelle der Frustration und Demotivation für den Betroffenen dar, die zu Depression und Verlust bereits erreichter und möglicher zukünftiger Fähigkeiten führt. Kostbare Investitionen der Akutmedizin und der Rehabilitation in den Betroffenen gehen dadurch verloren, sehr zum Schaden des Betroffenen selbst und der Solidargemeinschaft. Hier wären nachhaltige Anstrengungen eine sehr wünschenswerte Entwicklung.

\section{Sammlung von Fallbeispielen der medizinischen Rehabilitation}

Im Folgenden soll nun anhand von praktischen Beispielen das zuvor Beschriebene veranschaulicht werden.

\subsection{Erster Normalfall: Abfolge Akutbehandlung - Rehabilitation}

Beispiel: Arbeitsunfall mit kompliziertem, offenem Beinbruch mit Beteiligung des Kniegelenkes.

In der Akutbehandlung findet statt: Operation des Bruches, Beherrschung der Wundinfektion, Stabilisierung der Wundverhältnisse, erste krankengymnastische Übungen in der Akutklinik (Phase A). Danach folgt die Verlegung verzögerungsfrei in eine traumatologische Rehabilitationseinrichtung.

Im stationären Bereich in unfallchirurgisch/orthopädischer RehabilitationsKlinik (Phase D) finden intensive Krankengymnastik mit Gelenkmobilisierung Wundkontrolle und Mobilisierung im Alltag statt. Der Patient ist im Alltag weitgehend selbständig. Im Anschluss sodann folgt im ambulanten Bereich die zunehmende Mobilisierung bis zur Vollbelastbarkeit. Zu späterem Zeitpunkt wird ggf die Metallentfernung vorgenommen, teils vor, teils nach Wiederaufnahme der Berufstätigkeit.

\subsection{Zweiter Normalfall: Abfolge Akutbehandlung - Frührehabilitation}

Beispiel: Junger Mann mit Motorradunfall, „Polytrauma“ mit Hirnprellung und Einblutung, Lungenquetschung, mehrfachem Beinbruch.

In der Akutklinik finden die Versorgung mittels Intensivstationsbehandlung, künstlichem Koma, Operation der Brüche, Drainage des Lungenfellergusses und der Lungeneinblutung sowie eine Hirnschwellungsbehandlung statt. Auf der Intensivstation wird bereits mit Akutrehabilitation begonnen, wie oben erläutert von einem speziellen Rehabilitationsteam nach $\$ 39$ SGB V (Phase A). Nach einer Liegezeit von ca 6 Wochen auf der Akutintensivstation ist schließlich das künstliche Koma aufgehoben, der Patient atmet spontan durch einen Luftröhrenschnitt, nimmt ansatzweise Blickkontakt auf.

Die Rehabilitation erfolgt entlang dem Phasenmodell: Zunächst akutstationär in Form der Versorgung in einer neurologischen Rehabilitations-Klinik (Phase B, Einrichtung mit vorhandenen Vorhaltungen der Akutmedizin). Hier werden intensive Rehabilitationsbemühungen zur Wiederherstellung von Kooperationsfähigkeit und medizinischer Stabilität betrieben, die Bewältigung von
Blutdruck-Krisen bei Mobilisierung und Monitorüberwachung 24 Stunden täg lich sind lebenswichtig. Nach 8 Wochen erfolgt die Verlegung in die Phase $C$ (Vorhaltungen der Akutmedizin nicht mehr erforderlich). Der Patient ist kreis laufstabil, hat keine Krisen mehr, der Luftröhrenschnitt wurde verschlossen, die Atmung und das Schlucken gelingen problemlos, es kann oral ernährt werden, der Patient ist im Pflegerollstuhl mobilisiert.

Es handelt sich um einen klassischen neurologischen Schwerst-Rehabilitations-Fall. Der Verlauf erscheint weitgehend unkompliziert, dabei durchläuft der Patient alle Rehabilitationsphasen bis E.

\subsection{Versorgungsproblem: Mangelnde Rehabilitation} während Akutbehandlung

Beispiel: Älterer Patient mit Lungenentzündung bei Zuckerkrankheit, $\mathrm{Ge}$ lenkverschleiß, bislang Selbstversorgung in eigener Wohnung mit Gehstock.

Die Akutbehandlung umfasst eine antibiotische Behandlung inklusive Infusionen und Mitbetreuung der Zuckerkrankheit. Es findet täglich mobilisierende Krankenpflege statt, zusätzlich drei Mal wöchentlich 20 Minuten Krankengymnastik. Der Patient ist insgesamt 16 Tage lang bettlägerig (drei Tage zuhause mit gezählt). Er kann schließlich nur mit Hilfe in den Pflegestuhl bewegt werden, is somit im Alltag hilfsbedürftig. Es ist klar, dass eine Rehabilitation angezeigt ist Diese erfolgt stationär in einer geriatrischen Rehabilitationsklinik. Nach intensiven Mobilisierungsbemühungen kann der Patient nach drei Wochen mit einem Rollator nach Hause entlassen werden. Ob die an sich iS einer Nachhaltigkeit zuhause erforderliche Krankengymnastik und ein Konditionstraining unter streng budgetierten Heilmittelressourcen erfolgen, ist fraglich.

Aus rehabilitationsmedizinischer Sicht beginnt die Rehabilitation zu spät, die ambulante Rehabilitation nach Entlassung nach Hause dürfte in aller Regel mangelhaft sein, sofern sie je stattfindet. Jede Bettlägerigkeit bei älteren Menschen die länger als nur wenige Tage anhält, erhöht die Gefahr, dauerhaft ein Stück Unabhängigkeit zu verlieren. Insofern wäre auch auf der internistischen Normalstation eine "Akutrehabilitation“ als Standard notwendig. Die mobilisierende Krankenpflege und Krankengymnastik auf der Akutstation genügen nach eigener Erfahrung fast nie, um die Konditionsverluste bei älteren Patienten aufzuhalten. Es wäre ein spezielles Assessment "drohende Pflege“ und eine klare Akutrehabilitations-Interventionsstrategie sinnvoll. Das Ziel sollte sein, dass jeder ältere Patient spätestens nach dem dritten Liegetag täglich (auch am Wochenende!) von einem Rehabilitationsteam versorgt wird.

\subsection{Versorgungsproblem: Fehler in der Akutbehandlung -} dadurch hoher Rehabilitationsaufwand

Beispiel: Älterer Patient mit Schlaganfall, leichte Halbseitenlähmung, desorientiert, kann alleine nicht essen oder trinken, ist harninkontinent. Bislang Selbstversorgung in eigener Wohnung.

Die Akutbehandlung scheint von außen gesehen schicksalhaft: Es erfolgen Infusionsbehandlungen über Tage hinweg, allerdings erhält der Patient bereits 
am ersten Tag Breikost in dem er von der Stationshilfe gefüttert wird. Aufgrund einer Schluckstörung durch den Schlaganfall kommt es zu Verschlucken und durch die Nahrungsbestandteile in der Lunge zu einer eitrigen Lungenentzündung. Diese muss mit Antibiotika 14 Tage lang behandelt werden. Zwischenzeitlich ist der Patient durch die Komplikation schwer verwirrt, er ist nach insgesamt 21 Tagen Bettlägerigkeit schließlich im Pflegestuhl neben dem Bett mobilisiert.

Die Rehabilitation erfolgt stationär in einer geriatrischen Rehabilitationsklinik. Dort finden intensive Mobilisierungsbehandlungen statt, nach drei Wochen kann der Patient mit Unterstützung gehen, kommt aber zuhause alleine nicht mehr zurecht. Die Unterbringung in einem Pflegeheim ist unumgänglich. Dort wäre eigentlich weitere Krankengymnastik dringend angezeigt. An deren konsequenter Umsetzung kann gezweifelt werden.

Der Beginn mit Breikostfütterung ohne Schluckdiagnostik auf fachkompetenter Basis ist häufig Ursache von Lungenentzündungen im Akutbereich. Sie stellt einen iatrogenen, vermeidbaren Schaden dar, der schwere Folgen nach sich zieht. Ohne die lange Immobilisierung wäre es vermutlich nicht zu dem Verlust der Mobilität gekommen, ganz zu schweigen von der Gefährdung durch die Lungenentzündung. In der Folge genügt die mobilisierende Pflege im Akutbereich nicht um den Immobilisierungs-Schaden zu kompensieren. Wie im vorigen Fall wäre hier ein spezielles Assessment „drohende Pflege" und eine Akutrehabilitations-Interventionsstrategie angezeigt.

\subsection{Abgrenzungsproblem: Akutbehandlung - stationäre -}

\section{ambulante Rehabilitation}

Beispiel: Pflegeheimbewohner, nicht mehr alleine gehfähig mit vorbestehender Halbseitenlähmung bei Zuckerkrankheit erleidet neuerlichen Schlaganfall mit Sprachstörung und Verschlechterung der Lähmung.

Die Akutbehandlung erfolgt mit Routine-Diagnostik und Ausschluss behandlungsbedürftiger, sonstiger Erkrankungen. Der Patient kann nach zwei Wochen im Mobilisierungs-Rollstuhl am Bettrand mobilisiert werden, kann wieder sprechen. Es folgt eine Rehabilitationsbehandlung in einer geriatrischen Rehabilitation, dem beantragenden Stationsarzt ist der Vorzustand nicht bekannt. Im Rehabilitationsantrag wird die Rehabilitationsprognose überschätzt, die Angehörigen drängen sehr auf Rehabilitation.

In der Rehabilitation wird innerhalb von fünf Wochen erreicht, dass der $\mathrm{Pa}$ tient mit dem Rollator wenige Schritte gehfähig ist, die Sprache ist wie schon im Rahmen der Akutversorgung kein Problem mehr. Zurück im Pflegeheim kann der Patient nach weiteren 2 Wochen nicht mehr mit dem Rollator gehen, er sitzt den ganzen Tag, schafft mit Hilfe die Transfers ins Bett, insgesamt ist der Status wie vorher.

Hier muss auffallen, dass die geriatrische Rehabilitation ohne tragfähiges Rehabilitationsziel beantragt wurde. Es konnte durch intensive Rehabilitationsbemühungen zwar eine Verbesserung der Mobilität erreicht werden. Diese kann jedoch im Alltag nicht gehalten werden. Vermutlich wäre eine Rückverlegung in die Pflegeeinrichtung mit (intensiver und längerfristiger) ambulanter Krankengymnastik effektiver, günstiger und ausreichend gewesen.
11.6 Abgrenzungsproblem: Rehabilitation Phase C - D - ambulant

Beispiel: 68 jähriger Patient mit Schlaganfall, bisher vollkommen selbständig, sportlich aktiv. Deutliche Halbseitenlähmung, Sprechunfähigkeit, Schläfrigkeit, hohe Blutdruckwerte.

Die Akutbehandlung verläuft unkompliziert: Rasche Diagnostik, Infusionstherapie. Am dritten stationären Tag wird eine neurologische Rehabilitation Phase $\mathrm{C}$ beantragt. Die Verlegung erfolgt am neunten Tag nach dem Ereignis. In der stationären Rehabilitationsklinik der Phase C erscheint der Patient als Fußgänger mit Gepäck in Begleitung des Ehepartners. Die Rehabilitationsklinik nimmt in die Phase $\mathrm{C}$ auf, nachdem in der Phase $\mathrm{D}$ kein Bett frei ist, verlegt nach drei Tagen in die Phase D. Nach drei Wochen von dort Entlassung nach Hause mit minimaler Restlähmung, die Sprachfähigkeit ist wieder vollständig zurückgekehrt.

Objektiv wurde die Rehabilitationsbehandlung viel zu früh beantragt, noch bevor Stabilisierung und Akutverlauf absehbar waren. Dadurch wurde dem $\mathrm{Pa}$ tienten eine klar $\mathrm{zu}$ hochwertige, somit unwirtschaftliche Rehabilitationsform zugeordnet. Da sich in vielen Fällen beim Hirninfarkt nach einigen Tagen die zunächst schweren Ausfälle zurückbilden, sollte der Rehabilitationsantrag erst nach Stabilisierung gestellt werden. Im vorliegenden Fall hätte von Anfang an die Phase D oder gar eine ambulante Rehabilitation ausgereicht. Der finanzielle Druck in den Akutkliniken, teils verbunden mit einer gewissen Unerfahrenheit der antragstellenden Instanzen, führt hier nicht selten zu Verschwendung.

\subsection{Abgrenzungsproblem: stationäre bzw ambulante Rehabilitation}

Beispiel: 70 jähriger Patient, akuter Herzinfarkt, leichter Bluthochdruck, Blutfette erhöht

In der Akutbehandlung erfolgt eine Herzkatheterbehandlung mit Aufdehnung der Engstelle der Herzkranzgefäße, es sind nur geringe Schäden am Herzen entstanden. Es folgt die Rehabilitation stationär in einer kardiologischen Rehabilitationsklinik. Nach drei Wochen von dort Entlassung nach Hause ohne Beschwerden. Es ergeht die Empfehlung an einer Herzsportgruppe teilzunehmen.

Die unkomplizierte stationäre Rehabilitation ist routinemäßig durchgeführt worden, eigentlich ohne zwingende medizinische Notwendigkeit. Im Fall eines sehr unwahrscheinlichen Ereignisses von Herzflimmern bestünden möglicherweise forensische Probleme. Somit entspringt der stationäre Rehabilitationsantrag auch dem Motiv der Abwehr forensisch bedingter Klagen. Eine intensive ambulante Rehabilitation wäre vermutlich effizienter.

\subsection{Versorgungsproblem: Wahleingriff Gelenkersatz -} fehlende vorausgehende Rehabilitation

Beispiel: Älterer Patient mit jahrelang sich verschlimmernder Hüftgelenksarthrose, insgesamt altersschwächlicher Patient, Zuckerkrankheit, bislang alleine lebend.

Die Akutbehandlung beginnt mit der Aufnahme in eine Orthopädie zum Hüftgelenksersatz. Nach problemloser Operation, kommt der Patient nur mit vie Unterstützung aus dem Bett, er kann nur im Gehwagen mit Unterstützung durch 
zwei Hilfspersonen mobilisiert werden. Aufgrund der eingeschränkten Selbständigkeit im Alltag erfolgt die Verlegung in eine geriatrische Rehabilitationsklinik, $\mathrm{da}$ in der orthopädischen Rehabilitationsklinik Selbständigkeit im Alltag erforderlich ist. Nach 6 Wochen Rehabilitation kann Patient mit Rollator nach Hause entlassen werden. Ambulante Krankengymnastik und Konditionstraining sind wie so häufig notwendig, die Umsetzung kann Schwierigkeiten bereiten.

Medizinisch gesehen ist die Indikation zur Operation des Hüftgelenksschadens korrekt. Aber: Eigentlich hätte der Patient vor der Operation mindestens zwei Wochen intensive Konditionierung benötigt, mit Erlernen des Krückengehens. Besser und kostengünstiger wären 10 Wochen ambulante Vorbereitung für die postoperative Phase gewesen. Bei entsprechender Kräftigung und Mobilisierung der rückgebildeten Hüftmuskulatur und Einübung des Gehens mit Krücken hätte die Rehabilitation wesentlich besser verlaufen können. Analog zum Sport bei Gesunden sollten auch vorbelastete Menschen vor Wahleingriffen trainiert werden. Neben einer Verkürzung der Rehabilitationsdauer und Vermeidung von Immobilisierungskomplikationen könnte hier sogar eine Kosteneinsparung an anderer Stelle entstehen: Möglicherweise entscheiden sich Betroffene Patienten mit Arthrose der großen Gelenke nach einer adäquaten Rehabilitationsbehandlung und intensiver Trainingsphase sogar für ein Vorgehen ohne Operation nicht selten sind danach nämlich die Beschwerden erheblich gebessert.

\section{Zusammenfassung}

Einleitung und Bewilligung von Rehabilitationsbehandlungen erfordern fundierte ärztliche Beurteilungen auf Basis belastbarer Kenntnisse der medizinischen Grundlagen, der Sozialgesetzgebung und der Versorgungsstrukturen. Dabei existieren teils erhebliche "Interessenlagen“, die einer streng medizinisch begründeten Rehabilitation entgegen stehen können. Solange die Vorhaltungen der Akutmedizin zur Behandlung oder Überwachung wegen allfälliger Notsituationen angezeigt sind, findet die neurologische Rehabilitation unter dem Dach der Akutmedizin statt (Phasen A und B). Sobald keine vital bedrohlichen Komplikationen mehr absehbar sind, können neurologische Patienten in Phasen C D, E, F oder ambulant weiter rehabilitiert werden. Es existieren Defizite an Rehabilitationskapazität im Bereich der Akutkliniken, betreffend die „Frührehabilitation" nach $\$ 39$ SGB V. Dies gilt für die Intensivstationen der vielen Akuthäuser aber noch mehr für die stationäre Normalversorgung bei alten Menschen. In der präoperativen Phase bei Wahleingriffen an großen Gelenken, in der Nachhaltigkeitssicherung im häuslichen Bereich und im Bereich der ambulanten Rehabili tation sind teils noch nicht einmal Ansätze vorhanden.
Johannes Rudda

\section{Das System der Rehabilitation in Österreich}

\section{Das System der Rehabilitation in Österreich 1.1 Historische Entwicklung}

Historisch gesehen wurde die stationäre medizinische Rehabilitation zunächst in der sozialen UV entwickelt. Das älteste Rehabilitationszentrum (Stollhof/Klosterneuburg) geht auf die in den 30er Jahren des 20. Jahrhunderts von Prof. Lorenz Böhler entwickelte Unfallchirurgie zurück und diente einer schon damals als unerlässlich erkannten Nachbehandlung. Seit dieser Zeit hat die AUVA eine Reihe moderner Unfallspitäler und Rehabilitationszentren errichtet, die derzeit bereits zu $80 \%$ durch Personen, die Freizeitunfälle erlitten haben und nur zu $20 \%$ mit Versehrten nach Arbeitsunfällen oder Berufskrankheiten frequentiert werden. Die UV der AN, Gewerbetreibenden und neuen Selbstständigen ist im ASVG geregelt ( $\$ \$ 4,7-8,19-20,172-220,476-477)$ jene der Bauern im BSVG ( $\$ 3,7,11,148$ - 149s) und die der Beamten im B-KUVG $(\$ \$ 1,3,87-117)$.

Der Gesetzgeber sieht im Abschnitt VI des ASVG idF der 9. Novelle ( $\$ 300$ - 307f) unter dem Titel „Rehabilitation und Maßnahmen der Gesundheitsvorsorge" Bestimmungen vor, die im Bereich der sozialen PV Regelungen für die meisten Rehabilitanden und Kurpatienten treffen. Diese Normen sind auch Ausdruck für die Bemühungen von Rehabilitationsmedizinern seit 1960 in den PV-Trägern sowohl aus ökonomischen als auch aus humanitären Aspekten die Invalidität (Berufsunfähigkeit) von Arbeitern und Angestellten bei bestimmten Indikationen (zB Herz-Kreislauferkrankungen, Erkrankungen des Bewegungsund Stützapparats) zurückzudrängen, was teilweise auch erfolgreich bewirkt werden konnte. Parallelbestimmungen finden sich später auch im GSVG (Abschnitt IV, \$\$157-171) und im BSVG (Abschnitt V, \$\$150-163) mit dem gleichen Ziel, die Erwerbsunfähigkeitspensionen bei Selbstständigen zu verringern. Ebenso sind im B-KUVG gleichartige Maßnahmen unter dem Begriff „erweiterte Rehabilitation" - $\$ 70$ und 70 b leg cit - vorgesehen, um Dienstunfähigkeitspensionen von Beamten zu vermindern.

Schließlich hat der Nationalrat mit der 50. ASVG-Novelle und Parallelnovellen auch in der sozialen KV die medizinische Rehabilitation für Angehörige sowie Alters- und Hinterbliebenenpensionisten beschlossen, um auch für diesen Personenkreis eine Verbesserung oder Stabilisierung des Gesundheitszustandes nach einem Akutereignis zu bewirken ( $\$ 154 \mathrm{a}$ ASVG, $\$ 99$ a GSVG, $\$ 96 a$ BSVG, $\$ 65$ a B-KUVG). Die Absicht des Gesetzgebers ging dahin, vor allem die Selbstständigkeit dieses Personenkreises im täglichen Leben wieder herzustellen, um Pflegebedürftigkeit ganz oder teilweise zu vermeiden. 
1.2 Einrichtungen der Sozialversicherung und privater Rechtsträger

Im Bereich der medizinischen Rehabilitation betrieben im Dezember 2007 alle SV-Träger 29 Sonderkrankenanstalten (Rehabilitationszentren) mit 3.819 Betten. Nach Indikationen sind vor allem Rehabilitationszentren für den Bewegungs- und Stützapparat mit der Zusatzindikation Rheumatologie, solche für Herz- und Kreislauferkrankungen, aber auch für Stoffwechselstörungen (Diabetes) vorherrschend. Seit 2000 wurden auch die Bemühungen auf dem Sektor der Neurologie verstärkt - durch ein neues Rehabilitationszentrum und durch Umwidmung von Betten in bestehenden Rehabilitationszentren.

Bereits im Jahre 1999 hat der Rechnungshof sich über die Führung von Rehabilitationszentren durch die SV sehr positiv geäußert, zumal er hervorhob dass in diesen Einrichtungen keine Riskenselektion getroffen wird. Er sah nämlich die Gefahr bei privaten Betreibern, dass diese aufgrund ihrer betriebswirtschaftlichen Überlegungen schwere Fälle mit hoher Betreuungsintensität wegen der damit verbundenen überproportionalen Personalkosten ausgrenzen würden, sodass dann eine Lücke gerade für jene Menschen entstünde, die die Maßnahmen der medizinischen Rehabilitation am Dringendsten benötigen würden.

Trotz Einsparungsmöglichkeiten im Falle der Privatisierung sollen die Rehabilitationsleistungen aus Gründen der Versorgungssicherheit und Qualitätssicherung weiterhin (auch) in eigenen Einrichtungen der SV-Träger erbracht werden (Empfehlung des Rechnungshofes, Schwerpunktprüfung bei 17 SV-Trägern betreffend die Struktur der eigenen Einrichtungen und deren Ausgliederungs- bzw Privatisierungsmöglichkeiten vom 4. 10. 1999, Zl 001.878/027I/6/99).

Freilich hat der Rechnungshof auch einige Defizite der medizinischen Rehabilitation angesprochen und deren Behebung verlangt. Dies betraf vor allem die Schaffung von entsprechenden Bettenkapazitäten für neurologische, onkologische und psychische Erkrankungen. Ebenfalls sollte ein flächendeckendes System für die ambulante Rehabilitation überlegt werden.

Auch im Jahre 2004 und 2009 hielt der Rechnungshof nach Auskunft eines maßgeblichen Beamten an seiner Grundaussage der Vermeidung einer Riskenselektion in der medizinischen Rehabilitation fest.

Die Dominanz der eigenen Einrichtungen liegt eindeutig bei der PV: 16 Rehabilitationszentren werden von der PVA, 4 Eigene Einrichtungen von der Versicherungsanstalt öffentlich Bediensteter, 4 Sonderkrankenanstalten für medizinische Rehabilitation werden noch von der SVB und 4 Einrichtungen mit Mehrheit von der SVA geführt. Die Sonderkrankenanstalt Bad Gleichenberg für Lungenerkrankungen der SVB wird überwiegend (74\%) mit privaten Gesellschaftern betrieben. Einige hochspezialisierte Einrichtungen hat die AUVA für die neuro- bzw unfallchirurgischen Indikationen in Betrieb.

\subsection{Gesetzliche Ermächtigung zu privatrechtlichen} Beteiligungsmöglichkeiten

$\$ 81$ Abs 2 ASVG, der mit der 58. ASVG-Novelle am 6.7.2001 vom Nationalrat beschlossen und am 1.8.2001 in Kraft getreten ist, sieht vor, dass die SV-
Träger ermächtigt werden, eine Errichtung (Gründung) von oder die Beteiligung an Vereinen, Fonds und Gesellschaften mit beschränkter Haftung im Rahmen von Finanzierungs- und Betreibermodellen, wenn sie der Verbesserung der Servicequalität oder der Erzielung von Einsparungen dient, einzugehen. Unter den gleichen Voraussetzungen ist die Beteiligung von natürlichen oder juristischen Personen an Vereinen, Fonds und Gesellschaften mit beschränkter Haftung, die von einem Versicherungsträger (dem HV) errichtet (gegründet) wurden, zulässig Auch mit Gebietskörperschaften können solche Verträge geschlossen werden.

Gleichlautende Bestimmungen wurden in der 25. GSVG-, 24. BSVG- und 28. B-KUVG-Novelle vorgesehen.

In den EB zur RV zur 58. ASVG-Novelle wird betont, dass in den Fällen, wo öffentliche Verwaltungen über eine hohe eigene Kompetenz im privatwirtschaftlichen Bereich verfügen, die Strategie einer Partnerschaft mit einem privaten Dienstleister verfolgt werden sollte.

Ein erster Schritt sollte als Idealtypus des Gesetzgebers dadurch erfolgen, dass die Versicherungsträger ausdrücklich dazu ermächtigt werden,

- marktwirtschaftliche und innovative Finanzierungs- und Betreibermodelle zur Verbesserung der Service-Qualität und zur Erzielung von Einsparungen auszuwählen,

- dadurch Managementfähigkeiten, die Kreativität und die kommerziellen Fähigkeiten des privaten Sektors für die Bereitstellung von Dienstleistungen im Rahmen der sozialen Sicherheit nutzbar gemacht werden, um letztendlich

- die Kunden- und Serviceorientierung in der SV zu erhöhen sowie den Einsatz neuer Technologien zu forcieren, wobei

- die Bereitschaft des privaten Partners gegeben sein muss, Leistungen, Wertschöpfung und Produktivität zu schaffen, eigenes Kapital zu investieren, Risiko zu übernehmen und Know-how zu teilen,

- und der oder die Partner aus dem privatwirtschaftlichen Bereich jedenfalls im Rahmen einer Ausschreibung auszuwählen sind.

In der folgenden Tabelle werden die zwei wichtigsten privatrechtlichen Gestaltungsmöglichkeiten mit ihren wesentlichsten positiven und negativen Rechtsfolgen dargestellt:

\begin{tabular}{|c|c|c|}
\hline \multicolumn{3}{|c|}{ Alternative Gestaltungsformen für Rehabilitationseinrichtungen } \\
\hline Rechtsform & Vorteile & Nachteile \\
\hline GmbH & $\begin{array}{l}\text { - juristische Person des Handelsrechts } \\
\text { - Haftungsbeschränkung } \\
\text { - Geschäftsführer mit größerem Hand- } \\
\text { lungsspielraum }\end{array}$ & $\begin{array}{l}\text { - Risken der Privat- } \\
\text { wirtschaft } \\
\text { - geringere Arbeits- } \\
\text { platzsicherheit }\end{array}$ \\
\hline Verein & $\begin{array}{l}\text { - einfache Gründung } \\
\text { - leichtere Kooperation mit NGOs }\end{array}$ & $\begin{array}{l}\text { - Haftungsprobleme } \\
\text { der Organe } \\
\text { - Überforderung bei } \\
\text { größeren Einrich- } \\
\text { tungen }\end{array}$ \\
\hline
\end{tabular}


Bislang hat die SVA durch Gründung einer GmbH mit 100\%iger und einer anderen mit 51\%iger Beteiligung, aber Auslagerung des operativen Geschäfts an eine Betriebsgesellschaft, eine privatrechtliche Gestaltungsform durchgeführt. Eine Ausschreibung für ein weiteres Rehabilitationszentrum steht unmittelbar bevor.

Die SVB hat ebenfalls für eines ihrer Rehabilitationszentren (Bad Gleichenberg) eine eigene Betriebsgesellschaft mit einer Sperrminorität des Trägers $(26 \%)$ gegründet und die übrigen Anteile an private Rechtsträger verkauft. Ferner wurden 2008 auf dieser Basis auch die weiteren vier Rehabilitationszentren zum Verkauf ausgeschrieben.

\subsection{Die Rehabilitation im Stufenbau der Rechtsordnung}

Die Rechtswissenschaft hat vor allem durch die Vertreter des Positivismus - maßgeblich ist das gesatzte Recht im Gegensatz zum Naturrecht - einen Stufenbau der Rechtsordnung entwickelt. Als höchste Normen (= oberste Stufe) existieren die Bundesverfassung und alle Gesetze im Verfassungsrang. Darunter sind als weitere Stufe die einfachen Gesetze des Nationalrats (im Bundesrecht) zu qualifizieren, zumal hiefür bei der Beschlussfassung keine qualifizierten Quoren für die Präsenz der Nationalräte bzw Abstimmung erforderlich sind. Eine weitere Stufe tiefer sind die Verordnungen, zu denen Vollzugsorgane, wie zB Bundesminister, vom Gesetz ermächtigt sind. Nach herrschender Judikatur und Lehre sind auch Satzungen der SV-Träger bzw Richtlinien des HV im Rang einer Verordnung. Dabei werden auch nach der Bindungswirkung an alle Rechtssubjekte solche Verordnungen als Rechtsverordnungen und bei einer Bindung nur durch die Vollzugsorgane diese Verordnungen als Verwaltungsverordnungen qualifiziert.

Verfassungsrechtlich ist der Begriff der medizinischen Rehabilitation zweifellos dem Kompetenztatbestand des Art 10 Abs 1 Z 11 B-VG (SV-Wesen) zuzuordnen. In der einfachen Gesetzgebung weist er allerdings eine unterschiedliche Qualität der Normen für die Anspruchsberechtigung auf, was in der Folge bei den einzelnen Zweigen der SV noch erläutert wird.

\subsubsection{Rehabilitationsleistungen der Unfallversicherung}

Die medizinischen Rehabilitationsleistungen der UV (= Wiederherstellung der Gesundheit und Erwerbsfähigkeit mit allen geeigneten Mitteln) sind vom Gesetzgeber als Pflichtleistungen, somit mit individuellem Rechtsanspruch und Bescheidrecht des Versicherten konzipiert. Dies ist insb bei zentralen Bestimmungen des Leistungsrechts der Fall (zB $\$ 189$ ASVG: Die Unfallheilbehandlung hat mit allen geeigneten Mitteln zu erfolgen oder $\$ 199$ ASVG: Der UV-Träger hat dem Versehrten ein Übergangsgeld zu leisten bzw $\$ 203$ ASVG: Anspruch aufVersehrtenrentebeht ...). Obwohl die medizinische Rehabilitation nicht ausdrücklich als solche im Gesetz bezeichnet ist und daher unter die Unfallheilbehandlung zu subsumieren ist, besteht sowohl nach der Rechtsauffassung der AUVA als auch nach jener des HV Übereinstimmung, dass sie eine Pflichtleistung ist, gegen deren Ablehnung ein Bescheid- und Klagerecht des Versicherten vor dem zuständigen Arbeits- und Sozialgericht besteht. Über die Maßnahmen der beruflichen und sozialen Rehabilitation entscheidet jeder UV-Träger selbst Es gibt daher ambitionierte Einrichtungen zur beruflichen Umschulung Versehrter und wirksame Unterstützungen für die Adaptierung von Wohnraum und behindertengerechter Ausstattungen von Kraftfahrzeugen und Arbeitsplätzen.

\subsubsection{Rehabilitationsleistungen der Pensionsversicherung}

Bei den Rechtsvorschriften über die medizinische Rehabilitation durch die PV-Träger (Maßnahmen zur Verhinderung von Invalidität [Berufs-/Erwerbsunfähigkeit]) normiert der Gesetzgeber keinen Rechtsanspruch, sondern sprich lediglich von einer "Gewährung“, was von der Rechtsprechung und Lehre als sogenannte „Pflichtaufgabe“ bezeichnet wurde. Damit besteht zwar eine objektive Leistungsverpflichtung für die medizinische Rehabilitation im Allgemeinen aber kein individuell durchsetzbarer Rechtsanspruch. In der Praxis ist in der PV dieses Problem nicht wirklich virulent geworden, zumal durch die sehr hohen Bewilligungsraten kein Anlass zu einer Klagsführung besteht. Ablehnungen in geringem Ausmaß bestehen nur dort, wo entweder eine Kontraindikation des Antragstellers oder ein derart geschwächter Gesundheitszustand vorliegt, der die Vornahme von Rehabilitationsmaßnahmen von zwei bis drei Stunden täglich nicht zulässt.

Instabile Patienten wären zunächst zur Remobilisation mit Zuständigkeit der Länder oder zu einem Genesungs- oder Erholungsaufenthalt durch einen KV-Träger einzuweisen.

\subsubsection{Rehabilitationsleistungen der Krankenversicherung}

Mit der 50. ASVG-Novelle hat der Gesetzgeber die medizinische Rehabilitation auch in der KV (Aufrechterhaltung der Selbstständigkeit der Versicherten) als Pflichtaufgabe eingeführt. In den EB zur RV dieser Gesetzesänderung wurde beabsichtigt, die medizinische Rehabilitation in der KV ab 1994 von einer Pflichtaufgabe zu einer Pflichtleistung umzugestalten. Es ist aber bis heute nur bei dieser Absichtserklärung geblieben.

Aufgrund der gegenwärtigen schlechten finanziellen Lage vieler KV-Träger werden medizinische Rehabilitationsleistungen für Alterspensionisten bzw Angehörige oft nicht erbracht und die PV-Träger aufgefordert, den Versicherten und Pensionisten Maßnahmen der Gesundheitsvorsorge zu gewähren. Damit ist eine Angehörigenrehabilitation neuerlich nicht ausreichend gewährleistet.

Von rechtlicher Relevanz sind die Urteile des OGH vom 18.2.2003, 10 ObS 258/02t und vom 23. 5. 2005, 10 ObS 7/05 k.

In diesen Erkenntnissen spricht der OGH aus, dass jedenfalls bei medizinischen Maßnahmen der Rehabilitation in der KV ein Anspruch des Versicherten auf gesetzmäßige Ermessensausübung besteht, der auch verfahrensmäBig nachprüfbar sein muss. Damit bejaht der OGH einen Anspruch auf Bescheiderlassung und nachfolgende Überprüfung der Ermessensentscheidung durch das Arbeits- und Sozialgericht. 
1.4.4 Rehabilitations- und Gesundheitsvorsorgerichtlinien des Hauptverbandes

Beachtlich sind auch die Maßnahmen der beruflichen und sozialen Rehabilitation. Zwecks einheitlicher Vorgangsweise der SV-Träger gibt es die RI für die Erbringung von Leistungen im Rahmen der Rehabilitation sowie von Leistungen im Rahmen der Festigung der Gesundheit und Gesundheitsvorsorge (RRK) des HV vom 1.9. 2000, wiederverlautbart als RRK 2005 im Internet (www.avsv.at). Neben der Festlegung der Maßnahmen der medizinischen Rehabilitation werden die beruflichen Maßnahmen der Rehabilitation aus der PV und die sozialen Maßnahmen der Rehabilitation aus der PV und UV geregelt. Auch eine Früherfassung durch die KV wird berücksichtigt, die nur mit einer völligen Vernetzung mit den PV-Trägern funktionieren kann.

Die wichtigsten Maßnahmen dieser Richtlinien und die Zuständigkeit werden im folgenden Raster dargestellt:

\begin{tabular}{|l|l|}
\hline \multicolumn{2}{|c|}{ Rehabilitations-(Gesundheitsvorsorge)richtlinien des HV (RRK) } \\
\hline & - Genesungsaufenthalte (KV) \\
& Erholungsaufenthalte (KV) \\
- & Landaufenthalte (KV - bei Ablehnung durch \\
medizinische Maßnahmen & KV-Ermessensleistung der PV) \\
(KV und PV) & Kuraufenthalte (PV) \\
& Krankenanstalten, die vorwiegend der \\
& Rehabilitation dienen (PV, KV) \\
& Hilfsmittel und Behelfe (KV, PV) \\
\hline & Arbeitstraining, Ein-, Um- oder Nach- \\
& schulung, Lehr- oder Schulausbildung (PV) \\
berufliche Maßnahmen (PV oder & Hilfen zur Fortsetzung der Erwerbstätig- \\
BVA im Rahmen der erweiterten & keit, wie Adaptierungen oder Anschaf- \\
Heilbehandlung) & fungen von Arbeitsgeräten (PV) \\
& Hilfeleistungen zur Erlangung einer Ar- \\
& beitsstelle oder einer anderen Erwerbstätig- \\
& keit (PV) \\
\hline & Adaptierung von Wohnräumen-zinsen- \\
& freie Darlehen oder Kostenzuschuss nach \\
& B-KUVG (PV, UV) \\
& Kostenzuschuss für Lenkerbefugnis \\
& (PV, UV) \\
& Darlehen oder Kostenzuschuss nach \\
& B-KUVG zum Ankauf oder Adaptierung \\
& eines PKWs (Darlehenshöchstgrenze) \\
soziale Maßnahmen & (PV, UV) \\
(PV und UV) & Übernahme von Transportkosten zu oder \\
& vom Arbeitsplatz (PV, UV) \\
& Ergänzende Leistungen (zB Unterhalts- \\
& beitrag an Angehörige bei erheblicher finan- \\
& zieller Mehrbelastung wegen Ausbildung des \\
& Versicherten (PV, UV) \\
\hline & \\
&
\end{tabular}

Von Bedeutung sind auch Vereinbarungen der Pensions- und UV-Träger mit dem AMS zur Durchführung der beruflichen Rehabilitation und Kostenteilung ( $\$ 307 \mathrm{c} \mathrm{Z} 1$ ASVG)

\subsection{Grundsätze für die medizinische Rehabilitation}

\subsubsection{Stationäre oder ambulante Rehabilitation}

Primär hat sich die medizinische Rehabilitation als sogenanntes „Anschlussheilverfahren" nach größeren Operationen am Stütz- und Bewegungsapparat (zB Hüftgelenke) oder nach erfolgreicher Akutbehandlung von Herzerkrankungen, wie $\mathrm{zB}$ nach Infarkten, entwickelt. Weitere Indikationen kamen dann aufgrund des medizinischen Fortschritts hinzu. Im Wesentlichen sind dies neurologische Erkrankungen (zB Schlaganfälle), Stoffwechselerkrankungen (zB Diabetes mellitus II), pulmologische Erkrankungen ( $\mathrm{zB}$ chronisch obstruktive Lungenerkrankung COPD, unfall- und neurochirurgisch versorgte Patienten sowie die onkologische Nachsorge).

In der UV nach dem ASVG ist die medizinische Rehabilitation nicht expressis verbis angeführt. Eine teleologische Interpretation der Bestimmungen der $\$ \$ 189$ bis 191 und 194 ASVG ergibt aber den Einsatz von Mitteln der stationären medizinischen Rehabilitation, um den Zweck und das Ziel der Unfallbehandlung erreichen und absichern zu können. Rudimentär findet sich in \$194 ASVG eine Norm, dass der HV in den Richtlinien über die Zusammenarbeit der Träger der KV und UV Fachärzte mit der Entscheidung über die Unfallheilbehandlung in der Folge bei sich oder in Sonderkrankenanstalten betrauen kann.

In der UV nach dem BSVG ist in $\$ 148$ leg cit (Aufgaben der UV) die medizinische Rehabilitation sowohl als ausdrückliche Aufgabe dieses Versicherungszweigs für Versehrte als auch mit ihren Maßnahmen im Rahmen der Unfallheil behandlung genannt. Eine ähnliche Regelung findet sich in $\$ 87$ B-KUVG für die medizinische Rehabilitation bei Dienstunfällen Beamter. Ob die Maßnahmen der medizinischen Rehabilitation für Bauern oder Beamte stationär oder ambulant zu erbringen sind, lässt der Gesetzgeber offen.

In der KV und PV (siehe die $\$ 154 \mathrm{a}$ Abs 2 und 7 ASVG, 99a Abs 2 und 7 GSVG, 96a Abs 2 und 7 BSVG, sowie 302 Abs 1 und 4 ASVG, 160 Abs 1 und 4 GSVG, 152 Abs 1 und 4 BSVG, 65a Abs 1 und 5 B-KUVG) werden nach Auffassung des $\mathrm{HV}$ diese Bestimmungen so interpretiert, dass aus teleologischer Sicht alle Formen der medizinischen Rehabilitation vom Gesetzgeber beabsichtigt waren:

nämlich eine

- stationäre,

- ambulante,

- teilstationäre und

- tagesklinische

Rehabilitation.

Von wesentlicher Bedeutung ist aber der Wille des Gesetzgebers, die ambulante Rehabilitation an krankenanstaltenrechtliche Kriterien zu binden, um damit auch das medizinische Rehabilitationsziel bei dieser Form der Rehabilitation im Vergleich zu den Strukturqualitätskriterien bei der stationären Rehabilitati- 
on zu garantieren. Ambulatorien bedürfen nach österreichischem Krankenanstaltenrecht einer eigenen Bewilligung und sollten nicht wettbewerbsverzerrend unter außer Achtlassung von Mindeststandards tätig sein. So gibt es derzeit ein Pilotprojekt von sechs Ambulatorien mit einer Begleitstudie zur ambulanten kardiologischen Rehabilitation, das ab Mai 2010 von den PV-Trägern und dem HV evaluiert werden soll.

Wesentlich ist auch, dass die Bedingungen „Rehabilitationsfähigkeit, Rehabilitationsziel und Rehabilitationsplan“ bei der medizinischen Rehabilitation der Phase 2 sowohl bei der stationären, als auch bei der ambulanten Rehabilitation ident sein müssen.

\subsubsection{Rehabilitation vor Pension}

$\$ 361$ Abs 1 letzter Satz ASVG (ähnlich auch \$\$194 Z 2 lit a GSVG, 182 Z 3 lit a BSVG) bestimmen, dass ein Antrag auf eine Pension aus dem Versicherungsfall der geminderten Arbeitsfähigkeit (oder der Erwerbsunfähigkeit) auch als Antrag auf Leistungen der Rehabilitation gilt. Der Gesetzgeber unterscheidet hiebei nicht zwischen den Arten der medizinischen, beruflichen und sozialen Rehabilitation. Grundgedanke des Gesetzgebers des Strukturanpassungsgesetzes II (BGBl 1996/201) war der Versuch der Eindämmung der Zuwächse der Altersund Invaliditätspensionen, die er mit Wartezeitverschärfungen, Schul- und Studienzeitennachkaufsverteuerungen und Ausdehnung von Rehabilitationsmöglichkeiten erreichen wollte.

Das generelle Problem liegt allerdings darin, dass Antragsteller auf obgenannte Pensionsarten bereits kaum mehr den Willen zu einer Berufsveränderung mehr aufbringen, noch dazu, wo auch eine erfolgreiche Rehabilitation keinen Anspruch auf einen konkreten Arbeitsplatz bewirkt. Letztlich hat auch die Judikatur des OGH in Sozialrechtssachen dem Kläger eine Pension zugesprochen, wenn der PV-Träger erst im Verfahren vor dem Sozialgericht eine Rehabilitation anbietet ("Verspätung"). Gelingt die Wiedereingliederung in den Arbeitsprozes durch eine erfolgreiche Rehabilitation nicht, so erlangt der Pensionswerber nich durch eine erfolgreiche Rehabilitation nicht, so erlangt einkommensverlust durch die Differenz vom Übergangsgeld ( $\$ 306$ ASVG) zum Arbeitslosengeld (Not standshilfe, $\$ 6$ Abs 1 AlVG). Sollte dem zweifellos legitimen Anspruch eines Primats der Rehabilitation vor einer Pensionsleistung zum Durchbruch verholfen werden, so müssten dafür mehr und stärkere Anreize geschaffen werden:

- früherer Rehabilitationsbeginn,

- Sicherheit des früheren oder eines neuen Arbeitsplatzes,

- Entgeltsicherung bei erfolgreicher Rehabilitation

- realitätsgerechte Behindertenbeschäftigung,

- Zulässigkeit der Rehabilitation auch während des Sozialgerichtsverfahrens, sofern dem Rehabilitanden ein Übergangsgeld bis zur Erlangung eines konkreten Arbeitsplatzes gewährt wird.

\subsubsection{Grundsatz der Rehabilitationsfähigkeit}

Da in Österreich seit mehr als einem Jahrzehnt in den Krankenanstalten das LKF-System eingeführt wurde, hat sich die Belagsdauer im Einzelfall verringert, nicht aber die Spitalshäufigkeit. Kürzere Aufenthalte in den Akutkrankenanstalten haben dazu geführt, dass die Patienten meist nicht sofort rehabilitationsfähig sind, sondern einer Genesungsphase bedürfen, insb, wenn sie bereits in höherem Alter sind. Da die KV-Träger aufgrund ihrer Finanzlage die Erholungsaufenthurückgedrängt haben, Aufenthalte mit Remobilisation der Patienten noch nicht die Regel sind, werden die Versicherten oft aus den Akutkrankenanstalten in die Rehabilitationszentren transferiert. Dies bedeutet dann eine Verlängerung des „normalen“ Rehabilitationsaufenthaltes für die PV-Träger mit Zusatzkosten für den Bund. Medizinisch ist ein Patient nur dann rehabilitationsfähig, wenn er nach Expertenmeinung mindestens 2 - 3 Stunden pro Tag Maßnahmen der Rehabilitation bewältigen kann. Für jeden Versicherten wird ein individueller Rehabilitationsplan erstellt, der vom behandelnden Rehabilitationsteam immer wieder überprüft und bei Bedarf zur Erreichung des Rehabilitationszieles abgeändert werden kann. Flexibilität hat in der medizinischen Rehabilitation somit einen hohen Stellenwert, zumal davon der optimale Rehabilitationserfolg abhängt.

\section{Begriffliche Abgrenzung von anderen Maßnahmen der Rehabilitation \\ 2.1 Berufliche Maßnahmen der Rehabilitation}

2.1.1 Rehabilitationszweck in der Unfallversicherung

Die zentrale Bestimmung findet sich in $\$ 198$ ASVG, mit der berufliche Maßnahmen für Versicherte in der UV geregelt werden. Rehabilitationsziel ist den Versehrten in die Lage zu versetzen, entweder seinen bisherigen oder, wenn dies nicht mehr erreicht werden kann, einen neuen Beruf auszuüben. Beispielsweise zählt der Gesetzgeber die wichtigsten Maßnahmen der beruflichen Rehabilitation auf:

- Berufliche Ausbildung zur Wiedergewinnung oder Erhöhung der Erwerbsfähigkeit.

- Ausbildung für einen neuen Beruf, soweit dies dem Versehrten zumutbar ist und er in seinem bisherigen Beruf wesentlich beeinträchtigt wäre.

- Gewährung von Zuschüssen, Darlehen bzw sonstigen Hilfsmaßnahmen zur Bewerkstelligung der Fortsetzung der Erwerbstätigkeit des Versehrten.

- Hilfe zur Erlangung einer Arbeitsstelle oder einer anderen Erwerbsmöglichkeit. Für die Übergangszeit - maximal aber vier Jahre - kann der UV-Träger einen Zuschuss bis zum vollen Entgelt bezahlen. Ferner kann der Versicherungsträger dabei den Versehrten mit einem Zuschuss oder einem Darlehen zur Beschaffung einer Arbeitskleidung oder -ausrüstung unterstützen. Auch dem Dienstgeber des Versehrten, der eine Arbeitsstelle angenommen hat, in der er seine volle Leistungsfähigkeit erst nach Erreichen des Rehabilitationszieles wieder erlangen kann, kann der UV-Träger für diese Übergangszeit - längstens für vier Jahre - einen Zuschuss bei Bezahlung des betriebsüblichen Entgelts gewähren. 


\subsubsection{Rehabilitationszweck in der Pensionsversicherung}

$\$ 303$ ASVG verweist auf $\$ 198$ leg cit mit Ausnahme der Gewährung von Zuschüssen, Darlehen bzw sonstigen Hilfsmaßnahmen zur Ermöglichung der Fortsetzung der Erwerbstätigkeit. Damit soll eine Wiederherstellung der Erwerbsfähigkeit in einem anderen Beruf gewährleistet werden. Im gleichen Beruf scheint die Fortsetzung der Erwerbstätigkeit nicht mehr möglich zu sein, zumal der Kreis der Normadressaten der Rehabilitation in der PV sich de lege late nur auf erwerbstätige Versicherte und Invaliditäts(Berufs-, Erwerbsunfähigkeits-) pensionisten bezieht, die offenbar bereits aus dem bisherigen Arbeits(Erwerbs-) prozess ausgeschieden sind. Im Übrigen ist nach $\$ 305$ ASVG der Behinderte vom PV-Träger über das Rehabilitationsziel und die Möglichkeiten (Maßnahmen) der Rehabilitation nachweislich und verständlich zu informieren und mit ihm eine Beratung durchzuführen. Werden sohin Maßnahmen der Rehabilitation in Betracht gezogen, so trifft den Versicherten eine Mitwirkungspflicht. Zweck der Rehabilitationsmaßnahmen in der PV ist die primäre Vermeidung von Pensionen der geminderten Arbeits(Erwerbsun-)fähigkeit, aber auch die sekundäre Vermeidung von Pflegegeldleistungen, die auch teilweise mit den genannten Pensionsleistungen anfallen können.

\subsubsection{Begriffliche Abgrenzung der beruflichen Rehabilitation zur} medizinischen Rehabilitation

Die medizinische Rehabilitation schafft die Voraussetzungen für die Ermöglichung einer beruflichen Rehabilitation. Nach Behandlungen mit dem jeweils neuesten Stand der Medizin - in der UV: wenn und solange dies die Behandlung mit allen geeigneten Mitteln erfordert - soll der Rehabilitand nach einer ausreichenden Genesungs- und Erholungsphase in die Lage versetzt werden, entweder beruflich einen Vollerwerb in seinem herkömmlichen Beruf wieder anzustreben oder sich in einem neuen Beruf ausbilden zu lassen. Der Gesetzgeber geht derzeit prinzipiell von einer Normalarbeitszeit je nach Branche von $36-40$ Stunden aus. Eine Rehabilitation auf einen Beruf mit Teilzeitarbeit und Teilpension(-rente) kennt der Gesetzgeber nicht. Sohin ist der Angelpunkt für den Erfolg einer beruflichen Rehabilitation die medizinische Heilbehandlung und ihr optimales Ergebnis.

Zusammenfassend ist festzustellen, dass die medizinische Rehabilitation der primäre Faktor für das weitere Rehabilitationsgeschehen ist und bei Erfolg die geeigneten physischen und psychischen Rahmenbedingungen für das Instrumentarium der beruflichen Rehabilitation schafft (siehe auch $\$ \$ 14-16$ und 22 der Rehabilitationsrichtlinien des HV [RRK 2005]).

\subsection{Soziale Maßnahmen der Rehabilitation}

2.2.1 Soziale Maßnahmen der Rehabilitation in der Unfallversicherung

Wiederum findet sich die ursprüngliche Bestimmung über soziale Maßnahmen der Rehabilitation im Recht der UV ( $\$ 201$ ASVG). Die Legaldefinition des $\$ 201$ ASVG bezeichnet jene Leistungen als solche der sozialen Rehabilitation, die außer der Unfallheilbehandlung, die auch die medizinische Rehabilitation inkludiert und den beruflichen Maßnahmen der Rehabilitation zusätzlich geeignet sind, das in $\$ 172$ Abs 2 ASVG angestrebte Rehabilitationsziel zu erreichen. Dieses umfasst jene Maßnahmen zur Ergänzung der medizinischen und beruflichen Rehabilitation, die geeignet sind, Versehrte bis zu einem solchen Grad ihrer Leistungsfähigkeit wiederherzustellen, der sie in die Lage versetzt, im beruflichen und wirtschaftlichen Leben und in der Gemeinschaft einen ihnen angemessenen Platz möglichst dauernd einnehmen zu können.

Der Gesetzgeber zählt im $\$ 201$ Abs 2 ASVG - unter Bedachtnahme auf die wirtschaftlichen Verhältnisse des Versehrten - die wesentlichsten Maßnahmen auf, die auch durch die Richtlinien des HV zur Vereinheitlichung der Vollziehung (RRK 2005, $\$ 17-21$ ) präzisiert wurden:

- Gewährung eines Zuschusses bzw eines Darlehens (oder beides) zur Adap-

tierung einer Wohnung oder Teilen davon (zB Behindertentoilette)

- Zuschuss zur Erlangung einer Lenkerberechtigung,

- Zuschuss oder Darlehen (oder beides) zum Ankauf bzw zur Adaptierung eines behindertengerechten PKWs.

Letztere beide Maßnahmen werden nur jenen Versehrten gewährt, denen aufgrund ihrer Behinderung die Benützung eines öffentlichen Verkehrsmittels nicht zumutbar ist.

In $\$ 201$ Abs 3 ASVG wird eine weitere Maßnahme der sozialen Rehabilitation, nämlich die Förderung des Versehrtensports, genannt, wenn folgende Voraussetzungen erfüllt werden:

- Mannschaftssport (Gruppen),

- ärztliche Betreuung muss vorhanden sein

- Zuschüsse an die entsprechenden Institutionen,

- Nachweis der widmungsgemäßen Verwendung.

Die Normen des $\$ 201$ Abs 4 erlauben noch weitere Förderungen an taxativ aufgezählte Einrichtungen:

- einer Gemeinde,

- einer Gebietskörperschaft (offenbar Bund oder ein Bundesland)

- des AMS

- des BSB,

- eines SV-Trägers,

- der Arbeiterkammer und Wirtschaftskammer, wenn sie die Beschäftigung des Versehrten in einem integrativen Betrieb und in einer Einrichtung der Beschäftigungstherapie fördern.

Schließlich gestattet der Gesetzgeber ( $\$ 201$ Abs 5 ASVG) den Einsatz von Finanzmitteln der UV zur Förderung und Unterstützung gemeinnütziger Einrichtungen (Institutionen ohne Gewinnabsicht, daher keine Gewerbebetriebe) die die Förderung der wirtschaftlichen, sozialen und kulturellen Interessen von Behinderten zum Ziel haben.

\subsubsection{Soziale Maßnahmen der Rehabilitation in der}

\section{Pensionsversicherung}

Die Regelung über soziale Maßnahmen der Rehabilitation in der PV ist jener in der UV nachgebildet. $\$ 304$ Abs 1 ASVG bestimmt, dass sie jene Leis- 
tungen umfassen, die über die medizinischen und beruflichen Maßnahmen der Rehabilitation hinaus geeignet sind, zur Erreichung des im $\$ 300$ Abs 3 ASVG Rehabilitation hinaus geeignet sind, ckungsgleich mit jenem der UV in $\$ 172$ Abs 2 ASVG und bedeutet wiederum, dass der Rehabilitand außer der medizinischen Wiederherstellung oder Besserung seines Gesundheitszustandes sowie beruflicher Maßnahmen der Rehabilitation zusätzlich unterstützt werden soll, im beruflichen und wirtschaftlichen Leben sowie in der Gemeinschaft einen ihm angemessenen Platz möglichst dauernd einnehmen zu können.

$\$ 304$ Abs 2 ASVG verweist für Maßnahmen der sozialen Rehabilitation in der PV auf die Bestimmungen des $\$ 201$ Abs 2 bis 4 in der UV, schließt jedoch die Gewährung von Zuschüssen zur Wohnungsadaptierung sowie zum Ankauf oder der Adaptierung eines PKWs aus.

Letztlich können auch Finanzmittel der PV - ähnlich wie in der UV - zur Förderung und Unterstützung von gemeinnützigen Einrichtungen verwendet werden, die die Förderung der wirtschaftlichen, sozialen und kulturellen Interessen von Sozialversicherten zum Ziel haben ( $\$ 304$ Abs 3 ASVG). Der Gesetzgeber hat aber diese Gelder mit 0,005 vT der Erträge an PV-Beiträgen limitiert.

Die sozialen Maßnahmen der Rehabilitation in der PV sollen sowohl Versicherte als auch Invaliditätspensionisten im Arbeitsprozess und im gesellschaftlichen Leben erhalten, sodass die Regelung des Ausschlusses von Zuschüssen für die Wohnungsadaptierung oder eines Erwerbs eines behindertengerechten PKWs bzw dessen Adaptierung obsolet erscheint.

\subsubsection{Soziale Krankenversicherung}

In der KV sind nur Maßnahmen der medizinischen Rehabilitation vorgesehen. $\$ 154$ a ASVG ist im Wesentlichen $\$ 302$ ASVG nachgebildet. Auf die materielle Abgrenzung zur Krankenbehandlung wird später noch eingegangen. Das wesentliche Rehabilitationsziel in der KV ist der Erhalt der Selbstständigkeit der Rehabilitanden im täglichen Leben.

\subsection{Besonderheiten der beruflichen und sozialen Rehabilitation in den Sondergesetzen}

Im B-KUVG werden Maßnahmen der erweiterten Rehabilitation $(\$ 70 \mathrm{iVm}$ $\$ 70 \mathrm{~b}$ ) vom Gesetzgeber zugelassen, die im Prinzip auch Maßnahmen der beruflichen und sozialen Rehabilitation vorsehen. Sie sind im Wesentlichen mit jenen der UV und PV im ASVG ident ( $\$ 87$ Abs 2 iVm $\$ \$ 99 a-c$ B-KUVG). Ausgeschlossen sind davon aber:

- Ruhe- und Versorgungsgenussbezieher

- Ruhe- und Versorgungsgenussbezieher, die Politiker oder Mitglieder des

VfGH waren,

Pensionsbezieher nach der Salinenarbeiter-Pensionsordnung 1967, BGBl $1968 / 5$.
Im GSVG regelt $\$ 161$ die beruflichen Maßnahmen der Rehabilitation in der PV. Die berufliche Ausbildung wird unbefristet gewährt, solange durch sie die Erreichung des Rehabilitationszieles zu erwarten ist. Ferner ist eine Zuschussgewährung zur Ermöglichung der Fortsetzung der Erwerbstätigkeit nicht vorgesen. Von Bedeutung ist, dass die Rehabilitation in eine andere selbstständige Erwerbstätigkeit einer spezifischen Rehabilitationsplanung bedarf. In der Praxis kommt sie selten vor (zB Erwerb einer Trafik).

Bei der sozialen Rehabilitation in der PV wurde $\$ 162$ GSVG der Bestimmung des $\$ 201$ ASVG in der UV mit Ausnahme von Zuschüssen zur Wohnungsadaptierung oder zum Ankauf oder der Adaptierung eines PKWs nachgebildet.

Das BSVG orientiert sich in der UV in $\$ 148 \mathrm{y}$ bei den Maßnahmen der beruflichen Rehabilitation primär am ASVG $(\$ 198)$ stellt aber in $\$ 148 y$ Abs 2 Z zusätzlich auf die Beistellung von Ersatzarbeitskräften zur Kompensation des Arbeitsausfalles des Versehrten ab. Primäres Rehabilitationsziel ist: „Der Bauer soll Bauer bleiben".

Bei der sozialen Rehabilitation wurden ebenfalls die Bestimmungen des $\$ 149$ a BSVG kongruent mit jenen der UV nach dem ASVG gestaltet. Finanzmittel der bäuerlichen UV dürfen ebenfalls zur Förderung von gemeinnützigen Einrichtungen verwendet werden.

\section{Strukturen und Zuständigkeiten}

\subsection{Die Leistungen der medizinischen Rehabilitation im Überblick} bei der Unfallversicherung

Grundlage für die Leistungen in der UV ist primär das ASVG. Der Geltungsbereich dieses Gesetzes erstreckt sich im Wesentlichen auf sämtliche Dienstnehmer, Selbstständige als Gewerbetreibende, sogenannte „neue Selbstständige“, wie zB Werknehmer, Schüler und Studenten. Die UV der Bauern ist im BSVG geregelt und hatte stets eigenständigen Charakter, zumal die historischen Wurzeln auf das Landarbeitsgesetz 1929 zurückgingen. Auch die Notare hatten seit 1926 ein eigenes Leistungsrecht mit dem NVG, zuletzt NVG 1972, in dem sowohl die PV als auch die UV der Notare und Notariatskandidaten geregelt ist.

\subsubsection{Die medizinische Rehabilitation und der Eintritt des}

\section{Versicherungsfalls}

Der bereits früher erwähnte $\$ 172$ Abs 2 ASVG als Legaldefinition der Rehabilitation in der UV stellt auf die medizinischen, beruflichen und sozialen Maßnahmen der Rehabilitation ab, wenn der Versicherungsfall in der UV nach $\$ 174$ ASVG eingetreten ist:

- Unfallereignis bei Arbeitsunfällen (oder diesen gleichgestellten Unfällen),

- Beginn der Berufskrankheit oder Beginn der Minderung der Erwerbsfähigkeit, wenn dies für den Versicherten günstiger ist.

$\$ 189$ Abs 1 ASVG definiert, dass die Unfallheilbehandlung mit allen geeigneten Mitteln zu besorgen ist, um den Unfallschaden zu beseitigen oder zumindest zu bessern und eine Verschlimmerung zu verhüten. Damit ist aber auch die 
Leistung der medizinischen Rehabilitation inkludiert, obwohl sie nicht expressis verbis im Abschnitt III, 1. Unterabschnitt des ASVG erwähnt ist.

\subsubsection{Parallelleistungen der medizinischen Rehabilitation zur}

\section{Unfallheilbehandlung}

$\$ 189$ Abs 2 ASVG zählt demonstrativ fünf Kategorien von Leistungen mit medizinischem Charakter auf und ermächtigt dazu die Satzung des UV-Trägers unter Bedachtnahme auf die wirtschaftlichen Verhältnisse des Versicherten, Reise- und Transportkosten hiefür zu übernehmen. Die sechs Leistungsarten lauten daher:

- ärztliche Hilfe,

- Heilmittel,

- Heilbehelfe,

- Pflege in Kranken-, Kur- und sonstigen Anstalten,

- Hilfsmittel,

- Reise- und Transportkosten.

Diese sechs Kategorien von Leistungen anlässlich einer Akutbehandlung benötigt in der Regel auch der Rehabilitand, allerdings bereits in einer fortge schrittenen Heilungsphase. Die Besonderheit in der UV ist die Gewährung der Unfallheilbehandlung ohne zeitliche Beschränkung, so lange und so oft eine Besserung der Unfallfolgen oder der Berufskrankheit bzw eine Steigerung der Erwerbsfähigkeit zu erwarten ist bzw eine Verschlimmerung verhütet werden kann ( $\$ 190$ ASVG). Dies bedeutet, dass auch die medizinische Rehabilitation in der UV unbegrenzt zu gewähren ist, so lange eine Besserung des Gesundheitszustandes und der Erwerbsfähigkeit möglich sind.

3.1.3 Leistungserbringer und -träger der medizinischen Rehabilitation in der Unfallversicherung

\section{Leistungserbringer:}

Die Leistungen der Unfallheilbehandlung werden in eigenen Unfallspitälern der AUVA oder in Unfallabteilungen von öffentlich rechtlichen oder privaten Krankenanstalten erbracht. Bei geringeren körperlichen Beeinträchtigungen erfolgen Leistungen in Unfallambulanzen der Spitäler. Auch eine ambulante Versorgung der Unfallopfer durch niedergelassene freiberufliche Ärzte sowie in Ambulatorien ist rechtlich zulässig.

\section{Leistungsträger sind in Österreich:}

- AUVA: zuständig in Wesentlichen für DN ( $\$ 4$ Abs 1 und 4 ASVG), gewerbliche und neue Selbstständige ( $\$ 8 \mathrm{Abs} 1 \mathrm{Z} 3$ lit a ASVG), Schüler und Studenten ( $\$ 8$ Abs $1 \mathrm{Z} 3$ lit h und $\mathrm{i} A S V G$ ).

- Versicherungsanstalt für Eisenbahnen und Bergbau: zuständig für Bedienstete von Schienen- und Seilbahnen bzw Bergbaubeschäftigte ( $\$ 472 \mathrm{~b}$ Z 2 ASVG).

- Versicherungsanstalt öffentlich Bediensteter: zuständig im Wesentlichen für Beamte (öffentlich rechtliches Dienstverhältnis, sofern nicht auch ein
Dienstverhältnis nach dem ASVG gegeben ist), unkündbare Dienstnehmer dieser Versicherungsanstalt, Ruhe- und Versorgungsgenussbezieher (pensionierte Beamte und deren Witwer/Witwen), Politiker, wie $\mathrm{zB}$ Abgeordnete zum Nationalrat, zu den Landtagen, zum Bundesrat, Mitglieder der Bundes- und Landesregierungen, der Bundespräsident, Mitglieder des Verfassungsgerichtshofs ( $\$ 1 \mathrm{~B}-\mathrm{KUVG}$ ).

- SVB: zuständig im Wesentlichen für land- und forstwirtschaftliche Betriebsführer, in deren Betrieb tätige Kinder, Enkel, Wahl-, Stief- und Schwiegerkinder, Eltern, Geschwister, wenn diese Personen aufgrund ihrer Beschäftigung nicht einer Pflichtversicherung nach dem ASVG oder GSVG unterliegen $(\$ 3 \mathrm{BSVG})$.

\subsection{Die Leistungen der medizinischen Rehabilitation}

im Überblick bei der Pensionsversicherung

Die Leistungen der medizinischen Rehabilitation in der PV sind in $\$ 302$ ASVG und den früher bereits erwähnten Parallelbestimmungen in den Sondergesetzen für die Selbstständigen, Bauern und Beamten geregelt. $\$ 302$ ASVG - obwohl anders als die Bestimmung des $\$ 189$ formuliert - hat aber den gleichen Inhalt wie diese Norm. $\$ 300$ Abs 3 ASVG hat auch die wörtlich idente Formulierung wie $\$ 172$ Abs 2 ASVG in der UV. Allerdings ist in der PV die medizinische Rehabilitation nicht perpetuiert. Aufgrund medizinischer Erfahrungen der chefärztlichen Dienste der PV-Träger und anderer externer Rehabilitationsexperten werden in der Regel Rehabilitationsaufenthalte mit drei Wochen (zB bei orthopädischer Rehabilitation) oder vier Wochen ( $\mathrm{zB}$ bei neurologischer Rehabilitation) befristet.

Während bei der UV die stationären Rehabilitationsaufenthalte meist in eigenen Rehabilitationszentren stattfinden, hat die PV für $55 \%$ der Einweisungen eigene Einrichtungen, während $\mathrm{zu} 41 \%$ diese in private Rehabilitationszentren und $\mathrm{zu} 4 \%$ in ausländische Einrichtungen (meist Deutschland) erfolgen (Stand 2007). Pilotprojekte mit geringen Fallzahlen gibt es hinsichtlich der kardiologischen und orthopädischen Rehabilitation. Ein Nachsorgezentrum in Wien, 2. Bezirk, betreibt die PVA bei ihrer Hauptstelle selbst.

\subsubsection{Die Leistungserbringer der medizinischen Rehabilitation in der} Pensionsversicherung

Die Leistungen werden indikationsbezogen in der Regel von Sonderkrankenanstalten, die der medizinischen Rehabilitation dienen, erbracht. Ambulante Rehabilitationsleistungen werden derzeit nur in sehr geringem Ausmaß mit wenigen Pilotprojekten geleistet. Es ist dabei manchmal auch die Strukturqualität bei diesen ambulanten Betreibern fraglich. Einige sind aber auch sehr ambitioniert. Die Rehabilitationsprogramme und -ziele sind prinzipiell auf die gesundheitliche Verbesserung zwecks Erhalt der Berufsfähigkeit und der Vermeidung von Pflegebedürftigkeit abgestellt und müssen qualitätsgesichert und ausreichend dokumentiert sein. 


\subsubsection{Leistungsträger der medizinischen Rehabilitation in der}

\section{Pensionsversicherung}

In der Folge werden die vier maßgeblichen PV-Träger der SV erwähnt.

\section{- PVA}

Dieser Träger ist für $85 \%$ der erwerbstätigen Pensionsversicherten (sämtliche DN, zB Arbeiter, Angestellte [ $\$ 4$ Abs 1 ASVG], freie DN [ $\$ 4$ Abs 4 ASVG], im ASVG freiwillig selbst- und weiterversicherte Personen [ $\$ \$ 16 \mathrm{a}$, $17,18,18 \mathrm{a}$ und b ASVG]) und alle Invaliditäts- bzw Berufsunfähigkeitspensionsbezieher nach dem ASVG, ausgenommen Eisenbahn- und Bergbaubedienstete, zuständig.

- Versicherungsanstalt für Eisenbahnen und Bergbau

Zuständig für erwerbstätige Versicherte, die bei Schienen- und Seilbahnen, sowie im Bergbau beschäftigt sind. Weiters zuständig für die betreffenden Invaliditäts-, Berufsunfähigkeits-, und Dienstunfähigkeitspensionsbezieher.

- SVA

Zuständig für alle gewerblichen und neuen Selbstständigen, Freiberufler, wie niedergelassene Ärzte, selbstständige Apotheker und Patentanwälte, die in der PV pflichtversichert sind, sowie Bezieher einer Erwerbsunfähigkeitspension nach dem GSVG.

- SVB

Zuständig für alle land- und forstwirtschaftlichen Betriebsführer, hauptberuflich im Betrieb beschäftigten Ehegatten, Kinder, Enkel-, Wahl-, Stief- und Schwiegerkinder, Übergeber, die hauptberuflich im Betrieb beschäftigt sind, Betriebsführung von Ehegatten auf gemeinsame Rechnung und Gefahr oder hauptberufliche Beschäftigung des einen Ehegatten im Betrieb des anderen sowie Bezieher einer Erwerbsunfähigkeitspension nach dem BSVG.

\subsection{Die Leistungen der medizinischen Rehabilitation}

in der Krankenversicherung

Sie sind grundsätzlich in $\$ 154$ a ASVG geregelt und wie jene in $\$ 302$ ASVG gestaltet. Lediglich in $\$ 154 \mathrm{a}$ Abs $2 \mathrm{Z} 2$, der $\$ 302$ Abs 1 Z 2 ASVG entspricht, fehlt der Hinweis auf $\$ 202$ ASVG, welcher die Anpassung und den persönlichen Gebrauch der Hilfsmittel festlegt. Auch das Rehabilitationsziel in der KV ist ein anderes:

- Durchführung im Anschluss an die Krankenbehandlung,

- Sicherung des Erfolges der Krankenbehandlung,

- Erleichterung der Folgen der Krankheit.

Damit ist auch indirekt die Vermeidung oder Hinausschiebung von Pflegebedürftigkeit verbunden.

\subsubsection{Leistungserbringer der medizinischen Rehabilitation in der}

\section{Krankenversicherung}

Primär sind wiederum jene öffentlich rechtlichen oder privaten Anbieter von Gesundheitsdienstleistungen, wie in der UV und PV maßgeblich. Im Ge- gensatz zur UV ist aber die Krankenbehandlung durch das Gesetz ( $\$ 133$ Abs 2 ASVG) auf eine ausreichende und zweckmäßige, das Maß des Notwendigen nicht zu überschreitende Vorgangsweise eingeschränkt. Damit könnte die Auf fassung vertreten werden, dass auch bei der medizinischen Rehabilitation nach der Krankenbehandlung (meist nach Akutbehandlung im Spital), der Rahmen der Krankenbehandlung nicht überschritten werden darf. Eine solche Einschränkung besteht zweifellos bei der medizinischen Rehabilitation in der PV und UV nicht. Da aber die medizinische Rehabilitation in der KV ( $\$ 116$ Abs 1 Z 4 154a) eine eigene Leistung ist und das Rehabilitationsziel die Selbstständigkeit („ohne Betreuung und Hilfe") des Rehabilitanden ist, spricht dies eher für eine Gleichbehandlung wie in der UV und PV.

Der Gesetzgeber sieht weiters in $\$ 154$ a vor, dass der KV-Träger die Durchführung von Maßnahmen der medizinischen Rehabilitation an einen PV-Träger mit dessen Zustimmung gegen Kostenersatz übertragen darf. Weiters haben die KV-Träger die von ihnen zutreffenden medizinischen Rehabilitationsmaßnahmen mit anderen in Betracht kommenden Versicherungsträgern abzustimmen. Insb sind koordinierte Vereinbarungen mit den PV-Trägern damit gemeint (vgl $\$ 307 \mathrm{c}$ ASVG), zumal das Rehabilitationsziel der KV nicht das Arbeitsmarktservice berührt. Im Gegensatz zur UV sind wie bei der PV Maßnahmen der Gesundheitsvorsorge (zB Kur) und der Festigung der Gesundheit (zB Erholungsaufenthalt, siehe $\$ \$ 307 \mathrm{~d}$ und 155) aber ausgenommen. Von besonderer Bedeutung ist aber die Bestimmung des $\$ 302$ Abs 2 ASVG, nach der der PV-Träger die Gewährung von Maßnahmen der medizinischen Rehabilitation eines KV-Trägers jederzeit an sich ziehen kann. Es ist dies eine Übernahme ex lege, zumal der Gesetzgeber den Zusammenhang der Rehabilitationsmaß nahmen der KV verlangt. Der Anspruch des Versicherten gegenüber dem KVTräger geht in diesem Fall voll auf den PV-Träger über. Die Selbstverwaltung der PV vertritt eine restriktive Meinung, die zu einer Novellierung Anlass gab. Trotz einiger Bemühungen kam es noch nicht zu einer Regierungsvorlage oder einem Gesetzesbeschluss.

\subsubsection{Leistungsträger in der medizinischen Rehabilitation der}

\section{Krankenversicherung}

Sie haben nur wenige eigene Einrichtungen und treten meist nur subsidiär in Erscheinung, zumal die Finanzmittel insb bei einigen Gebietskrankenkassen sehr knapp geworden sind.

- 9 Gebietskrankenkassen,

- 6 Betriebskrankenkassen,

- SVA,

- SVB,

- Versicherungsanstalt öffentlich Bediensteter,

- Versicherungsanstalt für Eisenbahnen und Bergbau,

sind für nicht erwerbstätige Versicherte, Alters- und Hinterbliebenenpensionsbezieher (ausgenommen: Invaliditäts-, Berufs-, Dienst-, Erwerbsunfähigkeitspensionsbezieher) und Angehörige zuständig. 


\section{Materielle Abgrenzung zu anderen Leistungsbereichen des Versorgungssystems}

4.1 Medizinische Rehabilitation versus Krankenbehandlung

Ist die medizinische Rehabilitation eine Annexleistung zur Krankenbehandlung oder ist sie eine Leistung sui generis?

$\$ 154$ a ASVG und Parallelbestimmungen stellen auf eine Gewährung der medizinischen Rehabilitation als Leistung nach pflichtgemäßem Ermessen ab. Dies bedingt zwar keinen subjektiven Rechtsanspruch, sondern die objektive Möglichkeit zur Verfügungstellung der Leistung, die als Pflichtaufgabe bezeichnet wird. Der OGH (siehe zB 23. 5. 2005, 10 ObS 7/05k = SSV-NF 19/34) hat aber den Rechtsweg zur Nachprüfung des ausgeübten Ermessens für zulässig erklärt. Der Versicherte hat einen Anspruch auf die fehlerfreie Ausübung des dem Versicherungsträgers eingeräumten Ermessens, nämlich auf ein ordnungsgemäßes Verfahren mit Sachverhaltsfeststellung und Offenlegung der maßgeblichen Tatsachengrundlagen, die auch finanzieller Natur sein können.

Die Krankenbehandlung nach $\$ 133$ ASVG ist hingegen eine Pflichtleistung mit Rechtsanspruch, wenn die Tatbestandsmerkmale des $\$ 133$ Abs 2 erfüllt werden. Die Rechtsprechung des OGH hat in jüngerer Zeit (18. 2. 2003, 10 ObS 258/02) die Trennlinie zur Asylierung (= Pflegefall) dahingehend gezogen, dass immer noch eine Möglichkeit ärztlicher Einflussnahme iS einer Heilung, Besserung oder Verhütung von Verschlimmerungen existiert.

Ein Erlass des BMAS vom 20. 1. 1992, Zl 26.060/2-5/91 vertritt ebenfalls die Auffassung, dass die medizinische Rehabilitation in der KV eine eigene Leistung ist, zumal sie im Anschluss an die Krankenbehandlung erfolgt und ein eigenes Ziel verfolgt, das über jenes der Krankenbehandlung hinausgeht. Der Erlass sieht für den Rehabilitanden ua zwei Besserstellungen gegenüber der Krankenbehandlung vor:

- keine Obergrenze für Hilfsmittelkosten ( $\$ 154 \mathrm{ASVG}$ ),

- keine Rezeptgebühr.

Im Übrigen hat auch der Gesetzgeber des Strukturanpassungsgesetzes 1996 bei der Einführung von Zuzahlungen bei Rehabilitations- und Kuraufenthalten für die medizinische Rehabilitation generell nur die Stufe 1 (2009: $€ 7,-$ pro Verpflegstag bis maximal 28 Tage im Kalenderjahr) vorgesehen.

\subsection{Abgrenzung zur Anstaltspflege bei Akuterkrankung}

Das Gesetz ( $\$ 144$ ASVG) kennt keine Legaldefinition der Anstaltspflege. Es sieht vier Tatbestände vor, bei deren Erfüllung der Erkrankte sich einer Anstaltspflege unterziehen muss:

- Art der Krankheit, die häusliche Pflege nicht zulässt,

- Verhalten oder Zustand erfordert eine fortgesetzte Beobachtung,

- wiederholte Zuwiderhandlung gegen Normen der Krankenordnung,

- bei ansteckender Krankheit.

Eine medizinische Rehabilitation kommt naturgemäß erst nach erfolgreicher Anstaltspflege und Remobilisation in Betracht. Der betroffene Patient soll auch rehabilitationsfähig sein. Schließlich müssen die Leidenszustände auch besserbar sein. Eine heikle Schnittstelle bildet die Asylierung. Darunter versteht man den Übergang zum Pflegefall, wenn keinerlei Besserungsmöglichkeit meh besteht. Damit wäre auch die medizinische Rehabilitation nur mehr zur Stabilisierung des Gesundheitszustandes zulässig, wobei aber bei fortschreitendem körperlichen und geistigen Verfall kaum mehr ein Rehabilitationsziel in Betracht kommen wird.

\subsection{Abgrenzung zur medizinischen Hauskrankenpflege}

Die medizinische Hauskrankenpflege ( $\$ 151$ ASVG und Parallelbestimmungen) ist eine krankenhausersetzende Leistung (vgl OGH 22. 5. 2001, 10 ObS 315/00), die befristet gewährt wird. Grundsätzlich sieht der Gesetzgeber hiefür bis zu vier Wochen vor, danach kann eine Verlängerung nur durch die chef- und kontrollärztlichen Dienste der SV-Träger gewährt werden. Sie wird durch Angehörige des gehobenen Dienstes für Gesundheits- und Krankenpflege erbracht die vom KV-Träger zur Verfügung gestellt werden oder mit dem KV-Träger einen Vertrag haben bzw im Rahmen von Vertragseinrichtungen tätig sind, die medizinische Hauskrankenpflege betreiben.

Die Tätigkeit hiezu erfolgt nur auf ärztliche Anordnung. Sie umfasst medizinische Leistungen und qualifizierte Pflegeleistungen. Die Grundpflege und hauswirtschaftliche Versorgung sind ausgeschlossen und keine Leistungen der KV. Führt die Hauskrankenpflege zu einem gesundheitlichen Erfolg, wäre eine medizinische Rehabilitation zur Stabilisierung durchaus zulässig, wenn die Rehabilitationsfähigkeit gegeben ist. Vermag auch die Hauskrankenpflege keinen Heilungserfolg oder zumindest irgendeine Verbesserung des Gesundheitszustandes herbeizuführen, so wäre die medizinische Rehabilitation unzulässig.

\subsection{Medizinische Rehabilitation und die Gewährung} von Heilbehelfen und Hilfsmitteln

Die Maßnahmen der medizinischen Rehabilitation sollen den Rehabilitanden bestmöglich versorgen. Daher sollen ihm alle jene Heilbehelfe (zB Brillen) und Hilfsmittel (zB Rollstuhl) ohne finanzielle Belastung zur Verfügung stehen. Der Gesetzgeber geht damit von einer umfassenden Gesundheitssicherung aus, die auch eine erfolgreiche Krankenbehandlung oder Anstaltspflege sowohl aus finanziellen als auch humanitären Gründen absichern soll.

\subsection{Medizinische Rehabilitation und Pflegebedürftigkeît} nach $\$ \S 4$ und $4 a$ BPGG

Manchmal wurde die Auffassung vertreten, dass der Bezug von Pflegegeld insb einer höheren Stufe eine medizinische Rehabilitation nicht mehr zulässt. Diesem Argument kann aber entgegengehalten werden, dass auch bei Pflegegeldbeziehern in der Regel zusätzlich eine Krankheit auftreten kann, die durchaus bei erfolgreicher Krankenbehandlung eine Verbesserung des Gesundheitszustandes bewirken kann ( $\mathrm{zB}$ Schlaganfall und erfolgreiche Akutbehandlung). Gerade in diesen Fällen würde die medizinische Rehabilitation nicht nur menschliches Leid 
lindern helfen, sondern auch eine Höherreihung des Pflegegeldes verhindern oder zumindest hinausschieben.

\section{Rechtsprobleme und Systemkritik \\ 5.1 Generelle Defizite in der medizinischen Rehabilitation}

Da die Rehabilitation sich sehr unsystematisch entwickelt hat, gibt es daher sowohl legistisch als auch medizinisch Defizite, die sich aber nach und nach verringern. Dies wird vor allem durch die Weiterentwicklung der Rehabilitationsmedizin sowie einen entsprechenden Input durch die medizinischen Fachgesellschaften und SV-Träger bewirkt, indem eine Reihe von Pilotprojekten in Gang gesetzt wurde.

\section{Fehlen gesetzlicher Bestimmungen (zB ambulante Rehabilitation)}

Die gegenwärtigen Bestimmungen in den SV-Gesetzen bauen primär auf der Situation von 1960-1980 auf, wo ein starker Trend zur Errichtung von Rehabilitationszentren bestand. Die ab Mitte der 80er Jahre des 20. Jahrhunderts einsetzende budgetäre Knappheit der PV-Träger hat dazu geführt, dass bestenfalls Rehabilitationszentren adaptiert, aber kaum mehr neu gebaut wurden. Ausgehend von der Entwicklung in der BRD, wo auch durch die wirtschaftliche Entwicklung viele Rehabilitationskliniken nur zu Grenzkosten oder Verlusten arbeiteten und daher schließen mussten und der Verringerung der öffentlichen Budgetmittel wurde eine ,ambulante Rehabilitation“ nun stärker in Betracht gezogen.

Neben den medizinischen Voraussetzungen muss der Rehabilitand über die zur Inanspruchnahme der Rehabilitation erforderliche Mobilität verfügen. AuBerdem muss auch die häusliche Versorgung sichergestellt sein (Bundesarbeitsgemeinschaft für Rehabilitation, BRD, 2000).

Die ambulante Rehabilitation bedarf bei einer legistischen Lösung einer genauen Analyse und einiger klarer Legaldefinitionen:

- Klarstellung der Wohnortnähe,

- Definition von Mindeststandards der Einrichtung (zwecks gesicherter Qua-

litätskontrolle wäre eine krankenanstaltenrechtliche Basis anzustreben),

- Verkürzung stationärer Rehabilitationsaufenthalte mit additiver ambulanter Nachsorge,

- krankenhausersetzende Rehabilitation und ihre Ausprägung,

- Lösung bei unterschiedlicher Therapiedichte (Stadt/Land),

- einheitliche und gesicherte Dokumentation über das (teilweise) Erreichen oder Nichterreichen des Rehabilitationszieles,

- Unterstützung von Selbsthilfepotentialen.

\section{Ausbau einer flächendeckenden medizinischen Versorgung}

\section{(zB psychiatrische Rehabilitation)}

Der HV hat sich bereits mit dem Rehabilitationskonzept 1999 bemüht, strukturelle Defizite in der medizinischen rehabilitativen Versorgung bei bestimmten Indikationen, wie zB der neurologischen Rehabilitation, abzubauen.
Im Rehabilitationsplan 2004 sind Richtlinien für Strukturqualitätskriterien auch für die psychiatrische Rehabilitation enthalten. Es ist seit damals der SV auch gelungen, mit mehreren privaten Einrichtungen für die psychiatrische Rehabilitation Verträge zu schließen oder in Aussicht zu nehmen. Der Rehabilitationsplan 2009 sieht einen weitgehenden flächendeckenden Ausbau für diese Indikation vor.

Hinsichtlich der Häufigkeit der Indikationen haben die psychischen Erkrankungen bereits den 2 . Platz bei der Invalidisierung erreicht, sodass eine wirksame Gegensteuerung notwendig ist.

\subsection{Bedarf an Case Managern zur Überwindung individueller Lücken}

Nicht so selten kommt es vor, dass Patienten nach der Entlassung aus dem Akutkrankenhaus wegen längeren Wartezeiten oder fehlender Information keinen Rehabilitationsaufenthalt angeboten bekommen und in der "Warteschlange" verweilen müssen. Um diesem Umstand wirksam abzuhelfen, wurde von Experten das Modell des Case Management entwickelt. Im Einzelfall soll dami die Patientenorientierung und -partizipation sowie die Ergebnisorientierung im österreichischen komplexen und arbeitsteiligen Sozialsystem verbessert werden. Die SV-Träger (zB Oberösterreichische Gebietskrankenkasse, Steiermärkische Gebietskrankenkasse, SVA, SVB) haben bereits rund 100 Case Manager beschäftigt.

\subsection{Rechts- und Zuständigkeitsprobleme}

Wie bereits unter Kapitel 1 und 3 aufgezeigt, hat die medizinische Rehabilitation in den drei Zweigen der SV unterschiedliche Strukturen und Zuständigkeiten. Während in der UV ein Rechtsanspruch besteht, ist dies trotz vielfacher Bemühungen der Behindertenverbände in der Kranken- und PV bis heute nicht erfolgt. Obwohl die Erläuterungen zur 50. ASVG-Novelle, die die medizinische Rehabilitation in der KV ab 1. Jänner 1992 eingeführt hat, von der Umwandlung zu einer Pflichtleistung ab 1. Jänner 1994 ausgegangen sind, hat der Gesetzgeber diese Absicht nicht weiterverfolgt. In der Kranken- und PV ist daher die medizinische Rehabilitation eine Ermessensleistung, die auch von den Finanzen der jeweiligen SV-Träger abhängig ist, was insb die Alterspensionisten bei einigen Gebietskrankenkassen zu spüren bekamen. Die PVA verwies wiederum darauf, dass kein genereller Gesetzesauftrag zur medizinischen Rehabilitation der Alterspensionisten vorliegt und verlangte eine Gesetzesänderung. Der HV der österreichischen SV-Träger arbeitete mit der PVA und dem Gesundheitsministerium einen Vorschlag zur Abtretung der medizinischen Rehabilitation der Alterspensionisten an die PV-Träger aus, der aber bis jetzt nicht realisiert werden konnte. Das folgende Tableau zeigt einen Vergleich de lege lata und de lege ferenda: 


\begin{tabular}{|c|c|}
\hline le Rechtslage & beabsichtigte Novellierung \\
\hline $\begin{array}{l}\text { KV: } \$ 154 \text { a ASVG } \\
\text { Versicherte (subsidiär), Pensionsbezie- } \\
\text { her, ausgenommen Invaliditätspensions- } \\
\text { bezieher, Angehörige }\end{array}$ & $\begin{array}{l}\text { KV: } \$ 154 \text { a ASVG } \\
\text { Versicherte (subsidiär), Hinterbliebe- } \\
\text { nenpensionsbezieher, Angehörige }\end{array}$ \\
\hline $\begin{array}{l}\text { PV: } \$ 300 \text { ASVG } \\
\text { (erwerbstätige) Versicherte und Bezieher } \\
\text { einer Invaliditätspension }\end{array}$ & $\begin{array}{l}\text { PV: } \$ 300 \text { ASVG } \\
\text { (erwerbstätige) Versicherte, Bezieher } \\
\text { einer Invaliditätspension, Bezieher einer } \\
\text { Eigenpension (Alterspension) }\end{array}$ \\
\hline $\begin{array}{l}\text { PV: } \$ 302 \text { Abs } 1 \text { Z } 2 \text { ASVG } \\
\text { Hilfsmittel werden an erwerbstätige } \\
\text { Versicherte und Bezieher einer Invalidi- } \\
\text { tätspension gewährt }\end{array}$ & $\begin{array}{l}\text { PV: } \$ 300 \text { Abs } 2 \text { ASVG } \\
\text { Hilfsmittel werden an erwerbstätige Ver- } \\
\text { sicherte und Bezieher einer befristeten } \\
\text { Invaliditätspension gewährt }\end{array}$ \\
\hline $\begin{array}{l}\text { KV: } \$ 154 a \text { Abs } 2 \\
\text { Hilfsmittel werden an Versicherte (sub- } \\
\text { sidiär) und Pensionsbezieher (außer } \\
\text { Invaliditätspensionsbezieher) gewährt }\end{array}$ & $\begin{array}{l}\text { KV: } \$ \text { 154a ASVG } \\
\text { Hilfsmittel werden an Versicherte } \\
\text { (subsidiär), an Bezieher einer dauernd } \\
\text { zuerkannten Invaliditätspension, } \\
\text { Eigenpension (Alterspension) oder Hin- } \\
\text { terbliebenenpension gewährt }\end{array}$ \\
\hline
\end{tabular}

\subsection{Notwendigkeit einer stärkeren Kooperation bei gleicher Qualität}

Auf dem Gebiet der stationären Rehabilitation gibt es nur einige Einrichtungen von privaten Trägern und kaum privatwirtschaftliche Beteiligungen. Wenngleich der privatwirtschaftliche Einfluss auf eigene Einrichtungen der SV nicht übersehen wird - zB gemeinsame Einkaufe zwecks Preisvorteilen - könnte auch sonst ein größerer Erfahrungsaustausch mit Privaten stattfinden, der nich nur allein über die Fachgesellschaften ablaufen sollte. Besonders wo die medizinische Rehabilitation indikationsmäßig noch nicht flächendeckend ausgebaut ist ( $\mathrm{zB}$ psychiatrische Rehabilitation) bietet sich eine verstärkte Zusammenarbeit mit privaten Rechtsträgern an. Allerdings muss die Kooperation bei gleicher Qualität stattfinden, weil sie sonst nicht zielführend wäre.

\subsection{Aufbau eines gezielten Netzwerks für alle Rehabilitationsfälle}

Der Rehabiliations-Case-Manager sollte keine Einzelerscheinung bleiben. Er müsste in die SV-Gesetze implementiert werden und es müsste flächendeckend die Betreuung für alle "Rehabilitationswerber" sichergestellt sein Es sollte daher eine Vernetzung der Akutkrankenhäuser mit den SV-Trägern und ihren Case Managern aufgebaut werden, um für jeden Spitalsabgänger die "maßgeschneiderte“ Rehabilitation ohne zeitliche Verzögerung gewährleisten zu können. Es wäre auch denkbar, dass der Case Manager die Voraussetzungen für eine ambulante Rehabilitation prüft, um dem Grundsatz „soviel ambulant wie möglich und sowenig stationär wie nötig“, der auch ökonomisch von Bedeutung wäre, zu entsprechen. Auch die bessere Vernetzung der KV mit der PV wäre ein Gebot der Stunde, um die Früherfassung für die medizinische Rehabilitation effizienter durchführen zu können.

\section{Literatur}

Bachner, Allgemein vertr(a)ägliche Grundlagen von PPP-Modellen, ecolex, 2005 ( $\mathrm{H} 4)$

M. Binder, Krankenversicherung, in Tomandl (Hrsg), System des österreichischen Sozialversicherungsrechts (21. ErgLfg 2008)

Fried, Chancen und Risken von PPP-Modellen im Gesundheitswesen, ÖKZ 2004 (H 9)

Horner, Ausgliederung und Ingerenz, Wolf Theiss Award - Band 6 (2004)

Lienbacher, Legalität im Sozialversicherungsrecht, in Kneihs/Lienbacher/Runggal$\operatorname{dier}$ (Hrsg), Wirtschaftssteuerung durch Sozialversicherungsrecht? (2005)

Maksimovic/Felix, Neuregelung der Krankenanstaltenfinanzierung ab 2005 im Rahmen der Gesundheitsreform, SozSi 2005 (H 3)

Müller, Möglichkeiten und Erfahrungen mit der ambulanten Rehabilitation in Österreich, SozSi 1999 (H 11)

Neurorehabilitation in Österreich, ÖBIG, im Auftrag des Strukturfonds (2004)

Oberndorfer, Das Verfahren im Sozialversicherungsrecht, in Tomandl (Hrsg) System des österreichischen Sozialversicherungsrechts (21. ErgLfg 2008)

Popper, Medizinische Rehabilitation in der Krankenversicherung - ein „Fall“ für Case Management, SozSi 2005 (H 10)

Rehabilitationsplan 2004 und 2009, ÖBIG, im Auftrag des HV

Rudda, Pensions- und Pflegevorsorge (2008)

Rudda, Rahmenbedingungen der medizinischen Rehabilitation in Österreich, SozSi 2005 (H 10)

Statistisches Handbuch der österreichischen Sozialversicherung (2008)

Tscharnutter et al, „Die Zeit danach“, PRIKRAF-Jahrbuch 2003

Walter/Mayer/Kucsko-Stadlmayer, Grundriss des österreichischen Bundesverfassungsrechts ${ }^{10}(2007)$

Teschner/Widlar/Pöltner, ASVG (105. ErgLfg 2008)

Teschner/Widlar, GSVG (90. ErgLfg 2008)

Teschner/Widlar, BSVG (70. ErgLfg 2009) 


\section{Medizinische Rehabilitation - die Rechtslage im deutschen SV-Recht}

\section{Einleitung}

Medizinische Rehabilitation ${ }^{1}$ ) ist ein Rechtsbegriff, der im Sozialverwaltungsrecht wiederholt, in verschiedenen Gesetzen, insb auch in den Regelungen der SV, verwendet wird. Dieser Begriff bezeichnet - für sich betrachtet, also ohne Berücksichtigung des normativen Kontextes, in dem er seine spezifische Bedeutung entfaltet - einen Vorgang, bei dem vor allem mit medizinischen Mitteln auf den Zustand einer Person eingewirkt wird, um bestimmte Defizite zu beseitigen, zu verringern oder ihrer Entstehung vorzubeugen.

Die derzeitige Rechtslage, soweit sie die medizinische Rehabilitation betrifft, ist kompliziert. Sie ist das Ergebnis einer jahrzehntelangen Entwicklung, die aus verschiedenen gesundheitspolitischen Strömungen gespeist worden ist, im Ergebnis aber zu erheblichen - auch praktischen - Problemen im Verwaltungsvollzug geführt hat.

\section{Die traditionelle Vorstellung vom Standort}

\section{der Rehabilitation in der SV}

Die Analyse der sv-rechtlichen Bestimmungen insoweit, als dort die medizinische Reha geregelt ist, führt zu einem Ergebnis, das - gleichsam in einem ersten gedanklichen Zugriff - folgendermaßen beschrieben werden kann: Die einschlägigen Rechtsnormen stimmen insoweit überein, als mit „medizinischer Rehabilitation" ein Kernbereich von Interventionen erfasst wird, der durch medizinische intendierte Maßnahmen geprägt ist; gemeinsam ist diesem begrifflichen Verständnis weiterhin, dass es um einen bestimmten defizitären Körperzustand geht, an dem diese Maßnahmen ansetzen und der - selbstverständlich in positiver Richtung - beeinflusst werden soll.

1) Die als allgemein verstandene begriffliche Eingrenzung auf "Leistungen zur Teilhabe behinderter oder von Behinderung bedrohter Menschen“ ist „SGB IX - Positivismus“; vgl zB Reimann in v. Maydell ua (Hrsg), Sozialrechtshandbuch (SRH) ${ }^{4}(2008) \$ 28$ $\mathrm{Rn}$ 1; zum Zusammenhang von „Behinderung, Rehabilitation und soziale Sicherung Behinderter"; vgl auch Schulin, Soziale Sicherung der Behinderten, Gutachten, erstattet im Auftrag des Deutschen Sozialgerichtsverbandes (1980) $16 \mathrm{ff}$. 


\subsection{Trennung von "Akutversorgung” und Rehabilitation}

Nach dem überkommenen, gleichsam historisch gewachsenen Verständnis schließt sich die Rehabilitation an die so genannte Akutversorgung an. Dabei versteht man unter „Akutversorgung“ Behandlungsmaßnahmen, die schwerpunktmäßig von Ärzten (oder deren Heilhilfspersonen) erbracht werden, ambulant oder stationär. Demgegenüber werde Maßnahmen im Rahmen der Rehabilitation zwar auch in der Verantwortung von Ärzten, praktisch jedoch schwerpunktmäßig von anderen Berufsgruppen erbracht, wobei traditionell eher die stationäre Behandlung im Vordergrund steht.

Diese Trennung scheint auch eine - ebenfalls historisch gewachsene - organisatorische (man könnte auch sagen: institutionelle) Parallele zu haben; die einschlägigen Begriffe könnten gleichsam „institutionell geprägt“ sein:

Die Akutversorgung" war nach dem früher ganz herrschenden Verständnis Aufgabe der gesetzlichen KV; die Rehabilitation war ausdrücklich Aufgabe der gesetzlichen Rentenversicherung, dort allerdings traditionell mit der spezifischen Zielsetzung, die rentenversicherungsrechtlich relevante Erwerbsfähigkeit wiederherzustellen, um Geldleistungen der gesetzlichen Rentenversicherung überflüssig zu machen ${ }^{2}$.

Gleichwohl gab es auch im früheren Recht der gesetzlichen KV Leistungen zur Rehabilitation ${ }^{3}$ ); weiterhin muss man wohl zugestehen, dass eine Unterscheidung zwischen Kranken- oder Heilbehandlung einerseits und medizinischer Redung zwischen Kranken- oder Heilbehandlung einerseits und medizition andererseits praktisch schwierig, wenn nicht gar unmöglich ist ${ }^{4}$ ).

In der Sache nach handelt es sich auch bei Maßnahmen der medizinischen Rehabilitation (im „klassischen“ Sinn des Rentenversicherungsrechts) um Maß nahmen, bei denen es um die Behandlung einer Krankheit geht und die deshalb durchaus auch als „Heilbehandlung“ im Sinne einer ganzheitlichen medizinischen Versorgung zu verstehen sind. Weiterhin ist heutzutage wohl allgemein insb aus medizinisch-fachlicher Sicht, anerkannt, dass die eine Behandlungsform (Akutbehandlung) und die andere Behandlungsform (Rehabilitation) jeweils auch Elemente der jeweils anderen Behandlungsformen umfassen, so dass im Einzelfall eine klare Abgrenzung zwischen der Akutbehandlung und der medizinischen Rehabilitation aus medizinischer Sicht zweifelhaft und aus juristischer Sicht gleichsam ,rechtstechnisch“ schwierig ist ${ }^{5}$ ).

Gleichwohl liegt auch dem geltenden Recht der medizinischen Rehabilitation diese Unterscheidung zugrunde; sie scheint somit nach Auffassung des Gesetzgebers möglich und notwendig zu sein; jedenfalls geht die Gesetzeslage offensichtlich nach wie vor von dieser Differenzierung aus.

2) Das wird mit der Zielsetzung "Rehabilitation vor Rente" (vgl nunmehr $\$ 9$ Abs 1 S SGB VI) gesetzlich festgelegt; sa $\$ 26$ Abs 3 SGB VII sowie $\$ 8$ Abs 2 SGB IX

$\left.{ }^{3}\right)$ Dabei ist vor allem an diejenigen Kuren zu denken, die der Sache nach rehabilitativen Zielsetzung verfolgen.

$\left.{ }^{4}\right)$ Vgl für alle Schulin, Handbuch des SV-Rechts I (KV-Recht) $\$ 6 \mathrm{Rn} 168 \mathrm{mwN}$

5) Reimann, aaO $\$ 28 \mathrm{Rn} 118$.

\subsection{Fragwürdigkeit der Trennung}

Zwei Beispiele mögen die Problematik dieser Differenzierung sowohl aus medizinischer als auch aus rechtlicher Betrachtungsweise belegen: Zum einen regelt - und somit der Tradition folgend - das Recht der gesetzlichen Rentenversicherung, dass dieser Bereich keine Leistungen zur medizinischen Rehabilitation in der Phase der akuten Behandlungsbedürftigkeit und somit unter Umständen anstelle einer ansonsten erforderlichen Krankenhausbehandlung erbringen darf $\left.^{6}\right)$. Und auch die stationäre Heilbehandlung, die unmittelbar im Anschluss an eine Krankenhausbehandlung medizinisch notwendig ist und von dem Träger der Rentenversicherung gewährt werden soll, wird - und insoweit aus der traditionellen Sicht des Rentenversicherungsrechts folgerichtig - als „Anschlussrehabilitation " bezeichnet und geregelt ${ }^{7}$ ).

Im Bereich der gesetzlichen KV wird jedoch das, was an Leistungen der medizinischen Rehabilitation zu erbringen ist, zwar als integraler Bestandteil der medizinischen Versorgung gesehen, die von Beginn an "ganzheitlich" gestaltet werden muss; gleichwohl werden auch hier verschiedene Phasen der Rehabilitation ausdrücklich begrifflich-tatbestandsmäßig definiert und hinsichtlich der Rechtsfolgen (Pflichten/Ansprüche) geregelt und zwar als so genannte „Frührehabilitation“ sowie als so genannte Anschlussheilbehandlung (oder auch „Anschlussrehabilitation") ${ }^{8}$ ).

Im Übrigen wird heutzutage gelegentlich auch noch eine dritte Phase erkannt, die zwischen der Phase akuter Behandlungsbedürftigkeit und der Phase der medizinischen Rehabilitation legt. Diese „Zwischenphase“ soll sich durch eine besonders enge Verzahnung der kurativ-medizinischen und der Rehabilitierungsmaßnahmen auszeichnen. Beispiel hierfür sind die Phasenmodelle der neurologischen und kardiologischen Rehabilitation ${ }^{9}$ ).

\subsection{Fehlen eines Rehabilitations- „Grundtatbestandes“}

Bemerkenswert ist auch, dass es für diesen Gesundheitszustand, der Anlass für Maßnahmen und Leistungen der medizinischen Rehabilitation ist und der dadurch gekennzeichnet ist, dass - traditionell - nach einer Akutversorgung (nach heutiger Erkenntnis und Rechtslage auch bereits zeitgleich mit einer Akutversorgung) noch spezifische gesundheitlich-medizinische Defizite bestehen, kein eigenständiger Begriff gebildet worden ist, der dem Begriff der "Krankheit" (dem Leitbegriff der gesetzlichen KV) entspricht und somit vergleichbar ist.

Man kann dieses Fehlen einer eigenständigen Bezeichnung für diese körperliche, geistige oder seelische Situation eines Menschen für irrelevant halten mit der Überlegung, dass es sich dabei zwar auch noch um „Krankheit“ handelt, deren Behandlung jedoch nicht mehr, jedenfalls nicht schwerpunktmäßig, in den Aufgabenbereich der gesetzlichen KV fällt. Es ist aber ersichtlich, dass man damit

$\left.{ }^{6}\right) \$ 13 \mathrm{Abs} 2 \mathrm{Nr} 1$ und $2 \mathrm{SGB}$ VI.

7) Vgl $\$ 32$ Abs 1 Satz 2 1. HS SGB VI (Zuzahlung bei Leistungen zur medizinischen Rehabilitation und bei sonstigen Leistungen).

$\left.{ }^{8}\right) \S 40$ Abs 6 SGB V sowie auch $\$ 32$ Abs 1 S 2 SGB VI.

9) Reimann, $\$ 28 \mathrm{Rn} 119 \mathrm{mwN}$ in Fn 44. 
darauf verzichtet, eine spezifische Tatbestandsmäßigkeit für diese Situation (und somit für spezifische gesundheitliche Defizite) in der Rentenversicherung zu definieren, an die die ebenfalls spezifischen Leistungen der gesetzlichen Rentenversicherung gesetzestechnisch und in der praktischen Umsetzung auch tatsächlich anknüpfen.

\subsection{Die Situation in der gesetzlichen Unfallversicherung}

$\mathrm{Zu}$ erwähnen ist in diesem Zusammenhang, dass im Bereich der gesetzlichen UV traditionell eine Trennung von „Akutversorgung “ und anschließender Maßnahmen zur endgültige Wiederherstellung der körperlichen, geistigen und seelischen Gesundheit nicht vorgenommen wird. In diesem Rechtsgebiet (gesetzliche UV) geht es ähnlich wie im Bereich der Rentenversicherung dort, wo diese Leistungen der medizinischen Rehabilitation erbringt, auch darum, die Erwerbsfähigkeit eines Menschen, die er durch einen Arbeitsunfall oder eine Berufskrankheit eingeschränkt ist, wiederherzustellen - dies im Übrigen auch zur Vermeidung einer Rente wegen „Minderung der Erwerbsfähigkeit" ${ }^{\text {"10 }}$ ).

Letztlich ist allerdings - und insoweit ist die Rechtslage in der UV anders als in der Rentenversicherung - dieses Ziel (Vermeidung von Rentenleistung) nicht allein maßgeblich; es kommt bei den Rehabilitationsleistungen nach dem SGB VII allein darauf an, die Verletzungsfolgen zu bessern oder die Beschwerden zu lindern ${ }^{11}$ ) Eine auch definitorisch-gesetzestechnische Trennung von Maßnahmen der Heilbehandlung in verschiedene Phasen ist verständlicherweise als überflüssig betrachtet worden angesichts der organisatorisch-institutionellen alleinigen Zuständigkeit der Träger der gesetzlichen UV für die gesamte Heilbehandlung. Vielmehr hat man das gesamte Heilverfahren (sowie wohl auch dessen Ergebnis) als „Rehabilitation" bezeichnet ${ }^{12}$ ). Und der im Recht der gesetzlichen UV ebenfalls geltende Grundsatz der „Rehabilitation vor Rente ${ }^{\text {“13 }}$ ) bezieht sich somit auf sämtliche Maßnahmen der Heilbehandlung, also auch unter Einschluss der Leistungen, die man durchaus auch als solche der "Akutversorgung" bezeichnen kann.

Erst in jüngerer Zeit ${ }^{14}$ ) ist innerhalb der „Heilbehandlung ${ }^{(15}$ ) diese Trennung zwischen „Akutbehandlung“ und nachfolgender Rehabilitation eingeführt worden.

$\left.{ }^{10}\right) \mathrm{Vgl} \$ 2$ Abs 2 („Rehabilitation vor Rente“), $\$ 56-62$ (Renten an Versicherte) SGB VII.

11) Ruland/Schulz-Weidner in Schulin, Handbuch des SV-Rechts II (UV-Recht) (1996) § $10 \mathrm{Rn} 16$

12) Vgl auch die Überschrift zu $\$ 1$ SGB VII: „Prävention, Rehabilitation, Entschädigung“.

13) $\$ 26 \mathrm{Abs} 3 \mathrm{SGB}$ VII

$\left.{ }^{14}\right)$ Mit der Einführung des SGB IX, vgl 3.1; eine eindeutige Abgrenzung zwischen Heilbehandlung und Rehabilitation(smaßnahmen) sowie eine einheitliche Terminologie sind nicht möglich, ebenso Benz in Hauck/Noftz, Sozialgesetzbuch - Siebtes Buch. Gesetzliche UV. (SGB VII) Kommentar K\$27 Rn 4-4c; dieser Ansicht ist übrigens auch der Gesetzgeber selbst, vgl Reg - Entwurf zum SGB VII, BT-DS 13/2204, 83.

15) Nach den Regelungen des SGB VII, vgl 26, 27 - 34 SGBVII.

\section{5 "Behinderung" als Rehabilitations- „Grundtatbestand“?}

Nach der neueren, derzeitigen Systematik des gesamten Rechtskomplexes der medizinischen Rehabilitation scheint es so zu sein, dass dieser Zustand körperlicher, geistiger und seelischer Defizite mittlerweile eine allgemeine Definition erhalten hat. Der für den gesamten Bereich der Rehabilitation prägende $\mathrm{Be}$ griff ist der der „Behinderung $\left.{ }^{\text {"16 }}\right)$. Wenn diese Einschätzung zutreffend ist, dann sind die Maßnahmen der Rehabilitation solche, die sich auf eine Behinderung - nach der geltenden Rechtslage im Übrigen auch auf eine lediglich „drohende“ Behinderung ${ }^{17}$ ) - beziehen und die sich an die medizinische Akutversorgung von Personen anschließen, bei denen die an sich denkbare und als möglich erscheinende Herstellung, insb die Wiederherstellung eines früheren „besseren“ gesundheitlichen Zustandes, allein mit Maßnahmen aus dem mit „Akutversorgung" bezeichneten Aktions- oder Interventionsbündel nicht bewerkstelligt werden konnte.

\subsection{Fortgang der Erörterung}

Nach diesen Überlegungen zu einem gleichsam gemeinsamen Nenner des gesamten Rechts der medizinischen Rehabilitation sollen die einzelnen Rechtsbereiche betrachtet werden, in denen dieser Leistungskomplex Gegenstand gesetzlicher Regelungen ist. Dabei werden jeweils sowohl die materiell-rechtlichen Bestimmungen als auch die organisationsrechtlichen (die Aufbau- und Ablauforganisation betreffenden) Regelungen dargestellt.

Um die rechtliche und rechtspraktische Problematik dieses gesamten Komplexes erfassen zu können, insb im Hinblick auf Mehrfach-Zuständigkeiten - im heutigen "modernen“ Sprachgebrauch: „Schnittstellen" - und hinsichtlich der im Gesetz vorgesehenen verfahrensrechtlichen Lösungen, ist eigentlich eine Darstellung sämtlicher einschlägiger Teilbereiche erforderlich, in denen Leistungen der medizinischen Rehabilitation rechtlich vorgesehen und tatsächlich auch gewährleistet werden.

Gleichwohl wird im nachfolgenden die Betrachtung auf die Bereiche der SV also auf die gesetzlichen Kranken-, Renten- und UV sowie auch die Pflegeversicherung beschränkt, so dass die Bereiche der Sozialhilfe („Fürsorge $\left.\left.{ }^{c}\right)^{18}\right)$, der Jugendhilfe ${ }^{19}$ ) und der sozialen Entschädigung ${ }^{20}$ ) (dieser Bereich ist ein wiederum aus mehreren Gesetzen bestehendes Rechtsgebiet) nicht erörtert wird.

$\left.{ }^{16}\right)$ Die Ansicht, dass „Rehabilitation grundsätzlich an Behinderung anknüpft“, vertritt zB Noftz in ders/Hauck, Sozialgesetzbuch - Fünftes Buch. GKV (SGB V) K\$ 40 Rn 10 der zugleich aber auch „vielfache Überschneidungen“ feststellt.

$\left.{ }^{17}\right) \mathrm{Vgl} \$ 2$ Abs 1 SGB VII.

$\left.{ }^{18}\right) \mathrm{Vgl} \$ \$ 8,13,14,15,19$ Abs 3 SGBXII.

$\left.{ }^{19}\right) \mathrm{Vgl} \$ 35 \mathrm{a}$ SGB VIII.

$\left.{ }^{20}\right) \mathrm{Vgl} § 27 \mathrm{~d}$ Abs $1 \mathrm{Nr} 3$, Abs $3 \mathrm{~S}$. 1 BVG iVm SGB XII, 3. Abschnitt. 


\section{Der rechtliche „gemeinsame Nenner} der medizinischen Rehabilitation

Bei der Suche nach einem rehabilitationsrechtlichen "Grund-Tatbestand“ innerhalb des gesamten Regelungskomplexes, der sich mit medizinischer Rehabilitation befasst, sowie im Hinblick auf Überlegungen zu einem einheitlichen Leistungssystem in diesem Bereich fällt das SGB IX ins Auge. Von diesem gedanklichen Ausgangspunkt her soll den oben erläuterten Fragestellungen nachgegangen werden.

\subsection{Die Rechtsgrundlage im SGB IX}

Nach der derzeitigen Rechtslage ist die medizinische Rehabilitation regelungstechnisch ,vor die Klammer gezogen“ und somit einheitlich für das gesamte Sozialverwaltungsrecht ${ }^{21}$ ) festgelegt, und zwar im "Sozialgesetzbuch IX - Rehabilitation und Teilhabe behinderter Menschen “"22); dieses Gesetz ist im Wesentlichen am 1. Juli 2001 in Kraft getreten.

Zugleich wurde damit das am 1 . Oktober 1974 in Kraft getretene „Rehabilitations-Angleichungsgesetz (RehaAnglG)“ abgelöst; dieses RehaAnglG war ein erster, relativ weitgehender Versuch einer Angleichung des Leistungsrechts ohne jedoch das gegliederte System der Leistungsträger und die daraus sich er gebende Zuständigkeitsordnung zu ändern. Einzelheiten der Entwicklung der Rehabilitation bis zum RehaAnglG sowie in der nachfolgenden Zeit bis hin zum SGB XI und damit zur derzeitigen Rechtslage sollen hier nicht nachgezeichnet werden ${ }^{23}$ ).

Erwähnt werden soll lediglich, dass mit dem RehaAnglG die Krankenkassen auch offiziell als Rehabilitationsträger anerkannt worden sind, so dass seitdem die Leistungen der Krankenkassen und der - traditionell für die medizinische Reha zuständigen - Rentenversicherungsträger sowohl für erwerbstätige als auch nicht (mehr) erwerbstätige Versicherte grundsätzlich gleichwertig sein sollten ${ }^{24}$ ).

\subsection{Materielles Recht -}

\section{ein Leistungsfall "der" medizinischen Rehabilitation?}

Das SGB IX enthält in seinem 4. Kapitel die Bestimmungen zu den „Leistungen zur medizinischen Rehabilitation" - so die Überschrift zu diesem Kapitel. In den $\$ \$ 26-31$ SGB IX werden aber nicht nur die Leistungen im Einzelnen aufgeführt ${ }^{25}$ ), also die Behandlung von Ärzten, Zahnärzten, Angehörigen anderer

${ }^{21}$ ) Soweit es im SGB erfasst ist, vgl auch $\$ 68$ SGB I.

${ }^{22}$ ) V 19.6. 2001, BGBl I 1046.

23) Vgl dazu im Einzelnen Lachwitz/Welti (Hrsg), Handkommentar zum SGB IX (HK-SGB XI) (2002) 3-12.

${ }^{24)}$ Lachwitz/Welti, aaO 5.

$\left.{ }^{25}\right) \$ 32$ SGB IX enthält eine Verordnungsermächtigung zur Klärung von Abgrenzungsproblemen zwischen den Leistungsträgern, die zur Früherkennung und Frühförderung von (auch nur drohenden) Behinderungen gem $\$ 30$ Abs 1 und 2 SGB IX zuständig sind.
Heilberufe ${ }^{26}$ ), Früherkennung und Frühförderung, Arznei- und Verbandsmittel, Heilmittel einschließlich physikalischer Therapie, Sprach- und auch Beschäftigungstherapie, Psychotherapie, Hilfsmittel, Belastungserprobung und Arbeitstherapien ${ }^{27}$ ), sondern es werden mit $\$ 26$ Abs 1 SGB IX zugleich weitere Fragen beantwortet.

Zum einen werden die Inhaber des (komplexen) Anspruchs auf medizinische Rehabilitation, also die Gläubiger dieser Leistungen, bezeichnet - es sind die behinderten sowie die von Behinderung bedrohten Menschen.

Zum anderen werden eine Reihe weiterer rechtsverbindlicher Voraussetzungen genannt, dies geschieht in Form von Zielsetzungen, also zum Beispiel „Behinderungen abwenden“" oder „Einschränkungen von Erwerbsfähigkeit oder den vorzeitigen Bezug von Sozialleistungen vermeiden"; diese Zielsetzungen sind als Alternativen normiert - eines dieser Ziele muss jedoch erreichbar, also auch von Rechts wegen prognostizierbar sein, um einen Anspruch entstehen zu lassen.

\subsection{Wirkung des SGB IX in den Besonderen Teilen des SGB}

Wichtig ist es darauf hinzuweisen, dass es sich hierbei (weniger bei der Behinderung" im Sinne des $\$ 2$ Abs 1 SGB IX, sondern vielmehr bei $\$ 26$ SGB IX) um einen abstrakt normierten Leistungsfall handelt, dessen begriffliche Regelungsbestandteile nur insoweit Verbindlichkeit in den Besonderen Teilen des SGB haben, als dort diese Begriffe verwendet werden; darauf beschränkt sich die Wirkung dieser Norm.

Diese Vorschrift ist zwar wie eine Anspruchsgrundlage formuliert; sie entfaltet jedoch - für sich betrachtet - diese Wirkungen nicht gleichsam ,aus sich heraus", sondern für einen wirklichen, letztlich rechtlich gleichsam „real-existierenden" Anspruch auf Leistungen der medizinischen Rehabilitation ist es erforderlich, dass eine Vorschrift in einer Regelung eines Besonderen Teils des SGB einen Anspruch auf diese Leistungen einräumt - damit wird der hier dargelegte Leistungskomplex des SGB IX rechts- und gesetzestechnisch in das jeweilige besondere Sozialverwaltungsrecht eingefügt.

Man kann es auch so ausdrücken, dass der SGB IX-Leistungsfall latent (oder virtuell) normiert ist, dass er jedoch tatbestandsmäßig real wird durch die Bezugnahme einer Regelung in einem der Besonderen Teile auf das SGB IX.

Beispiele hierfür sind $\$ \$ 27$ Abs 1,33 SGB VII, $\$ 15$ SGB VI, $\$ 40$ SGB V.

In diesem Zusammenhang ist allerdings auch darauf hinzuweisen, dass die Besonderen Teile Regelungen treffen können, in denen von den Vorschriften des SGB IX abgewichen wird; in diesen Fällen werden die an sich als einheitlich konzipierten und formulierten Anspruchsinhalte insoweit verdrängt - diesen SGB IX. 30, 31 SGB IX. 
Vorbehalt und vor allem diesen Vorrang der Besonderen Teile legt $\$ 7$ SGB IX ausdrücklich fest ${ }^{28}$ ).

Und noch etwas Weiteres ist auch bereits in diesem Zusammenhang zu bedenken: $\mathrm{Ob}$ ein Anspruch real gegeben ist, ob also ein Leistungsfall wirklich besteht, kann zusätzlich von weiteren bereichsspezifischen Voraussetzungen abhängig sein; beispielsweise ist für einen Anspruch gem $\$ 27 \mathrm{Abs} 1 \mathrm{Nr} 7, \$ \$ 28 \mathrm{ff}$. SGB VII ${ }^{29}$ ) unter anderem auch Voraussetzung, dass die zur Rehabilitationsleistung führende (unter Umständen nur drohende) Behinderung im Rechtssinn zurückzuführen ist entweder auf einen Arbeitsunfall oder auf eine Berufskrankheit ${ }^{30}$ ).

\subsection{Einzelfragen zu $\$ 26$ SGB IX}

3.4.1 Konkretisierung unbestimmter Gesetzesbegriffe durch Verwaltung und Gerichte. Das Anliegen des SGB IX

Die Rechtsbegriffe des $\$ 26$ SGB IX, die in dessen Abs 1 der Gesetzgeber die Zielsetzungen und in Abs 2 und 3 die Leistungen der medizinischen Rehabilitation festgelegt hat, sind mehr oder weniger unbestimmt. Hinsichtlich der Konkretisierung dieser Begriffe durch Auslegung seitens der Verwaltung (durch einzelne Entscheidungen, Satzungen, Verwaltungsvereinbarungen $\left.{ }^{31}\right)$ ) sowie der Gerichte ist zu bedenken, dass (ganz praxisbezogen betrachtet) derartige Bemühungen, also solche gedanklichen Vorgänge, jeweils ihren tatsächlichen und rechtlichgedanklichen Ausgangspunkt in einem besonderen Rechtsgebiet (Kranken-, Renten- oder UV-Recht) haben; und es ist nicht ausgeschlossen, dass dabei übernommene, in der Vergangenheit "gewachsene" Vorstellungen vom zutreffenden Verständnis rehabilitationsrelevanter Begriffe maßgebend sind.

Grundsätzlich sollte allerdings eine einheitliche Begrifflichkeit angestrebt werden, entsprechend dem ,strategischen" Anliegen des SGB IX, das Rehabilitationsrecht möglichst weitgehend zu vereinheitlichen.

\subsection{2 "Offene“ Leistungskataloge}

Weiterhin ist bemerkenswert, dass die gesetzlich vorgesehenen Maßnahmen in den Leistungsbündeln, die in $\$ 26$ SGB IX in Abs 2 und Abs 3 jeweils zusammengefasst sind, nicht abschließend normiert sind. Mit der Verwendung des Wortes „insb“ hat der Gesetzgeber nur die wichtigsten Leistungsfelder ${ }^{32}$ ) und

${ }^{28)}$ Vgl hierzu Welti/Felix, aaO $\$ 7$ Rn 8, 9.

$\left.{ }^{29}\right)$ Leistungen zur medizinischen Rehabilitation nach dem Recht der gesetzlichen UV; die "wirkliche“, bereichsspezifische Anspruchsgrundlage ist $\$ 26 \mathrm{Abs} 1$ Satz 1 in Verbindung mit $\$ 27 \mathrm{Abs} 1 \mathrm{Nr} 7$ SGB VII in Verbindung mit $\$ 26 \mathrm{Abs} 2 \mathrm{Nr} 1$ und $3-7$ und Abs 3 SGB IX

$\left.{ }^{30}\right)$ Auch dies iSd $\$ \$ 8,9$ SGB VII.

$\left.{ }^{31}\right)$ ZB gem $\$ 10$ SGB IX - Koordinierung der Leistungen, $\$ 13$ SGB IX - Gemeinsame Empfehlungen; vgl dazu Näheres bei 9.2.3.1.

32) ZB (auch) Früherkennung und Frühbehandlung, Abs $2 \mathrm{Nr}$ 2, Heilmittel, Abs 2 $\mathrm{Nr}$ 3, Belastungserprobung und Arbeitstherapie, Abs $2 \mathrm{Nr} 7$.
Leistungserbringer ${ }^{33}$ ) aufgeführt; das gilt auch für die so genannten Annexleistungen gem $\$ 26$ Abs 3 SGB IX ${ }^{34}$ ). Damit ist der Umfang der rechtlich zulässigen und gegebenenfalls gebotenen Maßnahmen der medizinischen Rehabilitation offen, und zwar in gleicher Weise auch für die Besonderen Teile des SGB normiert - es sei denn, dass dort (mit Vorrang gegenüber dem SGB IX) abschließende Regelungen getroffen worden sind, in denen Art und Umfang der Leistungen abweichend von $\$ 26$ SGB IX festgelegt wird.

\subsubsection{Auslegungsschwierigkeiten innerhalb des $\$ 26$ SGB IX}

Schließlich ist auch nicht ausgeschlossen, dass $\$ 26$ SGB IX selbst Unklarheiten enthält und juristische Rätsel aufgibt, die durch die (oben beschriebene) rechtstechnische Integration dieser Vorschrift in die leistungsrechtlichen Bestimmungen der Besonderen Teile nicht gelöst werden.

So besteht zum Beispiel die Unsicherheit hinsichtlich der Voraussetzungen für das Vorliegen einer „chronischen Krankheit“ und dabei wiederum beispielhaft die Frage nach den rechtlichen Anforderungen an eine "Sucht" sowohl aus dem Gesichtspunkt des SGB IX ${ }^{35}$ ) als auch aus der (auf den ersten Blick möglicherweise bereichsspezifischen) Perspektive zum Beispiel des SGB ${ }^{36}$ ).

\subsubsection{Fragen der Zuständigkeit (Trägerschaft)}

In diesem Zusammenhang ist - ergänzend zu den modellhaften, aus heutiger Sicht wohl zu einfachen, angesichts des geschriebenen Rechts unter bestimmten Umständen unzutreffende Vorstellungen vom Zusammenhang von Akutversorgung und Rehabilitation - zu bemerken, dass diese beiden tatsächlich und rechtlich analytisch trennbaren Vorgänge keineswegs in die Hand eines und desselben Leistungsträgers liegen müssen, so wie das beispielsweise nach wie vor bei der gesetzlichen UV der Fall ist.

Traditionell hat, wie bereits erwähnt, das deutsche SV-Recht eine diesbezügliche Aufgabenverteilung und -zuweisung dahingehend vorgenommen, dass die Akutversorgung der gesetzlichen KV und ihren Trägern und die - in der Regel wohl anschließende - Rehabilitation grundsätzlich und schwerpunktmäßig den Trägern der gesetzlichen Rentenversicherung obliegt; allerdings hatte die Rechtsprechung auch schon früher den Ausgleich einer Behinderung und des dadurch verursachten Funktionsausfalls als medizinische Leistung bewertet und der (seinerzeit so bezeichneten) Krankenpflege im Sinne der gesetzlichen KV zugeordnet $\left.{ }^{37}\right)$.

${ }^{33}$ ) Vor allem Ärzte und von denen beaufsichtigte (früher so genannte) „Heilhilfspersonen"; vgl Abs $2 \mathrm{Nr} 1$.

$\left.{ }^{34}\right)$ Stähler in Lachwitz/Schellhorn/Welti, aaO \$26 Rn 22

${ }^{35)} \mathrm{Vgl} \mathrm{Welti,} \mathrm{aaO} \$ 3 \mathrm{RdNr} 6,7$; Stähler, aaO $\$ 26 \mathrm{Rn} 11$.

${ }^{36)}$ Vgl KassKomm-Höfler, $\$ 40$ SGB V Rn 8.

$\left.{ }^{37}\right)$ BSG SozR $2200 \$ 182 \mathrm{Nr} 73 \mathrm{mwN}$; Näheres zur Entwicklung und normativen Ausgestaltung der Rehabilitation im KV-Recht siehe unten bei 5. sowie KassKomm-Höfler, $\$ 11$ SGB V Rn 12, 13 
Auf einem anderen Blatt steht allerdings die Frage, ob die Fragmentierung von Körperzuständen und die Zuordnung entsprechender Leistungskomplexe in einem gegliederten Versorgungssystem insoweit sinnvoll ist, als nicht ein einziger, sondern verschiedene Verwaltungsträger mit der Aufgabe betraut sind, diesbezügliche Leistungen zu gewähren; damit ist die Frage aufgeworfen, wie man das Leistungsgeschehen insgesamt sinnvoll, möglichst optimal, organisiert ${ }^{38}$ ).

Festhalten lässt sich jedenfalls, dass eine Kumulation von Leistungsfällen und diesbezüglichen Anspruchskomplexen gegeben sein kann, vom Gesetzgebe auch ausdrücklich oder der Sache nach bemerkt und geregelt worden ist - und dass die Vielfalt der Leistungsträger mit einer gewissen Zwangsläufigkeit zu organisatorischen Problemen führen kann, die ihren letzten Grund in einer (angesichts der derzeitigen Trägervielfalt) unvermeidbaren Mehrfach-Zuständigkeit von Leistungsträgern in einem Einzelfall haben mit Auswirkungen auf den Versicherten, dessen praktische „Versorgung“ deshalb mit systembedingten, spezifischen Schwierigkeiten belastet ist.

\section{Rehabilitation in der Gesetzlichen Rentenversicherung (GRV)}

\subsection{Der Standort der medizinischen Rehabilitation in der GRV}

Die Rentenversicherung zielt an sich - wie ihr Name schon sagt - offenbar in erster Linie auf die Erbringung von Geldleistungen (Renten) für Personen ab, die wegen des Ausscheidens aus dem Erwerbsleben wegen Alters oder verminderter Erwerbsfähigkeit ${ }^{39}$ ) und angesichts typischerweise nicht vorhandener sonstiger Einkommen einen staatlichen Lohnersatz erhalten sollen ${ }^{40}$ ).

Zur Vermeidung dieser Leistung (und zum Erhalt von Beitragszahlen) kennt das Rentenversicherungsrecht von Anfang $\mathrm{an}^{41}$ ) Vorschriften über die Durchführung von Heilverfahren ${ }^{42}$ ). Für die dabei getätigten Maßnahmen hat sich der Begriff der Rehabilitation durchgesetzt; von Beginn der SV mit ihren Teilbereichen Kranken-, Unfall- und Rentenversicherung an und bis heute wird damit abstrahierend und zusammenfassend ein Bündel von Maßnahmen bezeichnet - in der heutigen Terminologie spricht man von einer Komplexleistung.

Anders als im KV-Recht fehlt es jedoch (wie bereits erwähnt) traditionell, also nach wie vor, an einem leistungsbegründenden Grundtatbestand, gleichsam als Gegenstück und Ergänzung zur „Krankheit“. Allerdings war und ist es möglich aus einer Zusammenschau der Zielsetzungen und der Leistungen zur medizinischen Rehabilitation einen solchen Grundtatbestand zu ermitteln; dieser lässt sich - aus historischer Perspektive und auch heute noch - ungefähr als einen

${ }^{38}$ ) Vgl im Einzelnen unten 8. und 9.

39) Vgl $\$ 33$ SGB VI - Rentenarten sowie insgesamt den 2. Abschnitt des 2 . Kapitels SGB VI (\$ $\$ 33-105 a)$

${ }^{40}$ ) Bei den Hinterbliebenenrenten zeigt sich diese subsidiäre Funktion hinsichtlich der Anrechung von Einkommen. Vgl $\$ \$ 18 \mathrm{a}-18 \mathrm{c}$ SGB IV.

${ }^{41}$ ) Also seit dem „Reichsgesetz betreffend die Invaliditäts- und Alterssicherung“ vom 22.6. 1889, RGBl 97.

$\left.{ }^{42}\right)$ Pitschas in Schulin, Handbuch des SV-Rechts III, Rentenversicherungsrech (1999) § 31 Rn 4, spricht vom „Grundstock des Rehabilitationsrechts“.
Zustand beschreiben, der wegen seiner körperlichen, geistigen oder seelischen Defizite zu einer - unter Umständen nur teilweisen - Aufgabe oder erhebliche Einschränkung der Erwerbstätigkeit gezwungen hat; da auch die Gefahr eines Ausscheidens aus dem Berufsleben rechtlich dem Grunde nach gleichbehandelt wird, liegen dem Rehabilitationsrecht der gesetzlichen Rentenversicherung damit augenscheinlich zwei Grundtatbestände zugrunde, bei deren Vorliegen Leistungen zur Rehabilitation infrage kommen.

\subsection{Die Bedeutung der „Behinderung“ im SGB VI} und im Vergleich mit dem SGB IX

Nun drängt sich die Vermutung auf, dass diese beiden rentenversicherungsrechtlichen Grundtatbestände, bei deren Vorliegen Ansprüche auf Rehabilitationsleistungen entstehen, heutzutage identisch sind mit der „Behinderung" und der "drohenden Behinderung“ im Sinn des SGB IX. Die Prüfung dieser Hypothese ist an sich einfach; es sind lediglich die Leistungsvoraussetzungen für die medizinische Rehabilitation einerseits nach dem SGB IX und andererseits nach dem SGB VI miteinander zu vergleichen ${ }^{43}$ ), indem man - bildlich gesprochen - die Folie des SGB IX gleichsam auf die Regelungen des SGB VI legt.

Dieses Verfahren führt zu interessanten Ergebnissen; es zeigt sich nämlich, dass die leistungsrechtlichen Grundtatbestände der medizinischen Rehabilitation nach dem SGB VI nicht deckungsgleich sind mit den Grundtatbeständen nach dem SGB IX, die von dem Begriff der Behinderung ${ }^{44}$ ) ausgehen. Diese Prüfung führt vielmehr zu dem Ergebnis, dass es zwar - einerseits - Merkmale gibt die sowohl für das SGB IX als auch das SGB VI prägend sind, dass aber - andererseits - in beiden Rechtsgebieten auch Merkmale maßgebend sind, die nur in einem dieser Bereiche gegeben sind. Beide Regelungsgebiete haben somit eine gemeinsame "tatbestandsmäßige Schnittmenge“, enthalten aber auch bereichsspezifische Besonderheiten.

\subsection{Die Bedeutung der "Behinderung” in den leistungsrechtlichen "Grundtatbeständen" des SGB V}

Ein Blick auf die einschlägigen Regelungen zeigt Art und Umfang des rechtstechnischen Zusammenspiels zwischen SGB IX und SGB VI.

Gemäß $\$ 9$ Abs 1 Nr 1 SGB VI ist leistungsrechtlicher Grundtatbestand nicht nur die Auswirkung einer (körperlichen, geistigen oder seelischen) „Behinderung", sondern daneben, zusätzlich - also gleichsam als ein zweiter Grundtatbestand - die Auswirkungen einer Krankheit, also des kv-rechtlichen "GrundFalles".

Somit lässt sich also bereits in diesem Zusammenhang festzustellen, dass nach dem soeben Gesagten die „Behinderung“ des SGB IX offenbar nur eine

${ }^{43}$ ) Letztlich an sich auch unter Berücksichtigung sowohl der tatbestandlichen Voraussetzungen als auch der Regelungen zum Leistungsumfang, die ebenfalls Bedeutung für den im Einzelfall bestehenden Anspruch haben.

${ }^{44}$ ) Im Folgenden soll die „drohende Behinderung“ nicht ständig erwähnt werden. 
Teilmenge innerhalb der rentenversicherungsrechtlichen Grundtatbestände ist und jedenfalls nicht mit dem rentenversicherungsrechtlichen Grundtatbestand identisch ist.

Das ist auch insoweit bemerkenswert, als das SGB IX eigentlich auch als Allgemeiner Teil“ des Rehabilitationsrechts ${ }^{45}$ ) konzipiert worden ist. Wäre dieses gesetzgeberische Konzept realisiert worden, dann müsste die Behinderung (sowie die drohende Behinderung) einzig und allein integraler Bestandteil der rehabilitationsrechtlichen Regelungen der Besonderen Teile des SGB sein, soweit es die medizinische Rehabilitation angeht. Das würde im Übrigen durchaus nich ausschließen, dass, anknüpfend an die einheitlichen Grundtatbestände „Behinderung" und "drohende Behinderung", gleichwohl im SGB VI spezifische Sachverhalte normiert blieben dergestalt, dass Auswirkungen nur insoweit rentenversicherungsrechtlich relevant sind, als sich die körperlichen, geistigen oder seelischen Defizite auf die Erwerbsfähigkeit des Versicherten (negativ) auswirken.

\subsection{Tatbestandliche Voraussetzungen und Wirkungsbreite} der des SGB IX

Hinsichtlich der tatbestandlichen Voraussetzungen nennt das SGB IX die Behinderung; diese ist durch folgende Merkmale legal-definiert ${ }^{46}$ ): (1) Abweichung von einem für das Lebensalter typischen Zustand in - alternativ - dreierlei Hinsicht: (a) körperliche Funktion, (b) geistige Fähigkeit, (c) seelische Gesundheit; weiterhin (2) Dauer dieser Abweichung - prognostisch festgestellt: mit hoher Wahrscheinlichkeit - länger als sechs Monate; außerdem (3) Beeinträchtigung der Teilhabe am Leben in der Gesellschaft; schließlich muss (4) die Beeinträchtigung durch die Abweichung verursacht sein.

Wie bereits erwähnt, enthält das SGB IX ${ }^{47}$ ) einen weiteren Grundtatbestand; dieser unterscheidet sich in der normativen Ausgestaltung von der "Behinderung" lediglich dadurch, dass auch die Gefahr einer Behinderung legal definiert und somit normativ erfasst wird ${ }^{48}$.

Die damit definierte Wirkungsbreite der „Behinderung“ ist erläuterungsbedürftig; dazu gibt einen sicherlich verlässlichen, weil unmittelbar auf gesetzlicher Basis gewonnenen Aufschluss die Bestimmung über die „Leistungen zur Teilhabe“" $\$ 4$ Abs I SGB IX nennt explizit die Ziele des Gesetzgebers im Hinblick auf die gesetzlich vorgesehenen Interventionen bei Behinderung ${ }^{49}$ ); welche Leistungen im Einzelnen zu erbringen sind, wird - gegliedert in vier Leistungsgruppen ${ }^{50}$ ) -in den Kapiteln vier bis sieben SGB IX ${ }^{51}$ ) geregelt.

45) Ähnlich dem SGB IV in seiner Funktion als „Allgemeiner Teil“ des SV-Rechts.

$\left.{ }^{46}\right) \mathrm{Vgl}$ im Einzelnen $\$ 2$ Abs 1 S 1 SGB IX.

$\left.{ }^{47}\right)$ In $\$ 2 \mathrm{Abs} 1 \mathrm{~S} 2$.

${ }_{48}$ Im Nachfolgenden soll dieser Tatbestand nicht stets und zugleich gedanklich mitgeführt oder mitverfolgt werden.

${ }^{49}$ ) Das folgt auch aus $₫ 4$ Abs 2 S 1 SGB IX, der die Einschätzung ausdrücklich bestätigt.

$\left.{ }^{50}\right)$ Siehe $\$ 5$ SGB IX.

$\left.{ }^{51}\right) \$ 26 \mathrm{ff}, 33 \mathrm{ff}, 44 \mathrm{ff}, 55 \mathrm{ff}$ SGB IX.
Der Rückschluss aus den Zielsetzungen des SGB IX auf die Wirkungsbreider Behinderung angesichts der „Leistungen zur Teilhabe“ und diesbezüglich denkbare Beeinträchtigungen führen zu folgender Erkenntnis: Bereits die Behinderung im Sinn des SGB IX (also ohne bereichsspezifische Konkretisierung) umfasst neben den in $\$ 2$ Abs 1 SGB IX beschriebenen Defiziten auch Beeinträchtigungen der Erwerbsfähigkeit. Sie liegt zudem vor bei Pflegebedürftigkeit, wie aus $\$ 4$ Abs $1 \mathrm{Nr} 2$ SGB IX zu entnehmen ist. Sie ist weiterhin in allen weiteren Fällen geren, in denen andere Sozialleistungen (als bei im weitesten Sinne verminderter Erwerbsfähigkeit und Pflegebedürftigkeit $\left.{ }^{52}\right)$ ) erbracht werden ${ }^{53}$ ).

Unter dem Gesichtspunkt der "Teilhabe am Arbeitsleben“ ist die Behinderung übrigens ebenfalls relevant insoweit, als die berufliche Tätigkeit (negativ) betroffen ist.

Insgesamt zeigt also der Blick auf das SGB IX einen tatbestandlich weiten Anwendungsbereich, der begrifflich zweistufig geregelt ist, nämlich (in der ersten Stufe) durch eine Definition bestimmter, dauerhafter Defizite und (in der zweiten Stufe) durch daraus sich ergebende ${ }^{54}$ ) Beeinträchtigungen.

Im Übrigen lassen sich zum näheren Verständnis sowohl der Defizite in der 1. Regelungsstufe als auch der Beeinträchtigungen in der 2. (Tatbestands-)Regelungsstufe die Regelungen heranziehen, die Näheres zu den Leistungen innerhalb der Leistungsgruppen aussagen.

Der Vergleich mit dem Rehabilitationsrecht des SGB VI soll nunmehr Gemeinsamkeiten und Unterschiede aufdecken.

\subsection{Die rehabilitationsrechtlichen Regelungen des SGB VI}

Das Rentenversicherungsrecht bezeichnet ausdrücklich (auch) die Leistungen der medizinischen Rehabilitation als „Leistungen zur Teilhabe $\left.{ }^{(55}\right)$. Bei der Suche nach dem gesetzlichen (Grund-)Tatbestand des Rentenversicherungsrechts, bei dessen Vorliegen diese Leistungen zu gewähren sind, zeigt sich eine erste Schwierigkeit. Wie bereits erwähnt, fehlt eine ausdrückliche griffige $\mathrm{Be}-$ zeichnung wie zum Beispiel die Krankheit im SGB V; das macht allerdings die Suche nach dem gesetzestechnisch notwendigen Tatbestand nicht entbehrlich, im Übrigen auch nicht unmöglich.

Auch hier kann die teleologische Betrachtungsweise praktiziert werden dergestalt, dass die Zielsetzungen sowie die ausdrücklichen Leistungskataloge an im Gesetz festgelegten Leistungen als Auslegungshilfe herangezogen werden können bei der Frage, welche tatbestandsmäßigen Voraussetzungen dem Gesetzgeber vorgeschwebt haben müssen.

${ }^{2}$ ) Dies wohl iSd SGB XI, nach den dortigen dezidiert normierten Voraussetzun gen von „Pflegebedürftigkeit“.

53) Vgl $\$ 4$ Abs 1 Nr 2 SGB IX. dert.

$\left.{ }^{54}\right)$ Die kausale Verknüpfung beider Stufen wird durch das Wort „daher" gefor-

$\left.{ }^{55}\right)$ Siehe $\$ \$ 9,10-12 ; 13 ; 15 ; 20 ; 31 ; 32$ SGB VI. 


\subsubsection{Die rentenrechtlichen "Grundtatbestände"}

Man wird in auf diesem Weg in der Tat fündig, und zwar zunächst in der gesetzgeberischen Festlegung der "Aufgabe der Leistungen zur Teilhabe“ im SGB VI ${ }^{56}$ ). Daraus ist zu entnehmen, dass diese Leistungen zu erbringen sind bei „Auswirkungen“, die entweder auf einer Krankheit oder auf einer (körperlichen, "geistigen oder seelischen) Behinderung beruhen; damit nimmt das SGB VI of fenbar Bezug sowohl auf den zentralen Versicherungsfall des SGB V, die „Krankheit" als auch auf den Grundtatbestand des SGB IX, die „Behinderung".

Fraglich ist, welcher Zustand - oder welche Zustände, also tatsächliche Gegebenheiten - damit beschrieben werden. Dem Wortlaut nach soll es offenbar nicht um Zustände gehen, die identisch sind mit „Krankheit" oder „Behinderung“, obwohl man auf den ersten Blick meinen könnte, dass sowohl die als Krankheit oder Behinderung definierten Gegebenheiten jeweils identisch sind mit deren Auswirkungen.

Die Antwort auf diese Frage steckt im Gesetz selbst: Die Auswirkungen von Krankheit oder Behinderung sind insoweit relevant, als die Erwerbsfähigkeit der Versicherten betroffen ist. Dabei ist es selbstverständlich, dass der Gesetzgeber nur negative Auswirkungen, also Beeinträchtigungen der Erwerbsfähigkeit, meint; dies folgt auch aus den in $\$ 9$ Abs 1 Satz 1 Nr 2 SGB VI genannten Zielen der Leistungen zur medizinischen Rehabilitation.

Somit zeigt sich weiterhin, dass in $\$ 9$ Abs 1 Satz 1 Nr 1 SGB VI mehrere Tatbestände normiert sind, die dem Grunde nach - alternativ - Voraussetzung für Leistungen zur medizinischen Rehabilitation sind: Es müssen Defizite vorliegen, infolge entweder von Krankheit oder von Behinderung, mit jeweils spezifische Auswirkungen - nämlich solchen, die sich negativ auf die Erwerbsfähigkeit auswirken.

Damit zeigt sich, dass im Rentenversicherungsrecht zwei spezifische Grundtatbestände existieren, bei denen aus den denkmöglichen, vielfältigen Wirkungen von Krankheit und Behinderung diejenigen als leistungsbegründend normiert sind, die zu einer Beeinträchtigung der Erwerbsfähigkeit geführt haben.

\subsubsection{Drohende Auswirkungen auf die Erwerbsfähigkeit}

Doch damit nicht genug. Dass diese Beeinträchtigungen nicht bereits vorliegen müssen, ergibt sich aus den Rehabilitationszielen des $\$ 9$ Abs 1 S 1 Nr 2 SGB VI; denn danach sind Rehabilitationsleistungen auch (und im Gesetz an erster Stelle genannt) zu erbringen, wenn Beeinträchtigungen der Erwerbsfähigkeit noch „zu verhindern" sind, also offensichtlich tatsächlich noch nicht gegeben sein müssen. Somit ist der Sache nach auch bereits eine Gefahr für den Erhalt der Erwerbsfähigkeit leistungsauslösend.

Gleiches gilt in Bezug auf eine Behinderung, die tatsächlich vorliegen muss $^{57}$ ), die aber ebenfalls noch nicht zu einer Beeinträchtigung der Erwerbsfähigkeit geführt haben muss.

${ }^{56)}$ \$9 SGB VI.

$\left.{ }^{57}\right)$ Eine drohende Behinderung; vgl $\$ 1, \$ 2$ Abs 1 Satz 2 SGB IX, reicht also nicht aus.
Im Ergebnis sind es demnach vier tatbestandsmäßig und gedanklich unterscidbare Fallgruppen, die zu Leistungen der medizinischen Rehabilitation im Rentenversicherungsrecht führen können ${ }^{58}$ ).

\subsubsection{Gemeinsames Merkmal der "Erheblichkeit"}

Die hiermit vorgenommene tatbestandsmäßige Analyse des rentenversicherungsrechtlichen Rehabilitationsrechts ${ }^{59}$ ) muss noch durch die Einbeziehung der persönlichen Voraussetzungen ${ }^{160}$ ) ergänzt werden. Ausweislich des Gesetzeswortlauts folgt aus $\$ 10$ Abs 1 Nr 1 SGB VI, dass bei den soeben dargestellten Tatbeständen, die eine Gefahr für die Beeinträchtigung der Erwerbsfähigkeit genügen lassen, stets ein gewisses Gefahrenpotential gegeben sein muss; denn die Erwerbsfähigkeit muss „erheblich gefährdet“ $\operatorname{sein}^{61}$ ).

Hinsichtlich der Tatbestände mit bereits eingetretener Minderung der Erwerbsfähigkeit sieht das Gesetz offenbar ebenfalls eine Differenzierung bei der Tatbestandsmäßigkeit vor, nämlich zwischen "geminderter Erwerbsfähigkeit und , teilweiser Erwerbsminderung " ${ }^{\prime 62}$ ); die Unterscheidung bezieht Tatbestände ein, bei denen zwar eine die Rentenzahlung vermeidende wesentliche Besserung der Erwerbsfähigkeit nicht mehr erwartet werden kann, durch die Leistungen jedoch der bisherige Arbeitsplatz erhalten werden kann ${ }^{63}$ ).

\subsection{Tatbestandsbezogener Vergleich zwischen SGB VI und SGB IX}

Vergleicht man die tatbestandlichen Voraussetzungen des „allgemeinen Rehabilitationsrechts" des SGB IX mit den entsprechenden bereichsspezifischen Regelungen des SGB VI ${ }^{64}$ ), so lässt sich (im Hinblick auf das Merkmal der „Behinderung") unschwer Folgendes feststellen: Im Recht des SGB IX muss eine Behinderung nicht vorliegen; es reicht vielmehr auch eine drohende Behinderung, also die Gefahr einer solchen „Abweichung" aus ${ }^{65}$ ).

Demgegenüber muss im SGB VI eine Behinderung vorliegen; diese kann auf körperliche, geistige oder seelische Defizite zurückzuführen sein - insoweit besteht Übereinstimmung mit dem allgemeinen Rehabilitationsrecht, demzufolge Ursache für die Behinderung - und auch für die drohende Behinderung - Abweichungen sowohl im Hinblick auf die körperlichen Funktionen als auch auf die geistigen Fähigkeiten und die seelische Gesundheit sein können ${ }^{66}$ ).

$\left.{ }^{58}\right)$ Für einen „real existierenden“ Anspruch sind die weiteren „persönlichen“ sowie versicherungsrechtlichen Vorraussetzungen“ gem $\$ \$ 10,11$ SGB VI erforderlich.

$\left.{ }^{59}\right)$ Wiederum nur hinsichtlich der medizinischen Rehabilitation.

$\left.{ }^{60}\right) \$ 10$ SGB VI.

${ }^{61)} \$ 10 \mathrm{Abs} 1 \mathrm{Nr} 1$ - siehe auch $\mathrm{Nr} 2-\mathrm{SGB}$ VI.

$\left.{ }^{62}\right) \$ 10 \mathrm{Abs} 1 \mathrm{Nr} 2$, einerseits lit a, andererseits lit b SGB VI.

${ }^{63}$ ) Niesel in Kasseler Kommentar SV-Recht (KassKomm-Niesel), $\$ 10$ SGB VI Rn 12

${ }^{64)}$ Wiederum nur auf die medizinische Rehabilitation bezogen

$\left.{ }^{65}\right) \$ 1$ S $1, \$ 2$ Abs 1 S 2 SGB IX

$\left.{ }^{66}\right) \$ 2$ Abs 1 S 1 SGB IX. 


\subsubsection{Die Bedeutung der Krankheit}

Im Rehabilitationsrecht des SGB VI ist ausdrücklich auch die Krankheit mit ihren negativen Auswirkungen auf die Erwerbsfähigkeit als leistungsbegründender Tatbestand genannt. Diese Voraussetzung steht - im SGB VI - selbstständig neben der Behinderung, und zwar ebenfalls mit ihren rentenrechtlich spezifischen Auswirkungen, die aus einer erheblichen Beeinträchtigung der Erwerbsfähigkeit (oder aus einer drohenden erheblichen Beeinträchtigung der Erwerbsfähigkeit) bestehen müssen.

Darüberhinaus erweitert das SGB VI - im Vergleich mit dem SGB IX - den Anwendungsbereich des Rehabilitationsrechts aus tatbestandsmäßiger Betrachtung in folgender Weise: Während gem $\$ 2$ Abs 2 Satz 1 SGB IX eine Behinderung nur vorliegt, wenn die Abweichung mit hoher Wahrscheinlichkeit länger als sechs Monate dauert ${ }^{67}$ ) oder die drohende Behinderung voraussichtlich ebenfalls zumindest für diesen Zeitraum andauern wird ${ }^{68}$ ), ist dem Rentenversicherungsrecht dieses einschränkende Tatbestandsmerkmal in Bezug auf die Krankheit (im Sinne einer Relevanz nur dann, wenn ein bestimmter Zeitraum des defizitären Zustands gegeben ist oder zu erwarten ist) nicht beigegeben; damit stimmt dieser Krankheitsbegriff des SGB VI mit dem des KV-Rechts überein ${ }^{69}$ ).

Daraus folgt, dass Leistungen der medizinischen Rehabilitation auch ohne die Notwendigkeit der typischen Voraussetzungen des allgemeinen Rehabilitationsrechts des SGB IX zu erbringen sind, also insb auch ohne die tatbestandmäßig vorauszusetzende „Sechs-Monats-Prognose“.

\subsubsection{Die Bedeutung der Behinderung}

Diese Feststellung führt zunächst zu folgender Frage: Kommt der „Behinderung" mit den rentenrechtlich typischen Auswirkungen überhaupt eine Bedeutung $\mathrm{zu}$ angesichts des umfassenden Tatbestandes der „Krankheit“, die ebenfalls „nur" hinsichtlich der rentenrechtlichen Auswirkungen beachtlich ist? Es schein - auf den ersten Blick - so zu sein, dass der „Krankheits-Grundtatbestand“ den „Behinderungs-Grundtatbestand“ konsumiert, zumal die rechtlich relevanten Ursachen von Behinderung ${ }^{70}$ ) mit denen der „Krankheit“ anscheinend übereinstimmen $^{71}$ ).

Wenn diese Vermutung zuträfe, wäre die Behinderung (sowie wohl auch die drohende Behinderung) als tatbestandliche Grundvoraussetzung im rentenversicherungsrechtlichen Rehabilitationsrecht überflüssig.

Bei der Antwort auf diese Frage ist allerdings zu berücksichtigen, dass Behinderung und Krankheit zwar über gemeinsame Begriffsmerkmale verfügen ${ }^{72}$ ),

${ }^{67)}$ Hierzu Welti, aaO $\$ 2$ Rn 26.

$\left.{ }^{68}\right)$ Dies ergibt sich letztlich aus dem Vorrang der Prävention gem $\$ 3$ SGB IX, sa Welti, aaO $\$ 2$ Rn $32-36$

${ }^{69)}$ Ebenso KassKomm-Niesel, $\$ 10$ SGB VI Rn 4 mwN

$\left.{ }^{70}\right)$ S $\$ 2$ Abs 2 S 1 SGB IX; siehe auch $\$ 9$ Abs 1 S 1 Nr $1, \$ 10$ Abs 1 Nr 1 SGB VI.

$\left.{ }^{71}\right) \mathrm{Vgl} \$ 2$ Abs $1 \mathrm{~S} 1 \mathrm{SGB}$ IX sowie allgemein zu den Voraussetzungen von Krankheit KassKomm-Höfler, $\$ 27 \mathrm{Rn} 9 \mathrm{ff}$

72) Vgl oben 2.1. dass aber auch ein sowohl rechtlich als auch tatsächlich vorhandener wesentlicher Unterschied zwischen beiden Zuständen besteht: definitionsgemäß, zumindest ihrem Wesen nach und anknüpfend an das überkommene Verständnis, betrifft "Rehabilitation" typischerweise die Situation, in der die "Akutversorgung" eines "Kranken abgeschlossen ist ${ }^{73}$ ), jedoch gegenüber einem (im Einzelfall festzustellenden) optimalen Gesundheitszustand noch Defizite bestehen, die an sich beseitigt werden können.

Soweit erfasst die Behinderung (und deren Auswirkungen) zumindest gesetzestechnisch und gedanklich eine andere Situation als diejenige, in die durch "Krankheit" definiert ist.

Eine weitere Vermutung liegt nahe: Wenn im SGB VI auch die Krankheit als leistungsbegründender Tatbestand ausreicht ${ }^{74}$ ), dann stellt sich die Frage sowoh nach der Sinnhaftigkeit des gesamten Rechts der Rentenversicherung in Bezug auf seine Leistungen der medizinischen Rehabilitation; denn grundsätzlich ist für die Krankenbehandlung die GKV zuständig. Und deren Krankheits-Grundtatbestand umfasst an sich, insb auch aus medizinisch-fachlicher Betrachtungsweise, auch die rentenversicherungsrechtlich typischen Fälle mit ihren speziellen Krankheitsauswirkungen.

Man kann diese Frage gleichwohl zustimmend beantworten dahingehend dass dieses Krankheitssegment einen besonderen Bezug zum „Kerngeschäft“ des Rentenversicherungsrechts hat und die Anordnung "Rehabilitation vor Rente" - genau müsste man heutzutage sagen „Krankenbehandlung sowie Rehabilitation vor Rente ${ }^{\text {"75 }}$ ) - dort „,in den besseren Händen“ ist.

Gleichwohl liegt das praktische Problem des Gesetzesvollzuges auf der Hand: Bei einer derart aufgeteilten Zuständigkeit verschiedener Versicherungsbereiche für die Krankenbehandlung ist ein Problem ersichtlich „vorprogrammiert"; es besteht in der Schwierigkeit der primär medizinischen Frage (und der daraus sich ergebenden juristischen Zuordnung), ein Krankheitsgeschehen im Hinblick auf die spezifisch rentenrechtlich relevanten Wirkungen eindeutig und in $\mathrm{Ab}$ grenzung zu den Leistungen der KV exklusiv dem Bereich der rentenrechtlichen Rehabilitation zuzuordnen.

\subsection{Die Leistungen der medizinischen Rehabilitation.} Vergleich zwischen SGB IX und SGB VI

Die Darstellung des Rechts der medizinischen Rehabilitation muss auch die dort geregelten Leistungen betrachten; auch dabei richtet sich der insoweit vergleichende Blick auf die Bereiche, in denen diese Leistungen gewährt werden. $\mathrm{Zu}$ bedenken ist dabei auch, dass die Zielsetzungen, die in den Regelungen zu

${ }^{73}$ ) Und damit nach traditioneller Sicht auch die Leistungspflicht der gesetzlichen $\mathrm{KV}$ endet.

${ }^{74}$ ) Auch diesbezüglich lediglich relevant hinsichtlich der bereichsspezifischen Auswirkungen des Rentenversicherungsrechts (erhebliche Beeinträchtigung der Erwerbsfähigkeit, alternativ die Gefahr einer solchen Beeinträchtigung).

${ }^{75}$ ) Um damit auch terminologisch die Anbindung von Rehabilitationsleistungen an das Vorliegen einer (drohenden) Behinderung zu vermeiden. 
Inhalt und Umfang der Rehabilitationsleistungen enthalten sind, zugleich auch die Anspruchsvoraussetzungen und dabei durchaus auch die im Einzelfall gegebenen tatbestandlichen Voraussetzungen mitbestimmen; auch insoweit ist der Vergleich des allgemeinen Rehabilitationsrechts das SGB IX mit den verschiedenen rehabilitationsrechtlichen Regelungen in den Besonderen Teilen des SGB aufschlussreich.

\subsubsection{Rehabilitationsleistungen nach dem SGB IX}

Zum Verhältnis der rehabilitationsrechtlichen Regelungen zwischen dem SGB IX des und dem SGB VI zueinander ist allgemein folgendes vorweg zu bemerken: Die Leistungen zur medizinischen Rehabilitation sind in den $\$ \$ 26$ bis 31 SGB IX geregelt. Das SGB VI nimmt auf diese Vorschriften in $\$ 15$ Abs 1 SGB VI zunächst ausdrücklich Bezug, mit (verständlicherweise) ausdrücklicher Ausnahme der Leistungen zur Früherkennung und Frühförderung behinderter und von Behinderung bedrohter Kinder ${ }^{76}$ ); weiterhin konkretisiert das SGB VI den Leistungsumfang ${ }^{77}$ ), wobei dem zeitlichen Aspekt der nach dem SGB VI gewährte Leistungen zur medizinischen Rehabilitation besondere Bedeutung zukommt.

\subsubsection{Die Regelungstechnik des SGB IX}

Der SGB IX - Gesetzgeber benutzt bei der Normierung der Leistungen folgende Regelungstechnik: In $\$ 26$ Abs 1 SGB IX werden Ziele genannt, die in zwei Gruppen aufgeteilt sind. Die erste Gruppe (in $\$ 26$ Abs 1 Nr 1 SGB IX aufgeführt) befasst sich mit Behinderungen „einschließlich chronischer Krankheiten“; damit sind bereits zwei Zielgruppen erfasst, nämlich - erstens - diejenigen Personen, bei denen die gesetzlichen Merkmale des $\$ 2$ Abs 1 Satz 1 SGB IX („,Behinderung“) vorliegen und - zweitens - Personen mit bestimmten, nämlich chronischen Krankheiten. Da die zweite Personengruppe (der chronisch Kranken) in die Gruppe der Behinderten „eingeschlossen“ ist, handelt es sich um eine Teilmenge von behinderten Menschen ${ }^{78}$ ). So gesehen wird der das SGB IX prägende "Kreis der Behinderten“ nicht erweitert.

Bemerkenswert ist allerdings, dass damit möglicherweise nicht diejenigen chronisch kranken Menschen erfasst werden, deren Krankheitsgeschehen und -behandlung noch nicht abgeschlossen ist, die sich also noch in der Akutbehandlung befinden und bei denen ein Urteil über das Vorliegen einer Behinderung (oder einer drohenden Behinderung) noch nicht getroffen werden kann. Bei diesem Personenkreis liegen die Merkmale von „Behinderung“ demnach nicht vor; somit ist nicht ausgeschlossen, dass damit ein Teil von chronisch Kranken (ohne

${ }^{76)} \$ 26 \mathrm{Abs} 2 \mathrm{Nr} 2$ sowie $\$ 30$ SGB IX

${ }^{77)}$ Bei stationärer Rehabilitation im Hinblick auf einen Zeitrahmen $(\$ 15$ Abs 3 SGB VI), allgemein mit Hinweis auf die Grundsätze von Wirtschaftlichkeit und Sparsamkeit ( $\$ 13$ Abs 1 SGB VI).

${ }^{78}$ ) Damit folgt der Gesetzgeber der Begrifflichkeit der WHO, mit der der steigenden Zahl chronisch kranker Menschen in besonderer Weise Rechnung getragen werden soll, vgl hierzu Welti, aaO $\$ 26 \mathrm{Rn} 11$; zur Orientierung des Gesetzgebers an den Vorstellungen der WHO allgemein Welti, aaO Einführung Ziff $7.2,14 \mathrm{ff}$.
Behinderung oder ohne drohende Behinderung) von den Regelungen zur medizinischen Rehabilitation nicht erfasst wird, während die chronisch Kranken mit Behinderung dem Rehabilitations-Leistungsrecht unterfallen.

\subsubsection{Weitere Zielgruppen}

$\$ 26$ Abs 1 Nr 2 SGB IX enthält alternativ ${ }^{79}$ ) zu Abs 1 Nr 1 zwei weitere Zielgruppen: Genannt werden „Einschränkungen der Erwerbsfähigkeit“ und „Pflegebedürftigkeit" - hinsichtlich beider Sachverhalte geht es gleichermaßen um Vermeidung, Überwindung, Minderung sowie der Verhütung von Verschlimmerung.

Zusätzlich werden die Ziele „Vermeidung von vorzeitigem Bezug von laufenden Sozialleistungen“ oder - alternativ - „Minderung laufender Sozialleistungen" genannt; man darf wohl annehmen, dass damit nicht zusätzliche Sachverhalte und Personengruppen angesprochen werden, sondern dass es sich dabei um gleichsam parallele Erscheinungen handelt, die mit den Zielen im Hinblick auf Erwerbsfähigkeit und Pflegebedürftigkeit in der Regel zeitgleich einhergehen.

Bemerkenswert ist im Hinblick auf $\$ 26$ Abs 1 Nr 2 SGB IX, dass die beiden dort erfassten Fallgruppen nicht ausdrücklich das Merkmal der „Behinderung aufweisen; damit scheint der Anwendungsbereich der Leistungen zur medizinischen Rehabilitation über das Kerngebiet des Rehabilitationsrechts hinaus ausgedehnt worden zu sein, wenn man das traditionelle Verständnis dieses $\mathrm{Be}$ griff $^{80}$ ) zugrundelegt.

Diese Sichtweise ist jedoch nicht zutreffend. Ein derart restriktives Verständnis, dass nämlich Einschränkungen der Erwerbsfähigkeit sowie Pflegebedürftigkeit nur insoweit relevant sind, als diese Tatbestände bei Behinderten (oder bei Menschen, die von Behinderung bedroht sind) auftreten, folgt aus $\$ 26$ Abs $1^{81}$ ) SGB IX. Somit führt zum Beispiel Pflegebedürftigkeit nur dann zu den Leistungen zur medizinischen Rehabilitation gem $\$ 26$ SGB IX, wenn zugleich eine Behinderung vorliegt (oder droht); um dies festzustellen ist auf $\$ 2$ Abs 1 SGB IX zurückzugreifen.

\subsubsection{Behandlungen}

Bei $\$ 26$ Abs 1 SGB IX ist hinsichtlich der darin enthaltenen gesamten Aussage schließlich noch festzuhalten, dass in diesen Zielsetzungen ${ }^{82}$ ) auch Tätigkeitsfelder stecken, auf denen im Wesentlichen wohl „Behandlungen“ von Menschen vorgenommen werden; zum Beispiel ist die Abwendung von Behinderung oder chronischer Krankheiten oder die Vermeidung der Einschränkung der Erwerbs-

\footnotetext{
$\left.{ }^{79}\right)$ Das ergibt sich eindeutig aus dem Wortlaut: „oder“ in $\$ 26 \mathrm{Abs} 1 \mathrm{Nr} 1 \mathrm{aE}$

$\left.{ }^{80}\right)$ Siehe oben 2.

${ }^{81}$ ) Vor Nr 1.

${ }^{82}$ ) So auch die eigene Einschätzung des Gesetzgebers in $\$ 27$ SGB IX
} SGB IX. 
fähigkeit notwendigerweise mit Tätigkeiten verbunden, die je nach Sichtweise als "Leistungen ${ }^{\text {"83 }}$ ) oder als „Behandlungen ${ }^{\text {"84 }}$ ) zu bezeichnen sind.

Näheres hierzu regelt der Gesetzgeber in $\$ 26$ Abs 2 SGB IX. Hier wird die Leistungserbringung beispielhaft ${ }^{85}$ ) angesprochen; genannt werden die Leistungserbringer ${ }^{86}$ ), einzelne Behandlungsmethoden ${ }^{87}$ ) sowie die Zuwendung von Arznei-, Verbands- und Hilfsmitte ${ }^{88}$ ).

Auch diese Regelungen lassen Rückschlüsse zu über Art und Umfang der vom Gesetzgeber vorgesehenen Interventionen bei gesundheitlichen Defiziten.

\subsubsection{Annex-Leistungen“}

Der Leistungskatalog wird in $\$ 26$ Abs 3 SGB IX durch so genannte AnnexLeistungen erweitert, und zwar um „medizinische, psychologische und pädagogische Hilfen"; beispielhaft werden auch diesbezüglich die wohl wichtigsten Leistungsbereiche genannt. Dass diese Leistungen nur „im Einzelfall“ gewährt werden und nur dann, wenn sie zur Erreichung der Rehabilitationsziele „erforderlich" sind, hat keinen konstitutiven Regelungsgehalt; diese Aussage kann nur als gesetzgeberischer Hinweis auf etwas Selbstverständliches verstanden werden.

Da diese Leistungen „Bestandteil der Leistungen“ nach $\$ 26$ Abs 1 SGB IX sind $\left.^{89}\right)$, ist ihre Gewährung auf die Dauer der Leistungen gem $\$ 26$ Abs 1 SGB IX beschränkt ${ }^{90}$ ). Sind diese Leistungen - zum Beispiel medizinische Hilfen zur seelischen Stabilisierung - über diesen Zeitraum hinaus erforderlich, kommt $\$ 55$ Abs 1 SGB IX in Betracht ${ }^{91}$ ). Allerdings bedarf es einer leistungsrechtlichen Anspruchsgrundlage in einem der Besonderen Teile des SGB, die den $\$ 55$ Abs 1 SGB IX in ihren Regelungsbereich einbezieht; es handelt sich um Teilhabe-Leistungen der Leistungsgruppe $\mathrm{Nr} 4$ (Leistungen zur Teilhabe am Leben in der Gemeinschaft ${ }^{92}$ ), die gem $\$ 6$ Abs 1 SGB IX unter anderem von den Trägern der gesetzlichen $\mathrm{UV}^{93}$ ) und der Sozialhilfe ${ }^{94}$ ), nicht jedoch von der gesetzlichen KV, Rentenversicherung oder Bundesagentur für Arbeit erbracht werden ${ }^{95}$ ).

83) So die rechtliche Betrachtung und die Bezeichnung in $\$ 26$ Abs 3 SGB IX

${ }^{84)}$ So eher die tatsächliche Vorgehensweise bei der Leistungserbringung. Rn 14.

(ie sich aus „insb“ ergibt; vgl dazu auch Stähler, aaO $\$ 26$

${ }^{86)} \$ 26$ Abs 2 Nr 1 SGB IX.

${ }^{87)} \$ 26$ Abs $2 \mathrm{Nr} 2,4,5,7$ SGB IX

$\left.{ }^{88}\right) \$ 26$ Abs $2 \mathrm{Nr} 3,6$ SGB IX.

$\left.{ }^{89}\right)$ Daher die Bezeichnung als „Annex-Leistungen“.

${ }^{90}$ ) Ebenso Stähler, $\mathrm{aaO} \$ 27 \mathrm{Rn} 22$

9) Im Rahmen der Leistungen zur „Teilhabe am Leben in der Gemeinschaft“, vgl $\$ \$ 5 \mathrm{Nr} 4,55 \mathrm{ff}$ SGB IX.

${ }^{92}$ ) Siehe $\$ \$ 5 \mathrm{Nr} 4,55 \mathrm{ff}$ SGB IX.

93) Gem $\$ 27$ Abs 1 Nr 7 SGB VII iVm mit $\$ 26$ Abs 3 SGB IX.

$\left.{ }^{94}\right)$ Gem $\$ 8$ Abs 2 SGB XII Eingliederungshilfe; zur Eingliederungshilfe für behinderte Personen vgl $\$ 53$ Abs 1 SGB XII.

${ }^{95}$ ) Weitere Träger der Leistungen gem $\$ 5 \mathrm{Nr} 4$ SGB IX sind die Träger der Kriegsopferversorgung und der Kriegsopferfürsorge und die Träger der öffentlichen Jugendhilfe, $\operatorname{vgl} \$ 6$ Abs $1 \mathrm{Nr} 5$ und 6 SGB IX.
4.7.6 Leistungen zur „stufenlosen Wiedereingliederung“

Die leistungsrechtlichen Regelungen zur medizinischen Rehabilitation des SGB IX nennen weiterhin die "stufenweise Wiedereingliederung"; der Sache nach handelt es sich um Fälle der Teil-Arbeitsunfähigkeit ${ }^{96}$ ).

Medizinische und ergänzende Leistungen der medizinischen Rehabilitation sollen entsprechend eingesetzt werden, wobei es sich nicht um Maßnahmen der Belastungserprobung, Arbeitstherapie, Berufsfindung oder Arbeitserprobung handelt; vielmehr knüpfen die Leistungen an betriebsbezogene Maßnahmen mit rehabilitiver Zielsetzung $\mathbf{a n}^{97}$ ).

\subsubsection{Förderung der Selbsthilfe}

Außerdem wird der Leistungskatalog des $\$ 26$ Abs 2 SGB IX durch die Regelung in $\$ 29$ SGB IX zur „Förderung der Selbsthilfe “ konkretisiert ${ }^{98}$ ).

\subsubsection{Allgemeine Regelungen des SGB IX für die Leistungen zur} medizinischen Rehabilitation

Die Wirkung der materiell-rechtlichen Regelungen zur medizinischen Rehabilitation wird nicht nur durch die speziellen Vorschriften des SGB IX ${ }^{99}$ ), sondern auch durch die „allgemeinen Regelungen“ des SGB IX ${ }^{100}$ ) insoweit bestimmt, als diese Vorschriften einen materiell-rechtlichen Gehalt aufweisen, also bei der Frage nach dem Bestehen eines Anspruchs und dessen Ausgestaltung im Einzelnen heranzuziehen sind.

Dass eine derartige definitorisch - systembildende Funktion von der Legaldefinition von „Behinderung " und „drohender Behinderung“ ( $\$ 2$ Abs 1 SGB IX) ${ }^{101}$ ) sowie von den allgemeinen Zielsetzungen des SGB IX ausgehen könnte (die in $\$ 26$ Abs 1 SGB IX ebenfalls und zudem spezifiziert aufgeführt sind), wurde bereits erwähnt ${ }^{102}$ ).

\subsubsection{1 „Prävention und Rehabilitation vor Rente“}

Objektivrechtliche Bedeutung hat möglicherweise die Verpflichtung der Rehabilitationsträger darauf hinzuwirken, dass der Eintritt einer Behinderung ein-

$\left.{ }^{96}\right)$ Ausdrücklich kennt das SV-Recht diesen Tatbestand nicht, ebenso Stähler, aaO $\S 28 \mathrm{Rn} 13 \mathrm{mwN}$.

) Stähler, aaO $\$ 28 \mathrm{Rn} 3$.

$\left.{ }^{98}\right) \$ 29$ - Förderung der Selbsthilfe, Vgl $\$ 26$ Abs 1 Nr 1 SGB IX: „... eigene Heilungskräfte entwickeln ...", Abs $3 \mathrm{Nr}$ 2: „Aktivierung von Selbsthilfepotentialen", $\mathrm{Nr} 5$, 6: „Training sozialer, kommunikativer und lebenspraktischer Fähigkeiten“; $\$ 30$ - Früherkennung und Frühförderung, vgl $\$ 26$ Abs 2 Nr 2 SGB IX, sowie eine diesbezügliche Verordnungsermächtigung in $\$ 32 \mathrm{Nr} 1$ SGB IX; $\$ 31$ - Hilfsmittel, vgl $\$ 26 \mathrm{Abs} 2 \mathrm{Nr} 6$ SGB IX, sowie eine diesbezügliche Verordnungsermächtigung in $\$ 32 \mathrm{Nr} 2$ SGB IX.

$\left.{ }^{99}\right) \$ \$ 26-32$ SGB IX

$\left.{ }^{100}\right)$ 1. Kapitel, $\$ \$ 1-16$ SGB IX

${ }^{101}$ \$ 2 Abs 1 SGB IX; die "Schwerbehinderung“ - vgl \$ 2 Abs 2, 3 SGB IX hat Bedeutung für das 2. Kapitel des SGB IX, in dem das Schwerbehindertenrecht geregelt ist.

102) Vgl oben bei 3 . 
schließlich einer chronischen Krankheit vermieden wird und vom Gesetzgebe ls "Vorrang von Prävention " betitelt wird ${ }^{103}$ ); jedenfalls bei Ermessensentscheidungen wird diese Pflicht zu berücksichtigen sein. Zudem ist diese Reglung eine Auslegungshilfe bei der Festlegung von Leistungen auch dort, wo Ermessen nicht eingeräumt ist oder ein Beurteilungsspielraum nicht besteht.

Weiterhin ist zu diesem traditionellen Grundsatz folgendes zu bemerken: zwar steckt in dem in $\$ 8$ Abs 2 Satz 1 SGB IX normierten Grundsatz „Reha vor Rente" ein materiell-rechtlicher Gehalt ${ }^{104}$ ); dieser betrifft allerdings nur eine spezielle Situation, in der einerseits die gesetzlichen Voraussetzungen für eine Rente $^{105}$ ) vorliegen, andererseits aber zugleich ein Anspruch auf Rehabilitationsleistungen gegeben ist. In diesem Fall wird dieser Anspruch dahingehend erweitert, oder - mit anderen Worten - gefestigt, dass der Leistungsträger trotz der Möglichkeit zur Gewährung von Rente die Voraussetzungen von Rehabilitationsleistungen einschließlich deren (voraussichtlich) dauerhafter Erfolge prüfen und gegebenenfalls dem Versicherten anbieten muss ${ }^{106}$ ).

\subsubsection{Wunsch- und Wahlrecht}

Schließlich hat das „Wunsch- und Wahlrecht des Leistungsberechtigten“ gem $\$ 9$ SGB IX ${ }^{107}$ ) materiell-rechtliche Bedeutung. Diese Norm wird nicht verdrängt durch Vorschriften, in denen dem Rehabilitationsträger Ermessen über Art, Dauer, Umfang, Beginn und Durchführung der Leistungen oder über die Rehabilitationseinrichtung eingeräumt wird ${ }^{108}$ ); vielmehr ist $\$ 9$ SGB IX ${ }^{109}$ ) im Rahmen der Ermessensausübung zu beachten ${ }^{110}$ ). Allerdings ist auch zu beachten, dass der nahezu selbstverständliche Hinweis des Gesetzgebers, dass nur geeignete Maßnahmen gefordert und geleistet werden dürfen ${ }^{111}$ ) - und insoweit stimmen SGB IX SGB VI überein -, durch das Wunsch- und Wahlrecht nicht überwunden werden kann.

\subsubsection{Regelungen des SGB IX mit eigenständiger Bedeutung}

Nach den bisherigen Bemerkungen zum Regelungsgehalt des SGB IX, im vorangegangenen überwiegend dargestellt auch im Vergleich zu den Bestimmungen des SGB VI zur medizinischen Rehabilitation, stellt sich die Frage nach eigenständigen Regelungen des SGB IX, die nicht der Sache nach durch spezielle

${ }^{103}$ \3 SGB IX; zu der verfahrensrechtlichen Folgerung iSd Verdrängung des Antragsprinzips vgl Welti, aaO $\$ 3 \mathrm{Rn} 7$.

$\left.{ }^{104}\right)$ Ebenso Welti, aaO $\$ 8 \mathrm{Rn} 23$.

$\left.{ }^{105}\right)$ Dazu Welti, aaO Rn 7 ff.

$\left.{ }^{106}\right)$ Diesen trifft im Fall eines solchen Angebots freilich nur eine Obliegenheit, so 源 en fehlender Mitwirkung“), 67 („Nachholen der Mitwirkung“) SGB I.

${ }^{107}$ ) Es handelt sich hierbei um eine Konkretisierung des $\$ 33$ S 2 SGB I.

$\left.{ }^{108}\right) \S 40$ Abs 3 S I SGB V, $\$ 13$ Abs 1 SGB VI, $\$ 26$ Abs 5 SGB VII

$\left.{ }^{109}\right)$ Ebenso wie $\$ 33 \mathrm{SGB}$.

10) Welti, aaO $\$ 9 \mathrm{Rn} 11$

111) $\mathrm{Vgl} \$ \$ 4,8$ Abs 1 SGB IX, $\$ 15$ Abs 1, 2 SGB VI.
Bestimmungen in einem der besonderen Teile insb des SV-Rechts der Sache nach beseitigt" werden.

Soweit ersichtlich, hat die höchstrichterliche Rechtsprechung nur in einem Fall eine solche eigenständige Bedeutung festgestellt. Es ging dabei um die Frage eines Hilfsmittels für eine schwer behinderte Frau und Mutter kleiner Kinder Den Anspruch auf eine relativ kostspielige Beinprothese für diese Frau hatte der KV-Träger abgelehnt mit Hinweis - im Wesentlichen - auf das Wirtschaftlichkeitsprinzip.

Das BSG hat der Versicherten ihren Anspruch zugesprochen und sich dabei auf eine „Allgemeine Regelung“ des SGB IX bezogen, der zufolge den „besonderen Bedürfnissen behinderter und von Behinderung bedrohter Frauen ... Rechnung getragen " werden muss ${ }^{112}$ ).

\subsection{Gestaltungsfreiheit der Verwaltung (Ermessen Beurteilungsspielraum)}

Nach dem SGB VI ist die Gewährung sämtlicher "Leistungen zur Teilhabe“, also auch die der Leistungen zur medizinischen Rehabilitation, in das Ermessen der Verwaltung gestellt; lediglich auf ergänzende Leistungen im Sinne des $\$ 28$ SGB VI besteht ein Anspruch, wenn eine Rehabilitationsleistung als „Hauptleistung" zuerkannt worden ist.

Diese Leistungen „können erbracht werden“, wenn die persönlichen und versicherungsrechtlichen Voraussetzungen ${ }^{113}$ ) dafür erfüllt sind ${ }^{114}$ ).

Weiterhin ist ausdrücklich festgelegt, dass der Träger der Rentenversicherung die Leistungen sowie die Rehabilitationseinrichtung „nach pflichtgemäßem Ermessen " bestimmt ${ }^{115}$ ).

In Rechtsprechung und Schrifttum besteht darüber Einigkeit, dass über das Wie" der Rehabilitationsleistung (das heißt Art, Dauer, Beginn und Durchführung) dem Versicherten nur ein Anspruch auf pflichtgemäße Ermessensausübung zusteht ${ }^{116}$ ); in der Dogmatik des allgemeinen Verwaltungsrechts spricht man diesbezüglich von „Auswahlermessen“, während das „Entschließungsermessen" die Frage betrifft, ob die Verwaltung überhaupt tätig werden darf oder muss $\left.^{117}\right)$.

Kein Ermessen ist dem Rentenversicherungsträger bezüglich des „ob“ der Rehabilitationsleistung (der so genannten Eingangsprüfung) eingeräumt; der Rehabilitationsträger muss also eine Entscheidung treffen ${ }^{118}$ ).

${ }^{112}$ ) BSGE SozR $3-2500 \$ 33 \mathrm{Nr} 44$ („C-leg“), mit Hinweis auf \$ 1 S 2 SGB IX; vg auch BSGE 93, $183=$ SozR $3-2500 \$ 33 \mathrm{Nr} 8$; Höfler weist darauf hin, dass diese Ent scheidungen im Widerspruch stehen zu $\$ 33$ SGB V, der lediglich einen "Basisausgleich" zulässt: KassKomm-Höfler $\$ 33$ SGB V $32-47$, zu „Prothesen“

$\left.{ }^{113}\right) \mathrm{Vgl} \$ \$ 10,11$ SGB VI.

$\left.{ }^{114}\right) \$ 9$ Abs 2 SGB VI.

$\left.{ }^{115}\right) \$ 13$ Abs 1 S 1 SGB VI

116) Vgl im Einzelnen KassKomm-Niesel \$13 SGB VI Rn 7.

$\left.{ }^{117}\right)$ Vgl für alle Maurer, Allgemeines Verwaltungsrecht ${ }^{14}(2002) \$ 7 \mathrm{Rn} 7 \mathrm{f}$

18) BSGE Soz R 3-2600 \$ $10 \mathrm{Nr} 2 \mathrm{~S} 3 \mathrm{mwN}$ 
Im Vergleich mit dem SGB IX zeigt sich folgendes: Auch im „allgemeinen Rehabilitationsrecht“ des SGB IX steht die „Ausführung von Leistungen“ im Ermessen der Leistungsträger ${ }^{119}$ ). Und auch hinsichtlich der Frage, ob eine leistungsberechtigte Person ein persönliches Budget erhält, entscheidet der Rehabilitationsträger im pflichtgemäßen Ermessen, da sich $\$ 9$ Abs 1 SGB IX auch auf die Ausführung der Leistungen bezieht ${ }^{120}$ ).

4.9 Gesetzliche Beschränkungen des Leistungsumfanges /

\section{Rationierung von Leistungen zur medizinischen Rehabilitation} Leistungsumfang

Stationäre Leistungen zur medizinischen Rehabilitation sollen für längstens drei Wochen erbracht werden; sie können für einen längeren Zeitraum erbrach werden, wenn dies erforderlich ist, um das Rehabilitationsziel zu erreichen ${ }^{121}$ ).

Weiterhin sieht der Gesetzgeber vor, dass regelmäßig Leistungen zur medizinischen Rehabilitation der Rentenversicherung erst vier Jahre nach Durchführung solcher oder ähnlicher Leistungen wiederholt werden dürfen, sofern die Kosten für die vorherige Maßnahme aufgrund öffentlich-rechtlicher Vorschriften von einem Sozialleistungsträger getragen oder auch nur bezuschusst worden sind ${ }^{122}$.

\section{Medizinische Rehabilitation in der gesetzlichen} Krankenversicherung

Im Vorangegangenen ist bereits gelegentlich das Recht der gesetzlichen KV angesprochen worden insoweit, als es auch dort Leistungen zur Rehabilitation gibt. Dabei wurde auch festgestellt, dass zwar nicht ausdrücklich, jedoch der Sache nach Rehabilitation traditionell auch in diesem Rechtsbereich angesiedelt ist ${ }^{123}$.

Es wurde ebenfalls bereits erwähnt, dass es sich bei der medizinischen Rehabilitation letztlich um Krankenversorgung handelt, also um Maßnahmen de Heilbehandlung, die aber in ihrem Schwerpunkt anders ausgerichtet sind als die Maßnahmen der so genannten Akutbehandlung.

\section{1 Änderungen in der systematischen Zuordnung}

Die gesetzessystematische Zuordnung der Rehabilitation zur Krankenbehandlung hat sich in letzter Zeit geändert. In der früheren, bis 31. 12.1999 geltenden Fassung des $\$ 11$ SGB V stellte dessen Abs 2 klar, dass „zu den Leistungen nach Abs 1" auch die medizinischen und ergänzenden Rehabilitationsleistungen gehören. Damit knüpfte der frühere Abs 2 an die $\$ \$ 10,12$ RehaAnglG sowie

$\left.{ }^{119}\right)$ Dazu Welti, aaO $\$ 17$ Rn 3

$\left.{ }^{120}\right)$ Str, wie hier: Welti, aaO $\$ 17 \mathrm{Rn} 24$

$\left.{ }^{121}\right) \$ 15$ Abs 3 SGB VI.

$\left.{ }^{122}\right) \$ 12 \mathrm{Abs} 2 \mathrm{~S} 1 \mathrm{SGB}$ VI.

${ }^{123}$ ) Vgl oben 2.1 die Rechtsprechung an, die bereits davor den Ausgleich einer Behinderung und des dadurch verursachten Funktionsausfalls als medizinische Leistung angesehen und der Krankenpflege im Sinn der gesetzlichen KV zugeordnet hatte ${ }^{124}$ ).

Das „GKV-Gesundheitsreformgesetz 2000“125) hat diese Zuordnung der Rehabilitationsleistungen zu $\$ 11$ Abs 1 SGB V aufgegeben; nach dem ab 1.1.2000 geltenden Verständnis sollen Rehabilitationsleistungen offenbar weder ein Unterfall der Krankenbehandlung noch der Vorsorge sein. Vielmehr soll es sich nunmehr um eine eigenständige Leistung handeln. Dies hat alles zu den bekannten systematischen Unklarheiten geführt.

Im Zuge dieser „Neuformierung“ wurde im Übrigen auch die früher in $\$ 40$ Abs 1 SGB V geregelte ambulante Rehabilitationskur den ambulanten Vorsorgeleistungen zugeordnet, weil sie auf die Verhütung der Verschlimmerung einer Krankheit abziele ${ }^{126}$ ). Diesem neuen Verständnis widerspricht es allerdings, das die Rehabilitationsleistungen weiterhin der Krankenbehandlung in $\$ 27$ Abs 1 S. $2 \mathrm{Nr} 6$ SGB V zugeordnet sind und dass die Ansprüche auf Rehabilitation im fünften Abschnitt des SGB V („Krankenbehandlung“) platziert sind ${ }^{127}$ ).

Dem früheren „systematischen Gesamtverständnis“ wird - und das wiederum in einer gewissen Abweichungen zu dem mittlerweile wohl geltenden Trennungsprinzip (Trennung zwischen Krankenversorgung/Heilbehandlung einerseits und Rehabilitation andererseits) - hingegen eher wieder Rechnung getragen durch die Bestimmungen zur Frührehabilitation ${ }^{128}$ ) sowie zur Anschlussrehabilitation $\left.^{129}\right)$.

\section{2 Überblick über die Regelungen im SGB V}

\subsubsection{Anspruchsgrundlagen zu den "Leistungen zur medizinischen}

\section{Rehabilitation"}

Das SGB V enthält in seinem Überblick über die „Leistungsarten“ selbstverständlich auch die „Behandlung einer Krankheit" ${ }^{\prime 130}$ ); dabei wird auf die $\$ 27$ bis 52 SGB V verwiesen - und dort findet man (auch) die "Leistungen zur medizinischen Rehabilitation".

Dabei fällt zunächst $\$ 40$ SGB V ins Auge, der allgemein von „Leistungen zur medizinischen Rehabilitation" spricht ${ }^{131}$ ); auch die nachfolgende Vorschrift ( $\$ 41$ SGB V - „medizinische Rehabilitation für Mütter und Väter“) spricht diesen Leistungskomplex deutlich an.

124) KassKomm-Höfler $\$ 11$ SGB V Rn $12 \mathrm{mwN}$

${ }^{125)}$ V 22. 12. 1999, BGBl I 2626

${ }^{126)}$ BT-DS 14/1977, 160.

127) KassKomm-Höfler $\$ 11$ SGB V Rn 13

$\left.{ }^{128}\right) \mathrm{Vgl} \$ 39$ Abs $1 \mathrm{~S} 3$ (aE) SGB V.

$\left.{ }^{129}\right)$ Vgl $\$ 40$ Abs 6 SB V sowie übrigens auch $\$ 32$ Abs 1 SGB VI.

$\left.{ }^{130}\right) \S 11$ Abs $1 \mathrm{Nr} 4 \mathrm{SGB}$.

131) Die frühere Überschrift des mit dem Gesundheitsreformgesetz v 20. 12. 1988, BGBI I 2477, mWv 1. 1. 1989 eingeführten $\$ 40$ SGB V(„Medizinische Rehabilitationsmaßnahmen“) wurde mit der Einführung des SGB IX (Art $5 \mathrm{Nr} 8, \mathrm{mWv} 1.7 .2001$ ) in die heutige Fassung („Leistungen zur medizinischen Rehabilitation“) überführt. 
An dieser Stelle sei aber auch bereits darauf hingewiesen, dass die Leistungen nach $\$ 40$ Abs 1 und 2 SGB V nur erbracht (werden), wenn nach den für andere Träger der SV geltenden Vorschriften solche Leistungen nicht erbracht werden können "132); eine Ausnahme gilt für die Leistungen nach $\$ 31$ SGB VI - dabei handelt es sich um die „sonstigen Leistungen“ die von dem Träger der Rentenversicherung exklusiv zu erbringen sind. Mit dieser Vorrang-/Nachrangregelung bringt der Gesetzgeber zum Ausdruck, das in diesen Fällen gleichsam „tatbestandsmäßig" sowohl die KV als auch andere Rehabilitationsträger, vor allem die Rentenversicherung, zuständig sind; andernfalls wäre eine solche Vorrang/Nachrangregelung nicht erforderlich gewesen.

\subsubsection{1 „Ergänzung" der ambulanten Krankenbehandlung}

Die Regelungen in $\$ 40$ Abs 1 und 2 SGB V ergänzen ausweislich des klaren gesetzgeberischen Willens nur die ambulante Krankenbehandlung, und zwar durch Leistungen der medizinischen Rehabilitation, die ihrerseits entweder ambulant ( $\$ 40$ Abs 1 SGB V) oder - erforderlichenfalls - durch „stationäre Rehabilitation mit Unterkunft und Verpflegung“ ( $\$ 40$ Abs 2 SGB V) erbracht werden.

\subsubsection{Frührehabilitation}

Daneben wird - gesetzestechnisch weniger auffällig platziert - die Frührehabilitation gewährt, als Teil der akutstationären Behandlung im Krankenhaus ${ }^{133}$ ).

\subsubsection{Anschlussrehabilitation}

Als eine weitere Kategorie von Rehabilitation wird man wohl auch die "Anschlussrehabilitation" zählen müssen, die in $\$ 40$ Abs 6 SGB V im Zusammenhang mit der Regelung von Zuzahlungen (also nicht als eine selbstständige Anspruchsgrundlage, die gesetzestechnisch als solche ausgewiesen ist) im Gesetz erscheint.

In ähnlicher Weise definiert im Übrigen auch das Rentenversicherungsrecht, und dort ebenfalls im Zusammenhang mit Zuzahlungen bei Leistungen zur medizinischen Rehabilitation, die „Anschlussrehabilitation" als eine stationäre Heilbehandlung, die unmittelbar an eine Krankenhausbehandlung anschließt (und medizinisch notwendig ist); ebenso wie in $\$ 40$ Abs 6 SGB $V$ wird auch in $\$ 32$ Abs 1 SGB VI die „Unmittelbarkeit" festgelegt in dem Sinne, dass die Maßnahme innerhalb von 14 Tagen beginnen muss.

\subsection{Medizinische Rehabilitation für Mütter und Väter}

In ähnlicher Weise könnte die „medizinische Rehabilitation für Mütter und Väter“ gem $\$ 41$ SGB V konzipiert sein. Ein diesbezüglicher Anspruch ${ }^{134}$ ) besteht

$\left.{ }^{132}\right) \$ 40$ Abs 4 SGB V.

$\left.{ }^{133}\right) \$ 39 \mathrm{Abs} 1 \mathrm{~S} 3$, letzter HS SGB V

${ }^{134)}$ KassKomm-Höfler $\$ 41$ SGB V Rn 5a mwN für Versicherte dann, wenn sie krank sind; diese Voraussetzung ist durch die Bezugnahme auf $\$ 27$ Abs 1 SGB V festgelegt.

Von der Vorsorgeleistungen für Mütter und Väter nach $\$ 24 S_{\text {SB }} V^{135}$ ) unterscheiden sich die Rehabilitationsleistungen des $\$ 41$ SGB V durch die besondere Zielsetzung der Rehabilitation gegenüber der Vorsorge ${ }^{136}$ ) Mit dem GKVGesundheitsreformgesetz 2000 ist auch die Terminologie geändert worden; an die Stelle der "Müttergenesungskur" ist die "medizinische Rehabilitation" und die Stelle der "Rehabilitationskur" sind die "Leistungen der Rehabilitation" getreten; eine inhaltliche Änderung der Zielsetzung war damit nicht verbunden. Vielmehr sollten lediglich Zweifel am medizinischen Nutzen der Maßnahmen abgewendet werden, die man bei der Bezeichnung „Kur“" befürchtete ${ }^{137}$ ).

Dass eine Behinderung oder die Gefahr einer Behinderung gegeben sein muss, fordert der Gesetzgeber in dieser Vorschrift ausdrücklich nicht.

Somit kann man wohl annehmen, dass in diesem Falle Leistungen der medizinischen Rehabilitation unabhängig davon zu gewähren sind, ob eine Behinderung und die Gefahr einer Behinderung vorliegen. Damit würde der Sache nach eine neue Kategorie von Defiziten geschaffen worden seien, die zu Leistungen der medizinischen Rehabilitation berechtigt, ohne dass die prägenden Merkmale des SGB IX gegeben sein müssen.

\subsection{Belastungserprobung und Arbeitstherapie}

Der Sache nach lassen sich auch die „Belastungserprobung“ sowie die „Arbeitstherapie“ als Maßnahmen verstehen, die ihrem Wesen nach zur Rehabilitation gehören, wobei allerdings der „medizinische Anteil“ im Einzelfall wohl gering sein kann.

Das SGB V gibt Versicherten einen diesbezüglichen Anspruch, der allerdings im Hinblick auf eine gleichartigen Anspruch gegenüber einem anderen Träger der SV nachrangig ist ${ }^{138}$ ).

\subsection{Ergänzende Leistungen zur Rehabilitation}

Nach den Regelungen des SGB V können „ergänzende Leistungen zur Rehabilitation" erbracht werden ${ }^{139}$ ).

Diese Leistungen beinhalten, vor allem auch infolge der Verweisung auf zahlreiche Regelungen des SGB IX, mehrere Kategorien, die auch gesetzestechnisch getrennt geregelt sind.

$\left.{ }^{135}\right) \mathrm{Zu}$ den tatbestandlichen Überschneidungen vgl im Einzelnen einerseits $\$ 24$ und andererseits $\$ 41$ SGB V.

$\left.{ }^{136}\right)$ KassKomm-Höfler $\$ 41$ SGB V Rn 3 mwN.

${ }^{137}$ ) KassKomm-Höfler $\$ 41$ SGB V Rn $2 \mathrm{mwN}$; der frühere nicht ungebräuchlichen Charakterisierung entsprechend dem Slogan „Morgens Fango, abends Tango" sollte nachhaltig entgegengetreten werden.

$\left.{ }^{138}\right) \S 42$ SGB V.

$\left.{ }^{139}\right) \$ 43$ SGB V. 


\subsubsection{Medizinische Rehabilitation}

Hinsichtlich der medizinischen Rehabilitation wird im Rahmen der „ergänzenden Leistungen zur Rehabilitation " ${ }^{140}$ ) in generalklauselartiger Weise ein Anspruch auf diesbezügliche Leistungen (zur medizinischen Rehabilitation) eingeräumt, bei dem inhaltlich ebenfalls auf die Regelungen des SGB IX verwiesen wird. Folgerichtig ist Voraussetzung für diese Rehabilitationsleistungen, dass eine Behinderung vorliegt und dass die Leistung der Krankenkasse erforderlich ist, „um das Ziel der Rehabilitation zu erreichen oder zu sichern"141).

Diese Maßnahmen können entweder durch die Krankenkasse selbst oder auch durch die Förderung von Leistungen anderer Träger durchgeführt wer$\operatorname{den}^{142}$ ).

Diese Leistungen zur medizinischen Rehabilitation werden nur im Zusam menhang mit einer Krankenbehandlung seitens der Krankenkasse gewährt. Im Hinblick auf den Zeitablauf legt das Gesetz fest, dass die Krankenkasse zuvor geleistet haben muss - dann handelt es sich der Sache nach um „Anschlussrehabilitation“. Ausweislich des Gesetzeswortlautes ist es aber auch möglich, dass diese Leistungen während einer Krankenbehandlung, also zeitgleich mit dieser gewährt werden - in diesem Falle könnte man wohl von "Frührehabilitation“ sprechen, wobei allerdings bereits eine „Behinderung“ vorliegen muss, um auf diese Anspruchsgrundlage zurückgreifen zu können.

Auch aus gesetzessystematische Betrachtungsweise ist bemerkenswert, dass diese Rehabilitationsleistungen unabhängig davon erbracht werden, ob die Krankenbehandlung, mit der diese Leistung im Zusammenhang stehen muss, ambulant oder stationär erbracht wird; insoweit besteht ersichtlicherweise ein Unterschied sowohl zu den Leistungen der medizinischen Frührehabilitation gem $\$ 39$ Abs 1 SGB V als auch zu den "normalen“ Leistungen zur medizinischen Rehabilitation gem $\$ 40$ SGB V.

Alle Leistungen auf Ansprüche zur medizinischen Rehabilitation werden im Ubrigen bereits in $\$ 11$ Abs 2 SGB V dahingehend präzisiert, als diese Leistungen beansprucht werden können, um eine Behinderung oder Pflegebedürftigkei abzuwenden, zu beseitigen, zu mindern, auszugleichen, ihre Verschlimmerung zu verhüten oder ihre Folgen zu mildern. Diese Regelung steht offensichtlich insoweit im Einklang mit denen Vorschriften des SGB IX, als die Rehabilitation ihrem Wesen nach darauf ausgerichtet ist, eine Behinderung zu beseitigen oder zu verhindern.

\subsubsection{Sozialmedizinische Nachsorgemaßnahmen}

Der Kategorie der Leistungen zur medizinischen Rehabilitation lassen sich auch die „sozialmedizinischen Nachsorgemaßnahmen ${ }^{143}$ ) für chronisch Kranke

$\left.{ }^{140}\right)$ Gem $\$ 43$ SGB V.

$\left.{ }^{141}\right) \S 43$ Abs 1 Nr 1 SGB V.

$\left.{ }^{142}\right) \$ 43 \mathrm{Abs} 1 \mathrm{Nr} 1 \mathrm{SGB}$.

${ }^{143}$ ) Zum Stichwort "Sozialmedizin“ vgl den „Abschlussbericht der Kommission zur Weiterentwicklung der Sozialmedizin in der gesetzlichen Rentenversicherung" herausgegeben vom Verband Deutscher Rentenversicherungsträger (2004). oder schwerstkranke Kinder, ... (zuordnen) ..., wenn die Nachsorge wegen der Art, Schwere und Dauer der Behandlung notwendig ist, um den stationären Aufenthalt zu verkürzen oder die anschließende ambulante ärztliche Behandlung zu sichern ${ }^{\text {"144 }}$ ).

Mit dieser Regelung wird zugleich auch bestätigt, dass Nachsorgemaßnahmen durchaus auch schon in Phasen stattfinden können und sollen, in denen noch Akutbehandlung angezeigt ist, und zwar in den Fällen, in denen diese Leistung den stationären Aufenthalt verkürzen soll.

\subsubsection{Weitere Leistungen}

Nach $\$ 43$ Abs 1 SGB V werden neben dem Krankengeld ${ }^{145}$ ) weitere Leistungen erbracht; dabei verweist das SGB V auf das Kapitel 6 des SGB IX, in dem „unterhaltssichernde und andere ergänzende Leistungen" geregelt sind. Die Verweisung in $\$ 43 \mathrm{Abs} 1 \mathrm{Nr} 1 \mathrm{SGB} V \mathrm{bezieht}$ zum einen Geldleistungen und geldwerte Leistungen in den Bereich der „ergänzenden Leistungen zur Rehabilitation" ein beispielsweise Beiträge und Beitragszuschüsse, Reisekosten ${ }^{146}$ ) sowie Betriebsoder Haushaltshilfe und Kinderbetreuungskosten ${ }^{147}$ ).

Daneben werden aber auch Leistungen erbracht, die sich unmittelbar mit dem defizitären Zustand des Versicherten befassen. In $\$ 43$ Abs $1 \mathrm{Nr} 2$ SGB V werden „wirksame und effiziente Patientenschulungsmaßnahmen für chronisch Kranke" genannt. Über die Verweisung auf $\$ 44$ Abs 1 SGB IX wird weiterhin der "ärztlich verordnete Rehabilitationssport ${ }^{\text {“148 }}$ ) und das „ärztlich verordnete Funktionstraining ${ }^{\text {(149) }}$ ) in den Leistungskatalog einbezogen.

\subsection{Gestaltungsfreiheit der Verwaltung}

(Ermessen / Beurteilungsspielraum)

Auf Leistungen zur medizinischen Rehabilitation besteht ein Anspruch "dem Grunde nach". Der Verwaltung ist kein so genanntes Entschließungsermes"sen ${ }^{150}$ ) eingeräumt; jedoch bestimmt die Krankenkasse „nach den medizinischen Erfordernissen des Einzelfalles Art, Dauer, Umfang, Beginn und Durchführung der Leistungen ... sowie die Rehabilitationseinrichtung nach pflichtgemäßem Ermessen". Damit der ist der Verwaltung ein Auswahlermessen eingeräumt.

Der Gesetzgeber hat weiterhin bei den Leistungen zur medizinischen Rehabilitation folgende Restriktionen ${ }^{151}$ ) vorgesehen: „ambulante Rehabilitationsleistungen sollen für längstens 20 Behandlungstage, stationäre Rehabilitation soll

${ }^{144)} \S 43$ Abs 2 S 1 SGB V

${ }^{145)} \mathrm{S} \$ 44 \mathrm{ff}$ SGB V.

${ }^{146)}$ Näher geregelt in $\$ 53$ SGB IX.

$\left.{ }^{147}\right)$ Näher geregelt in $\$ 54$ SGB IX.

$\left.{ }^{148}\right) \$ 44$ Abs 1 Nr 3 SGB IX.

$\left.{ }^{149}\right) \$ 44 \mathrm{Abs} 1 \mathrm{Nr} 4 \mathrm{SGB}$ IX

${ }^{150}$ ) Vgl oben 4.8.

151) Man könnte auch von Rationierung sprechen; vgl auch Seewald, Rationierung/ Rationalisierung - die Rechtsfragen, in ders/Schoefer (Hrsg), Zum Wert unserer Gesundheit (2008) $29-58$. 
für längstens drei Wochen erbracht werden - es sei denn, eine Verlängerung der Leistung ist aus medizinischen Gründen erforderlich" ${ }^{152}$ ).

Diese Bindung gilt jedoch dann nicht, wenn der Spitzenverband und der Krankenkasse „, in Leitlinien Indikationen festgelegt und diesen jeweils eine Regeldauer zugeordnet hat; von dieser Regeldauer kann nur abgewichen werden, wenn dies aus dringenden medizinischen Gründen im Einzelfall erforderlich ist $^{\text {“153}) \text {. }}$

Weiterhin hat der Gesetzgeber festgelegt, dass Leistungen zur medizinischen Rehabilitation nicht vor Ablauf von vier Jahren nach Durchführung solcher oder ähnlicher Leistungen erbracht werden, deren Kosten aufgrund öffentlichrechtlicher Vorschriften getragen oder bezuschusst worden sind, es sei denn, ein vorzeitige Leistung ist aus medizinischen Gründen dringend erforderlich" ${ }^{\text {“154 }}$ ).

Schließlich ist gesetzlich festgelegt, dass medizinisch notwendige Leistungen zur Rehabilitation für versicherte Kinder, die das 14. Lebensjahr noch nicht vollendet haben, in der Regel für vier bis sechs Wochen erbracht werden ${ }^{155}$ ).

$\mathrm{Ob}$ die Verwaltung und ihre „Verwaltungshelfer" darüber hinaus eine Gestaltungsfreiheit haben bei der Frage, ob und in welchem Umfang Leistungen zur medizinischen Rehabilitation erbracht werden, hängt des weiteren davon $a b, o b$ bei der Umsetzung der im Gesetz verwendeten unbestimmten Rechtsbegriffe ein gerichtlich nicht überprüfbar Beurteilungsspielraum besteht.

\subsection{Verhältnis zu anderen Bereichen}

\section{(Rentenversicherung, Unfallversicherung)}

5.7.1 Medizinische Rehabilitation neben der ambulanten

\section{Krankenbehandlung}

Die Leistungen der gesetzlichen KV sind, soweit es die medizinische Rehabilitation gem $\$ 40$ SGB V betrifft, nachrangig gegenüber derartigen Leistungen anderer Träger der SV. Das bedeutet also, dass diese Leistungen von den Trägern der Rentenversicherung sowie der UV zwar vorrangig erbracht werden müssen, das aber durchaus auch die Zuständigkeit der KV gegeben sein kann.

$\mathrm{Zu}$ beachten ist allerdings, dass es hierbei nur um Leistungen zur Rehabilitation geht, die im Zusammenhang mit der ambulanten Krankenversorgung angezeigt sind.

Für die Rangfolge zwischen Leistungen zur medizinischen Rehabilitation, die nicht unter dieser Voraussetzung stehen, die also im Zusammenhang mit stationärer Krankenversorgung zu gewähren sind (zu denken ist hierbei die sowoh an die Frührehabilitation als auch an die Anschlussrehabilitation), gilt diese Abgrenzung nicht.

Im Übrigen wird die gesamte Problematik nicht dadurch einfacher, dass die Grenzen zwischen stationärer und ambulanter Behandlung nicht mehr in gleicher Weise, mit gleicher Sicherheit so gezogen werden können (jedenfalls aus

${ }^{152} \$ 40$ Abs 3 S 2 SGB V.

153) $\$ 40$ Abs 3 S 3 SGB V.

${ }^{154)} \$ 40$ Abs 2 S 4 SGB V

$\left.{ }^{155}\right) \$ 40$ Abs $3 \mathrm{~S} 5 \mathrm{iVm} \$ 23$ Abs 7 SGB V. rechtlicher Betrachtungsweise nicht), wie das noch vor einigen Jahren möglich war, in einer Zeit, als zumindest aus rechtlicher Sicht, wohl auch aus medizinisch-fachlicher Sicht, Abgrenzung mit größerer Sicherheit vorgenommen worden ist.

Fraglich ist im Hinblick auf $\$ 40$ Abs 4 SGB V in diesem Zusammenhang, in welchem Fall Leistungen zur medizinischen Rehabilitation von einem anderen SV-Träger "nicht erbracht werden können“. Allgemein wird hierzu bemerkt dass mit dieser Regelung den Versicherungsträgern Leistungen ermöglicht werden in Fällen, in denen andere SV-Träger nicht helfend eingreifen können ${ }^{156}$ ). Diese Voraussetzung ist dann gegeben, wenn zwar aus medizinischer Sicht Leistungen zur medizinischen Rehabilitation angezeigt sind, jedoch zusätzliche versicherungsrechtlich erforderliche Voraussetzungen nicht vorliegen. In der Rentenversicherung kann es sein, dass persönliche und versicherungsrechtliche Voraussetzungen nicht gegeben $\operatorname{sind}^{157}$ ). In der UV ist generell Voraussetzung für Ansprüche, auch auf Leistungen zur medizinischen Rehabilitation, dass die körperliche, geistige oder seelische Beeinträchtigung auf einen Arbeitsunfall oder Berufskrankheit zurückzuführen ist ${ }^{158}$ ).

\subsubsection{Ausnahme: § 31 SGB VI}

Eine Ausnahme ist für die "sonstigen Leistungen“ gem $\$ 31$ SGB VI festgelegt. Diese Leistungen dienen der Eingliederung von Versicherten in das $\mathrm{Er}$ werbsleben oder der Sicherung der Erwerbstätigkeit für solche Personen, die eine besonders gesundheitsgefährdende, ihre Erwerbsfähigkeit ungünstig beeinflussende Beschäftigung ausüben; weiterhin geht es dabei um Nach- und Festigungskuren wegen Geschwulsterkrankungen oder auch um Bezieher einer Rente wegen Alters, wegen verminderter Erwerbsfähigkeit oder einer Waisenrente, „wenn hierdurch voraussichtlich eine erhebliche Gefährdung der Gesundheit beseitigt oder eine beeinträchtigte Gesundheit wesentlich gebessert oder wiederhergestell werden kann" ${ }^{\text {(159) }}$.

Für diese Leistungen ist und bleibt also ausschließlich die Rentenversicherung zuständig.

\subsubsection{Kein Nachrang aufgrund von $\$ 13$ SGB V}

$\$ 13$ Abs 2 SGB VI enthält einen generellen Ausschlusstatbestand zugunsten des Rentenversicherungsträgers; nach dieser in der Praxis besonders wichtigen Bestimmung können in der Rentenversicherung Leistungen im Sinne des $\$ 40$ Abs 4 SGB V in der Phase akuter Behandlungsbedürftigkeit nicht erbracht werden, mit Ausnahme der „interkurrenten“ Erkrankungen. Diese Regelung zeigt deutlich, dass die Akutbehandlung - im Verhältnis von KV zu Rentenversicherung - vorrangig der KV obliegt.

KassKomm-Höfler $\$ 40 \mathrm{Rn} 29 \mathrm{mwN}$

${ }^{157)} \$ 9$ Abs $2, \$ 10$ und $\$ 11$ SGB VI.

$\left.{ }^{158}\right) \$ 26$ Abs 2 Nr 1 SGB VII.

$\left.{ }^{159}\right) \$ 31$ Abs 1 SGB VI. 
Über Einzelfragen (Ende der Akutbehandlung, Zuordnung bestimmter Therapieformen, besonders bei psychisch Kranken) sollen gem $\$ 13$ Abs 4 SGB VI die Rentenversicherungsträger mit den Spitzenverbänden der Krankenkassen Näheres vereinbaren. Allerdings haben die Leistungsträger in der „Vereinbarung zur Leistungsabgrenzung nach $\$ 13$ Abs 4 SGB VI“ vom 31. 1. 1993 im Wesentlichen nicht diese Fragen geregelt, sondern nur Einzelheiten der interkurrenten Erkrankungen ${ }^{160}$ ).

\subsubsection{Nachrang bei stationärer Rehabilitation}

Gemäß $\$ 40$ Abs 4 SGB V besteht Nachrang bei stationärer Rehabilitation in einer Rehabilitationseinrichtung, die nach den Vorschriften gewährt werden kann, die für andere SV-Träger gelten. Dabei kommen vor allem Leistungen der Rentenversicherung ( $\$ 15$ SGB VI) und der UV ( $\$ 33$ SGB VII $\left.{ }^{161}\right)$ ) in Betracht.

Allerdings sind diese anderen Leistungsträger für die stationäre Versorgung nur dann vorrangig verpflichtet, wenn - zum Beispiel im Fall der Rentenversicherung - die persönlichen und versicherungsrechtlichen Voraussetzungen ${ }^{162}$ ) gegeben sind.

Ob der Rentenversicherungsträger sein Ermessen tatsächlich im Sinne einer Leistungsgewährung ausgeübt, soll grundsätzlich nicht ausschlaggebend sein. Lehnt jedoch ein SV-Träger die Leistung gegenüber einem Versicherten bestandskräftig ab mit der Begründung, die Leistungsvoraussetzungen sei nicht erfüllt, so kann die Krankenkasse den Versicherten nicht an diesen SV-Träger verweisen; es besteht zudem kein Erstattungsanspruch gegenüber dem anderen SV-Träger ${ }^{163}$ )

\subsubsection{Leistungen an pflegebedürftige Versicherte}

Hinsichtlich der Beziehung zur Pflegeversicherung enthält $\$ 40$ Abs 3 Satz 6 und 7 SGB V eine Regelung, die der Sache nach einen Schadensausgleich darstellt. Danach zahlt die Krankenkasse der Pflegekasse einen Betrag in Höhe von $3072,-€$ für pflegebedürftige Versicherte, für die innerhalb von sechs Monaten nach Antragstellung keine notwendigen Leistungen zur medizinischen Rehabilitation erbracht worden sind - in diesem Fall war offensichtlich die Krankenkasse zuständig, hat jedoch ihre Pflicht versäumt.

Damit soll erreicht werden, dass die Krankenkasse notwendige Rehabilitationsleistungen an pflegebedürftige Versicherte zeitnah erbringt ${ }^{164}$ ).

Diese Regelung steht in engem systematischen Zusammenhang sowohl mit $\S 5$ Abs 2 SGB XI als auch mit der - ausnahmsweise - bestehenden Zuständigkeit

\footnotetext{
${ }^{160)}$ KassKomm-Höfler $\$ 40 \mathrm{Rn} 30 \mathrm{~b} \mathrm{mwN}$.

${ }^{161)}$ Vgl dazu auch \$11 Abs 5 SGB V

$\left.{ }^{162}\right) \$ \$ 9$ ff SGB VI.

${ }^{163)}$ So das BSG, Nachweise bei KassKomm-Höfler $\$ 40$ SGB V Rn $31 \mathrm{mwN}$

${ }^{164)}$ Der Gesetzgeber hat weiterhin für diese Fallgestaltung ein Entfallen diese Ausgleichs vorgesehen, wenn "die Krankenkasse die fehlende Leistungserbringung nicht zu vertreten hat"; damit wird in diese Erstattungsregelung ein Verschuldens- beziehungsweise Entschuldigungselement für eine bestimmte Sachverhaltskonstellation eingebaut.
}

der Träger der Pflegeversicherung für lediglich vorläufige Leistungen der medizinischen Rehabilitation unter den Voraussetzungen des $\$ 32$ SGB XI ${ }^{165}$ ).

\section{Medizinische Rehabilitation in der gesetzlichen Unfallversicherung \\ 6.1 Ganzheitliche "Heilbehandlung"}

Wie bereits erwähnt, umfasst die „Heilbehandlung“ in der gesetzlichen UV traditionell sämtliche Phasen der Krankenversorgung ${ }^{166}$ ). Eine Unterscheidung in Akutversorgung und daran anschließende Rehabilitation war in diesem Rechtsgebiet gesetzlich nicht vorgesehen und wurde auch in der Verwaltungspraxis so nicht getätigt. Vielmehr wurde die Versorgung der Versicherten, die aufgrund eines Arbeitsunfalls oder einer Berufskrankheit behandelt werden müssen, stets "ganzheitlich" verstanden.

Somit wurde (und wird auch heute noch) bereits während der stationären Akutversorgung mit dem Versicherten sowie auch mit den behandelnden Medizinern beispielsweise die Frage erörtert, welche berufliche Möglichkeiten dem Unfallopfer noch offen stehen beziehungsweise welche Erwerbstätigkeiten den Verletzten aller Wahrscheinlichkeit nach verschlossenen sein werden. Und diesbezüglich werden Maßnahmen, soweit das möglich ist, sofort eingeleitet.

Dies war (und ist nach wie vor) die Aufgabe der "Berufshelfer“, die also bereits in diesem frühen Stadium der Heilbehandlung über „alle geeigneten Maßnahmen " zur bestmöglichen Wiederherstellung der Gesundheit und der Erwerbsfähigkeit nachdenken und auch mit dem Versicherten beraten.

Diese Zielsetzung der gesetzlichen UV im Hinblick auf die Versorgung von Unfallopfern oder Berufskranken besteht unverändert weiter, auch nach der geltenden Rechtslage. Danach haben Versicherte nunmehr zwar Anspruch auf "Heilbehandlung einschließlich Leistungen der medizinischen Rehabilitation $^{\text {(167) }}$. Mit der jetzigen, besonderen Betonung der Rehabilitation als Teil der Heilbehandlung hat sich in der Sache jedoch praktisch nichts geändert Mit diesem Begriff (Rehabilitation) wird in der UV nach wie vor die Gesamtheit der Maßnahmen umfasst, die - aus der heute eher üblichen, differenzierenden Sicht - als Akutversorgung und medizinische Rehabilitation bezeichnet werden.

\subsection{Das Leistungsniveau}

Die Integration der Maßnahmen, die in den anderen Bereichen des SVRechts als solche der Rehabilitation bezeichnet werden, in die Versorgung der Unfallopfer und Berufskranken, wird weiterhin durch die - ebenfalls traditionelle - Zielsetzung dieses Aufgabenbereichs der gesetzlichen UV im Hinblick auf das Leistungsniveau verdeutlicht. Gem $\$ 26$ Abs 2 SGB VII haben die UV-Träger „mit allen geeigneten Mitteln möglichst frühzeitig den durch den Versicherungs-

$\left.{ }^{165}\right)$ „Vorläufige Leistungen zur medizinischen Rehabilitation“.

${ }^{166)}$ Siehe oben bei 2.4 .

$\left.{ }^{167}\right) \S 26$ Abs 1 SGB VII. 
fall verursachten Gesundheitsschaden zu beseitigen oder zu bessern, seine Verschlimmerung zu verhüten und seine Folgen zu mildern“.

\subsection{Der Umfang der Heilbehandlung}

Der „Umfang der Heilbehandlung umfasst insb Erstversorgung, ärztliche Behandlung, zahnärztliche Behandlung einschließlich der Versorgung mit Zahnersatz, Versorgung mit Arznei-, Verband-, Heil- und Hilfsmitteln, häusliche Krankenpflege, Behandlung in Krankenhäusern und Rehabilitationseinrichtungen, Leistungen zur medizinischen Rehabilitation nach $\$ 26$ Abs $2 \mathrm{Nr} 1$ und 3 bis 7 und Abs 3 SGB IX“168).

\subsection{Die Verbindung zwischen SGB VII und SGB IX}

Die Verbindung zwischen dem Recht der gesetzlichen UV (SGB VII) und dem SGB IX wird in der Weise hergestellt, dass sich der Anspruch auf Heilbehandlung grundsätzlich nach den Vorschriften des SGB VII „und unter Beachtung des SGB IX“ richtet $^{169}$ ), auch hinsichtlich der Leistungen zur medizinischen Rehabilitation. Letzteres wird in $\$ 27$ Abs 1 Nr 7 SGB VII konkretisiert; danach umfasst die Heilbehandlung ,insb Leistungen zur medizinischen Rehabilitation nach $\$ 26$ Abs $2 \mathrm{Nr}$ lund 3 bis 7 und Abs 3 SGB IX“.

Somit richten sich die Maßnahmen, die nach dem heutigen rechtssystematischen Verständnis der medizinischen Rehabilitation zuzuordnen sind, vorrangig nach den Regelungen des SGB VII und ergänzend nach den Bestimmungen des SGB IX.

Ausdrücklich geregelt ist auch in diesem Rechtsbereich die stationäre Behandlung; dabei werden - konsequenterweise - Krankenhäuser und Rehabilitationseinrichtungen gleichsam in einem Atemzug genannt. Für beide Behandlungseinrichtungen und darauf bezogene Ansprüche der Versicherten gilt, dass diese Behandlung (in Krankenhäusern und Rehabilitationseinrichtungen) erbracht wird, „wenn die Aufnahme erforderlich ist, weil das Behandlungsziel anders nicht erreicht werden kann"170).

\subsection{Die Durchführung der Heilbehandlung}

Das im Bereich der gesetzlichen UV nach wie vor selbstverständliche $\mathrm{Ne}$ beneinander von Akutversorgung und rehabilitativen Maßnahmen wird auch deutlich in der Bestimmung über die "Durchführung der Heilbehandlung ${ }^{1711}$ ).

Danach haben die UV-Träger „alle Maßnahmen zu treffen, durch die eine möglichst frühzeitig nach dem Versicherungsfall einsetzende und sachgemäße Heilbehandlung und, soweit erforderlich, besondere unfallmedizinische oder

$\left.{ }^{168}\right) \$ 27$ Abs 1 SGB VII.

$\left.{ }^{169}\right) \S 26$ Abs 1 SGB VII.

$\left.{ }^{170}\right) \$ 33$ Abs 1 S 1 SGB VII.

$\left.{ }^{171}\right) \$ 34$ Abs 1 SGB VII.
Berufskrankheiten-Behandlung gewährleistet wird. Sie können zu diesem Zweck die von den Ärzten und Krankenhäusern zu erfüllenden Voraussetzungen im Hinblick auf die fachliche Befähigung, die sächliche und personelle Ausstattung sowie die zu übernehmenden Pflichten festlegen. Sie können daneben nach Art und Schwere des Gesundheitsschadens besondere Verfahren für die Heilbehandlung vorsehen ${ }^{\text {"172) }}$.

Hinsichtlich der Gestaltungsfreiheit der Träger der gesetzlichen UV im Einzelfall gilt auch in diesem Bereich, dass ein Ermessen im Hinblick auf Art, Umfang und Durchführung der Heilbehandlung - also lediglich ein so genanntes Auswahlermessen - besteht, das „pflichtgemäß $ß^{\text {“ }}$ zu betätigen ist ${ }^{173}$ ).

Dabei haben die UV-Träger auch zu prüfen, welche Leistungen geeignet und zumutbar sind, Pflegebedürftigkeit zu vermeiden, zu überwinden, zu mindern oder ihre Verschlimmerung zu verhüten ${ }^{174}$ ). Damit haben die Leistungen zur medizinischen Rehabilitation der UV Vorrang auch vor den Leistungen zur Pflege $^{175}$ ).

\subsection{Die Verfahrenstypen der unfallversicherungsrechtlichen} Heilbehandlung

Auf der Grundlage der Ermächtigungen in $\$ 34$ SGB VII hat die UV bestimmte Verfahren der Heilbehandlung festgelegt und entsprechende Vereinbarungen mit Ärzten und Krankenhäusern getroffen. Es wird unterschieden zwischen der "allgemeinen Heilbehandlung" im Rahmen der Verträge nach $\$ 34$ Abs 3 SGB VII und besonderen Verfahrensarten (gem $\$ 34$ Abs 1 S 3 SGB VII). ${ }^{176}$ )

Die Praxis der uv-rechtlichen Heilbehandlung kennt darüber hinaus drei definierte Verfahrenstypen: Beim „Durchgangsarzt-Verfahren“ (D-Arzt-Verfahren) werden Fachärzte mit bestimmter sächlicher und persönlicher Ausstattung unter Vertrag genommen, die nach einem Arbeitsunfall zuerst in Anspruch genommen werden sollen. Das „H-Arzt-Verfahren“" ${ }^{177}$ ) kennt besondere, an der Heilbehandlung beteiligten Ärzte, die auch ohne Vorstellung beim DurchgangsArzt besondere Heilbehandlung einleiten dürfen. Das „Verletztenartenverfahren" sieht vor, dass bei katalogmäßig bezeichneten Verletzungsarten in der Regel stationäre Behandlung in einem besonders zugelassenen Krankenhaus stattzufinden hat, gegebenenfalls nach Verlegung in eine solche Anstalt. Für Behandlungen von Nicht-Katalog-Verletzungen bedürfen Krankenhäuser keiner besonderen uv-rechtlichen "Zulassung"; hier kommt mit der Aufnahme allgemeine Heilbehandlung zu Lasten der UV-Träger zustande.

$\left.{ }^{172}\right) \S 34$ Abs 1 SGB VII.

$\left.{ }^{173}\right) \$ 26$ Abs 5 S 1 SGB V; siehe auch $\$ 39$ Abs 1 SGB I sowie oben 4.8 und 5.6.

$\left.{ }^{174}\right) \S 26$ Abs 5 S 2 SGB VII.

${ }^{175)}$ So Reimann, aaO Rn 206, mit Hinweis auf $\$ 44$ SGB VII; diese Vorschrift normiert einen uv-rechtlich-spezifischen Anspruch auf „Leistungen bei Pflegebedürftigkeit“" ${ }^{176}$ ) Vgl zum folgenden KassKomm-Ricke, \$34 SGB VII Rn 4- 10.

$\left.{ }^{177}\right){ }_{\text {}} \mathrm{H}^{\text {“ }}$ steht hier für (berufsgenossenschaftliches) Heilverfahren 


\subsection{Keine rationierende Leistungsbegrenzung}

Bemerkenswert ist für das Recht der gesetzlichen UV vor allem auch, dass in diesem Rechtsbereich keine weiteren gesetzlichen Vorgaben, insb auch nich hinsichtlich einer zeitlichen Begrenzung des Aufenthalts von Rehabilitanden in einer stationären Rehabilitationseinrichtung, existieren. Das bedeutet, dass die stationäre rehabilitative Maßnahme, unter Umständen bereits „parallel“ zu einer medizinischen Akutversorgung, solange fortgesetzt wird, bis das Behandlungsziel erreicht worden ist ${ }^{178}$ ).

\subsection{Verletztengeld bei medizinischer Rehabilitation}

Erwähnenswert ist weiterhin, vor allem auch unter dem Gesichtspunkt des integrativen Verständnisses von Akutbehandlung und Maßnahmen der Rehabilitation, zusammengefasst in der „Heilbehandlung“, dass das so genannte „Verletztengeld" der gesetzlichen UV (diese Lohnersatzleistung entspricht dem Krankengeld in der gesetzlichen KV) erbracht wird, wenn Versicherte „infolge des Versicherungsfalls arbeitsunfähig sind oder wegen einer Maßnahme der Heilbehandlung eine ganztägige Erwerbstätigkeit nicht ausführen können “ ${ }^{179}$ ).

Daraus wird zutreffend gefolgert, dass das Verletztengeld auch während der Leistungen zur medizinischen Rehabilitation gezahlt wird ${ }^{180}$ ).

\subsection{Vorrang / Nachrang der Leistungspflicht}

Die Abgrenzung der Zuständigkeit zwischen gesetzlicher UV und der KV sowie der Rentenversicherung ist insoweit vergleichsweise trennscharf, als Leistungen, die als Folge eines Arbeitsunfalls oder anders̀wo eine Berufskrankheit im Sinne der gesetzlichen UV zu erbringenden, von der KV nicht erbracht werden $^{181}$ ). Eine entsprechende Regelung enthält das Rentenversicherungsrecht; dort werden „Leistung zur Teilhabe" ebenfalls nicht für Versicherte erbracht, die wegen eines Arbeitsunfalls oder einer Berufskrankheit gleichartige Leistungen eines anderen Rehabilitationsträgers - und das sind auch die Träger der gesetzlichen UV - erhalten können ${ }^{182}$ ). Somit ist die gesetzliche UV gewissermaßen „exklusiv“ für die Rehabilitation „ihrer" Verletzten zuständig.

\subsection{Ergänzende Leistungen}

$\mathrm{Zu}$ den (ergänzenden) Leistungen zählen im Bereich der gesetzlichen UV infolge der Verweisung auf die einschlägigen Vorschriften des SGB IX - auch de

${ }^{178)}$ Zum Ermessen der UV-Träger hinsichtlich Art, Umfang und Durchführung der Heilbehandlung im Einzelfall („Auswahlermessen“, vgl dazu oben 6.5 sowie 4.8 und 5.6) $s \$ 2$ Abs5 SGB VII

$\left.{ }^{179}\right) \S 45$ Abs 1 Nr 1 SGB VII

$\left.{ }^{180}\right)$ Reimann, aaO Rn 212

$\left.{ }^{181}\right) \$ 11$ Abs 5 SGB V

$\left.{ }^{182}\right) \S 12$ Abs 1 Nr 1 SGB VI ärztlich verordnete Rehabilitationssport ${ }^{183}$ ) und das ärztlich verordnete Funktionstraining ${ }^{184}$ ).

Eine gegenüber dem SGB IX eigenständige ergänzende Leistung enthält das UV-Recht insoweit, als Kraftfahrzeughilfe gewährt wird ${ }^{185}$ ); weiterhin sind die ,sonstigen Leistungen zur Erreichung und zur Sicherstellung des Erfolges der Leistungen zur medizinischen Rehabilitation und Teilhabe ${ }^{\text {"186) }}$ zu nennen. Beide Leistungskomplexe sollen es dem Versicherten ermöglichen, trotz Art und Schwere des ihm verbliebenen Gesundheitsschadens am Arbeitsleben und am Leben in der Gesellschaft teilzunehmen ${ }^{187}$ ).

\section{Medizinische Rehabilitation in der Pflegeversicherung \\ 7.1 Keine Zuständigkeit zur Erbringung von Leistungen}

Die SGB XI (Soziale Pflegeversicherung) enthält keine eigenständigen Ansprüche auf Leistungen zur medizinischen Rehabilitation. Demnach sind die Träger der Pflegeversicherung keine potentiell zuständigen Rehabilitationsträger und werden als solche im SGB IX nicht genannt ${ }^{188}$ ).

Damit wird von den Zielen das Rehabilitationsrecht insoweit abgewichen als Leistungen der medizinischen Rehabilitation grundsätzlich auch erbracht werden, um unter anderem Pflegebedürftigkeit zu vermeiden, zu überwinden, $\mathrm{zu}$ mindern, eine Verschlimmerung zu verhüten ${ }^{189}$ ) beziehungsweise - in der Sprache des SGB V - Pflegebedürftigkeit abzuwenden oder ihre Folgen zu mindern ${ }^{190}$ ). Bei der gedanklich möglichen Erweiterung der Aufgaben der Pflegeversicherung im Hinblick auf die medizinische Rehabilitation wäre an die sicherlich nicht seltenen Pflegefälle zu denken, bei denen zugleich eine Behinderung vorliegt oder die Gefahr einer Behinderung besteht beziehungsweise die Leistungen der medizinischen Rehabilitation nach deren eigenen Zielsetzungen angezeigt sind.

Diesen Zusammenhang hat der Gesetzgeber offensichtlich auch gesehen und dadurch berücksichtigt, dass Leistungen zur medizinischen Rehabilitation, die eine ambulante Krankenbehandlung ergänzen sollen, auch in stationären Pflegeeinrichtungen zu erbringen sind; dabei handelt es sich um ambulante Rehabilitationsleistungen ${ }^{191}$ ).

\subsection{Ausnahme: Vorläufige Leistungen}

In Abweichung von der grundsätzlichen Unzuständigkeit erbringt die Pflegekasse „vorläufige Leistungen zur medizinischen Rehabilitation“. Vorausset-

$\left.{ }^{183}\right) \$ 44$ Abs 1 Nr 3 SGB IX

$\left.{ }^{184}\right) \$ 44$ Abs 1 Nr 4 SGB IX.

$\left.{ }^{185}\right) \S 39 \mathrm{Abs} 1 \mathrm{Nr} 1$ und $\$ 40$ SGB VII.

${ }^{186)} \$ 39$ Abs $1 \mathrm{Nr} 2$ SGB VII.

187) Reimann, aaO Rn, 217, 218, dort mwN in Fn 62.

$\left.{ }^{188}\right) \$ 6$ SGB IX.

$\left.{ }^{189}\right) \$ 26$ Abs 1 Nr 2 SGB IX.

$\left.{ }^{190}\right) \$ 11$ Abs 2 SGB V

191) VgI $\$ 40$ Abs 1 S 2 SGB V. 
zung hierfür ist, dass eine unmittelbar drohende Pflegebedürftigkeit vermieden werden soll; alternativ besteht diese Verpflichtung auch dann, wenn eine bestehende Pflegebedürftigkeit zu überwinden, zu mindern oder eine Verschlimmerung der Pflegebedürftigkeit zu verhüten ist. Gemeinsame Voraussetzung für diese Leistungen ist, dass ohne vorläufiges Tätigwerden die sofortige Einleitung der Leistungen gefährdet wäre ${ }^{192}$ ).

Hinsichtlich des Verfahrensablaufes ist vom Gesetzgeber vorgeschrieben, dass die Pflegekasse vor den eigenen Maßnahmen den zuständigen Träger unterrichten und dabei auf die Eilbedürftigkeit der Leistungsgewährung hinweisen muss; wird der zuständige Träger nicht rechtzeitig, spätestens jedoch vier Wochen nach Antragstellung, tätig, wird die Pflegekasse zuständig $\left.{ }^{13}\right)$.

Der Gesetzgeber hat im Hinblick auf die Krankenkassen diese Verpflichtung dadurch verstärkt, dass in den Fällen, in denen notwendige Rehabilitationsleistungen an pflegebedürftige Versicherte nicht zeitnah erbracht worden sind, eine Ausgleichszahlung an die Pflegekasse stattfindet $\left.{ }^{194}\right)$.

\subsection{Pflichten der Rehabilitationsträger im Hinblick} auf Pflegebedürftigkeit

In den Fällen, in denen Pflegebedürftigkeit bereits vorliegt, haben alle Leistungsträger im Rahmen ihres Leistungsrechts auch Maßnahmen zur medizinischen Rehabilitation (so wie im Übrigen auch ergänzende Leistungen) „in vollem Umfang" einzusetzen und darauf hinzuwirken, dass die Pflegebedürftigkeit überwunden, gemindert oder eine Verschlimmerung verhindert wird ${ }^{195}$ ).

Daneben gilt die allgemeine Zielsetzung bei Leistungen zur medizinischen Rehabilitation, der zufolge bei der Erbringung dieser Leistungen unter anderem auch Pflegebedürftigkeit überwunden, gemindert, eine Verschlimmerung verhütet und - darüber hinaus - sogar vermieden werden soll ${ }^{196}$ ).

Bestätigt wird dies durch eine weitere Regelung im SGB IX, wonach der Grundsatz des „Vorganges von Leistungen zur Teilhabe“ (also einschließlich der Leistungen zur medizinischen Rehabilitation) auch insoweit gilt, als durch diese Leistungen Pflegebedürftigkeit vermieden, überwunden, gemindert oder ihre Verschlimmerung verhütet werden soll. ${ }^{197}$ )

In die gleiche Richtung zeigt die allgemeine Regelung des SGB V zu den medizinischen Vorsorgeleistungen ", die unter anderem dann beansprucht werden können, wenn Pflegebedürftigkeit zu vermeiden ist ${ }^{198}$ ).

\footnotetext{
$\left.{ }^{192}\right) \S 32$ Abs 1 SGB XI.

$\left.{ }^{193}\right) \$ 32 \mathrm{Abs}, 2 \mathrm{SGB} X \mathrm{XI}$

${ }^{194)}$ Vgl oben 5.5.5 sowie $\$ 40$ Abs 3 S 6, 7 SGB V.

195) $\$ 5$ Abs 2 SGB XI.

$\left.{ }^{196}\right) \$ 26$ Abs 1 Nr 2 SGB IX.

${ }^{197)} \$ 8$ Abs 3 SGB IX.

$\left.{ }^{198}\right) \S 23 \mathrm{Abs} 1 \mathrm{Nr} 4 \mathrm{SGB} \mathrm{V}$
}

Überall dort, wo den Leistungsträgern Ermessen eingeräumt ist bei der Entscheidung über Art und Weise der medizinischen Rehabilitation ${ }^{199}$ ), gelten diese gesetzlichen Vorgaben (auch) als Ermessensrichtlinien ${ }^{200}$ ).

\section{Organisationsrechtliche Regelungen. Probleme und Entwicklungen}

Die bisherigen Ausführungen galten im Wesentlichen der materiell-rechtlichen Rechtslage im Hinblick auf Leistungen der medizinischen Rehabilitation. Dabei hat sich gezeigt ${ }^{201}$ ), dass zwar - einerseits - eine deutliche Vereinheitlichung der Begrifflichkeiten der diesbezüglichen rechtlichen Regelungen angestrebt wird zum Teil auch stattgefunden hat ${ }^{202}$ ), dass jedoch auch - andererseits - spezifische Eigenarten der Besonderen Teile zu unterschiedlichen Leistungsvoraussetzungen führen.

Weiterhin ist deutlich geworden, dass Rehabilitation ein Teil der Krankenversorgung ist; die Aufteilung dieses Geschehens in verschiedene Phasen - Akutversorgung, nachfolgende Rehabilitation, möglicherweise auch noch eine „Zwischenphase ${ }^{\text {(203 }}$ ) - suggeriert die Sinnhaftigkeit von entsprechend zeitlich aufeinander folgenden medizinischen Interventionen. Diese Vorstellung ist sicherlich traditionell bedingt; sie kann ihre Berechtigung jedoch auch aus einer entsprechenden medizinisch-professionellen Sachkunde ziehen, an die rechtliche Regelungen an sich durchaus anknüpfen sollten. Aus medizinischer Sicht bieten sich auch nach wie vor zahlreiche Ausprägungen dieser konsekutiv gestalteten Abfolge an. Einige Erkenntnisse im Medizinbereich und - diese berücksichtigend - auch im Bereich der Gesundheitspolitik sowie darauf beruhende Änderungen der Gesetzeslage haben bisherige Probleme des Verwaltungsvollzugs verschärf und neue Probleme geschaffen. Der Gesetzgeber hat diese Probleme erkannt; seine Lösungen sind im Wesentlichen im SGB IX festgelegt worden.

\section{Organisatorische Lösungen der Kollisionen im Bereich der medizinischen Rehabilitation}

\subsection{Aufbauorganisatorischer Ansatz}

Eine sich aufdrängende Lösung der Probleme liegt darin, die gesamte Krankenbehandlung - von der Diagnose, Akutversorgung, Früh-, Anschluss- und sonstiger medizinischer Rehabilitation - einem einzigen SV-Träger zuzuweisen - nahe liegend wäre die Zuständigkeit der gesetzlichen KV. Diesem Versiche-

199) Zum Auswahlermessen vgl 4.8, 5.6, 6.5, 6.7

$\left.{ }^{200}\right) \mathrm{ZB}$ in $\$ 23$ Abs 5 SGB V.

201) Insb auch unter Berücksichtigung der Entwicklung dieses Leistungskomplexes in der Vergangenheit.

${ }^{202}$ ) Zum Beispiel hinsichtlich der Einordnung der "medizinischen Rehabilitation“ in die "Leistungen zur Teilhabe" sowie hinsichtlich des gesetzestechnischen Versuches die Tatbestände der „Behinderung" sowie der "Gefahr einer Behinderung" als einheitliche Grundtatbestände in das gesamte Rehabilitationsrecht des SGB zu etablieren.

${ }^{203}$ ) Vgl oben bei 2.2 . 
rungszweig könnte die Heilbehandlung insgesamt zugewiesen werden, etwa nach dem ganzheitlichen Modell, das traditionell in der gesetzlichen UV verwirklicht wird.

Damit würde also die Rehabilitation aus der Rentenversicherung in die KV überführt werden; selbstverständlich schließt das nicht aus, dass Auswirkungen einer Krankheit auch unter dem Gesichtspunkt einer wie auch immer abgestuften Minderung/Beeinträchtigung/Einschränkung der Erwerbsfähigkeit ${ }^{204}$ ) medizinisch begutachtet und juristisch bewertet werden, mit entsprechenden rentenrechtlichen Rechtsfolgen.

Mit einer solchen Fortentwicklung des SV-Rechts würden allerdings auch Leistungsstandards der gesetzlichen UV beseitigt, die zu vergleichsweise günstigeren Leistungen sowohl in der Heilbehandlung als auch bei den Renten wegen einer Herabsetzung der Erwerbsfähigkeit ${ }^{205}$ ) berechtigen. Eine Rechtfertigung dieser Unterschiede ist möglich, vor allem angesichts der weitgehenden „Ablösung " der Unternehmer-Haftpflicht ${ }^{206}$ ); sie ist freilich auch nicht selbstverständlich.

\subsection{Die geltende Rechtslage}

Der Gesetzgeber hat die Vielfalt des Rehabilitationsrechts, die daraus erwachsenen Ungleichheiten und die Probleme des Verwaltungsvollzuges nich zum Anlass genommen, diesen Rechtsbereich einem einzigen Träger zuzuordnen. Deshalb musste nach anderen Möglichkeiten gesucht werden, den letztlich maßgeblichen Interessen des Bürgers nach einer optimal-effektiven Hilfe trotz der teilweise unterschiedlichen Ausgestaltung der materiell-rechtlichen Rechtslage und vor allem trotz der Trägervielfalt gerecht zu werden. Das Ergebnis dieser Suche findet sich im SGB IX und zwar in organisationsrechtlichen Vorschriften die bereichsübergreifend und für alle Träger der Rehabilitationsträger bindend sind.

\subsubsection{Die einzelnen Problemfelder}

Die materiell-rechtliche Rechtslage dem Bereich der medizinischen Rehabilitation und die Vielfalt der Leistungsträger führt zwangsläufig zu typischen Situationen, die sich aus rechtlicher Sicht ${ }^{207}$ ) als problematisch erweisen. Diese Probleme sind keineswegs unbekannt. Der Gesetzgeber hat insb mit den Regelungen des SGB IX den Versuch gemacht, dass Leistungsgeschehen so zu koordinieren und die Zusammenarbeit der Rehabilitationsträger so zu gestalten, dass im Einzelfall sämtliche rechtsverbindlichen Zielsetzungen der Kranken- bzw

${ }^{204}$ ) Nach dem früher geltenden Modell von „Berufsunfähigkeit“ oder „Erwerbsunfähigkeit“ oder dem derzeitigen Modell, das zwischen „teilweiser" und „voller Erwerbsminderung" unterscheidet, vgl $\$ 43$ Abs 1, 2 SGB VI

${ }^{205}$ ) Vgl hierzu die Rentenhöhe einerseits nach den Regeln der gesetzlichen UV $\$ \$ 43$ $-45,63$ ff SGB VII und andererseits nach der gesetzlichen Rentenversicherung, $\$ 56-91$ SGB VI.

${ }^{206)}$ Vgl im Einzelnen $\$ \$ 104-109$ SGB VII.

${ }^{207}$ ) Und angesichts der rechtlichen Gebundenheit der Leistungserbringung wohl auch aus medizinisch-praktischer Sicht.
Heilbehandlung einschließlich der Rehabilitationsleistungen optimal verwirklicht werden; demnach soll gewährleistet sein, dass die Behandlung rasch einsetzt, vollständig erbracht wird, dass kurative, präventive und rehabilitative Ziele erfüllt werden, dass verschiedene Leistungskomplexe nahtlos ${ }^{208}$ ) nicht nur ineinander übergehen, sondern erforderlichenfalls auch gleichzeitig ${ }^{209}$ ) getätigt werden und dass Art und Umfang einer rechtlich gleich zu gewährenden Leistung in der Praxis nicht unterschiedlich ausgestaltet wird, je nachdem, welcher Träger diese Leistung ausführt beziehungsweise diesen Anspruch erfüllt; weiterhin ist das Wunsch- und Wahlrechts des Leistungsberechtigten ${ }^{210}$ ) zu verwirklichen; schließlich soll das Leistungsgeschehen "ganzheitlich" gestaltet sein dergestalt, dass aus der Sicht des Leistungsberechtigten die insgesamt erforderlichen und geschuldeten Leistungen als „wie aus einer Hand erbracht" erscheinen.

Zur Erreichung dieses Gesamtziels ordnet der Gesetzgeber im SGB IX die Koordinierung der Leistungen und die Kooperation der Leistungsträger sowoh unter materiell-rechtlichen Gesichtspunkten als auch unter verfahrensrechtlichen Aspekten an.

Bereits an dieser Stelle sei allerdings auch schon auf ein Problem hingewiesen, dass vom SGB IX möglicherweise nicht gesehen, jedenfalls nicht ausdrücklich erwähnt und gelöst wird: dieses Gesetz (das SGB IX) bezieht sich nur auf Rehabilitationsleistungen, die unter spezifischen Voraussetzungen, nämlich einer vorhandenen oder einer drohenden Behinderung einsetzen sollen und die sich in ihrem Wesen wohl von anderen Maßnahmen der Kranken- bzw Heilbehandlung unterscheiden (sollen).

Konsequenterweise beziehen sich dann aber auch die Vorgaben zur Koordinierung und Kooperation nur auf dieses - für sich betrachtet, durchaus komplexe - Leistungsgeschehen. Nicht betroffen von diesen Regelungen sind demnach die "sonstigen“ Leistungen, die der für die Rehabilitation zuständige Träger dem betroffenen Patienten gegenüber zu erbringen hat, die unter dem Titel "Akutversorgung" firmieren und die nach heutigem Verständnis und der derzeitigen Rechtslage durchaus zeitgleich mit der spezifisch rehabilitativen Versorgung stattfinden können; in diesem Versorgungssegment bleiben die Träger offenbar autonom, handeln also ohne auch diesbezügliche Koordinations- oder Kooperationsverpflichtungen.

\subsubsection{Verfahrensrechtliche Koordinierung zur Abstimmung des}

\section{Leistungsgeschehens}

Der Gesetzgeber hat die Vollzugsprobleme des Rehabilitationsrechts gesehen. Der Ansatz des SGB IX ist umfassend: Mit den Leistungen sämtlicher Leistungsgruppen $^{211}$ ), den entsprechenden speziellen Leistungserbringern ${ }^{212}$ ) und den

\footnotetext{
${ }^{208}$ ) Also ohne vermeidbare zeitliche Verzögerung.
}

${ }^{209}$ ) Zum Beispiel Rehabilitationsmaßnahmen neben der Akutversorgung, also zeitgleich mit ihr.

${ }^{210}$ ) Vgl $\$ 9$ SGB IX sowie - allgemeiner - $\$ 33$ S 2 SGB I und oben bei 5.4.8.2.

211) Vgl $\$ 5$ SGB IX

$\left.{ }^{212}\right) \mathrm{Vgl} \$ 6$ SGB IX. 
gleichsam dahinter stehenden Leistungsträgern soll ganzheitlich auf Personen mit Defiziten eingewirkt werden, selbstverständlich unter deren Mitwirkung $\left.{ }^{213}\right)$. Und dies alles soll, vor allem im Hinblick auf die medizinische Rehabilitation, möglichst zügig erfolgen. Selbst wenn man sich auf diese Leistungsgruppe beschränkt, ist eine Koordination und Kooperation unter allen denkbaren Gesichtspunkten notwendig, wobei die Verzahnung mit den medizinischen Leistungskomplexen, die nicht in der Rehabilitation zuzuordnen sind, regelungstechnisch nicht dem SGB IX unterfällt $\left.{ }^{214}\right)$, jedoch aus gleichsam dringenden medizinischen Gründen ebenfalls stets und ständig zu berücksichtigen ist. lungen.

Der Gesetzgeber verfolgt dieses Ziel mit ambitioniert ausgestalteten Rege-

\subsubsection{1 § 10 SGB IX - Koordinierung der Leistungen}

$\$ 10$ SGB IX („Koordinierung der Leistungen“) regelt tatbestandsmäßig zwei Fallgruppen; in der einen erfordert das Ziel der Rehabilitation Leistungen verschiedener Leistungsgruppen und somit in der Regel wohl auch verschiedene Leistungsträger ; in der anderen - zweiten - Fallgruppe ist nur eine Leistungsgruppe einschlägig, zum Beispiel die der medizinischen Rehabilitation ${ }^{215}$ ), jedoch Leistungen mehrerer Träger erforderlich. Nach $\$ 10 \mathrm{Abs} 1 \mathrm{~S} 1$ in Verbindung mit $\$ 14$ SGB IX ist es im Falle einer mehrfach gegebenen "parallelen" Zuständigkeit (und auch bei Unklarheit der Zuständigkeit) einem dieser Trägern als „leistendem Rehabilitationsträger“ zusätzlich zu der ihm aufgegebenen Pflicht zur Erbringung „seiner" Leistungen die Verantwortung dafür aufgegeben, „dass die beteiligten Rehabilitationsträger im Benehmen miteinander und in Abstimmung mit den Leistungsberechtigten die nach dem individuellen Bedarf voraussichtlich erforderlichen Leistungen funktionsbezogen feststellen und schriftlich so zusammenfassen, dass sie nahtlos ineinander greifen “216); das gilt auch für die Zeit der Durchführung der Rehabilitationsmaßnahmen einschließlich eines unter Umständen notwendig werdenden Anpassungsbedarfs ${ }^{217}$ ).

Diese zusätzliche Pflicht fällt demjenigen der Rehabilitationsträger zu, der sich im Verfahren der „Zuständigkeitserklärung“ gem $\$ 14$ SGB IX als der „leistende Rehabilitationsträger" erwiesen hat, wobei § 14 SGB IX im Übrigen eher den Anschein erweckt, dass eigentlich ein einziger Rehabilitationsträger für die gesamte Leistungserbringung zuständig ist, wobei diese Zuständigkeitsfrage uU häufig schwierig zu beantworten ist

Wenn man allerdings berücksichtigt, dass der verfahrenseinleitende Antrag gem $\$ 14$ Abs 1 S 1 SGB IX nicht von vornherein konkret-gegenständlich formuliert sein muss $\mathrm{s}^{218}$ ), sondern sich zunächst allgemein auf die Rehabilitation als Verfahren beziehen kann $^{219}$ ), dann muss das Leistungsbegehren nicht zu einer

${ }^{213)} \mathrm{Vgl} \$ \$ 60-67$ SGB I.

${ }^{214}$ ) Vgl oben bei 9.2 .1 .

${ }^{215)} \$ 5 \mathrm{Nr} 1, \$ \$ 26-32$ SGB IX.

$\left.{ }^{216}\right) \$ 10$ Abs 1 S 1 SGB IX

$\left.{ }^{217}\right) \S 10$ Abs 1 S 2 SGB

${ }^{218} 8$ AA Benz, SGb 2001, 611, 613; Haines in LPK - SGB IX Rn 6 zu \$ 14.

$\left.{ }^{219}\right)$ BSGE 66, 87; BSG SozR $32200 \$ 1236 \mathrm{Nr} 3$ sowie $\$ 1237 \mathrm{Nr} 4$. bestimmten Leistung und zu einem einzigen dafür zuständigen Träger führen ${ }^{220}$ ). Somit ist die in $\$ 10$ SGB IX vorausgesetzte Vorstellung, der zufolge regelmäßig mehrere Rehabilitationsträger zuständig sind, um den gesamten Bedarf abzudecken, letztlich eher zutreffend. Zweckmäßig ist für diese Fallgestaltung dann auch die Bestimmung eines Rehabilitationsträgers, etwa als „primus inter pares“, der die notwendige Koordination verantwortlich vornimmt.

Dass der konkrete Bedarf an Leistungen - und das gilt wohl auch dann, wenn es allein um Leistungen zur medizinischen Rehabilitation geht - unter Umständen nur durch mehrere Rehabilitationsträger gedeckt werden kann und der diesbezügliche Komplexanspruch im Einzelfall verschiedene (Teil-) Ansprüche umfassen kann, diese Erfahrung berücksichtigt der Gesetzgeber auch, und zwar in $\$ 14$ Abs 6 SGB IX. Danach umfasst die Verantwortung des „primus-inter-pares-Rehabilitationsträgers" nicht nur die Prüfung der eigenen Zuständigkeit und der eigenen Pflicht zur Erfüllung des Anspruchs auf Rehabilitationsleistungen; sondern dieser „leistende Rehabilitationsträger“ hat auch zu prüfen, ob weitere Leistungen (die also über den eigenen Zuständigkeitsbereich hinausgehen) notwendig sind und welcher Rehabilitationsträger hierfür zuständig ist ${ }^{221}$ ). Dabei hat der Gesetzgeber sicherlich angenommen, das der "leistende Rehabilitationsträger" eine derartige Prüfung sachlich und rechtlich einwandfrei durchführen kann; an den seiner Auffassung nach zuständigen Träger wird das Verfahren mitkamn; dem Leistungsberechtigten weitergeleitet ${ }^{222}$ ).

Unklar ist, ob der somit einbezogene Rehabilitationsträger an die Entscheidung des "leistenden Rehabilitationsträgers" gebunden ist ${ }^{223}$ ) und ob dem Gesetzgeber bewusst war, dass (und welche) Konstellationen einer mehrfache $\mathrm{Zu}$ ständigkeit gegeben sein können, bei deren Vorliegen eine - gleichsam partielle, ergänzende - Weiterleitung wohl ausscheiden müssen ${ }^{224}$ ).

\subsubsection{Bildung von regionalen Arbeitsgemeinschaften}

Für das institutionalisierte Koordinationsgeschehen macht der Gesetzgeber weitere Vorgaben in der Regelung zur „Zusammenarbeit der Rehabilitationsträger" in \$12 SGB IX. Dabei wird eine aufbauorganisatorische Entscheidung getroffen, nämlich die Bildung von regionalen Arbeitsgemeinschaften, die als ständige Einrichtungen mit kooperativem und konsultativem Charakter dem gemeinsamen Handeln der am Rehabilitationsgeschehen Beteiligten einen stabilen Rahmen geben sollen ${ }^{225}$ ).

${ }^{220}$ ) Dass es unter Umständen eine Mehrfachzuständigkeit bei Rehabilitationsleistungen gibt, darauf wurde bereits hingewiesen, siehe oben bei $2.1-2.4,3.4 .4,9.1$.

${ }^{221}$ ) Diese Vorschrift erinnert an $\$ 14$ Abs 2 SGB I; auch dort wird davon ausgegangen, dass ein Sozialleistungsträger durchaus kundig ist im Hinblick auf Sach- und Rechtsfragen im Zuständigkeitsbereich eines anderen Leistungsträgers.

${ }^{222}$ ) Vgl \$ 14 Abs 6 S 1, iVm Abs 1 S 2 SGB IX.

${ }^{223}$ ) Zur Rechtsnatur dieser Entscheidung vgl Welti, aaO $\$ 14 \mathrm{Rn} 28 \mathrm{mwN}$

${ }^{224}$ ) Zur Wirkung des $\$ 14$ Abs 4 SGB IX (Erstattungsregelung) als Anreiz, sich im Zweifel für eine Weiterleitung zu entscheiden, vgl Welti, aaO \$14 Rn 24

225) Welti, aaO \$12 Rn 12 mit Hinweis auf BT-DS 14/5074, 101. 


\subsubsection{3 § 14 SGB IX - Zuständigkeitsklärung}

Ein Kernstück des SGB IX ist dessen $\$ 14$; diese Vorschrift enthält konkrete Vorgaben für den Verfahrensablauf im Anschluss an einen Antrag auf Leistungen zur Teilhabe, also auch auf Leistungen zur medizinischen dieser Regelung soll dafür gesorgt werden, dass medizinisch erforderliche und rechtlich geschuldete Leistungen möglichst schnell erbracht wird, wobei gesetzestechnisch der dabei zu klärenden, also wohl letztlich rechtsverbindlich festzustellenden Zuständigkeit eine zentrale Bedeutung zukommt $\mathrm{t}^{226}$ ).

$\$ 14$ SGB IX strukturiert das Verfahren vom Antrag bis zu dieser Entscheidung relativ subtil, unter Berücksichtigung verschiedener denkbarer Teil- (oder Vor-)Entscheidungen nach den einzelnen Verfahrensschritten. Die damit er zeugten Bindungen sind vor allem auch mit zeitlichen Vorstellungen des Gesetzgebers verbunden; man darf vermuten, dass damit einschlägige, negative Erfahrungen der vergangenen Jahrzehnte ausgewertet worden sind.

9.2.2.4 Ausführung der Leistungen durch ein persönliches Budget (§ 17 Abs 2 SGB IX)

Die Verantwortung des „leistenden Rehabilitationsträgers“(227) kann der Leistungsberechtigte auf sich selbst überleiten, indem er die Leistungserbringung im Wege eines „persönlichen Budgets“ beantragt ${ }^{228}$ ); damit soll vor allem wohl bei komplexen und lang anhaltenden Bedarfslagen die Koordination und Abstimmung des Leistungsgeschehens vom Rehabilitationsberechtigten selbst - als „Experte in eigener Sache ${ }^{\text {(2229) }}$ - organisiert werden.

Die Entscheidung, ob in dieser Form geleistet werden kann, steht im Ermessen des Rehabilitationsträgers ${ }^{230}$ ). Welcher Träger dieser Entscheidung zu treffen hat und bei welchem Träger die Verantwortung für die Erfüllung der Ansprüche auf Rehabilitationsleistungen verbleibt ${ }^{231}$ ), ist unklar, zumal $\$ 17$ SGB IX selbst - und insoweit in Abweichung von den in $\$ \$ 10-12$ SGB IX etablierten Erkenntnissen - offenbar davon ausgeht, dass ein einziger Rehabilitationsträger zuständig ist.

Unklar ist weiterhin, ob dieses Gesamtbudget als Geldleistung oder auch in Form von Gutscheinen oder Sachleistungsanrechten bei Diensten und Einrichtungen gewährt werden kann ${ }^{232}$ ).

\subsubsection{5 $§ 15 \mathrm{SGB} I X-$ Erstattung selbst beschaffter Leistungen}

Verfahrensrechtliche Bedeutung hat auch $₫ 15$ SGB IX; darin wird - vergleichsweise ausführlich - der Fall von Nichterfüllung eines Anspruchs auf Reha-

226) Vgl BT-DS 14/5074, 102.

$\left.{ }^{227}\right) \mathrm{Vgl}$ in diesem Zusammenhang auch den gesetzlich festgelegten „Anspruch auf ein Versorgungsmanagement" bei der Inanspruchnahme von Leistungen der GKV gem
$\S 11 \mathrm{Abs} 4 \mathrm{SGB}$ V.

${ }^{228}$ ) 17 Abs $1 \mathrm{Nr} 4$, Abs 2, 3 SGB IX

229) Welti, aaO $\$ 17 \mathrm{Rn} 18$

$\left.{ }^{230}\right) \mathrm{Vgl} \$ 17$ Abs 1 S $1, \$ 9$ Abs 1 SGB IX; ebenso Welti, aaO $\$ 17$ Rn 24; aA Mrozynski, aaO Rn $32-35$ zu $\$ 17$

$\left.{ }^{231}\right) \mathrm{Vgl} \$ 17$ Abs 1 S. 2 SGB IX.

232) Dazu Welti, aaO $\$ 17$ Rn 17 bilitationsleistungen wegen der Unmöglichkeit rechtzeitiger Leistungserbringung und - eher beiläufig - eine weitere Fallgruppe geregelt, bei der die Nichterfüllung dadurch bewirkt wird, das eine Leistung zu Unrecht abgelehnt worden ist ${ }^{233}$ ).

Der Zeitpunkt des Eintritts der Nichterfüllung ist für den Rehabilitationsberechtigten in beiden Fallgruppen klar erkennbar: Der Rehabilitationsträger muss - in der ersten Fallgruppe - sein Unvermögen dem Leistungsberechtigten mitteilen ${ }^{234}$ ); geschieht dies nicht oder liegt für das Unvermögen des Rehabilitationsträgers ein zureichender Grund nicht vor ", kann der Leistungsberechtigte sich (nach Ablauf "einer von ihm gesetzten Frist) „die erforderliche Leistung selbst beschaffen “235).

In der zweiten Fallgruppe (unrechtmäßige Ablehnung der Leistung) erfährt der Rehabilitationsberechtigte die Ablehnung der beantragten Leistung mit Bekanntgabe dieser Entscheidung ${ }^{236}$ ).

In den Fällen der Selbstbeschaffung liegt das Verfahren weitgehend in der Hand des Leistungsberechtigten; dieser wird damit in eigener Verantwortung zuständig. Problematisch ist bei rechtspraktischer Betrachtungsweise der Fall der zu Unrecht abgelehnten Leistung; denn insoweit wird der Selbstbeschaffung eine Beurteilung seitens des Leistungsberechtigten zugrundeliegen, die sich im Nachhinein - nämlich im Rahmen des wohl stets zu führenden Rechtsstreits wegen der Erstattungspflicht des Rehabilitationsträgers - auch als unzutreffend erweisen kann. Zugleich muss diese faktisch mit dem Risiko der (Nicht-)Erstattung behafteten Entscheidung aus medizinischen Gründen rasch und aus juristischen Gründen regelmäßig vor der Geltendmachung des Erstattungsanspruches getroffen und „vollzogen" werden, der tatbestandsmäßig getätigte Aufwendungen voraussetzt; allenfalls ist, wohl nur in besonderen Fällen, an eine Feststellungsklage ${ }^{237}$ ) zu denken.

Das Recht zu Selbstbeschaffung von Leistungen und der Anspruch auf Erstattung der dafür getätigten Aufwendungen berührt nicht die materiell-rechtliche Anspruchsgrundlage zwischen dem Leistungsberechtigten und einem oder mehreren Rehabilitationsträgern, einschließlich der dabei geltenden allgemeinen anspruchsbestimmenden Grundsätze von Wirtschaftlichkeit und Sparsamkeit ${ }^{238}$ ).

\subsubsection{Materiell-rechtliche Koordinierung und Kooperation}

Die eigentlich wesentliche Aufgabe des Rehabilitationsrechts ist die Ermittlung und Festlegung der Leistungen, die bei bestimmten Defiziten von Rechts wegen zu gewähren und - in Erfüllung dieser Pflichten - tatsächlich zu erbringen sind. In den damit angesprochenen materiell-rechtlichen Fragen wirken medizinischer Sachverstand und juristische Bewertung notwendigerweise zusammen.

An dieser Stelle soll allerdings nicht die - letztlich wohl nur entscheidungstheoretisch zu beantwortende - Frage des Zusammenwirkens dieser Professionen (Mediziner und Juristen) erörtert werden, obwohl darin auch eine Kernfrage der gerechten Zuteilung der gesetzlich verbürgten Leistungen steckt.

\footnotetext{
${ }^{233)} \mathrm{Vgl} \$ 15$ Abs 1 S 4 SGB IX.

$\left.{ }^{234}\right) \S 15$ Abs 1 S 1 SGB IX.

235) \$15 Abs 1 S. 2 SGB IX

236) $\$ 37$ SGB X.

$\left.{ }^{237}\right)$ Gem $\$ 55$ SGG.

${ }^{238)} \$ 15$ Abs 1 S 3 SGB IX.
} 
Vielmehr soll im nachfolgenden das Problem der - rechtlich verursachten - Vielfalt von Rehabilitationsträgern mit unter Umständen spezifischen Aufgaben und rehabilitativen Zielsetzungen und der - zusätzlich zu der Organisationsproblematik - sich aus der Natur der Sache ergebenden Vielfalt an rehabilitativen Maßnahmen, und zwar auch bereits allein im Bereich der medizinischen Rehabilitation, behandelt werden.

\subsubsection{Gemeinsame Empfehlungen}

Eines der auch materiell-rechtlichen Probleme besteht zunächst in der Abgrenzung der Maßnahmen zur medizinischen Rehabilitation von den sonstigen aus medizinischen Gründen indizierten Maßnahmen. Die Ermittlungen und Entscheidungen in diesem Problemfeld haben nicht nur Bedeutung für Art und Umfang eines Anspruchs, und zwar sowohl im Hinblick auf Maßnahmen der Akutversorgung als auch auf Leistungen der Rehabilitation, sowie für die Festlegung des zuständigen Rehabilitationsträgers (unter Umständen auch die Feststellung der parallelen Zuständigkeit mehrerer Träger). Unter dem Gesichtspunkt der Trägervielfalt und vor allem der gleichsam parallelen Leistungspflichten dem Grunde nach ${ }^{239}$ ) ist es naheliegend und aus dem Gesichtspunkt der verfassungsrechtlichen Gleichheitsgebote (und Diskriminierungsverbote) ${ }^{240}$ ) eine Pflicht der Verwaltungen, dafür Sorge zu tragen, dass bei gleich gelagerten Sachverhalten (hier im Hinblick auf medizinisch behandelbare Defizite) auch gleiche Leistungen erbracht werden.

Diesem Ziel dienen die "Gemeinsamen Empfehlungen“, die gem $\$ 13$ SGB IX von den Rehabilitationsträgern zu vereinbaren sind und der Sicherung der Zusammenarbeit nach $\$ 12$ Abs 1 SGB IX dienen sollen; damit sind die früheren "Gesamtvereinbarungen " ${ }^{\text {"241) }}$ ) abgelöst worden. Ausweislich der eingehenden Regelung durch den Gesetzgeber handelt es sich dabei offensichtlich um eines der zentralen Anliegen des Rehabilitationsrechts.

Rechtlich handelt es sich bei diesen "Gemeinsamen Empfehlungen“ um Verwaltungsvereinbarungen, also Verträge, die unmittelbare Rechtswirkungen nur zwischen den vertragsschließenden Beteiligten entfalten, somit allerdings auch deren Verwaltungshandeln (auf vertraglicher Grundlage) binden.

Eine Wirkung auf die Ansprüche der Versicherten wird über Art 3 Abs 1 GG erzeugt, entsprechend einer allgemeinen verwaltungsrechtlichen Dogmatik ${ }^{242}$ ), sie ist dort zu beachten, wo der Verwaltung ein Erkenntnis- oder Gestaltungsspielraum bei unbestimmten Rechtbegriffen und vor allem bei der Einräumung von Ermessen gewährt wird.

Eine wichtige Rolle spielt bei der Erarbeitung der „Gemeinsamen Empfehlungen“ die "Bundesarbeitsgemeinschaft für Rehabilitation" (BAR); diese bildet den organisatorischen Rahmen für die notwendigen Vorbereitungsund Abstimmungsprozesse und hat Initiativ-, Steuerungs- und Berichtaufga-

$\left.{ }^{239}\right) \mathrm{Vgl}$ dazu oben $3.1-3.3$

240) Vgl Art 3 Abs 1 - 3 GG

241) gem $\$ 5$ Abs 5 S 2 RehaAnglG.

${ }^{242}$ ) Für alle Maurer, aaO $\$ 24 \mathrm{Rn} 20$ - 26a; sa BVerwGE 112, 63 sowie 115, 189 und BVerfGE 78, 214, $227 \mathrm{ff}$ - zur Bindung der Gerichte an Verwaltungsvorschriften. ben ${ }^{243}$ ); die Empfehlungen der BAR können zusätzlich durch die regional zuständigen Rehabilitationsträger konkretisiert werden ${ }^{244}$ ).

Im Übrigen hat die BAR die gleiche Funktion bei der Erstellung der Gemeinsamen Empfehlungen zur Qualitätssicherung ${ }^{245}$ ); und auch bei den Verträgen der Rehabilitationsträger mit den Leistungserbringern haben die Träger zusammenzuarbeiten und die Arbeit der BAR zu nutzen ${ }^{246}$ ).

\subsubsection{Qualitätssicherung}

Für das Ziel eines einheitlichen Leistungsniveaus im Bereich der Rehabilitationsleistungen, und zwar unabhängig von der Zuständigkeit und der Ausführung dieser Leistungen durch den einen oder anderen Träger, sind auch die Vorschriften zur Qualitätssicherung ${ }^{247}$ ) einschlägig. Denn auch diesbezüglich verpflichtet der Gesetzgeber die meisten Rehabilitationsträger ${ }^{248}$ ) zur Vereinbarung „Gemeinsamer Empfehlungen“, die von der BAR vorzubereiten sind.

\subsubsection{Verträge mit Leistungserbringern}

Schließlich verpflichtet der Gesetzgeber die Rehabilitationsträger auch im Hinblick auf den Abschluss von Verträgen über die Ausführung von Leistungen durch Rehabilitationsdienste und -einrichtungen ${ }^{249}$ ) dahingehend, dass diese Verträge nach einheitlichen Grundsätzen abgeschlossen werden ${ }^{250}$ ). Zur Sicherung dieses Ziels sind die Rehabilitationsträger ermächtigt, auch über den Inhalt dieser Versorgungsverträge "Gemeinsame Empfehlungen " ${ }^{251}$ ) zu vereinbaren.

Weiterhin gelten diese einheitlichen Grundsätze auch für den Betrieb der Rehabilitationsdienste und -einrichtungen, die von den Rehabilitationsträgern in eigener Regie geführt werden ${ }^{252}$ ).

Schließlich können die Rehabilitationsträger „Rahmenverträge“ abschlieBen; Vertragspartner sind hierbei die „Bundesarbeitsgemeinschaften der Reha-

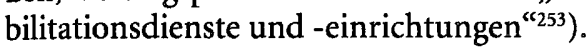

\subsection{4 „Aufbauorganisatorische Ergänzungen“ zur Kooperation der}

\section{Leistungsträger}

9.2.4.1 Gemeinsame Servicestellen

Als eine der organisatorischen Vorkehrungen zur Vermeidung von Unklarheiten infolge der komplizierten Rechts- und Organisationslage ist auch die Ein-

${ }^{243}$ ) Vgl $\$ 13$ Abs 5, 6 (Vorbereitung und Beteiligung von Interessenverbänden und Selbsthilfegruppen), Abs 7 (der „Rahmen“ der BAR), Abs 8 (Berichtspflicht) SGB IX sowie Welti, aaO $\$ 13 \mathrm{Rn} 13$ mit Hinweis auf BT-DS 14/5074,102.

${ }^{244)} \$ 13$ Abs 7 SGB IX.

${ }^{245}$ ) Siehe unten 11.2.3.2 sowie $\$ 20$ Abs 2, 2a SGB IX.

${ }^{246}$ ) $\$ 21$ Abs 2 SGB IX; Welti, aaO \$21 Rn 27.

$\left.{ }^{247}\right) \S 20$ SGB IX.

$\left.{ }^{248}\right) \mathrm{Vgl} \S 20$ Abs $1 \mathrm{~S} 1 \mathrm{iVm} \S 6$ Abs 1 Nr 1-5 SGB IX 6; die Träger der Jugendhilfe und der Sozialhilfe können diesen Empfehlungen beitreten, $\$ 20$ Abs 1 S 3 SGB IX.

${ }^{249}$ ) Dazu Näheres in \$19 SGB IX.

$\left.{ }^{250}\right) \$ 21$ Abs 2 S 1 erster HS SGB IX.

$\left.{ }^{251}\right) \mathrm{Gem} \$ 13$ SGB IX

${ }^{252)}$ Einen Überblick über die diesbezüglichen Regelungen gibt Welti, aaO $\$ 21 \mathrm{Rn} 7-18$

$\left.{ }^{253}\right) \S 21$ Abs 2 S 1, zweiter HS, SGB IX 
richtung der "gemeinsamen Servicestellen“ zu nennen, in denen „Beratung und Unterstützung " angeboten wird ${ }^{254}$ ). Die Aufgabenzuweisung an diese Stellen in $\$ 22$ SGB IX ergänzt und konkretisiert den allgemeinen Anspruch auf Beratung in $\$ 14$ SGB.

Weiterhin wird mit den „Servicestellen“ \$15 SGB I ergänzt und konkretisiert. Der Sache nach handelt es sich um organisatorische Festlegungen, die sich im Vorfeld des Leistungsgeschehens auswirken sollen insoweit, als die potentiel Berechtigten in die Lage versetzt werden sollen, sich einen zutreffenden Eindruck von den ihnen zustehenden materiell- und verfahrensrechtlichen Ansprüchen zu machen.

9.2.4.2 Die Bundesarbeitsgemeinschaft für Rehabilitation

Eine wesentliche Rolle hat die bereits erwähnte Bundesarbeitsgemeinschaft für Rehabilitation $\mathrm{eV}$ (BAR) im gesamten Rehabilitationsgeschehen zu erfüllen ${ }^{255}$ ). Der Gesetzgeber hat dieser Einrichtung, die sich zu Recht als gemeinsame Repräsentanz aller Rehabilitationsträger versteht ${ }^{256}$ ), an verschiedener Stelle eine wichtige Funktion bei der Koordination und Kooperation des Leistungsgeschehens übertragen.

Die "Gemeinsamen Empfehlungen" der Rehabilitationsträger beruhen auf einem Vorschlag der $\mathrm{BAR}^{257}$ ); mittelbar ist die BAR auch verantwortlich in den Fällen, in denen die Rehabilitationsträger nicht zeitgerecht Gemeinsame Empfehlungen vereinbaren ${ }^{258}$ ); auch die gemeinsamen Vereinbarungen im Hinblick auf die Qualitätssicherung werden von der BAR vorbereitet ${ }^{259}$ ); gleiches gilt für die „Verträge mit Leistungserbringern ${ }^{\text {“260)}}$. Weiterhin besteht eine Berichtspflich der Rehabilitationsträger beziehungsweise deren Spitzenverbände gegenüber der $\mathrm{BAR}^{261}$ ); schließlich bereitet die BAR die hier übermittelten Informationen auf im Sinne einer Berichts- und Erörterungspflicht ${ }^{262}$ ).

9.2.4.3 Weitere Bundesarbeitsgemeinschaften

Schließlich soll nicht unerwähnt bleiben, dass an der Vorbereitung der „Gemeinsamen Empfehlungen“ der Rehabilitationsträger auch weitere Stellen, unter anderem auch die „Bundesarbeitsgemeinschaft der überörtlichen Träger der Sozialhilfe" sowie die "Bundesarbeitsgemeinschaft der Landesjugendämter" beteiligt ist ${ }^{263}$ ).

${ }^{254)} \$ 22-25$ SGB IX.

255) Vgl oben 9.2.3.1, 9.2.3.2.

256) Die BAR informiert über Gemeinsame Empfehlungen, Rahmenempfehlungen, Arbeitshilfen, Reha-Infos, Arbeitsmaterialien und Verzeichnisse, Trägerübergreifendes persönliches Budget und Gemeinsame Servicestellen unter www.bar-frankfurt.de.

${ }^{257}$ ) 13 Abs 6 SGB IX.

258) Vgl $\$ 16$ SGB IX.

$\left.{ }^{259}\right) \$ 20$ Abs 2a, 3 SGB IX.

$\left.{ }^{260}\right) \S 21$ Abs 2 SGB IX

${ }^{261)} \$ 24$ Abs 1 SGB IX

$\left.{ }^{262}\right) \$ 24$ Abs 2 SGB IX.

${ }^{263)} \$ 13$ Abs 5 SGB IX).

\section{Anhang}

Medizinische Rehabilitation im Sozialversicherungsrecht - „Rehabilitationsrecht" -

\section{Auszüge aus dem Sozialgesetzbuch (SGB)} Sozialgesetzbuch (SGB) Erstes Buch (I) Allgemeiner Teil

\section{Teilhabe behinderter Menschen}

\$ 10. Menschen, die körperlich, geistig oder seelisch behindert sind oder denen eine solche Behinderung droht, haben unabhängig von der Ursache der Behinderung zur Förderung ihrer Selbstbestimmung und gleichberechtigten Teilhabe ein Recht auf Hilfe, die notwendig ist, um

1. die Behinderung abzuwenden, zu beseitigen, zu mindern, ihre Verschlimmerung zu verhüten oder ihre Folgen zu mildern,

2. Einschränkungen der Erwerbsfähigkeit oder Pflegebedürftigkeit zu vermeiden, zu überwinden, zu mindern oder eine Verschlimmerung zu verhüten sowie den vorzeitigen Bezug von Sozialleistungen zu vermeiden oder laufende Sozialleistungen zu mindern,

3. ihnen einen ihren Neigungen und Fähigkeiten entsprechenden Platz im Arbeitsleben zu sichern,

4. ihre Entwicklung zu fördern und ihre Teilhabe am Leben in der Gesellschaft und eine möglichst selbständige und selbstbestimmte Lebensführung zu ermöglichen oder zu erleichtern sowie

5. Benachteiligungen auf Grund der Behinderung entgegenzuwirken.

\section{Beratung}

5 14. Jeder hat Anspruch auf Beratung über seine Rechte und Pflichten nach diesem Gesetzbuch. ........

\section{Auskunft}

§ 15. (1) Die nach Landesrecht zuständigen Stellen, die Träger der gesetzlichen Krankenversicherung und der sozialen Pflegeversicherung sind verpflichtet, über alle sozialen Angelegenheiten nach diesem Gesetzbuch Auskünfte zu erteilen.

(2) Auskunftspflicht erstreckt sich auf .........

(3) ..... Auskunftsstellen..... möglichst umfassende Auskunftserteilung durch eine Stelle sicherzustellen.

(4)

\section{Ausführung der Sozialleistungen}

\$ 17. (1) Die Leistungsträger sind verpflichtet, darauf hinzuwirken, daß

1. jeder Berechtigte die ihm zustehenden Sozialleistungen in zeitgemäßer Weise, umfassend und zügig erhält, 
2.... erforderlichen sozialen Dienste und Einrichtungen rechtzeitig und ausreichend $\cdots$

3.... Zugang zu den Sozialleistungen möglichst einfach gestaltet ..

4.... den....

(2) ... Hörbehinderte Menschen haben das Recht, ... Gebärdensprache zu verwen-

(3) ... Zusammenarbeit mit gemeinnützigen und freien Einrichtungen und Organisationen ... Leistungsträger ...

\section{Leistungen der gesetzlichen Krankenversicherung}

5 21. (1) Nach dem Recht der gesetzlichen Krankenversicherung können in Anspruch genommen werden:

1. Leistungen zur Förderung der Gesundheit, zur Verhütung und zur Früherkennung von Krankheiten,

2. bei Krankheit Krankenbehandlung, insbesondere

a) ärztliche und zahnärztliche Behandlung,

b) Versorgung mit Arznei, Verband-, Heil- und Hilfsmitteln

c) häusliche Krankenpflege und Haushaltshilfe,

d) Krankenhausbehandlung,

e) medizinische und ergänzende Leistungen zur Rehabilitation,

f) Betriebshilfe für Landwirte,

g) Krankengeld,

3. bei Schwangerschaft und Mutterschaft ...,

4. Hilfe zur Familienplanung ...

5. (weggefallen)

(2) Zuständig sind die Orts-, Betriebs- und Innungskrankenkassen, ...

\section{Leistungen der gesetzlichen Unfallversicherung}

$\$$ 22. (1) Nach dem Recht der gesetzlichen Unfallversicherung können in Anspruch genommen werden:

1. Maßnahmen zur Verhütung von Arbeitsunfällen, Berufskrankheiten und arbeitsbedingten Gesundheitsgefahren und zur Ersten Hilfe sowie Maßnahmen zur Früherkennung von Berufskrankheiten und arbeitsbedingten Gesundheitsgefahren,

2. Heilbehandlung, Leistungen zur Teilhabe am Arbeitsleben und andere Leistungen zur Erhaltung, Besserung und Wiederherstellung der Erwerbsfähigkeit sowie zur Erleichterung der Verletzungsfolgen einschließlich wirtschaftlicher Hilfen,

3. Renten wegen Minderung der Erwerbsfähigkeit,

4. Renten an Hinterbliebene, Sterbegeld und Beihilfen,

5. Rentenabfindungen,

6. Haushaltshilfe,

7. Betriebshilfe für Landwirte.

(2) Zuständig sind die gewerblichen und die landwirtschaftlichen Berufsgenossenschaften, ...

\section{Leistungen der gesetzlichen Rentenversicherung einschließlich} der Alterssicherung der Landwirte

§ 23. (1) Nach dem Recht der gesetzlichen Rentenversicherung einschließlich der Alterssicherung der Landwirte können in Anspruch genommen werden:

1. in der gesetzlichen Rentenversicherung: a) Heilbehandlung, Leistungen zur Teilhabe am Arbeitsleben und andere Leistunaur Erhaltung, Besserung und Wiederherstellung der Erwerbsfähigkeit einschließlich wirtschaftlicher Hilfen,

b) Renten wegen Alters, Renten wegen verminderter Erwerbsfähigkeit und Knappschaftsausgleichsleistung,

c) Renten wegen Todes,

e) Zuschüsse zu den Aufwendungen für die Krankenversicherung,

e) Zuschüsse zu den Aufwendungen

2. in der Alterssicherung der Landwirte:

a) Heilbehandlung und andere Leistungen zur Erhaltung, Besserung und Wiederertllung der Erwerbsfähigkeit einschließlich Betriebs- oder Haushaltshilfe,

b) Renten wegen Erwerbsminderung und Alters,

c) Renten wegen Todes,

d) Beitragszuschüsse,

e) Betriebs- und Haushaltshilfe oder sonstige Leistungen zur Aufrechterhaltung des Unternehmens der Landwirtschaft.

(2) Zuständig sind ...

\section{Leistungen zur Rehabilitation und Teilhabe behinderter Menschen}

5 29. (1) Nach dem Recht der Rehabilitation und Teilhabe behinderter Menschen können in Anspruch genommen werden

1. Leistungen zur medizinischen Rehabilitation, insbesondere

a) Frühförderung behinderter und von Behinderung bedrohter Kinder,

b) ärztliche und zahnärztliche Behandlung,

c) Arznei- und Verbandmittel sowie Heilmittel einschließlich physikalischer, Sprachund Beschäftigungstherapie,

d) Körperersatzstücke, orthopädische und andere Hilfsmittel,

e) Belastungserprobung und Arbeitstherapie,

2. Leistungen zur Teilhabe am Arbeitsleben, insbesondere

a) Hilfen zum Erhalten oder Erlangen eines Arbeitsplatzes,

b) Berufsvorbereitung, berufliche Anpassung, Ausbildung und Weiterbildung,

c) sonstige Hilfen zur Förderung der Teilhabe am Arbeitsleben,

3. Leistungen zur Teilhabe am Leben in der Gemeinschaft, insbesondere Hilfen

a) zur Entwicklung der geistigen und körperlichen Fähigkeiten vor Beginn der Schulpflicht,

b) zur angemessenen Schulbildung,

c) zur heilpädagogischen Förderung,

d) zum Erwerb praktischer Kenntnisse und Fähigkeiten,

e) zur Ausübung einer angemessenen Tätigkeit, soweit Leistungen zur Teilhabe am Arbeitsleben nicht möglich sind,

f) zur Förderung der Verständigung mit der Umwelt,

g) zur Freizeitgestaltung und sonstigen Teilhabe am gesellschaftlichen Leben,

4. unterhaltssichernde und andere ergänzende Leistungen, insbesondere

a) Krankengeld, Versorgungskrankengeld, Verletztengeld, Übergangsgeld, Ausbildungsgeld oder Unterhaltsbeihilfe,

b) Beiträge zur gesetzlichen Kranken-, Unfall-, Renten- und Pflegeversicherung sowie zur Bundesagentur für Arbeit,

c) Reisekosten,

d) Haushalts- oder Betriebshilfe und Kinderbetreuungskosten,

e) Rehabilitationssport und Funktionstraining 
5.besondere Leistungen und sonstige Hilfen zur Teilhabe schwerbehinderter Menschen am Leben in der Gesellschaft, insbesondere am Arbeitsleben.

(2) Zuständig sind die in den $\$ \$ 19$ bis 24,27 und 28 genannten Leistungsträger und die Integrationsämter.

\section{Vorläufige Leistungen}

5 43. (1) Besteht ein Anspruch auf Sozialleistungen und ist zwischen mehreren Leistungsträgern streitig, wer zur Leistung verpflichtet ist, kann der unter ihnen zuers angegangene Leistungsträger vorläufig Leistungen erbringen, deren Umfang er nach pflichtgemäßem Ermessen bestimmt. Er hat Leistungen nach Satz 1 zu erbringen, wenn der Berechtigte es beantragt; die vorläufigen Leistungen beginnen spätestens nach Ablauf eines Kalendermonats nach Eingang des Antrags.

(2) Für die Leistungen nach Absatz 1 gilt $\$ 42$ Abs. 2 und 3 entsprechend. Ein Erstattungsanspruch gegen den Empfänger steht nur dem zur Leistung verpflichteten Leistungsträger $\mathrm{zu}$.

\section{Heilbehandlung}

\$ 63. Wer wegen Krankheit oder Behinderung Sozialleistungen beantragt oder erhält, soll sich auf Verlangen des zuständigen Leistungsträgers einer Heilbehandlung unterziehen, wenn zu erwarten ist, daß sie eine Besserung seines Gesundheitszustands herbeiführen oder eine Verschlechterung verhindern wird.

\section{Grenzen der Mitwirkung}

\$ 65. (1) Die Mitwirkungspflichten nach den $\$ \$ 60$ bis 64 bestehen nicht, soweit

1. ihre Erfüllung nicht in einem angemessenen Verhältnis zu der in Anspruch genommenen Sozialleistung oder ihrer Erstattung steht oder

2. ihre Erfüllung dem Betroffenen aus einem wichtigen Grund nicht zugemutet werden kann oder

3. der Leistungsträger sich durch einen geringeren Aufwand als der Antragsteller oder Leistungsberechtigte die erforderlichen Kenntnisse selbst beschaffen kann.

2) Behandlungen und Untersuchungen,

1. bei denen im Einzelfall ein Schaden für Leben oder Gesundheit nicht mit hoher Wahrscheinlichkeit ausgeschlossen werden kann,

2. die mit erheblichen Schmerzen verbunden sind oder

3. die einen erheblichen Eingriff in die körperliche Unversehrtheit bedeuten,

können abgelehnt werden.

(3) Angaben, die dem Antragsteller, dem Leistungsberechtigten oder ihnen nahestehende Personen ( $\$ 383$ Abs. 1 Nr. 1 bis 3 der Zivilprozeßordnung) die Gefahr zuziehen würden, wegen einer Straftat oder einer Ordnungswidrigkeit verfolgt zu werden, können verweigert werden.

\section{Sozialgesetzbuch (SGB) Fünftes Buch (V) Gesetzliche Krankenversicherung}

\section{Solidarität und Eigenverantwortung}

\$1. Die Krankenversicherung als Solidargemeinschaft hat die Aufgabe, die Gesundheit der Versicherten zu erhalten, wiederherzustellen oder ihren Gesundheitszustand zu bessern. Die Versicherten sind für ihre Gesundheit mitverantwortlich; sie sollen durch eine gesundheitsbewußte Lebensführung, durch frühzeitige Beteiligung an gesundheitlichen Vorsorgemaßnahmen sowie durch aktive Mitwirkung an Krankenbehandlung und
Rehabilitation dazu beitragen, den Eintritt von Krankheit und Behinderung zu vermeiden oder ihre Folgen zu überwinden. Die Krankenkassen haben den Versicherten dabei durch Aufklärung, Beratung und Leistungen zu helfen und auf gesunde Lebensverhältnisse hinzuwirken.

\section{Leistungsarten}

\$ 11. (1) Versicherte haben nach den folgenden Vorschriften Anspruch auf Leistungen

1. (weggefallen)

2. zur Verhütung von Krankheiten und von deren Verschlimmerung sowie zur Empfängnisverhütung, bei Sterilisation und bei Schwangerschaftsabbruch ( $\$ 20$ bis $24 \mathrm{~b}$ ),

3. zur Früherkennung von Krankheiten ( $\$ \$ 25$ und 26)

4. zur Behandlung einer Krankheit ( $\$ 27$ bis 52 ),

5. es Persönlichen Budgets nach $\$ 17$ Abs. 2 bis 4 des Neunten Buches.

(2) Versicherte haben auch Anspruch auf Leistungen zur medizinischen Rehabilitation sowie auf unterhaltssichernde und andere ergänzende Leistungen, die notwendig sind, um eine Behinderung oder Pflegebedürftigkeit abzuwenden, zu beseitigen, zu mindern, auszugleichen, ihre Verschlimmerung zu verhüten oder ihre Folgen zu mildern. Leistungen der aktivierenden Pflege nach Eintritt von Pflegebedürftigkeit werden von den Pflegekassen erbracht. Die Leistungen nach Satz 1 werden unter Beachtung des Neunten Buches erbracht, soweit in diesem Buch nichts anderes bestimmt ist.

(3) Bei stationärer Behandlung..... notwendige Mitaufnahme einer Begleitperson des Versicherten.

(4) Versicherte haben Anspruch auf ein Versorgungsmanagement insbesondere zur Lösung von Problemen beim Übergang in die verschiedenen Versorgungsbereiche. Die betroffenen Leistungserbringer sorgen für eine sachgerechte Anschlussversorgung des Versicherten und übermitteln sich gegenseitig die erforderlichen Informationen. Sie sind zur Erfüllung dieser Aufgabe von den Krankenkassen zu unterstützen. In das Versorgungsmanagement sind die Pflegeeinrichtungen einzubeziehen; dabei ist eine enge $\mathrm{Zu}$ sammenarbeit mit Pflegeberatern und Pflegeberaterinnen nach $\$ 7$ a des Elften Buches zu gewährleisten. Das Versorgungsmanagement und eine dazu erforderliche Übermittlung gewährleisten. Das Versorgungsmanagement und eine dazu erforderliche Ubermittlung
von Daten darf nur mit Einwilligung und nach vorheriger Information des Versicherten erfolgen....

(5) Auf Leistungen besteht kein Anspruch, wenn sie als Folge eines Arbeitsunfalls oder einer Berufskrankheit im Sinne der gesetzlichen Unfallversicherung zu erbringen sind.

\section{Medizinische Vorsorgeleistungen}

§ 23. (1) Versicherte haben Anspruch auf ärztliche Behandlung und Versorgung mit Arznei-, Verband-, Heil- und Hilfsmitteln, wenn diese notwendig sind,

1 eine Schwächung der Gesundheit, die in absehbarer Zeit voraussichtlich zu einer Krankheit führen würde, zu beseitigen,

2. einer Gefährdung der gesundheitlichen Entwicklung eines Kindes entgegenzuwirken,

3. Krankheiten zu verhüten oder deren Verschlimmerung zu vermeiden oder

4. Pflegebedürftigkeit zu vermeiden.

(2) Reichen bei Versicherten die Leistungen nach Absatz 1 nicht aus, kann die Krankenkasse aus medizinischen Gründen erforderliche ambulante Vorsorgeleistungen in anerkannten Kurorten erbringen ...

(3) $\ldots$ 
(4) Reichen bei Versicherten die Leistungen nach Absatz 1 und 2 nicht aus, kann die Krankenkasse Behandlung mit Unterkunft und Verpflegung in einer Vorsorgeeinrichtung erbringen, mit der ein Vertrag nach $\$ 111$ besteht. ...

(5) Die Krankenkasse bestimmt nach den medizinischen Erfordernissen des Einzelfalls Art, Dauer, Umfang, Beginn und Durchführung der Leistungen nach Absatz 4 sowie die Vorsorgeeinrichtung nach pflichtgemäßem Ermessen. Leistungen nach Absatz 4 sollen für längstens drei Wochen erbracht werden, es sei denn, eine Verlängerung der Leistung ist aus medizinischen Gründen dringend erforderlich ... Leistungen nach Absatz 2 können nicht vor Ablauf von drei, Leistungen nach Absatz 4 können nicht vor Ablauf von vier Jahren nach Durchführung solcher oder ähnlicher Leistungen erbracht werden, deren Kosten auf Grund öffentlich-rechtlicher Vorschriften getragen oder bezuschusst worden sind, es sei denn, eine vorzeitige Leistung ist aus medizinischen Gründen dringend erforderlich.

(6) ...

(7) Medizinisch notwendige stationäre Vorsorgemaßnahmen für versicherte Kinder, die das 14. Lebensjahr noch nicht vollendet haben, sollen in der Regel für vier bi sechs Wochen erbracht werden.

\section{Medizinische Vorsorge für Mütter und Väter}

\$24. (1) Versicherte haben unter den in $\$ 23$ Abs. 1 genannten Voraussetzungen Anspruch auf aus medizinischen Gründen erforderliche Vorsorgeleistungen in einer Einrichtung des Müttergenesungswerks oder einer gleichartigen Einrichtung; die Leistung kann in Form einer Mutter-Kind-Maßnahme erbracht werden. Satz 1 gilt auch für VaterKind-Maßnahmen in dafür geeigneten Einrichtungen. ...

(3) $\ldots$

\section{Krankenbehandlung}

$\$ 27$. (1) Versicherte haben Anspruch auf Krankenbehandlung, wenn sie notwendig ist, um eine Krankheit zu erkennen, zu heilen, ihre Verschlimmerung zu verhüten oder Krankheitsbeschwerden zu lindern. Die Krankenbehandlung umfaßt

1. Ärztliche Behandlung einschließlich Psychotherapie als ärztliche und psychotherapeutische Behandlung

2. zahnärztliche Behandlung, onen,

2a. Versorgung mit Zahnersatz einschließlich Zahnkronen und Suprakonstrukti-

3. Versorgung mit Arznei-, Verband-, Heil- und Hilfsmitteln,

4. häusliche Krankenpflege und Haushaltshilfe,

5. Krankenhausbehandlung,

6. Leistungen zur medizinischen Rehabilitation und ergänzende Leistungen.

Bei der Krankenbehandlung ist den besonderen Bedürfnissen psychisch Kranker Rechnung zu tragen, insbesondere bei der Versorgung mit Heilmitteln und bei der medizinischen Rehabilitation. Zur Krankenbehandlung gehören auch Leistungen zur Herstellung der Zeugungs- oder Empfängnisfähigkeit, wenn diese Fähigkeit nicht vorhanden war oder durch Krankheit oder wegen einer durch Krankheit erforderlichen Sterilisation verlorengegangen war.

(2) Versicherte, die sich nur vorübergehend im Inland aufhalten, Ausländer, ...

\section{Ärztliche und zahnärztliche Behandlung}

5 28. (1) Die ärztliche Behandlung umfaßt die Tätigkeit des Arztes, die zur Verhütung, Früherkennung und Behandlung von Krankheiten nach den Regeln der ärztlichen
Kunst ausreichend und zweckmäßig ist. Zur ärztlichen Behandlung gehört auch die Hilfeleistung anderer Personen, die von dem Arzt angeordnet und von ihm zu verantworten ist.

(2) Die zahnärztliche Behandlung umfaßt die Tätigkeit des Zahnarztes, die zur Verhütung, Früherkennung und Behandlung von Zahn-, Mund- und Kieferkrankheiten nach den Regeln der zahnärztlichen Kunst ausreichend und zweckmäßig ist; sie umfasst auch

(3) Die psychotherapeutische Behandlung einer Krankheit wird durch Psychologische Psychotherapeuten und Kinder- und Jugendlichenpsychotherapeuten (Psychotherapeuten) ... durchgeführt ...

(4) ... Zuzahlung ...

\section{Krankenhausbehandlung}

5 39. (1) Die Krankenhausbehandlung wird vollstationär, teilstationär, vor- und nachstationär ( $\$ 115 a)$ sowie ambulant $(\$ 115 b)$ erbracht. Versicherte haben Anspruch auf vollstationäre Behandlung in einem zugelassenen Krankenhaus $(\$ 108)$, wenn die Aufnahme nach Prüfung durch das Krankenhaus erforderlich ist, weil das Behandlungsziel nicht durch teilstationäre, vor- und nachstationäre oder ambulante Behandlung einschließlich häuslicher Krankenpflege erreicht werden kann. Die Krankenhausbehandlung umfaßt im Rahmen des Versorgungsauftrags des Krankenhauses alle Leistungen, die im Einzelfall nach Art und Schwere der Krankheit für die medizinische Versorgung der Versicherten im Krankenhaus notwendig sind, insbesondere ärztliche Behandlung ( $\$ 28$ Abs. 1), Krankenpflege, Versorgung mit Arznei-, Heil- und Hilfsmitteln, Unterkunft und Verpflegung; die akutstationäre Behandlung umfasst auch die im Einzelfall erforderlichen und zum frühestmöglichen Zeitpunkt einsetzenden Leistungen zur Frührehabilitation.

(2) ... Mehrkosten ...

(3) $\ldots$

(4)

\section{Leistungen zur medizinischen Rehabilitation}

5 40. (1) Reicht bei Versicherten eine ambulante Krankenbehandlung nicht aus, um die in $\$ 11$ Abs. 2 beschriebenen Ziele zu erreichen, erbringt die Krankenkasse aus medizinischen Gründen erforderliche ambulante Rehabilitationsleistungen in Rehabilitationseinrichtungen, für die ein Versorgungsvertrag nach $\$ 111$ besteht, oder, soweit dies für eine bedarfsgerechte, leistungsfähige und wirtschaftliche Versorgung der Versicherten mit medizinischen Leistungen ambulanter Rehabilitation erforderlich ist, durch wohnortnahe Einrichtungen. Leistungen nach Satz 1 sind auch in stationären Pflegeeinrichtungen nach $\$ 72$ Abs. 1 des Elften Buches zu erbringen.

(2) Reicht die Leistung nach Absatz 1 nicht aus, erbringt die Krankenkasse stationäre Rehabilitation mit Unterkunft und Verpflegung in einer nach $\$ 20$ Abs. 2a des Neunten Buches zertifizierten Rehabilitationseinrichtung, mit der ein Vertrag nach $\$ 111$ besteht. Wählt der Versicherte eine andere zertifizierte Einrichtung, mit der kein Versorgungsvertrag nach $\$ 111$ besteht, so hat er die dadurch entstehenden Mehrkosten zu tragen.

(3) Die Krankenkasse bestimmt nach den medizinischen Erfordernissen des Einzelfalls Art, Dauer, Umfang, Beginn und Durchführung der Leistungen nach den Absätzen 1 und 2 sowie die Rehabilitationseinrichtung nach pflichtgemäßem Ermessen. Leistungen nach Absatz 1 sollen für längstens 20 Behandlungstage, Leistungen nach Absatz 2 für längstens drei Wochen erbracht werden, es sei denn, eine Verlängerung der Leistung ist aus medizinischen Gründen dringend erforderlich. Satz 2 gilt nicht, soweit ... Leitlinien Indikationen festgelegt und ... jeweils eine Regeldauer zugeordnet ...; von dieser Regel- 
dauer kann nur abgewichen werden, wenn dies aus dringenden medizinischen Gründen im Einzelfall erforderlich ist. Leistungen nach den Absätzen 1 und 2 können nicht vor Ablauf von vier Jahren nach Durchführung solcher oder ähnlicher Leistungen erbracht schusst worden sind, es sei denn, eine vorzeitige Leistung ist aus medizinischen Gründen dringend erforderlich. $\$ 23$ Abs. 7 gilt entsprechend. Die Krankenkasse zahlt der Pflegekasse einen Betrag in Höhe von 3.072 Euro für pflegebedürftige Versicherte, für die innerhalb von sechs Monaten nach Antragstellung keine notwendigen Leistungen zur medizinischen Rehabilitation erbracht worden sind. Satz 6 gilt nicht, wenn die Krankenkasse die fehlende Leistungserbringung nicht zu vertreten hat. Die Krankenkasse berichtet ihrer die fehlende Leistungserbringung nicht zu vertre

(4) Leistungen nach den Absätzen 1 und 2 werden nur erbracht, wenn nach den für andere Träger der Sozialversicherung geltenden Vorschriften mit Ausnahme des $\$ 31$ des Sechsten Buches solche Leistungen nicht erbracht werden können.

(5) ...

(6) Versicherte, die das achtzehnte Lebensjahr vollendet haben und eine Leistung nach Absatz 1 oder 2 in Anspruch nehmen, deren unmittelbarer Anschluß an eine Krankenhausbehandlung medizinisch notwendig ist (Anschlußrehabilitation), zahlen den sich nach $\$ 61$ Satz 2 ergebenden Betrag für längstens 28 Tage je Kalenderjahr an die Einrichtung; als unmittelbar gilt der Anschluß auch, wenn die Maßnahme innerhalb von $14 \mathrm{Ta}$ cen berinnt, Zalenderjahres bereits an einen Träger der gesetzlichen Rentenversicherung geleistete kalendertägliche Zahlung nach $\$ 32$ Abs. 1 Satz 2 des Sechsten Buches sowie die nach $\$ 39$ Abs. 4 geleistete Zahlung sind auf die Zahlung nach Satz 2 anzurechnen. Die Zahlungen sind an die Krankenkasse weiterzuleiten.

(7) Der Spitzenverband Bund der Krankenkassen legt unter Beteiligung der Arbeitsgemeinschaft nach $\$ 282$ (Medizinischer Dienst der Spitzenverbände der Krankenkassen) Indikationen fest, bei denen für eine medizinisch notwendige Leistung nach Absatz 2 die Zuzahlung nach Absatz 6 Satz 1 Anwendung findet, ohne daß es sich um Anschlußrehabilitation handelt. Vor der Festlegung der Indikationen ist den für die Wahrnehmung der Interessen der stationären Rehabilitation auf Bundesebene maßgebenden Organisationen Gelegenheit zur Stellungnahme zu geben; die Stellungnahmen sind in die Entscheidung einzubeziehen.

\section{Medizinische Rehabilitation für Mütter und Väter}

\$ 41. (1) Versicherte haben unter den in $\$ 27$ Abs. 1 genannten Voraussetzungen Anspruch auf aus medizinischen Gründen erforderliche Rehabilitationsleistungen in eine Einrichtung des Müttergenesungswerks oder einer gleichartigen Einrichtung; die Leistung kann in Form einer Mutter-Kind-Maßnahme erbracht werden. Satz 1 gilt auch für VaterKind-Maßnahmen in dafür geeigneten Finrichtungen. Rehabilitationsleistungen nach den Sätzen 1 und 2 werden in Einrichtungen erbracht, mit denen ein Versorgungsvertrag nach $\$ 11$ la besteht. $\$ 40$ Abs. 2 Satz 1 und 2 gilt nicht; $\$ 40$ Abs. 2 Satz 3 gilt entsprechend.

(2) $\$ 40$ Abs. 3 und 4 gilt entsprechend.

(3) ...

\section{Ergänzende Leistungen zur Rehabilitation}

§ 43. (1) Die Krankenkasse kann neben den Leistungen, die nach $\$ 44$ Abs. 1 Nr. 2 bis 6 sowie nach $\$ \$ 53$ und 54 des Neunten Buches als ergänzende Leistungen zu erbringen sind, 1.solche Leistungen zur Rehabilitation ganz oder teilweise erbringen oder fördern, die unter Berücksichtigung von Art oder Schwere der Behinderung erforderlich sind, um das Ziel der Rehabilitation zu erreichen oder zu sichern, aber nicht zu den Leistungen zur Teilhabe am Arbeitsleben oder den Leistungen zur allgemeinen sozialen Eingliederung gehören,

2.wirksame und effiziente Patientenschulungsmaßnahmen für chronisch Kranke erbringen; Angehörige und ständige Betreuungspersonen sind einzubeziehen, wenn dies aus medizinischen Gründen erforderlich ist,

wenn zuletzt die Krankenkasse Krankenbehandlung geleistet hat oder leistet.

(2) Die Krankenkasse erbringt aus medizinischen Gründen in unmittelbarem Anschluss an eine Krankenhausbehandlung nach $\$ 39$ Abs. 1 oder stationäre Rehabilitation erforderliche sozialmedizinische Nachsorgemaßnahmen für chronisch kranke oder schwerstkranke Kinder und Jugendliche, die das 14. Lebensjahr, in besonders schwerwiegenden Fällen das 18. Lebensjahr, noch nicht vollendet haben, wenn die Nachsorge wegen der Art, Schwere und Dauer der Erkrankung notwendig ist, um den stationären Aufenthal zu verkürzen oder die anschließende ambulante ärztliche Behandlung zu sichern. Die Nachsorgemaßnahmen umfassen die im Einzelfall erforderliche Koordinierung der verordneten Leistungen sowie Anleitung und Motivation zu deren Inanspruchnahme. Angehörige und ständige Betreuungspersonen sind einzubeziehen, wenn dies aus medizinischen Gründen erforderlich ist. Der Spitzenverband Bund der Krankenkassen bestimmt das Nähere zu den Voraussetzungen sowie zu Inhalt und Qualität der Nachsorgemaßnahmen.

\section{Versorgungsverträge mit Vorsorge- oder Rehabilitationseinrichtungen}

$\$$ 111. (1) Die Krankenkassen dürfen medizinische Leistungen zur Vorsorge $(\$ 23$ Abs 4) oder Leistungen zur medizinischen Rehabilitation einschließlich der Anschluß heilbehandlung $(\$ 40)$, die eine stationäre Behandlung, aber keine Krankenhausbehandlung erfordern, nur in Vorsorge- oder Rehabilitationseinrichtungen erbringen lassen, mit denen ein Versorgungsvertrag nach Absatz 2 besteht.

(2) Die Landesverbände der Krankenkassen und die Ersatzkassen gemeinsam schlieBen mit Wirkung für ihre Mitgliedskassen einheitliche Versorgungsverträge über die Durchführung der in Absatz 1 genannten Leistungen mit Vorsorge- oder Rehabilitationseinrichtungen, die

1.die Anforderungen des $\$ 107$ Abs. 2 erfüllen und

2. für eine bedarfsgerechte, leistungsfähige und wirtschaftliche Versorgung der Versicherten ihrer Mitgliedskassen mit stationären medizinischen Leistungen zur Vorsorge oder Leistungen zur medizinischen Rehabilitation einschließlich der Anschlußheilbehandlung notwendig sind. ...

(3) ...

(4) Mit dem Versorgungsvertrag wird die Vorsorge- oder Rehabilitationseinrichtung für die Dauer des Vertrages zur Versorgung der Versicherten mit stationären medizinischen Leistungen zur Vorsorge oder Rehabilitation zugelassen. ...

(5) ... Vergütungen für die in Absatz 1 genannten Leistungen ...

(6) ...

\section{Sozialgesetzbuch (SGB) Sechstes Buch (VI) Gesetzliche Rentenversicherung \\ Aufgabe der Leistungen zur Teilhabe}

\$ 9. (1) Die Rentenversicherung erbringt Leistungen zur medizinischen Rehabilitation, Leistungen zur Teilhabe am Arbeitsleben sowie ergänzende Leistungen, um 
1.den Auswirkungen einer Krankheit oder einer körperlichen, geistigen oder seelischen Behinderung auf die Erwerbsfähigkeit der Versicherten entgegenzuwirken oder sie zu überwinden und

2.dadurch Beeinträchtigungen der Erwerbsfähigkeit der Versicherten oder ihr vorzeitiges Ausscheiden aus dem Erwerbsleben zu verhindern oder sie möglichst dauerhaft in das Erwerbsleben wiedereinzugliedern.

Die Leistungen zur Teilhabe haben Vorrang vor Rentenleistungen, die bei erfolgreichen Leistungen zur Teilhabe nicht oder voraussichtlich erst zu einem späteren Zeitpunkt zu erbringen sind.

(2) Die Leistungen nach Absatz 1 können erbracht werden, wenn die persönlichen und versicherungsrechtlichen Voraussetzungen dafür erfüllt sind.

\section{Persönliche Voraussetzungen}

\$ 10. (1) Für Leistungen zur Teilhabe haben Versicherte die persönlichen Voraussetzungen erfüllt,

1. deren Erwerbsfähigkeit wegen Krankheit oder körperlicher, geistiger oder seelischer Behinderung erheblich gefährdet oder gemindert ist und

2. bei denen voraussichtlich

a) bei erheblicher Gefährdung der Erwerbsfähigkeit eine Minderung der Erwerbsfähigkeit durch Leistungen zur medizinischen Rehabilitation oder zur Teilhabe am Arbeitsleben abgewendet werden kann

b) bei geminderter Erwerbsfähigkeit diese durch Leistungen zur medizinischen Rehabilitation oder zur Teilhabe am Arbeitsleben wesentlich gebessert oder wiederhergestellt oder hierdurch deren wesentliche Verschlechterung abgewendet werden kann,

c) bei teilweiser Erwerbsminderung ohne Aussicht auf eine wesentliche Besserung der Erwerbsfähigkeit der Arbeitsplatz durch Leistungen zur Teilhabe am Arbeitsleben erhalten werden kann

(2) Für Leistungen zur Teilhabe haben auch Versicherte die persönlichen Voraussetzungen erfüllt,

1. die im Bergbau vermindert berufsfähig sind und bei denen voraussichtlich durch die Leistungen die Erwerbsfähigkeit wesentlich gebessert oder wiederhergestellt werden kann oder

2. bei denen der Eintritt von im Bergbau verminderter Berufsfähigkeit droht und bei denen voraussichtlich durch die Leistungen der Eintritt der im Bergbau verminderten Berufsfähigkeit abgewendet werden kann.

\section{Versicherungsrechtliche Voraussetzungen}

5 11. (1) Für Leistungen zur Teilhabe haben Versicherte die versicherungsrechtlichen Voraussetzungen erfullt, die bei Antragstellung

1. die Wartezeit von 15 Jahren erfüllt haben ode

2. eine Rente wegen verminderter Erwerbsfähigkeit beziehen

(2) Für die Leistungen zur medizinischen Rehabilitation haben Versicherte die versicherungsrechtlichen Voraussetzungen auch erfüllt, die

1. in den letzten zwei Jahren vor der Antragstellung sechs Kalendermonate mit Pflichtbeiträgen für eine versicherte Beschäftigung oder Tätigkeit haben,

2. innerhalb von zwei Jahren nach Beendigung einer Ausbildung eine versicherte Beschäftigung oder selbständige Tätigkeit aufgenommen und bis zum Antrag ausgeübt haben oder nach einer solchen Beschäftigung oder Tätigkeit bis zum Antrag arbeitsunfähig oder arbeitslos gewesen sind oder
3. vermindert erwerbsfähig sind oder bei denen dies in absehbarer Zeit zu erwarten ist, wenn sie die allgemeine Wartezeit erfüllt haben.

$\$ 55$ Abs. 2 ist entsprechend anzuwenden.

(2a) Leistungen zur Teilhabe am Arbeitsleben werden an Versicherte auch erbracht

1. wenn ohne diese Leistungen Rente wegen verminderter Erwerbsfähigkeit zu leisten wäre oder

2. wenn sie für eine voraussichtlich erfolgreiche Rehabilitation unmittelbar im Anschluss an Leistungen zur medizinischen Rehabilitation der Träger der Rentenversicherung erforderlich sind.

(3) Die versicherungsrechtlichen Voraussetzungen haben auch überlebende Ehegatten erfüllt, die Anspruch auf große Witwenrente oder große Witwerrente wegen verminderter Erwerbsfähigkeit haben. Sie gelten für die Vorschriften dieses Abschnitts als Versicherte.

\section{Ausschluss von Leistungen}

$\$$ 12. (1) Leistungen zur Teilhabe werden nicht für Versicherte erbracht, die 1. wegen eines Arbeitsunfalls, einer Berufskrankheit, einer Schädigung im Sinne des sozialen Entschädigungsrechts oder wegen eines Einsatzunfalls, der Ansprüche nach dem Einsatz-Weiterverwendungsgesetz begründet, gleichartige Leistungen eines anderen $\mathrm{Re}-$ habilitationsträgers oder Leistungen zur Eingliederung nach dem Einsatz-Weiterverwendungsgesetz erhalten können,

2. eine Rente wegen Alters von wenigstens zwei Dritteln der Vollrente beziehen oder beantragt haben,

3. eine Beschäftigung ausüben, aus der ihnen nach beamtenrechtlichen oder entsprechenden Vorschriften Anwartschaft auf Versorgung gewährleistet ist,

4. als Bezieher einer Versorgung wegen Erreichens einer Altersgrenze versicherungsfrei sind,

4a. eine Leistung beziehen, die regelmäßig bis zum Beginn einer Rente wegen Alters gezahlt wird, oder

5... Untersuchungshaft ... Vollzug einer Freiheitsstrafe ... freiheitsentziehenden Maßregel der Besserung und Sicherung ...

(2) Leistungen zur medizinischen Rehabilitation werden nicht vor Ablauf von vie Jahren nach Durchführung solcher oder ähnlicher Leistungen zur Rehabilitation erbracht, deren Kosten aufgrund öffentlich-rechtlicher Vorschriften getragen oder bezuschuss worden sind. Dies gilt nicht, wenn vorzeitige Leistungen aus gesundheitlichen Gründen dringend erforderlich sind.

\section{Leistungsumfang}

§ 13. (1) Der Träger der Rentenversicherung bestimmt im Einzelfall unter Beachtung der Grundsätze der Wirtschaftlichkeit und Sparsamkeit Art, Dauer, Umfang, Beginn und Durchführung dieser Leistungen sowie die Rehabilitationseinrichtung nach pflichtgemäßem Ermessen. Die Leistungen können auf Antrag auch als Teil eines trägerübergreifenden Persönlichen Budgets erbracht werden; $\$ 17$ Abs. 2 bis 4 des Neunten Buches in Verbindung mit der Budgetverordnung und $\$ 159$ des Neunten Buches finden Anwendung

(2) Der Träger der Rentenversicherung erbringt nicht

1. Leistungen zur medizinischen Rehabilitation in der Phase akuter Behandlungsbedürftigkeit einer Krankheit, es sei denn, die Behandlungsbedürftigkeit tritt während der Ausführung von Leistungen zur medizinischen Rehabilitation ein,

2. Leistungen zur medizinischen Rehabilitation anstelle einer sonst erforderlichen Krankenhausbehandlung, 
3. Leistungen zur medizinischen Rehabilitation, die dem allgemein anerkannten Stand medizinischer Erkenntnisse nicht entsprechen.

(3) Der Träger der Rentenversicherung erbringt nach Absatz 2 Nr. 1 im Benehmen mit dem Träger der Krankenversicherung für diesen Krankenbehandlung und Leistungen bei Schwangerschaft und Mutterschaft. Der Träger der Rentenversicherung kann von dem Träger der Krankenversicherung Erstattung der hierauf entfallenden Aufwendungen verlangen.

(4) Die Träger der Rentenversicherung vereinbaren mit den Spitzenverbänden der Krankenkassen gemeinsam und einheitlich im Benehmen mit dem Bundesministerium für Arbeit und Soziales Näheres zur Durchführung von Absatz 2 Nr. 1 und 2.

\section{Leistungen zur medizinischen Rehabilitation}

5 15. (1) Die Träger der Rentenversicherung erbringen im Rahmen von Leistungen zur medizinischen Rehabilitation Leistungen nach den $\$ \$ 26$ bis 31 des Neunten Buches, ausgenommen Leistungen nach $\$ 26$ Abs. 2 Nr. 2 und $\$ 30$ des Neunten Buches. Zahnärztliche Behandlung einschließlich der Versorgung mit Zahnersatz wird nur erbracht, wenn sie unmittelbar und gezielt zur wesentlichen Besserung oder Wiederherstellung de Erwerbsfähigkeit, insbesondere zur Ausübung des bisherigen Berufs, erforderlich und soweit sie nicht als Leistung der Krankenversicherung oder als Hilfe nach dem Fünften Kapitel des Zwölften Buches zu erbringen ist.

(2) Die stationären Leistungen zur medizinischen Rehabilitation werden einschließlich der erforderlichen Unterkunft und Verpflegung in Einrichtungen erbracht, die unter ständiger ärztlicher Verantwortung und unter Mitwirkung von besonders geschultem Personal entweder von dem Träger der Rentenversicherung selbst betrieben werden ode mit denen ein Vertrag nach $\$ 21$ des Neunten Buches besteht. Die Einrichtung braucht nicht unter ständiger ärztlicher Verantwortung zu stehen, wenn die Art der Behandlung dies nicht erfordert. Die Leistungen der Einrichtungen der medizinischen Rehabilitation müssen nach Art oder Schwere der Erkrankung erforderlich sein.

(3) Die stationären Leistungen zur medizinischen Rehabilitation sollen für längsten drei Wochen erbracht werden. Sie können für einen längeren Zeitraum erbracht werden, wenn dies erforderlich ist, um das Rehabilitationsziel zu erreichen.

\section{Ergänzende Leistungen}

§ 28. Die Leistungen zur Teilhabe werden außer durch das Übergangsgeld ergänzt durch die Leistungen nach $\$ 44$ Abs. 1 Nr. 2 bis 6 und Abs. 2 sowie nach den $\$ \$ 53$ und 54 des Neunten Buches.

\section{Sonstige Leistungen}

\$ 31. (1) Als sonstige Leistungen zur Teilhabe können erbracht werden:

1. Leistungen zur Eingliederung von Versicherten in das Erwerbsleben, insbesondere nachgehende Leistungen zur Sicherung des Erfolges der Leistungen zur Teilhabe,

2. stationäre medizinische Leistungen zur Sicherung der Erwerbsfähigkeit für Versicherte, die eine besonders gesundheitsgefährdende, ihre Erwerbsfähigkeit ungünstig beeinflussende Beschäftigung ausüben,

3. Nach- und Festigungskuren wegen Geschwulsterkrankungen für Versicherte, Bezieher einer Rente sowie ihre Angehörigen,

4. stationäre Heilbehandlung für Kinder von Versicherten, Beziehern einer Rente wegen Alters, wegen verminderter Erwerbsfähigkeit oder für Bezieher einer Waisenrente, wenn hierdurch voraussichtlich eine erhebliche Gefährdung der Gesundheit beseitigt oder eine beeinträchtigte Gesundheit wesentlich gebessert oder wiederhergestellt werden kann,
5. Zuwendungen für Einrichtungen, die auf dem Gebiet der Rehabilitation forschen oder die Rehabilitation fördern.

Für Kinderheilbehandlungen findet $\$ 12$ Abs. 2 Anwendung.

(2) Die Leistungen nach Absatz 1 Satz 1 Nr. 1 setzen voraus, dass die persönlichen und versicherungsrechtlichen Voraussetzungen, die Leistungen nach Absatz 1 Satz 1 Nr. 2 und die Leistungen für Versicherte nach Absatz 1 Satz $1 \mathrm{Nr}$. 3, dass die versicherungsrechtlichen Voraussetzungen erfüllt sind, die Leistungen nach Absatz 1 Satz 1 Nr. 4, dass der Versicherte die versicherungsrechtlichen Voraussetzungen für Leistungen zur medizinischen Rehabilitation erfült. Sie werden nur auf Grund von Richtlinien der Deutschen inischen Rehabilitation erfillt Sie werden nur auf Grund von Richtlinien der Deuts racht die im Benehment mit dem Bundesministerium für Arbeit und Soziales erlassen werden.

(3) Die Aufwendungen für nichtstationäre Leistungen nach Absatz 1 Satz $1 \mathrm{Nr} .1$ sowie für sonstige Leistungen nach Absatz 1 Satz 1 Nr. 2, 4 und 5 dürfen im Kalenderjahr 7,5 vom Hundert der Haushaltsansätze für die Leistungen zur medizinischen Rehabilitation, die Leistungen zur Teilhabe am Arbeitsleben und die ergänzenden Leistungen nicht übersteigen.

\section{Zuzahlung bei Leistungen zur medizinischen Rehabilitation} und bei sonstigen Leistungen

5 32. (1) Versicherte, die das 18. Lebensjahr vollendet haben und stationäre Leistungen zur medizinischen Rehabilitation in Anspruch nehmen, zahlen für jeden Kalendertag dieser Leistungen den sich nach $\$ 40$ Abs. 5 des Fünften Buches ergebenden Betrag.

(2) Absatz 1 gilt auch für Versicherte oder Bezieher einer Rente, die das 18. Lebensjahr vollendet haben und für sich, ihre Ehegatten oder Lebenspartner sonstige stationäre Leistungen in Anspruch nehmen.

(3) $\ldots$
(4) $\ldots$
(5) $\ldots$

\section{Sozialgesetzbuch (SGB) Siebtes Buch (VII) Gesetzliche Unfallversicherung}

\section{Prävention, Rehabilitation, Entschädigung}

\$1. Aufgabe der Unfallversicherung ist es, nach Maßgabe der Vorschriften dieses

1. mit allen geeigneten Mitteln Arbeitsunfälle und Berufskrankheiten sowie arbeitsbedingte Gesundheitsgefahren zu verhüten,

2. nach Eintritt von Arbeitsunfällen oder Berufskrankheiten die Gesundheit und die Leistungsfähigkeit der Versicherten mit allen geeigneten Mitteln wiederherzustellen und sie oder ihre Hinterbliebenen durch Geldleistungen zu entschädigen.

\section{Arbeitsunfall}

5 8. (1) Arbeitsunfälle sind Unfälle von Versicherten infolge einer den Versicherungsschutz nach $\$ 2,3$ oder 6 begründenden Tätigkeit (versicherte Tätigkeit). Unfälle sind zeitlich begrenzte, von außen auf den Körper einwirkende Ereignisse, die zu einem Gesundheitsschaden oder zum Tod führen.

(2) Versicherte Tätigkeiten sind auch telbaren Weges nach und von dem Ort der Tätigkeit, 
2. das Zurücklegen des von einem unmittelbaren Weg nach und von dem Ort der Tätigkeit abweichenden Weges ...

3. das Zurücklegen des von einem unmittelbaren Weg nach und von dem Ort der Tätigkeit abweichenden Weges der Kinder ...

4. das Zurücklegen des mit der versicherten Tätigkeit zusammenhängenden Weges

5. das mit einer versicherten Tätigkeit zusammenhängende Verwahren, Befördern, Instandhalten und Erneuern eines Arbeitsgeräts oder einer Schutzausrüstung sowie deren Erstbeschaffung, wenn diese auf Veranlassung der Unternehmer erfolgt.

(3) Als Gesundheitsschaden gilt auch die Beschädigung oder der Verlust eines Hilfsmittels.

\section{Berufskrankheit}

5 9. (1) Berufskrankheiten sind Krankheiten, die die Bundesregierung durch Rechtsverordnung mit Zustimmung des Bundesrates als Berufskrankheiten bezeichnet und die Versicherte infolge einer den Versicherungsschutz nach $\$ 2,3$ oder 6 begründenden Tätigkeit erleiden. Die Bundesregierung wird ermächtigt, in der Rechtsverordnung solche Krankheiten als Berufskrankheiten zu bezeichnen,...

(2) Die Unfallversicherungsträger haben eine Krankheit, die nicht in der Rechtsverordnung bezeichnet ist oder bei der die dort bestimmten Voraussetzungen nicht vorliegen, wie eine Berufskrankheit als Versicherungsfall anzuerkennen, sofern im Zeitpunkt der Entscheidung nach neuen Erkenntnissen der medizinischen Wissenschaft die Voraussetzungen für eine Bezeichnung nach Absatz 1 Satz 2 erfüllt sind.

(3) Erkranken Versicherte, die infolge der besonderen Bedingungen ihrer versicherten Tätigkeit in erhöhtem Maße der Gefahr der Erkrankung an einer in der Rechtsverordnung nach Absatz 1 genannten Berufskrankheit ausgesetzt waren, an einer solchen Krankheit und können Anhaltspunkte für eine Verursachung außerhalb der versicherten Tätigkeit nicht festgestellt werden, wird vermutet, daß diese infolge der versicherten Tätigkeit verursacht worden ist.

(4) Setzt die Anerkennung einer Krankheit als Berufskrankheit die Unterlassung aller Tätigkeiten voraus, die für die Entstehung, die Verschlimmerung oder das Wiederaufleben der Krankheit ursächlich waren oder sein können, haben die Unfallversicherungsträger vor Unterlassung einer noch verrichteten gefährdenden Tätigkeit darüber zu entscheiden, ob die übrigen Voraussetzungen für die Anerkennung einer Berufskrankheit erfüllt sind.

(6) Die Bundesregierung regelt durch Rechtsverordnung mit Zustimmung des Bundesrates

1. Voraussetzungen, Art und Umfang von Leistungen zur Verhütung des Entstehens, der Verschlimmerung oder des Wiederauflebens von Berufskrankheiten,

2. die Mitwirkung der für den medizinischen Arbeitsschutz zuständigen Stellen bei der Feststellung von Berufskrankheiten sowie von Krankheiten, die nach Absatz 2 wie Berufskrankheiten zu entschädigen sind; ...

3....

(7) Die Unfallversicherungsträger haben die für den medizinischen Arbeitsschutz zuständige Stelle über den Ausgang des Berufskrankheitenverfahrens zu unterrichten, ...

(8) Die Unfallversicherungsträger ... Gewinnung neuer medizinisch-wissenschaftlicher Erkenntnisse ...

(9) Die für den medizinischen Arbeitsschutz zuständigen Stellen ... Feststellung von Berufskrankheiten

\section{Grundsatz}

\$26. (1) Versicherte haben nach Maßgabe der folgenden Vorschriften und unter Beachtung des Neunten Buches Anspruch auf Heilbehandlung einschließlich Leistungen zur medizinischen Rehabilitation, auf Leistungen zur Teilhabe am Arbeitsleben und am Leben in der Gemeinschaft, auf ergänzende Leistungen, auf Leistungen bei Pflegebedürftigkeit sowie auf Geldleistungen. Sie können einen Anspruch auf Ausführung der istungen durch ein Persönliches Budget nach $\$ 17$ Abs. 2 bis 4 des Neunten Buches in Verbindung mit der Budgetverordnung und $\$ 159$ des Neunten Buches haben; dies gilt im Rahmen des Anspruches auf Heilbehandlung nur für die Leistungen zur medizinischen Rehabilitation.

(2) Der Unfallversicherungsträger hat mit allen geeigneten Mitteln möglichst frühzeitig

1. den durch den Versicherungsfall verursachten Gesundheitsschaden zu beseitigen oder zu bessern, seine Verschlimmerung zu verhüten und seine Folgen zu mildern,

2. den Versicherten einen ihren Neigungen und Fähigkeiten entsprechenden Platz im Arbeitsleben zu sichern,

3. Hilfen zur Bewältigung der Anforderungen des täglichen Lebens und zur Teilhabe am Leben in der Gemeinschaft sowie zur Führung eines möglichst selbständigen Lebens unter Berücksichtigung von Art und Schwere des Gesundheitsschadens bereitzustellen,

4. ergänzende Leistungen zur Heilbehandlung und zu Leistungen zur Teilhabe am Arbeitsleben und am Leben in der Gemeinschaft zu erbringen,

5. Leistungen bei Pflegebedürftigkeit zu erbringen.

(3) Die Leistungen zur Heilbehandlung und zur Rehabilitation haben Vorrang vor Rentenleistungen.

(4) Qualität und Wirksamkeit der Leistungen zur Heilbehandlung und Teilhabe haben dem allgemein anerkannten Stand der medizinischen Erkenntnisse zu entsprechen und den medizinischen Fortschritt zu berücksichtigen. Sie werden als Dienst- und Sachleistungen zur Verfügung gestellt, soweit dieses oder das Neunte Buch keine Abweichungen vorsehen.

(5) Die Unfallversicherungsträger bestimmen im Einzelfall Art, Umfang und Durchführung der Heilbehandlung und der Leistungen zur Teilhabe sowie die Einrichtungen, die diese Leistungen erbringen, nach pflichtgemäßem Ermessen. Dabei prüfen sie auch, welche Leistungen geeignet und zumutbar sind, Pflegebedürftigkeit zu vermeiden, zu überwinden, zu mindern oder ihre Verschlimmerung zu verhüten.

\section{Umfang der Heilbehandlung}

$\$ 27$. (1) Die Heilbehandlung umfaßt insbesondere

1. Erstversorgung,

2. ärztliche Behandlung

3. zahnärztliche Behandlung einschließlich der Versorgung mit Zahnersatz,

4. Versorgung mit Arznei-, Verband-, Heil- und Hilfsmitteln,

5. häusliche Krankenpflege,

6. Behandlung in Krankenhäusern und Rehabilitationseinrichtungen,

7. Leistungen zur medizinischen Rehabilitation nach $\$ 26 \mathrm{Abs}$. $2 \mathrm{Nr} 1$ und 3 bis 7 und Abs. 3 des Neunten Buches.

(2) In den Fällen des $\$ 8$ Abs. 3 wird ein beschädigtes oder verlorengegangenes Hilfsmittel wiederhergestellt oder erneuert.

(3) Während einer aufgrund eines Gesetzes angeordneten Freiheitsentziehung wird Heilbehandlung erbracht, soweit Belange des Vollzugs nicht entgegenstehen. 


\section{Ärztliche und zahnärztliche Behandlung}

\$28. (1) Die ärztliche und zahnärztliche Behandlung wird von Ärzten oder Zahnärzten erbracht. Sind Hilfeleistungen anderer Personen erforderlich, dürfen sie nur erbrach werden, wenn sie vom Arzt oder Zahnarzt angeordnet und von ihm verantwortet werden.

(2) Die ärztliche Behandlung umfaßt die Tätigkeit der Ärzte, die nach den Regeln der ärztlichen Kunst erforderlich und zweckmäßig ist.

(3) Die zahnärztliche Behandlung umfaßt die Tätigkeit der Zahnärzte, die nach den Regeln der zahnärztlichen Kunst erforderlich und zweckmäßig ist.

(4) Bei Versicherungsfällen, für die wegen ihrer Art oder Schwere besondere unfallmedizinische Behandlung angezeigt ist, wird diese erbracht. Die freie Arztwahl kann insoweit eingeschränkt werden.

\section{Heilmittel}

§ 30. Heilmittel sind alle ärztlich verordneten Dienstleistungen, die einem Heilzweck dienen oder einen Heilerfolg sichern und nur von entsprechend ausgebildeten Personen erbracht werden dürfen. Hierzu gehören insbesondere Maßnahmen der physikalischen Therapie sowie der Sprach- und Beschäftigungstherapie.

\section{Hilfsmittel}

\$ 31. (1) Hilfsmittel sind alle ärztlich verordneten Sachen, die den Erfolg der Heilbehandlung sichern oder die Folgen von Gesundheitsschäden mildern oder ausgleichen Dazu gehören insbesondere Körperersatzstücke, orthopädische und andere Hilfsmittel

(2) Die Bundesregierung wird ermächtigt, durch Rechtsverordnung mit Zustimmung des Bundesrates die Ausstattung mit Körperersatzstücken, orthopädischen und anderen Hilfsmitteln zu regeln ... Das Nähere ... Verbände der Unfallversicherungsträger ... gemeinsame Richtlinien.

\section{Behandlung in Krankenhäusern und Rehabilitationseinrichtungen}

\$33. (1) Stationäre Behandlung in einem Krankenhaus oder in einer Rehabilitationseinrichtung wird erbracht, wenn die Aufnahme erforderlich ist, weil das Behandlungsziel anders nicht erreicht werden kann. Sie wird voll- oder teilstationär erbracht. Sie umfaßt im Rahmen des Versorgungsauftrags des Krankenhauses oder der Rehabilitationseinrichtung alle Leistungen, die im Einzelfall für die medizinische Versorgung der Versicherten notwendig sind, insbesondere ärztliche Behandlung, Krankenpflege, Versorgung mit Arznei-, Verband-, Heil- und Hilfsmitteln, Unterkunft und Verpflegung.

(2) Krankenhäuser und Rehabilitationseinrichtungen im Sinne des Absatzes 1 sind die Einrichtungen nach $\$ 107$ des Fünften Buches.

(3) Bei Gesundheitsschäden, für die wegen ihrer Art oder Schwere besondere unfallmedizinische stationäre Behandlung angezeigt ist, wird diese in besonderen Einrich-
tungen erbracht.

\section{Durchführung der Heilbehandlung}

\$ 34. (1) Die Unfallversicherungsträger haben alle Maßnahmen zu treffen, durch die eine möglichst frühzeitig nach dem Versicherungsfall einsetzende und sachgemäß Heilbehandlung und, soweit erforderlich, besondere unfallmedizinische oder Berufskrankheiten-Behandlung gewährleistet wird. Sie können zu diesem Zweck die von den Ärzten und Krankenhäusern zu erfüllenden Voraussetzungen im Hinblick auf die fachliche Befähigung, die sächliche und personelle Ausstattung sowie die zu übernehmenden pflichten festlegen. Sie können daneben nach Art und Schwere des Gesundheitsschadens besondere Verfahren für die Heilbehandlung vorsehen.

(2) Die Unfallversicherungsträger haben an der Durchführung der besonderen unfallmedizinischen Behandlung die Ärzte und Krankenhäuser zu beteiligen, die den nach Absatz 1 Satz 2 festgelegten Anforderungen entsprechen.

(3) Die Verbände der Unfallversicherungsträger sowie die Kassenärztliche Bundesvereinigung und die Kassenzahnärztliche Bundesvereinigung (Kassenärztliche Bundesvereinigungen) schließen unter Berücksichtigung der von den Unfallversicherungsträgern gemäß Absatz 1 Satz 2 und 3 getroffenen Festlegungen mit Wirkung für ihre Mitglieder Verträge über die Durchführung der Heilbehandlung, die Vergütung der Ärzte und Zahnärzte sowie die Art und Weise der Abrechnung. Dem Bundesbeauftragten für den Datenschutz ist rechtzeitig vor Abschluß Gelegenheit zur Stellungnahme zu geben, sofern in den Verträgen die Erhebung, Verarbeitung oder Nutzung von personenbezogenen Daten geregelt werden sollen.

$$
\begin{aligned}
& \text { (4) } \ldots \\
& \text { (5) } \ldots \\
& \text { (6) } \ldots \\
& \text { (7) } \ldots
\end{aligned}
$$

\section{Sozialgesetzbuch (SGB) EIftes Buch (XI) Gesetzliche Pflegeversicherung}

Vorrang von Prävention und medizinischer Rehabilitation

5 5. (1) Die Pflegekassen wirken bei den zuständigen Leistungsträgern darauf hin, daß frühzeitig alle geeigneten Leistungen der Prävention, der Krankenbehandlung und zur medizinischen Rehabilitation eingeleitet werden, um den Eintritt von Pflegebedürftigkeit zu vermeiden.

(2) Die Leistungsträger haben im Rahmen ihres Leistungsrechts auch nach Eintritt der Pflegebedürftigkeit ihre Leistungen zur medizinischen Rehabilitation und ergänzenden Leistungen in vollem Umfang einzusetzen und darauf hinzuwirken, die Pflegebedürftigkeit zu überwinden, zu mindern sowie eine Verschlimmerung zu verhindern.

\section{Vorläufige Leistungen zur medizinischen Rehabilitation}

5 32. (1) Die Pflegekasse erbringt vorläufige Leistungen zur medizinischen Rehabilitation, wenn eine sofortige Leistungserbringung erforderlich ist, um eine unmittelbar drohende Pflegebedürftigkeit zu vermeiden, eine bestehende Pflegebedürftigkeit zu überwinden, zu mindern oder eine Verschlimmerung der Pflegebedürftigkeit zu verhüten, und sonst die sofortige Einleitung der Leistungen gefährdet wäre.

(2) Die Pflegekasse hat zuvor den zuständigen Träger zu unterrichten und auf die Eilbedürftigkeit der Leistungsgewährung hinzuweisen; wird dieser nicht rechtzeitig, spätestens jedoch vier Wochen nach Antragstellung, tätig, erbringt die Pflegekasse die Leistungen vorläufig.

\section{Sozialgesetzbuch (SGB) Neuntes Buch (IX)}

Rehabilitation und Teilhabe behinderter Menschen

Selbstbestimmung und Teilhabe am Leben in der Gesellschaft

5 1. Behinderte oder von Behinderung bedrohte Menschen erhalten Leistungen nach diesem Buch und den für die Rehabilitationsträger geltenden Leistungsgesetzen, um 
ihre Selbstbestimmung und gleichberechtigte Teilhabe am Leben in der Gesellschaft zu fördern, Benachteiligungen zu vermeiden oder ihnen entgegenzuwirken. Dabei wird den besonderen Bedürfnissen behinderter und von Behinderung bedrohter Frauen und Kinder Rechnung getragen.

\section{Behinderung}

\$ 2. (1) Menschen sind behindert, wenn ihre körperliche Funktion, geistige Fähigkeit oder seelische Gesundheit mit hoher Wahrscheinlichkeit länger als sechs Monate von dem für das Lebensalter typischen Zustand abweichen und daher ihre Teilhabe am Leben in der Gesellschaft beeinträchtigt ist. Sie sind von Behinderung bedroht, wenn die Beeinträchtigung zu erwarten ist.

(2) Menschen sind im Sinne des Teils 2 schwerbehindert, wenn bei ihnen ein Grad der Behinderung von wenigstens 50 vorliegt und sie ihren Wohnsitz, ihren gewöhnlichen Aufenthalt oder ihre Beschäftigung auf einem Arbeitsplatz im Sinne des $\$ 73$ rechtmäßig im Geltungsbereich dieses Gesetzbuches haben.

(3) Schwerbehinderten Menschen gleichgestellt werden sollen behinderte Menschen mit einem Grad der Behinderung von weniger als 50, aber wenigstens 30, bei denen die übrigen Voraussetzungen des Absatzes 2 vorliegen, wenn sie infolge ihrer Behinderung ohne die Gleichstellung einen geeigneten Arbeitsplatz im Sinne des $\$ 73$ nicht erlangen oder nicht behalten können (gleichgestellte behinderte Menschen)

\section{Vorrang von Prävention}

5 3. Die Rehabilitationsträger wirken darauf hin, dass der Eintritt einer Behinderung einschließlich einer chronischen Krankheit vermieden wird.

\section{Leistungen zur Teilhabe}

5 4. (1) Die Leistungen zur Teilhabe umfassen die notwendigen Sozialleistungen, um unabhängig von der Ursache der Behinderung

1. die Behinderung abzuwenden, zu beseitigen, zu mindern, ihre Verschlimmerung zu verhüten oder ihre Folgen zu mildern,

. Einschränkungen der Erwerbsfähigkeit oder Pflegebedürftigkeit zu vermeiden, zu überwinden, zu mindern oder eine Verschlimmerung zu verhüten sowie den vorzeitigen Bezug anderer Sozialleistungen zu vermeiden oder laufende Sozialleistungen zu mindern,

3. die Teilhabe am Arbeitsleben entsprechend den Neigungen und Fähigkeiten dauerhaft zu sichern oder

4. die persönliche Entwicklung ganzheitlich zu fördern und die Teilhabe am Leben in der Gesellschaft sowie eine möglichst selbständige und selbstbestimmte Lebensführung zu ermöglichen oder zu erleichtern.

(2) Die Leistungen zur Teilhabe werden zur Erreichung der in Absatz 1 genannten Ziele nach Maßgabe dieses Buches und der für die zuständigen Leistungsträger geltenden besonderen Vorschriften neben anderen Sozialleistungen erbracht. Die Leistungsträger erbringen die Leistungen im Rahmen der für sie geltenden Rechtsvorschriften nach Lage des Einzelfalls so vollständig, umfassend und in gleicher Qualität, dass Leistungen eines anderen Trägers möglichst nicht erforderlich werden.

(3) Leistungen für behinderte oder von Behinderung bedrohte Kinder werden so geplant und gestaltet, dass nach Möglichkeit Kinder nicht von ihrem sozialen Umfeld getrennt und gemeinsam mit nicht behinderten Kindern betreut werden können. Dabei werden behinderte Kinder alters- und entwicklungsentsprechend an der Planung und
Ausgestaltung der einzelnen Hilfen beteiligt und ihre Sorgeberechtigten intensiv in Planung und Gestaltung der Hilfen einbezogen.

\section{Leistungsgruppen}

\$ 5. Zur Teilhabe werden erbracht

1. Leistungen zur medizinischen Rehabilitation,

2. Leistungen zur Teilhabe am Arbeitsleben,

3. unterhaltssichernde und andere ergänzende Leistungen,

3. Leistungen zur Teilhabe am Leben in der Gemeinschaft

\section{Rehabilitationsträger}

6. (1) Träger der Leistungen zur Teilhabe (Rehabilitationsträger) können sein

1. die gesetzlichen Krankenkassen für Leistungen nach $\$ 5 \mathrm{Nr}$. I und 3 ,

2. die Bundesagentur für Arbeit für Leistungen nach $\$ 5 \mathrm{Nr}$. 2 und 3 ,

3. die Träger der gesetzlichen Unfallversicherung für Leistungen nach $\$ 5 \mathrm{Nr}$. 1 bis 4 ,

4. die Träger der gesetzlichen Rentenversicherung für Leistungen nach $\$ 5 \mathrm{Nr}$. 1 bis

, die Träger der Alterssicherung der Landwirte für Leistungen nach $\$ 5 \mathrm{Nr}$. 1 und 3 ,

5. die Träger der Kriegsopferversorgung und die Träger der Kriegsopferfürsorge im Rahmen des Rechts der sozialen Entschädigung bei Gesundheitsschäden für Leistungen nach $\$ 5 \mathrm{Nr}$. 1 bis 4

6. die Träger der öffentlichen Jugendhilfe für Leistungen nach $\$ 5 \mathrm{Nr} .1,2$ und 4 ,

7. die Träger der Sozialhilfe für Leistungen nach $\$ 5 \mathrm{Nr} .1,2$ und 4 .

(2) Die Rehabilitationsträger nehmen ihre Aufgaben selbständig und eigenverantwortlich wahr.

\section{Vorbehalt abweichender Regelungen}

5 7. Die Vorschriften dieses Buches gelten für die Leistungen zur Teilhabe, soweit (neistungsgesetzen nichts Abweichendes ergibt. Die Zuständigkeit und die Voraussetzungen für die Leistungen zu Teilhabe richten sich nach den für den jeweiligen Rehabilitationsträger geltenden Leistungsgesetzen.

\section{Vorrang von Leistungen zur Teilhabe}

\$ 8. (1) Werden bei einem Rehabilitationsträger Sozialleistungen wegen oder unter Berücksichtigung einer Behinderung oder einer drohenden Behinderung beantragt ode erbracht, prüft dieser unabhängig von der Entscheidung über diese Leistungen, ob Leistungen zur Teilhabe voraussichtlich erfolgreich sind.

(2) Leistungen zur Teilhabe haben Vorrang vor Rentenleistungen, die bei erfolgreichen Leistungen zur Teilhabe nicht oder voraussichtlich erst zu einem späteren Zeit-
punkt zu erbringen wären. Dies gilt während des Bezuges einer Rente entsprechend.

(3) Absatz 1 ist auch anzuwenden, um durch Leistungen zur Teilhabe Pflegebedürftigkeit zu vermeiden, zu überwinden, zu mindern oder eine Verschlimmerung zu verhüten.

\section{Wunsch- und Wahlrecht der Leistungsberechtigten}

§ 9. (1) Bei der Entscheidung über die Leistungen und bei der Ausführung der Leistungen zur Teilhabe wird berechtigten Wünschen der Leistungsberechtigten ent sprochen. Dabei wird auch auf die persönliche Lebenssituation, das Alter, das Geschlecht, die Familie sowie die religiösen und weltanschaulichen Bedürfnisse der Leis- 
tungsberechtigten Rücksicht genommen; im Übrigen gilt $\$ 33$ des Ersten Buches. Den besonderen Bedürfnissen behinderter Mütter und Väter bei der Erfüllung ihres Erziehungsauftrages sowie den besonderen Bedürfnissen behinderter Kinder wird Rechnung getragen.

(2) Sachleistungen zur Teilhabe, die nicht in Rehabilitationseinrichtungen auszuführen sind, können auf Antrag der Leistungsberechtigten als Geldleistungen erbracht werden, wenn die Leistungen hierdurch voraussichtlich bei gleicher Wirksamkeit wirtschaftlich zumindest gleichwertig ausgeführt werden können. Für die Beurteilung der Wirksamkeit stellen die Leistungsberechtigten dem Rehabilitationsträger geeignete Unterlagen zur Verfügung. Der Rehabilitationsträger begründet durch Bescheid, wenn er den Wünschen des Leistungsberechtigten nach den Absätzen 1 und 2 nicht entspricht.

(3) Leistungen, Dienste und Einrichtungen lassen den Leistungsberechtigten möglichst viel Raum zu eigenverantwortlicher Gestaltung ihrer Lebensumstände und fördern ihre Selbstbestimmung.

(4) Die Leistungen zur Teilhabe bedürfen der Zustimmung der Leistungsberech-

\section{Koordinierung der Leistungen}

\$ 10. (1) Soweit Leistungen verschiedener Leistungsgruppen oder mehrerer Rehabilitationsträger erforderlich sind, ist der nach $\$ 14$ leistende Rehabilitationsträger dafür verantwortlich, dass die beteiligten Rehabilitationsträger im Benehmen miteinander und in Abstimmung mit den Leistungsberechtigten die nach dem individuellen Bedarf voraussichtlich erforderlichen Leistungen funktionsbezogen feststellen und schriftlich so zusammenstellen, dass sie nahtlos ineinander greifen. Die Leistungen werden entsprechend dem Verlauf der Rehabilitation angepasst und darauf ausgerichtet, den Leistungsberechtigten unter Berücksichtigung der Besonderheiten des Einzelfalls die den Zielen der $\$ \$ 1$ und 4 Abs. 1 entsprechende umfassende Teilhabe am Leben in der Gesellschaft zügig, wirksam, wirtschaftlich und auf Dauer zu ermöglichen. Dabei sichern die Rehabilitationsträger durchgehend das Verfahren entsprechend dem jeweiligen Bedarf und gewährleisten, dass die wirksame und wirtschaftliche Ausführung der Leistungen nach gleichen Maßstäben und Grundsätzen erfolgt.

(2) Absatz 1 gilt entsprechend auch für die Integrationsämter in Bezug auf Leistungen und sonstige Hilfen für schwerbehinderte Menschen nach Teil 2

(3) Den besonderen Bedürfnissen seelisch behinderter oder von einer solchen Behinderung bedrohter Menschen wird Rechnung getragen.

(4) Die datenschutzrechtlichen Regelungen dieses Gesetzbuchs bleiben unberührt.

\section{Zusammenwirken der Leistungen}

$\S$ 11. (1) Soweit es im Einzelfall geboten ist, prüft der zuständige Rehabilitationsträger gleichzeitig mit der Einleitung einer Leistung zur medizinischen Rehabilitation, während ihrer Ausführung und nach ihrem Abschluss, ob durch geeignete Leistungen zur Teilhabe am Arbeitsleben die Erwerbsfähigkeit des behinderten oder von Behinderun bedrohten Menschen erhalten, gebessert oder wiederhergestellt werden $\mathrm{kann}$. Er beteiligt die Bundesagentur für Arbeit nach $\$ 38$.

(2) Wird während einer Leistung zur medizinischen Rehabilitation erkennbar, dass der bisherige Arbeitsplatz gefährdet ist, wird mit den Betroffenen sowie dem zuständigen Rehabilitationsträger unverzüglich geklärt, ob Leistungen zur Teilhabe am Arbeitsleben erforderlich sind.
(3) Bei der Prüfung nach den Absätzen 1 und 2 wird zur Klärung eines Hilfebedarfs nach Teil 2 auch das Integrationsamt beteiligt.

\section{Zusammenarbeit der Rehabilitationsträger}

5 12. (1) Im Rahmen der durch Gesetz, Rechtsverordnung oder allgemeine Verwaltungsvorschrift getroffenen Regelungen sind die Rehabilitationsträger verantwortlich, dass

1. die im Einzelfall erforderlichen Leistungen zur Teilhabe nahtlos, zügig sowie nach Gegenstand, Umfang und Ausführung einheitlich erbracht werden,

2. Abgrenzungsfragen einvernehmlich geklärt werden,

3. Beratung entsprechend den in $\$ \$ 1$ und 4 genannten Zielen geleistet wird,

4. Begutachtungen möglichst nach einheitlichen Grundsätzen durchgeführt werden sowie

5. Prävention entsprechend dem in $\$ 3$ genannten Ziel geleistet wird.

(2) Die Rehabilitationsträger und ihre Verbände sollen zur gemeinsamen Wahrnehmung von Aufgaben zur Teilhabe behinderter Menschen insbesondere regionale Arbeitsgemeinschaften bilden. $\$ 88$ Abs. 1 Satz 1 und Abs. 2 des Zehnten Buches gilt entsprechend.

\section{Gemeinsame Empfehlungen}

5 13. (1) Die Rehabilitationsträger nach $\$ 6$ Abs. $1 \mathrm{Nr}$. 1 bis 5 vereinbaren zur Sicherung der Zusammenarbeit nach $\$ 12$ Abs. 1 gemeinsame Empfehlungen.

(2) Die Rehabilitationsträger nach $\$ 6$ Abs. $1 \mathrm{Nr}$. 1 bis 5 vereinbaren darüber hinaus gemeinsame Empfehlungen,

1. welche Maßnahmen nach $\$ 3$ geeignet sind, um den Eintritt einer Behinderung zu vermeiden, sowie über die statistische Erfassung der Anzahl, des Umfangs und der Wirkungen dieser Maßnahmen,

2. in welchen Fällen und in welcher Weise rehabilitationsbedürftigen Menschen notwendige Leistungen zur Teilhabe angeboten werden, insbesondere um eine durch eine Chronifizierung von Erkrankungen bedingte Behinderung zu verhindern,

3. in welchen Fällen und in welcher Weise die Klärung der im Einzelfall anzustrebenden Ziele und des Bedarfs an Leistungen schriftlich festzuhalten ist sowie über die Ausgestaltung des in $\$ 14$ bestimmten Verfahrens,

4. in welcher Weise die Bundesagentur für Arbeit von den übrigen Rehabilitationsträgern nach $\$ 38$ zu beteiligen ist,

5. wie Leistungen zur Teilhabe zwischen verschiedenen Trägern koordiniert werden

6. in welcher Weise und in welchem Umfang Selbsthilfegruppen, -organisationen und -kontaktstellen, die sich die Prävention, Rehabilitation, Früherkennung und Bewältigung von Krankheiten und Behinderungen zum Ziel gesetzt haben, gefördert werden,

7. wie während der Ausführung ambulanter Leistungen zur Teilhabe Leistungen zum Lebensunterhalt $(\$ 45)$ untereinander und von anderen Entgeltersatzleistungen abzugrenzen sind, soweit für diesen Zeitraum Anspruch auf mehrere Entgeltersatzleistungen besteht,

8. in welchen Fällen und in welcher Weise der behandelnde Hausarzt oder Facharzt und der Betriebs- oder Werksarzt in die Einleitung und Ausführung von Leistungen zur Teilhabe einzubinden sind,

9. zu einem Informationsaustausch mit behinderten Beschäftigten, Arbeitgebern und den in $\$ 83$ genannten Vertretungen zur möglichst frühzeitigen Erkennung des individuellen Bedarfs voraussichtlich erforderlicher Leistungen zur Teilhabe sowie 
10. über ihre Zusammenarbeit mit Sozialdiensten und vergleichbaren Stellen.

(3) Bestehen für einen Rehabilitationsträger Rahmenempfehlungen auf Grund ge-

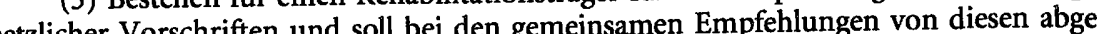
setzlicher Vorschriften und soll bei den gens wichen werden oder sollen die gemeinsamen Empfehlungen Gegenstände betreffen, die nach den gesetzlichen Vorschriften Gegenstand solcher Rahmenempfehlungen werden sollen, stellt der Rehabilitationsträger das Einvernehmen mit den jeweiligen Partnern der Rahmenempfehlungen sicher.

(4) Die Träger der Renten-, Kranken- und Unfallversicherung sowie der Alterssicherung der Landwirte können sich bei der Vereinbarung der gemeinsamen Empfehlungen durch ihre Spitzenverbände vertreten lassen.

(5) An der Vorbereitung der gemeinsamen Empfehlungen werden die Träger der Sozialhilfe und der öffentlichen Jugendhilfe über die Bundesvereinigung der Kommunalen Spitzenverbände, die Bundesarbeitsgemeinschaft der überörtlichen Träger der Sozialhilfe, die Bunerounter in die Bunderte Menschen nach dem Teil Bezug auf Leistungen und sonstige Hilfen fur schwerbenind 2 über die Bundesarbeitsgemeinschaft der Integrationsämter und Hauptfürsorgestellen, beteiligt. Die Träger der Sozialhilfe und der öffentlichen Jugendhilfe orientieren sich bei der Wahrnehmung ihrer Aufgaben nach diesem Buch an den vereinbarten Empfehlungen oder können diesen beitreten.

(6) Die Verbände behinderter Menschen einschließlich der Verbände der Freien 作 Frauen sowie die für die Wahrnehmung der Interessen der ambulanten und stationären Rehabilitationseinrichtungen auf Bundesebene maßgeblichen Spitzenverbände werden an der Vorbereitung der gemeinsamen Empfehlungen beteiligt. Ihren Anliegen wird bei der Ausgestaltung der Empfehlungen nach Möglichkeit Rechnung getragen. Die Empfehder Auster oder von Behindelungen berücksichtigen auch die ber.
rung bedrohter Frauen und Kinder.

(7) Die beteiligten Rehabilitationsträger vereinbaren die gemeinsamen Empfehungen im Rahmen der Bundesarbeitsgemeinschaft für Rehabilitation im Benehmen mit dem Bundesministerium für Arbeit und Soziales und den Ländern auf der Grundlage eines von ihnen innerhalb der Bundesarbeitsgemeinschaft vorbereiteten Vorschlags. Der Bus Bundes und Soziales zu Arbeit und Sozloles zu einem Vorschlag aufgefordert für Rehabilitation den Vorschlag innerhalb von sechs Monaten gefolgt, wenn ihm berechtigte Interessen eines Rehabilitationsträgers nicht entgegenstehen. Einwände nach Satz 4 sind innerhalb von vier Wochen nach Vorlage des Vorschlags auszuräumen.

(8) Die Rehabilitationsträger teilen der Bundesarbeitsgemeinschaft für Rehabilitation jährlich ihre Erfahrungen mit den gemeinsamen Empfehlungen mit, die Träger der Renten-, Kranken- und Unfallversicherung sowie der Alterssicherung der Landwirte über ihre Spitzenverbände. Die Bundesarbeitsgemeinschaft für Rehabilitation stellt dem Bundesministerium für Arbeit und Soziales und den Ländern eine Zusammenfassung zur Verfügung.

(9) Die gemeinsamen Empfehlungen können durch die regional zuständigen Rehabilitationsträger konkretisiert werden.

\section{Zuständigkeitsklärung}

5 14. (1) Werden Leistungen zur Teilhabe beantragt, stellt der Rehabilitationsträger nnerhalb von zwei Wochen nach Eingang des Antrages bei ihm fest, ob er nach dem für ihn geltenden Leistungsgesetz für die Leistung zuständig ist; bei den Krankenkassen um- fasst die Prüfung auch die Leistungspflicht nach $\$ 40$ Abs. 4 des Fünften Buches. Stellt er bei der Prüfung fest, dass er für die Leistung nicht zuständig ist, leitet er den Antrag unverzüglich dem nach seiner Auffassung zuständigen Rehabilitationsträger zu. Muss für eine solche Feststellung die Ursache der Behinderung geklärt werden und ist diese Klärung in der Frist nach Satz 1 nicht möglich, wird der Antrag unverzüglich dem Rehabilitationsträder Frist nach Satz 1 nicht möglich, wird der Antrag unverzüglich dem Rehabilitationstrãger zugeleitet, der die Leistung ohne Rücksicht auf die Ursache erbringt. Wird der Antrag
bei der Bundesagentur für Arbeit gestellt, werden bei der Prüfung nach den Sätzen 1 und 2 Feststellungen nach $\$ 11$ Abs. $2 \mathrm{a} \mathrm{Nr}$. 1 des Sechsten Buches und $\$ 22$ Abs. 2 des Dritten Buches nicht getroffen.

(2) Wird der Antrag nicht weitergeleitet, stellt der Rehabilitationsträger den Rehabilitationsbedarf unverzüglich fest. Muss für diese Feststellung ein Gutachten nicht eingeholt werden, entscheidet der Rehabilitationsträger innerhalb von drei Wochen nach Antragseingang. Wird der Antrag weitergeleitet, gelten die Sätze 1 und 2 für den Rehabilitationsträger, an den der Antrag weitergeleitet worden ist, entsprechend; die in Satz 2 genannte Frist beginnt mit dem Eingang bei diesem Rehabilitationsträger. Ist für die Feststellung des Rehabilitationsbedarfs ein Gutachten erforderlich, wird die Entscheidung innerhalb von zwei Wochen nach Vorliegen des Gutachtens getroffen. Kann der Rehabilitationsträger, an den der Antrag weitergeleitet worden ist, für die beantragte Leistung nicht Rehabilitationsträger nach $\$ 6$ Abs. 1 sein, klärt er unverzüglich mit dem nach seiner Auffassung zuständigen Rehabilitationsträger, von wem und in welcher Weise über den Antrag innerhalb der Fristen nach den Sätzen 2 und 4 entschieden wird und unterrichtet hierüber den Antragsteller.

(3) Die Absätze 1 und 2 gelten sinngemäß, wenn der Rehabilitationsträger Leistungen von Amts wegen erbringt. Dabei tritt an die Stelle des Tages der Antragstellung der Tag der Kenntnis des voraussichtlichen Rehabilitationsbedarfs.

(4) Wird nach Bewilligung der Leistung durch einen Rehabilitationsträger nach Absatz 1 Satz 2 bis 4 festgestellt, dass ein anderer Rehabilitationsträger für die Leistung zuständig ist, erstattet dieser dem Rehabilitationsträger, der die Leistung erbracht hat, dessen Aufwendungen nach den für diesen geltenden Rechtsvorschriften. Die Bundesagentur für Arbeit leitet für die Klärung nach Satz 1 Anträge auf Leistungen zur Teilhabe am Arbeitsleben zur Feststellung nach $\$ 11$ Abs. 2a Nr. 1 des Sechsten Buches an die Träger der Rentenversicherung nur weiter, wenn sie konkrete Anhaltspunkte dafür hat, dass der Träger der Rentenversicherung zur Leistung einer Rente unabhängig von der jeweiligen Arbeitsmarktlage verpflichtet sein könnte. Für unzuständige Rehabilitationsträger, die eine Leistung nach Absatz 2 Satz 1 und 2 erbracht haben, ist $\$ 105$ des Zehnten Buches nicht anzuwenden, es sei denn, die Rehabilitationsträger vereinbaren Abweichendes.

(5) Der Rehabilitationsträger stellt sicher, dass er Sachverständige beauftragen kann, bei denen Zugangs- und Kommunikationsbarrieren nicht bestehen. Ist für die Feststellun des Rehabilitationsbedarfs ein Gutachten erforderlich, beauftragt der Rehabilitationsträge unverzüglich einen geeigneten Sachverständigen. Er benennt den Leistungsberechtigten in der Regel drei möglichst wohnortnahe Sachverständige unter Berücksichtigung bestehender sozialmedizinischer Dienste. Haben sich Leistungsberechtigte für einen benannten Sachverständigen entschieden, wird dem Wunsch Rechnung getragen. Der Sachverständige nimmt eine umfassende sozialmedizinische, bei Bedarf auch psychologische Begutachtung vor und erstellt das Gutachten innerhalb von zwei Wochen nach Auftragserteilung. Die in dem Gutachten getroffenen Feststellungen zum Rehabilitationsbedarf werden den Die in dem Gutachten getroffenen Feststellungen zum Rehabilitationsbedarf werden den
Entscheidungen der Rehabilitationsträger zugrunde gelegt. Die gesetzlichen Aufgaben der Gesundheitsämter bleiben unberührt.

(6) Hält der leistende Rehabilitationsträger weitere Leistungen zur Teilhabe für erforderlich und kann er für diese Leistungen nicht Rehabilitationsträger nach $\$ 6$ Abs. 1 
sein, wird Absatz 1 Satz 2 entsprechend angewendet. Die Leistungsberechtigten werden hierüber unterrichtet.

\section{Erstattung selbstbeschaffter Leistungen}

§ 15. (1) Kann über den Antrag auf Leistungen zur Teilhabe nicht innerhalb der in $\$ 14$ Abs. 2 genannten Fristen entschieden werden, teilt der Rehabilitationsträger dies den Leistungsberechtigten unter Darlegung der Gründe rechtzeitig mit. Erfolgt die Mitteilung nicht oder liegt ein zureichender Grund nicht vor, können Leistungsberechtigte dem Rehabilitationsträger eine angemessene Frist setzen und dabei erklären, dass sie sich nach Ablauf der Frist die erforderliche Leistung selbst beschaffen. Beschaffen sich Leistungsberechtigte nach Ablauf der Frist eine erforderliche Leistung selbst, ist der zuständige Rehabilitationsträger unter Beachtung der Grundsätze der Wirtschaftlichkeit und Sparsamkeit zur Erstattung der Aufwendungen verpflichtet. Die Erstattungspflicht besteht auch, wenn der Rehabilitationsträger eine unaufschiebbare Leistung nicht rechtzeitig erbringen kann oder er eine Leistung zu Unrecht abgelehnt hat. Die Sätze 1 bis 3 gelten nicht für die Träger der Sozialhilfe, der öffentlichen Jugendhilfe und der Kriegsopferfürsorge.

(2) Die Rehabilitationsträger erfassen,

1. in wie vielen Fällen die Fristen nach $\$ 14$ nicht eingehalten wurden,

2. in welchem Umfang sich die Verfahrensdauer vom Eingang der Anträge bis zur Entscheidung über die Anträge verringert hat,

3. in wie vielen Fällen eine Kostenerstattung nach Absatz 1 Satz 3 und 4 erfolgt ist.

\section{Verordnungsermächtigung}

\$ 16. Vereinbaren die Rehabilitationsträger nicht innerhalb von sechs Monaten nachdem das Bundesministerium für Arbeit und Soziales sie dazu aufgefordert hat, ge meinsame Empfehlungen nach $\$ 13$ oder ändern sie unzureichend gewordene Empfehlungen nicht innerhalb dieser Frist, kann das Bundesministerium für Arbeit und Soziales Regelungen durch Rechtsverordnung mit Zustimmung des Bundesrates erlassen.

\section{Ausführung von Leistungen, Persönliches Budget}

§ 17. (1) Der zuständige Rehabilitationsträger kann Leistungen zur Teilhabe 1. allein oder gemeinsam mit anderen Leistungsträgern,

2. durch andere Leistungsträger oder

3. unter Inanspruchnahme von geeigneten, insbesondere auch freien und gemeinnützigen oder privaten Rehabilitationsdiensten und -einrichtungen $(\$ 19)$

ausführen. Er bleibt für die Ausführung der Leistungen verantwortlich. Satz 1 gilt insbesondere dann, wenn der Rehabilitationsträger die Leistung dadurch wirksamer oder wirtschaftlicher erbringen kann.

(2) Auf Antrag können Leistungen zur Teilhabe auch durch ein Persönliches Budget ausgeführt werden, um den Leistungsberechtigten in eigener Verantwortung ein mög lichst selbstbestimmtes Leben zu ermöglichen. Bei der Ausführung des Persönlichen Budgets sind nach Maßgabe des individuell festgestellten Bedarfs die Rehabilitationsträger, die Pflegekassen und die Integrationsämter beteiligt. Das Persönliche Budget wird von den beteiligten Leistungsträgern trägerübergreifend als Komplexleistung erbracht. Budgetfähig sind auch die neben den Leistungen nach Satz 1 erforderlichen Leistungen der Krankenkassen und der Pflegekassen, Leistungen der Träger der Unfallversicherung bei Pflegebedürftigkeit sowie Hilfe zur Pflege der Sozialhilfe, die sich auf alltägliche und regelmäßig wiederkehrende Bedarfe beziehen und als Geldleistungen oder durch Gutscheine erbracht werden können. An die Entscheidung ist der Antragsteller für die Dauer von sechs Monaten gebunden.
(3) Persönliche Budgets werden in der Regel als Geldleistung ausgeführt, bei laufenden Leistungen monatlich. In begründeten Fällen sind Gutscheine auszugeben. Persönliche Budgets werden auf der Grundlage der nach $\$ 10$ Abs. 1 getroffenen Feststellungen so bemessen, dass der individuell festgestellte Bedarf gedeckt wird und die erforder-
liche Beratung und Unterstützung erfolgen kann. Dabei soll die Höhe des Persönlichen Budgets die Kosten aller bisher individuell festgestellten, ohne das Persönliche Budget zu erbringenden Leistungen nicht überschreiten.

(4) Enthält das Persönliche Budget Leistungen mehrerer Leistungsträger, erlässt der nach $\$ 14$ zuständige der beteiligten Leistungsträger im Auftrag und im Namen der anderen beteiligten Leistungsträger den Verwaltungsakt und führt das weitere Verfahren durch. Ein anderer der beteiligten Leistungsträger kann mit den Aufgaben nach Satz 1 beauftragt werden, wenn die beteiligten Leistungsträger dies in Abstimmung mit den Leistungsberechtigten vereinbaren; in diesem Fall gilt $\$ 93$ des Zehnten Buches entsprechend. Die für den handelnden Leistungsträger zuständige Widerspruchsstelle erlässt auch den Widerspruchsbescheid.

(5) ... Modellvorhaben zur Erprobung der Einführung Persönlicher Budgets ...

(6) In der Zeit vom 1. Juli 2004 bis zum 31. Dezember 2007 werden Persönliche Budgets erprobt. ...

\section{Leistungsort}

5 18. Sachleistungen können auch im Ausland erbracht werden, wenn sie dort bei umindest gleicher Qualität und Wirksamkeit wirtschaftlicher ausgeführt werden können. Leistungen zur Teilhabe am Arbeitsleben können im grenznahen Ausland auch ausgeführt werden, wenn sie für die Aufnahme oder Ausübung einer Beschäftigung oder selbständigen Tätigkeit erforderlich sind.

\section{Rehabilitationsdienste und -einrichtungen}

\$ 19. (1) Die Rehabilitationsträger wirken gemeinsam unter Beteiligung der Bundesregierung und der Landesregierungen darauf hin, dass die fachlich und regional erforderlichen Rehabilitationsdienste und -einrichtungen in ausreichender Zahl und Qualität zur Verfügung stehen. Dabei achten sie darauf, dass für eine ausreichende Zahl solcher Rehabilitationsdienste und -einrichtungen Zugangs- und Kommunikationsbarrieren
nicht bestehen. Die Verbände behinderter Menschen einschließlich der Verbände der nicht bestehen. Die Verbände behinderter Menschen einschließlich der Verbände der
Freien Wohlfahrtspflege, der Selbsthilfegruppen und der Interessenvertretungen behinderter Frauen sowie die für die Wahrnehmung der Interessen der ambulanten und stationären Rehabilitationseinrichtungen auf Bundesebene maßgeblichen Spitzenverbände werden beteiligt.

(2) Soweit die Ziele nach Prüfung des Einzelfalls mit vergleichbarer Wirksamkeit erreichbar sind, werden Leistungen unter Berücksichtigung der persönlichen Umstände in ambulanter, teilstationärer oder betrieblicher Form und gegebenenfalls unter Einbeziehung familienentlastender und -unterstützender Dienste erbracht.

(3) Bei Leistungen an behinderte oder von einer Behinderung bedrohte Kinder wird eine gemeinsame Betreuung behinderter und nichtbehinderter Kinder angestrebt.

(4) Nehmen Rehabilitationsträger zur Ausführung von Leistungen besondere Dienste (Rehabilitationsdienste) oder Einrichtungen (Rehabilitationseinrichtungen) in Anspruch, erfolgt die Auswahl danach, welcher Dienst oder welche Einrichtung die Leistung in der am besten geeigneten Form ausführt; .

(5) Rehabilitationsträger können nach den für sie geltenden Rechtsvorschriften Rehabilitationsdienste oder -einrichtungen fördern, wenn dies zweckmäßig ist und die Arbeit dieser Dienste oder Einrichtungen in anderer Weise nicht sichergestellt werden kann. 
(6) Rehabilitationsdienste und -einrichtungen mit gleicher Aufgabenstellung sollen Arbeitsgemeinschaften bilden.

\section{Qualitätssicherung}

$\$$ 20. (1) Die Rehabilitationsträger nach $\$ 6$ Abs. 1 Nr. 1 bis 5 vereinbaren gemeinsame Empfehlungen zur Sicherung und Weiterentwicklung der Qualität der Leistungen, insbesondere zur barrierefreien Leistungserbringung, sowie für die Durchführung vergleichender Qualitätsanalysen als Grundlage für ein effektives Qualitätsmanagement der Leistungserbringer...

(2) Die Erbringer von Leistungen stellen ein Qualitätsmanagement sicher, ...

(2a) ... grundsätzliche Anforderungen an ein einrichtungsinternes Qualitätsmanagement ... sowie ein einheitliches, unabhängiges Zertifizierungsverfahren ...

(3) Die Bundesarbeitsgemeinschaft für Rehabilitation bereitet die Empfehlungen nach Absatz 1 vor. Sie beteiligt die Verbände behinderter Menschen einschließlich der Verbände der Freien Wohlfahrtspflege, der Selbsthilfegruppen und der Interessenvertretungen behinderter Frauen sowie die nach $\$ 19$ Abs. 6 gebildeten Arbeitsgemeinschaften und die für die Wahrnehmung der Interessen der ambulanten und stationären Rehabilitationseinrichtungen auf Bundesebene maßgeblichen Spitzenverbände. Deren Anliegen wird bei der Ausgestaltung der Empfehlungen nach Möglichkeit Rechnung getragen.

(4) $\$ 13$ Abs. 3 ist entsprechend anzuwenden für Vereinbarungen auf Grund gesetzlicher Vorschriften für die Rehabilitationsträger.

\section{Verträge mit Leistungserbringern}

5 21. (1) Die Verträge über die Ausführung von Leistungen durch Rehabilitationsdienste und -einrichtungen, die nicht in der Trägerschaft eines Rehabilitationsträgers stehen, enthalten insbesondere Regelungen über

1. Qualitätsanforderungen an die Ausführung der Leistungen, das beteiligte Personal und die begleitenden Fachdienste,

2. Übernahme von Grundsätzen der Rehabilitationsträger zur Vereinbarung von Vergütungen,

3. Rechte und Pflichten der Teilnehmer, soweit sich diese nicht bereits aus dem Rechtsverhältnis ergeben, das zwischen ihnen und dem Rehabilitationsträger besteht,

4. angemessene Mitwirkungsmöglichkeiten der Teilnehmer an der Ausführung der Leistungen,

5. Geheimhaltung personenbezogener Daten sowie

6. die Beschäftigung eines angemessenen Anteils behinderter, insbesondere schwerbehinderter Frauen.

(2) Die Rehabilitationsträger wirken darauf hin, dass die Verträge nach einheitlichen Grundsätzen abgeschlossen werden; sie können über den Inhalt der Verträge gemeinsame Empfehlungen nach $\$ 13$ sowie Rahmenverträge mit den Arbeitsgemeinschaften der Rehabilitationsdienste und -einrichtungen vereinbaren. Der Bundesbeauftragte für den Datenschutz wird beteiligt.

(3) Verträge mit fachlich nicht geeigneten Diensten oder Einrichtungen werden gekündigt. Stationäre Rehabilitationseinrichtungen sind nur dann als geeignet anzusehen, wenn sie nach $\$ 20$ Abs. 2 Satz 2 zertifiziert sind.

(4) Absatz 1 Nr. 1 und 3 bis 6 wird für eigene Einrichtungen der Rehabilitationsträger entsprechend angew $\$ 21 \mathrm{a}$ Verordnungsermächtigung

Das Bundesministerium für Arbeit und Soziales wird ermächtigt, durch Rechtsverordnung mit Zustimmung des Bundesrates Näheres zum Inhalt und Ausführung des
Persönlichen Budgets, zum Verfahren sowie zur Zuständigkeit bei Beteiligung mehrerer Leistungsträger zu regeln.

\section{Aufgaben}

522 . (1) Gemeinsame örtliche Servicestellen der Rehabilitationsträger bieten behinderten und von Behinderung bedrohten Menschen, ihren Vertrauenspersonen und Personensorgeberechtigten nach $\$ 60$ Beratung und Unterstützung an. Die Beratung und Unterstützung umfasst insbesondere,

1. über Leistungsvoraussetzungen, Leistungen der Rehabilitationsträger, besondere Hilfen im Arbeitsleben sowie über die Verwaltungsabläufe zu informieren,

2. bei der Klärung des Rehabilitationsbedarfs, bei der Inanspruchnahme von Leistungen zur Teilhabe, bei der Inanspruchnahme eines Persönlichen Budgets und der besonderen Hilfen im Arbeitsleben sowie bei der Erfüllung von Mitwirkungspflichten zu helfen,

3. zu klären, welcher Rehabilitationsträger zuständig ist, auf klare und sachdienliche Anträge hinzuwirken und sie an den zuständigen Rehabilitationsträger weiterzuleiten,

4. bei einem Rehabilitationsbedarf, der voraussichtlich ein Gutachten erfordert, den zuständigen Rehabilitationsträger darüber zu informieren,

5. die Entscheidung des zuständigen Rehabilitationsträgers in Fällen, in denen die Notwendigkeit von Leistungen zur Teilhabe offenkundig ist, so umfassend vorzubereiten, dass dieser unverzüglich entscheiden kann,

6. bis zur Entscheidung oder Leistung des Rehabilitationsträgers den behinderten oder von Behinderung bedrohten Menschen unterstützend zu begleiten,

7. bei den Rehabilitationsträgern auf zeitnahe Entscheidungen und Leistungen hinzuwirken und

8. zwischen mehreren Rehabilitationsträgern und Beteiligten auch während der Leistungserbringung zu koordinieren und zu vermitteln.

Die Beratung umfasst unter Beteiligung der Integrationsämter auch die Klärung eines Hilfebedarfs nach Teil 2 dieses Buches. Die Pflegekassen werden bei drohender oder bestehender Pflegebedürftigkeit an der Beratung und Unterstützung durch die gemeinsamen Servicestellen beteiligt. Verbände behinderter Menschen einschließlich der Verbände der Freien Wohlfahrtspflege, der Selbsthilfegruppen und der Interessenvertretungen behinderter Frauen werden mit Einverständnis der behinderten Menschen an der tungen behinderter

$(2) \$ 14$ des Ersten Buches und $\$ 10$ Abs. 2 und $\$ 11$ Abs. 1 bis 3 und 5 des Zwölften Buches bleiben unberührt. Auskünfte nach $\$ 15$ des Ersten Buches über Leistungen zur Teilhabe erteilen alle Rehabilitationsträger.

\section{Servicestellen}

§ 23. (1) Die Rehabilitationsträger stellen unter Nutzung bestehender Strukturen sicher, dass in allen Landkreisen und kreisfreien Städten gemeinsame Servicestellen bestehen. Gemeinsame Servicestellen können für mehrere kleine Landkreise oder kreisfreie Städte eingerichtet werden, wenn eine ortsnahe Beratung und Unterstützung behinderter und von Behinderung bedrohter Menschen gewährleistet ist. In den Ländern Berlin, Bremen und Hamburg werden die Servicestellen entsprechend dem besonderen Verwaltungsaufbau dieser Länder eingerichtet.

(2) Die zuständigen obersten Landessozialbehörden wirken mit Unterstützung der Spitzenverbände der Rehabilitationsträger darauf hin, dass die gemeinsamen Servicestellen unverzüglich eingerichtet werden. 
(3) Die gemeinsamen Servicestellen werden so ausgestattet, dass sie ihre Aufgaben umfassend und qualifiziert erfüllen können, Zugangs- und Kommunikationsbarrieren nicht bestehen und Wartezeiten in der Regel vermieden werden. Hierfür wird besonders qualifiziertes Personal mit breiten Fachkenntnissen insbesondere des Rehabilitationsrechts und der Praxis eingesetzt. $\$ 112$ Abs. 3 ist sinngemäß anzuwenden.

(4) In den Servicestellen dürfen Sozialdaten nur erhoben, verarbeitet und genutzt werden, soweit dies zur Erfüllung der Aufgaben nach $\$ 22 \mathrm{Abs.} 1$ erforderlich ist.

\section{Bericht}

§ 24. (1) Die Rehabilitationsträger, die Träger der Renten-, Kranken- und Unfallversicherung über ihre Spitzenverbände, teilen der Bundesarbeitsgemeinschaft für Rehabilitation im Abstand von drei Jahren, erstmals im Jahre 2004, ihre Erfahrungen über die Einrichtung der gemeinsamen Servicestellen, die Durchführung und Erfüllung ihrer Aufgaben, die Einhaltung des Datenschutzes und mögliche Verbesserungen mit. Personenbezogene Daten werden anonymisiert.

(2) Die Bundesarbeitsgemeinschaft für Rehabilitation bereitet die Mitteilungen der Rehabilitationsträger auf, beteiligt hierbei die zuständigen obersten Landessozialbehörden, erörtert die Mitteilungen auf Landesebene mit den Verbänden behinderter Menschen einschließlich der Verbände der Freien Wohlfahrtspflege, der Selbsthilfegruppen und der Interessenvertretungen behinderter Frauen und berichtet unverzüglich dem Bundesministerium für Arbeit und Soziales und den Ländern.

\section{Verordnungsermächtigung}

5 25. Sind gemeinsame Servicestellen nach $\$ 23$ Abs. 1 nicht bis zum 31. Dezember 2002 in allen Landkreisen und kreisfreien Städten eingerichtet, bestimmt das Bundesministerium für Arbeit und Soziales durch Rechtsverordnung mit Zustimmung des Bundesrates das Nähere über den Ort der Einrichtung, den Rehabilitationsträger, bei dem die gemeinsame Servicestelle eingerichtet wird und der für die Einrichtung verantwortlich ist, den Zeitpunkt, zu dem die Einrichtung abgeschlossen sein muss, sowie über die Organisation, insbesondere entsprechend ihrem Anteil an den Leistungen zur Teilhabe über Art und Umfang der Beteiligung der Rehabilitationsträger in den gemeinsamen Servicestellen.

\section{Leistungen zur medizinischen Rehabilitation}

\$26. (1) Zur medizinischen Rehabilitation behinderter und von Behinderung bedrohter Menschen werden die erforderlichen Leistungen erbracht, um

1. Behinderungen einschließlich chronischer Krankheiten abzuwenden, zu beseitigen, zu mindern, auszugleichen, eine Verschlimmerung zu verhüten oder

2. Einschränkungen der Erwerbsfähigkeit und Pflegebedürftigkeit zu vermeiden, zu überwinden, zu mindern, eine Verschlimmerung zu verhüten sowie den vorzeitigen Bezug von laufenden Sozialleistungen $\mathrm{zu}$ vermeiden oder laufende Sozialleistungen $\mathrm{zu}$ mindern.

(2) Leistungen zur medizinischen Rehabilitation umfassen insbesondere

1. Behandlung durch Ärzte, Zahnärzte und Angehörige anderer Heilberufe, soweit deren Leistungen unter ärztlicher Aufsicht oder auf ärztliche Anordnung ausgeführt werden, einschließlich der Anleitung, eigene Heilungskräfte zu entwickeln,

2. Früherkennung und Frühförderung behinderter und von Behinderung bedrohter Kinder,

3. Arznei- und Verbandmittel,
4. Heilmittel einschließlich physikalischer, Sprach- und Beschäftigungstherapie,

5. Psychotherapie als ärztliche und psychotherapeutische Behandlung,

6. Hilfsmittel,

7. Belastungserprobung und Arbeitstherapie.

(3) Bestandteil der Leistungen nach Absatz 1 sind auch medizinische, psychologische und pädagogische Hilfen, soweit diese Leistungen im Einzelfall erforderlich sind, um die in Absatz 1 genannten Ziele zu erreichen oder zu sichern und Krankheitsfolgen zu vermeiden, zu überwinden, zu mindern oder ihre Verschlimmerung zu verhüten, insbesondere

1. Hilfen zur Unterstützung bei der Krankheits- und Behinderungsverarbeitung,

2. Aktivierung von Selbsthilfepotentialen,

3. mit Zustimmung der Leistungsberechtigten Information und Beratung von Partnern und Angehörigen sowie von Vorgesetzten und Kollegen,

4. Vermittlung von Kontakten zu örtlichen Selbsthilfe- und Beratungsmöglichkeiten,

5. Hilfen zur seelischen Stabilisierung und zur Förderung der sozialen Kompetenz unter anderem durch Training sozialer und kommunikativer Fähigkeiten und im Umgang mit Krisensituationen,

6. Training lebenspraktischer Fähigkeiten,

7. Anleitung und Motivation zur Inanspruchnahme von Leistungen der medizinischen Rehabilitation.

\section{Krankenbehandlung und Rehabilitation}

$\S$ 27. Die in $\$ 26$ Abs. 1 genannten Ziele sowie $\$ 10$ gelten auch bei Leistungen der Krankenbehandlung.

\section{Stufenweise Wiedereingliederung}

5 28. Können arbeitsunfähige Leistungsberechtigte nach ärztlicher Feststellung ihre bisherige Tätigkeit teilweise verrichten und können sie durch eine stufenweise Wiederaufnahme ihrer Tätigkeit voraussichtlich besser wieder in das Erwerbsleben eingegliedert werden, sollen die medizinischen und die sie ergänzenden Leistungen entsprechend dieser Zielsetzung erbracht werden.

\section{Förderung der Selbsthilfe}

$\$ 29$. Selbsthilfegruppen, -organisationen und -kontaktstellen, die sich die Prävention, Rehabilitation, Früherkennung, Behandlung und Bewältigung von Krankheiten und Behinderungen zum Ziel gesetzt haben, sollen nach einheitlichen Grundsätzen gefördert werden.

\section{Früherkennung und Frühförderung}

$\$ 30$. (1) Die medizinischen Leistungen zur Früherkennung und Frühförderung behinderter und von Behinderung bedrohter Kinder nach $\$ 26$ Abs. 2 Nr. 2 umfassen auch

1. die medizinischen Leistungen der mit dieser Zielsetzung fachübergreifend arbeitenden Dienste und Einrichtungen,

2. nichtärztliche sozialpädiatrische, psychologische, heilpädagogische, psychosoziale Leistungen und die Beratung der Erziehungsberechtigten, auch in fachübergreifend arbeitenden Diensten und Einrichtungen, wenn sie unter ärztlicher Verantwortung erbracht werden und erforderlich sind, um eine drohende oder bereits eingetretene Behinderung 
zum frühestmöglichen Zeitpunkt zu erkennen und einen individuellen Behandlungsplan aufzustellen.

Leistungen nach Satz 1 werden als Komplexleistung in Verbindung mit heilpädagogischen Leistungen $(\$ 56)$ erbracht

(2) Leistungen zur Früherkennung und Frühförderung behinderter und von Behinderung bedrohter Kinder umfassen des Weiteren nichtärztliche therapeutische, psychologische, heilpädagogische, sonderpädagogische, psychosoziale Leistungen und die Beratung der Erziehungsberechtigten durch interdisziplinäre Frühförderstellen, wenn sie erforderlich sind, um eine drohende oder bereits eingetretene Behinderung zum frühestmöglichen Zeitpunkt zu erkennen oder die Behinderung durch gezielte Förder- und Behandlungsmaßnahmen auszugleichen oder zu mildern.

(3) Zur Abgrenzung der in den Absätzen 1 und 2 genannten Leistungen und der sonstigen Leistungen dieser Dienste und Einrichtungen ... werden gemeinsame Empfehlungen vereinbart; ...

\section{Hilfsmittel}

§ 31. (1) Hilfsmittel (Körperersatzstücke sowie orthopädische und andere Hilfsmittel) nach $\$ 26$ Abs. $2 \mathrm{Nr} .6$ umfassen die Hilfen, die von den Leistungsempfängern getragen oder mitgeführt oder bei einem Wohnungswechsel mitgenommen werden können und unter Berücksichtigung der Umstände des Einzelfalles erforderlich sind, um

1. einer drohenden Behinderung vorzubeugen,

2. den Erfolg einer Heilbehandlung zu sichern oder

3. eine Behinderung bei der Befriedigung von Grundbedürfnissen des täglichen Lebens auszugleichen, soweit sie nicht allgemeine Gebrauchsgegenstände des täglichen Lebens sind.

(2) Der Anspruch umfasst auch die notwendige Änderung, Instandhaltung, Ersatzbeschaffung sowie die Ausbildung im Gebrauch der Hilfsmittel. Der Rehabilitationsträger soll

1. vor einer Ersatzbeschaffung prüfen, ob eine Änderung oder Instandsetzung von bisher benutzten Hilfsmitteln wirtschaftlicher und gleich wirksam ist

2. die Bewilligung der Hilfsmittel davon abhängig machen, dass die behinderten Menschen sie sich anpassen oder sich in ihrem Gebrauch ausbilden lassen.

(3) Wählen Leistungsempfänger ein geeignetes Hilfsmittel in einer aufwendigeren Ausführung als notwendig, tragen sie die Mehrkosten selbst.

(4) Hilfsmittel können auch leihweise überlassen werden. In diesem Fall gelten die Absätze 2 und 3 entsprechend.

\section{Verordnungsermächtigungen}

5 32. Das Bundesministerium für Arbeit und Soziales wird ermächtigt, durch Rechtsverordnung mit Zustimmung des Bundesrates

1. Näheres zur Abgrenzung der in $\$ 30 \mathrm{Abs} .1$ und 2 genannten Leistungen und der sonstigen Leistungen dieser Dienste und Einrichtungen, zur Übernahme oder Teilung der Kosten zwischen den beteiligten Rehabilitationsträgern, zur Vereinbarung und Abrechnung der Entgelte sowie zur Finanzierung zu regeln, ...

2. Näheres zur Auswahl der im Einzelfall geeigneten Hilfsmittel, insbesondere zum Verfahren, zur Eignungsprüfung, Dokumentation und leihweisen Überlassung der Hilfsmittel sowie zur Zusammenarbeit der anderen Rehabilitationsträger mit den orthopädischen Versorgungsstellen zu regeln.

\section{Ergänzende Leistungen}

5 44. (1) Die Leistungen zur medizinischen Rehabilitation und zur Teilhabe am Arbeitsleben der in $\$ 6$ Abs. 1 Nr. 1 bis 5 genannten Rehabilitationsträger werden ergänzt durch

1. Krankengeld, Versorgungskrankengeld, Verletztengeld, Übergangsgeld, Ausbildungsgeld oder Unterhaltsbeihilfe,

2. Beiträge und Beitragszuschüsse ...

3. ärztlich verordneten Rehabilitationssport in Gruppen unter ärztlicher Betreuung und Überwachung, einschließlich Übungen für behinderte oder von Behinderung bedrohte Frauen und Mädchen, die der Stärkung des Selbstbewusstseins dienen,

4. ärztlich verordnetes Funktionstraining in Gruppen unter fachkundiger Anleitung und Überwachung,

5. Reisekosten,

6. Betriebs- oder Haushaltshilfe und Kinderbetreuungskosten.

(2) Ist der Schutz behinderter Menschen bei Krankheit oder Pflege während der Teilnahme an Leistungen zur Teilhabe am Arbeitsleben nicht anderweitig sichergestellt, ...

\section{Leistungen zum Lebensunterhalt}

§ 45. (1) Im Zusammenhang mit Leistungen zur medizinischen Rehabilitation leis-

1. die gesetzlichen Krankenkassen Krankengeld ...

2. die Träger der Unfallversicherung Verletztengeld ...,

3. die Träger der Rentenversicherung Übergangsgeld ...,

4. die Träger der Kriegsopferversorgung Versorgungskrankengeld ...

(2) $-(6)$...

(7) Wird bei ambulanter Ausführung von Leistungen zur medizinischen Rehabilitation Verletztengeld, Versorgungskrankengeld oder Übergangsgeld geleistet, kann der Rehabilitationsträger im Rahmen der nach $\$ 13$ Abs. 2 Nr. 7 vereinbarten Empfehlung eine Erstattung seiner Aufwendungen für diese Leistungen verlangen.

(8) ...

\section{Pflichten Personensorgeberechtigter}

§ 60. Eltern, Vormünder, Pfleger und Betreuer, die bei ihrer Personensorge anvertrauten Menschen Behinderungen ( $\$ 2$ Abs. 1) wahrnehmen oder durch die in $\$ 61$ genannten Personen hierauf hingewiesen werden, sollen im Rahmen ihres Erziehungsoder Betreuungsauftrags die behinderten Menschen einer gemeinsamen Servicestelle oder einer sonstigen Beratungsstelle für Rehabilitation oder einem Arzt zur Beratung über die geeigneten Leistungen zur Teilhabe vorstellen.

\section{Sicherung der Beratung behinderter Menschen}

\$ 61. (1) Die Beratung der Ärzte, denen eine Person nach $\$ 60$ vorgestellt wird, erstreckt sich auf die geeigneten Leistungen zur Teilhabe. Dabei weisen sie auf die Möglich keit der Beratung durch eine gemeinsame Servicestelle oder eine sonstige Beratungsstelle für Rehabilitation hin. Bei Menschen, bei denen der Eintritt der Behinderung nach allfür Rehabilitation hin. Bei Menschen, bei denen der Eintritt der Behinderung nach allgemeiner ärztlicher Erkenntnis zu erwarten ist, wird entsprechend verfahren. Werdende gewiesen.

(2) Hebammen, Entbindungspfleger, Medizinalpersonen außer Ärzten, Lehrer, Sozialarbeiter, Jugendleiter und Erzieher, die bei Ausübung ihres Berufs Behinderungen ( $\$ 2$ 
Abs. 1) wahrnehmen, weisen die Personensorgeberechtigten auf die Behinderung und auf die Beratungsangebote nach $\$ 60$ hin.

3) Nehmen Medizinalpersonen außer Ärzten und Sozialarbeiter bei Ausübung ihres Berufs Behinderungen ( $\$ 2$ Abs. 1) bei volljährigen Menschen wahr, empfehlen sie diesen Menschen oder den für sie bestellten Betreuern, eine Beratungsstelle für Rehabilitation oder einen Arzt zur Beratung über die geeigneten Leistungen zur Teilhabe aufzusuchen.

\section{Landesärzte}

5 62. (1) In den Ländern können Landesärzte bestellt werden, die über besondere Efahrungen in der Hilfe für behinderte und von Behinderung bedrohte Menschen verfügen.

2) Die Landesärzte haben vor allem die Aufgabe,

1. Gutachten für die Landesbehörden, die für das Gesundheitswesen und die Sozialhilfe zuständig sind, sowie für die zuständigen Träger der Sozialhilfe in besonders schwierig gelagerten Einzelfällen oder in Fällen von grundsätzlicher Bedeutung zu erstatten,

2. die für das Gesundheitswesen zuständigen obersten Landesbehörden beim Erstellen von Konzeptionen, Situations- und Bedarfsanalysen und bei der Landesplanung zur Teilhabe behinderter und von Behinderung bedrohter Menschen zu beraten und zu unterstützen sowie selbst entsprechende Initiativen zu ergreifen,

3. die für das Gesundheitswesen zuständigen Landesbehörden über Art und Ursachen von Behinderungen und notwendige Hilfen sowie über den Erfolg von Leistungen zur Teilhabe behinderter und von Behinderung bedrohter Menschen regelmäßig zu unterrichten.

\section{Klagerecht der Verbände}

5 63. Werden behinderte Menschen in ihren Rechten nach diesem Buch verletzt, können an ihrer Stelle und mit ihrem Einverständnis Verbände klagen, ..

\section{Beirat für die Teilhabe behinderter Menschen}

\$ 64. (1) Beim Bundesministerium für Arbeit und Soziales wird ein Beirat für die Teilhabe behinderter Menschen gebildet, der es in Fragen der Teilhabe behinderter Menschen berät und bei Aufgaben der Koordinierung unterstützt. Zu den Aufgaben des Beirats gehören insbesondere auch

1. die Unterstützung bei der Förderung von Rehabilitationseinrichtungen und die Mitwirkung bei der Vergabe der Mittel des Ausgleichsfonds,

2. die Anregung und Koordinierung von Maßnahmen zur Evaluierung der in diesem Buch getroffenen Regelungen im Rahmen der Rehabilitationsforschung und als forschungsbegleitender Ausschuss die Unterstützung des Ministeriums bei der Festlegung von Fragestellungen und Kriterien.

Das Bundesministerium für Arbeit und Soziales trifft Entscheidungen über die Vergabe der Mittel des Ausgleichsfonds nur auf Grund von Vorschlägen des Beirats.

(2) Der Beirat besteht aus 48 Mitgliedern....
Reinhard Resch

\section{Medizinische Rehabilitation in Österreich: Leistungs- recht und Berührungspunkte zum Arbeitsrecht}

\section{Einleitung}

Im Folgenden geht es darum, ausgewählte Einzelfragen und Randthemen im Zusammenhang mit der medizinischen Rehabilitation zu untersuchen. Nach allgemeinen Überlegungen zur Qualifikation als Pflichtaufgabe (2.) werden mit der medizinischen Rehabilitation im Zusammenhang stehende SV-Leistungen untersucht (3.). Abschließend werden zusammenhängende Fragen im Arbeitsrecht wie Entgeltfortzahlung und Bestandsschutz des Arbeitsvertrags erörtert (4.).

\section{Anspruch auf medizinische Rehabilitation?} 2.1 Pflichtleistung im überschneidenden Bereich

Es wurde oben bereits ausgeführt, dass nach der gesetzlichen Konzeption gerade kein Anspruch auf medizinische Rehabilitation in der gesetzlichen KV bestehen soll, sondern eine bloße Pflichtaufgabe vorliegt. $\left.{ }^{1}\right)$ Es wurde weiters schon ausgeführt, dass die Abgrenzung zur Krankenbehandlung über weite Strecken verschwimmt bzw es einen Bereich gibt, in dem wir eine echte Überschneidung haben, ein und dieselbe Maßnahme sowohl Krankenbehandlung als auch medizinische Rehabilitation sein kann. ${ }^{2}$ ) Damit stellt sich aber die Frage, wo wir eine Behandlung im überschneidenden Bereich einzuordnen haben: Bei der Krankenbehandlung (auf die ein Rechtsanspruch besteht) oder bei der medizinischen Rehabilitation in der KV (die als Pflichtaufgabe konzipiert ist). Indem der Gesetzgeber mit der ausdrücklichen Regelung der medizinischen Rehabilitation im Rahmen der 50. ASVG-Novelle keine Einschränkung des bisherigen Leistungskatalogs im Fall der Krankheit vor Augen gehabt hat, sondern ${ }^{3}$ ) zusätzliche Leistungen einführen wollte, spricht dies dafür, dass es im überschneidenden Bereich im Zweifel beim Leistungsanspruch bleibt, insoweit gleichzeitig der Versicherungsfall der Krankheit vorliegt, weil durch die Maßnahme ein günstiger Einfluss auf die Krankheit ausgeübt wird und dieselbe Maßnahme auch im Kreis der Pflichtleistungen bei Krankheit enthalten ist. Der 50. ASVG-Novelle ist nich der Wille zu unterstellen, in bereits bestehende Pflichtleistungen der gesetzlichen

1) Vgl die RV zur 50. ASVG-Novelle, 284 BlgNR 18. GP 27; vgl dazu bereits oben Rudda 37 und 50

$\left.{ }^{2}\right)$ Oben Endel 2 ff.

$\left.{ }^{3}\right)$ Wenngleich, wie die Konstruktion als Pflichtaufgabe zeigt, zögernd. 
KV zu Lasten der Versicherten einzugreifen und diese zu bloßen Pflichtaufgaben abzuwerten.

Bloße Pflichtaufgabe gem $\$ 154$ a ASVG verbleiben jene Maßnahmen, die keine Krankenbehandlung sind (weil durch sie kein regelwidriger Körper- oder Geisteszustand positiv beeinflusst wird) und die auch sonst nicht in der abschlieBenden Auflistung der Pflichtleistungen der gesetzlichen KV enthalten sind (Pflichtaufgabe wären etwa Kuraufenthalte, wenn sie eine Maßnahme der medizinischen Rehabilitation darstellen $\left.{ }^{4}\right)$ ). Auf die erforderliche ärztliche Hilfe ( $\$ 154 a$ Abs 2 Z 3 ASVG) wird wohl jedenfalls ein Leistungsanspruch bestehen, insofern geht $\$ 135$ ASVG vor, sofern im Rechtssinn eine Krankenbehandlung vorliegt. ${ }^{5}$ )

Gehen wir noch einen Schritt weiter: Soweit wir uns nun im überschneidenden Bereich von Krankenbehandlung und medizinischer Rehabilitation befinden und formell eine Leistung der medizinischen Rehabilitation auch beansprucht worden ist, kann der Versicherte mit der Leistungsbewilligung die doch ganz erheblichen Vorteile einer medizinischen Rehabilitationsmaßnahme im Leistungsrecht gegenüber einer Krankenbehandlung beanspruchen ${ }^{6}$ ) (keine Obergrenze für Hilfsmittelkosten ${ }^{7}$ ) und keine Rezeptgebühr für Heilmittel $\left.{ }^{8}\right)$ ).

\subsection{Ermessen und bescheidmäßiger Abspruch}

Der heftigste Diskussionspunkt in der Rechtswissenschaft in Zusammenhang mit Fragen der medizinischen Rehabilitation ist die Frage, inwieweit die Entscheidung des SV-Trägers über die medizinische Rehabilitation rechtlich nachprüfbar ist. Die gesetzliche Konzeption einer Pflichtaufgabe legt nahe, dass der Träger zwar zur Leistungserbringung bei Vorliegen der Voraussetzungen einseitig verpflichtet wird, „dieser Verpflichtung aber kein individueller Leistungsanspruch gegenübersteht “. ${ }^{9}$ ) An sich hatte der Gesetzgeber beabsichtigt, ab dem Jahr 1994 die Rehabilitation in der KV in eine Pflichtleistung, auf die ein individueller Rechtsanspruch besteht, umzuwandeln. ${ }^{10}$ ) In weiterer Folge hat er aber die in Aussicht gestellte Rechtsänderung dann doch nicht vorgenommen, was die Vergänglichkeit politischer Versprechen zeigt.

Kletter $^{11}$ ) weist in einer ausführlichen Studie zu Recht darauf hin, dass die Konzeption des Gesetzgebers bei der 50. ASVG-Novelle klar darauf hinausgelaufen ist, dem Versicherten keinen Rechtsschutz einzuräumen. Er schließt daraus,

4) Diese können ja als Maßnahme der medizinischen Rehabilitation gem $\$ 154 \mathrm{a}$ ASVG gewährt werden (so ausdrücklich etwa $\$ 11$ der RL für die Erbringung von Leistungen im Rahmen der Rehabilitation sowie von Leistungen im Rahmen der Festigung der Gesundheit und der Gesundheitsvorsorge - RRK 2005, kundgemacht unter www. avsv.at)

5) Erfordernis eines günstigen Einflusses auf die Krankheit, wobei die Linderung von eiden oder die bloße Lebensverlängerung genügen (vgl etwa Resch, Sozialrecht ${ }^{4} 58$ ).

$\left.{ }^{6}\right)$ Vgl dazu oben Rudda (50).

7) $\$ 154$ Abs 1 ASVG

$\left.{ }^{8}\right) \$ 136$ Abs 3 ASVG

) So ausdrücklich die RV zur 50. ASVG-Novelle 284 BlgNR 18. GP 27.

${ }^{10)}$ RV 284 BlgNR 18. GP 27.

11) In Karl (Hrsg), Jahrbuch Sozialversicherungsrecht 08 (2008) 57 ff, insb 69 ff. dass de lege lata - mag auch die Regelung verfassungswidrig sein - das SV-Recht keine Rechtsgrundlage für einen bescheidmäßigen Abspruch über die Leistung und eine nachfolgende sozialgerichtliche Entscheidung vorsieht.

Der $\mathrm{OGH}^{12}$ ) geht - unter Bezugnahme vor allem auf die Lehrmeinung von Oberndorfer ${ }^{13}$ ) zu den freiwilligen Leistungen - dagegen davon aus, dass eine gerichtliche Überprüfung einer als Pflichtaufgabe gewährten Leistung möglich ist. Es besteht ein Anspruch des Versicherten auf gesetzmäßige Ermessensausübung, die auch verfahrensmäßig nachprüfbar sein muss. Nötig ist eine Rechtskontrolle ob die Behörde bei der Leistungsgewährung das ihr zukommende Ermessen im Sinn des Gesetzes ausgeübt hat. Bei der Ermessensprüfung können auch finanzielle Gesichtspunkte eine Grundlage für einen negativen Ermessensgebrauch sein. Das Gericht soll allerdings nicht befugt sein, eine zulässige Ermessensentscheidung eines SV-Trägers durch eine andere zu ersetzen. ${ }^{14}$ ) Wird im Verfahren vom Träger vorgebracht, dass die freiwillige Leistung mangels finanzieller Bedeckung zu versagen ist, hat der Träger dem Gericht die hierfür maßgeblichen Tatsachengrundlagen offen zu legen, ${ }^{15}$ ) wobei diese Aussage, da auf eine freiwillige Leistung bezogen, gerade nicht auf die Pflichtaufgabe der medizinischen Rehabilitation übertragen werden kann.

Der OGH ist damit den zahlreichen Stimmen in der Lehre zur Überprüfbarkeit von freiwilligen Leistungen gefolg ${ }^{16}$ ) und hat diese Position - konsequenterweise - erst Recht auch auf die Pflichtaufgaben ausgedehnt, wobei er sich insofern der Zustimmung eines Teils der Lehre sicher sein kann. ${ }^{17}$ )

Wo liegt nun der Streitpunkt: Unbestritten ist die Feststellung, dass freiwillige Leistungen und Pflichtaufgaben im Rahmen der Hoheitsverwaltung vom Träger geleistet werden. ${ }^{18}$ ) Daraus folgert nun der OGH, dass nach dem Konzept

${ }^{12}$ ) OGH 18. 2. 2003, 10 ObS 258/02t DRdA 2004/22, 263 (Naderhirn) = ZAS 2004/31, 83 (Haslinger) = SZ 2003/14 = SSV-NF 17/17; 23. 5. 2005, $10 \mathrm{ObS} 7 / 05 \mathrm{k} \mathrm{SZ} \mathrm{2005/80=}$ SSV-NF 19/34 = SVSlg 52.318.

$\left.{ }^{13}\right)$ Grundprobleme des Verwaltungsverfahrens in der österreichischen Sozialversicherung, ZAS 1973, $215 \mathrm{f}$.

$\left.{ }^{14}\right)$ Neumayr in Neumayr/Reissner, Zeller Kommentar zum Arbeitsrecht (2006) $\$ 65$ ASGG Rz 12; Oberndorfer/Muzak in Tomandl (Hrsg) System des österreichischen Sozialversicherungsrechts (Loseblattausgabe ab 1980, Stand 21. Lieferung 2008) 6.1.3.2. (654); ASG Wien 27. 7. 2004, 32 Cgs 63/04w SVSlg 52.333.

$\left.{ }^{15}\right)$ OGH 23. 5. 2005, $10 \mathrm{ObS} 7 / 05 \mathrm{k} \mathrm{SZ} \mathrm{2005/80} \mathrm{=} \mathrm{SSV-NF} \mathrm{19/34} \mathrm{=} \mathrm{SVSlg} \mathrm{52.318.}$

16) Obendorfer/Muzak in Tomandl (Hrsg), aaO 6.1.3.2. (653 f); prägnant etwa Öh linger, ZAS 1977, 226; weiters Schrammel, Verfügungen über Leistungsansprüche aus der Sozialversicherung (1982) 79 f; Bernard, Der Rechtsschutz bei Verweigerung freiwilliger Leistungen aus der Krankenversicherung, ZAS 1992, 114 f; M. Binder, ZAS 1992, 100 Fink, Die sukzessive Zuständigkeit im Verfahren in Sozialrechtssachen (1995) $155 \mathrm{ff} ; \mathrm{Neu}$ mayr in ZellKomm $\$ 65$ ASGG Rz 12; aA dagegen Kuderna, ASGG ${ }^{2}(1996) 429 \mathrm{f}$ ( $\$ 65$ Anm 3); Haslinger, ZAS 2004, 186 ff; Kletter, aaO.

17) Jabornegg/Resch, Rehabilitation vor Rente, ZAS 1999, 72; Resch, Sozialrecht ${ }^{4}$ (2008) 13; Naderhirn, DRdA 2004, 268; Teschner/Widlar/Pöltner, ASVG - Allgemeine Sozialversicherung 856/9 (\$154a Anm 2); Pfersmann, Bemerkenswertes aus der SZ 2005/I, ÖJZ 2007, 933.

$\left.{ }^{18}\right) \mathrm{MwN}$ ausführlich zuletzt Kletter, aaO 65; vgl etwa auch Walter, Staatshaftung aus der Sicht der Sozialversicherungsträger, SozSi 1986, 111; OGH 18. 2. 2003, 10 ObS 258/02t 
der Bundesverfassung die hoheitliche Entscheidung einer rechtsstaatlichen Anforderungen genügenden Nachprüfung unterliegen muss. Auch dies wird in der Lehre im Grundsatz nicht bestritten. Als Rechtsgrundlage im Verfassungsrecht wird Art 130 Abs 2 B-VG angesehen und daraus gefolgert, dass dies entsprechend auch für Leistungssachen zu gelten habe. Erst hier scheiden sich die Geister: Dem OGH genügt diese verfassungsrechtliche Vorgabe, um anhand des nicht eindeutig gegenteiligen Gesetzeswortlautes die sozialgerichtliche Nachprüfbarkeit der behördlichen Ermessensübung zu folgern (methodologisch wären wir damit im Terrain einer verfassungskonformen Interpretation von Gesetzen $\left.{ }^{19}\right)$ ), während Kletter eine Sanierung durch den Gesetzgeber für erforderlich hält, weil der gegenteilige Wille des historischen Gesetzgebers zu eindeutig ist. Kletter sieht in der vom Willen des historischen Gesetzgebers gerade nicht mitgetragenen, vom OGH aber angenommenen sukzessiven Kompetenz bei den freiwilligen Leistun gen und den Pflichtaufgaben eine Verletzung des Rechts auf Selbstverwaltung: Der OGH dürfe ohne ausdrückliche gesetzliche Grundlage in das geschützte Recht auf Selbstverwaltung nicht eingreifen, wobei er sich auf verfassungsgerichtliche Judikatur zur Gemeindeselbstverwaltung bezieht. ${ }^{20}$ ) Der Gang zum VfGH hätte sicher den Vorteil von Rechtssicherheit für die Vollziehung und hätte den Gesetzgeber die Chance gegeben, die Problematik verfassungskonform klar zu regeln.

Ich halte die Lösung des OGH für methodologisch vertretbar. Sie ermöglicht eine interpretative Sanierung einer verfassungsrechtlich problematischen Verwaltungspraxis, mag dieser Praxis auch "nur" der Vorwurf gemacht werden können, dass sie den klaren Willen des historischen Gesetzgebers umsetzt. Angesichts des offenen Wortlauts erscheint insofern eine verfassungskonforme Interpretation noch zulässig. Die klar gegenteiligen Gesetzesmaterialien sind insofern nicht bindend, ist doch der Wille des Gesetzgebers nicht ausreichend kla im Gesetzestext zum Ausdruck gekommen. ${ }^{21}$ ) Die verfassungskonforme Interpretation darf nur dem äußerst möglichen Wortsinn des Gesetzeswortlauts nich widersprechen, ${ }^{22}$ ) wobei in unserem Fall diese Grenze nicht überschritten wurde. Ausgehend von dieser These muss notwendiger Weise zumindest auf Antrag eine Bescheidpflicht gem $\$ 410$ Abs 1 Z 7 ASVG bestehen, ${ }^{23}$ ) bei Säumnis bleibt die Möglichkeit der Säumnisklage. ${ }^{24}$ )

Man muss sich nun genauer fragen, was die Nachprüfung der Ermessensbindung bei den Pflichtaufgaben bedeutet: Wir haben nämlich im Bereich der

$\overline{\text { DRdA 2004/22 }}, 263($ Naderhirn $)=$ ZAS 2004/31, 183 (Haslinger $)=$ SZ 2003/14 $=$ SSV-NF $17 / 17$.

19) Herrschende Judikatur, vgl etwa mwN F. Bydlinski in Rummel, $\mathrm{ABGB}^{3} \$ 6 \mathrm{Rz} 21$ und Posch/Schwimann, $\mathrm{ABGB}^{3} \$ 6 \mathrm{Rz} 27$; vgl etwa auch Kramer, Juristische Methodenlehre $^{2}(2005) 89 \mathrm{ff}$.

$\left.{ }^{20}\right) \mathrm{AaO}$ 68; freilich ist die Gemeindeselbstverwaltung verfassungsrechtlich geregelt, während die gesetzliche SV einfachgesetzlich im Wege der Selbstverwaltung eingerichtet ist.

1) Kramer, Juristische Methodenlehre $123 \mathrm{ff}$

22) OGH 9. 11. 1993, 5 Ob 86/93 JBl 1994, 473 = EvBl 1994/92

$\left.{ }^{23}\right) \$ 410$ Abs 1 Z 7 ASVG.

$\left.{ }^{24}\right) \$ 67$ Abs 1 Z 2 ASGG.
Pflichtaufgaben aus Trägersicht wie bei den Pflichtleistungen eine Pflicht zur Leistung innerhalb der gesetzlichen Vorgaben. Der einzige Unterschied zu den Pflichtleistungen liegt darin, dass der Versicherte nach dem Willen der Gesetzesmaterialien keinen Rechtsanspruch auf die Leistung haben soll. Das in $\$ 154$ a ASVG postulierte pflichtgemäße Ermessen des Trägers wird durch den ebenfalls in dieser Bestimmung angeführten Leistungskatalog und den angeführten Zweck der Leistungen ${ }^{25}$ ) ganz entscheidend eingeengt. Verlangt nun aber eine verfassungskonforme Interpretation einen Instanzenzug und ein subjektives Recht auf Prüfung der Gesetzmäßigkeit der behördlichen Entscheidung, erweist sich die Konstruktion der Pflichtaufgabe irreführend: Wir haben - was den Prüfungsmaßstab betrifft - im Ergebnis die gleiche Rechtslage wie bei jenen Pflichtleistungen, wo in gleicher Weise ein bestehendes Ermessen der Behörde iSd Gesetzes auszuüben ist. Höchstens indirekt gesteht dies auch der OGH zu, wenn er bei der Ermessensprüfung verlangt, dass für Pflichtaufgaben zu prüfen ist, ob iSd $\$ 133$ Abs 2 ASVG die Behandlung ausreichend und zweckmäßig ist und das Maß des Notwendigen nicht überschritten hat. ${ }^{26}$ ) Freilich stellt sich die Frage, wo hier die Behörde überhaupt noch Ermessen hat, muss sie doch nach den Leitlinien des $\$ 133$ Abs 2 ASVG entscheiden, was eher in Richtung einer gebundenen Entscheidung sprechen könnte.

Dies führt aus Sicht des Versicherten zu einer weitgehenden Gleichbehandlung von Pflichtaufgaben und Pflichtleistungen. Die vom OGH eingeräumte Prüfungskompetenz geht über eine bloße Ermessensprüfung hinaus. Im Zuge der sukzessiven Kompetenz verliert nämlich der SV-Träger seine Entscheidungszuständigkeit und das Gericht entscheidet anstelle des Trägers neu. Eine Bindung des Gerichts an eine vorhergehende Ermessensübung der Behörde ist im Hinblick auf das Gewaltentrennungsprinzip ${ }^{27}$ ) ausgeschlossen. Daher erweist sich aus dem Blickwinkel des OGH die Pflichtaufgabe medizinische Rehabilitation in Wahrheit als Pflichtleistung mit Bescheidpflicht (auf Antrag).

\section{Geld- und Sachleistungen im Zusammenhang mit Rehabilitation}

\subsection{Teilversicherung in der gesetzlichen Unfallversicherung}

Das ASVG sieht eine eigene Teilversicherung in der UV in $\$ 8 \mathrm{Abs} 1 \mathrm{Z} 3$ lit c ASVG für bestimmte Fälle der medizinischen Rehabilitation vor. Das ASVG spricht insofern von einer Teilversicherung jener „Personen, die in einer Einrichtung untergebracht sind, die der medizinischen Rehabilitation oder Gesundheitsvorsorge dienen“. Nun wäre zwar eine Ambulanz eines KV-Trägers eine Einrichtung, die der medizinischen Rehabilitation dient. Der in $\$ 8$ ASVG verwendete Begriff der „Unterbringung" in einer solchen Anstalt schließt allerdings nach Ansicht der Höchstgerichte ein Element der Dauer ein, das im Fall kurzfris-

${ }^{25)}$ So verweist ja $\$ 154$ a Abs 1 ASVG auf $\$ 133$ Abs 2 ASVG.

${ }^{26}$ ) OGH 18.2.2003, 10 ObS 258/02t DRdA 2004/22, 263 (Naderhirn) $=$ ZAS 2004/31, 183 (Haslinger) $=$ SZ 2003/14 = SSV-NF 17/17.

${ }^{27)}$ Art $94 \mathrm{~B}-\mathrm{VG}$ 
tiger Aufenthalte nicht gegeben ist. ${ }^{28}$ ) Damit stehen nur stationäre Aufenthalte 列 der gesetzlichen UV. Wer diese Unterbringung finanziert, ist nach dem Wortlaut des $\$ 8$ Abs 1 Z 3 lit c ASVG irrelevant, sodass etwa auch der Aufenthalt eines Selbstzahlers diese Teilversicherung auslöst. Der Normzweck geht offenbar dahin, aufgrund der großen gesellschaftspolitischen Bedeutung der medizinischen Rehabilitation die Aufenthaltsrisiken stationärer Pflege sozial abzusichern.

\subsection{Geldleistungen während Maßnahmen}

\section{der medizinischen Rehabilitation?}

Inwieweit kann die Teilnahme an einer Maßnahme der medizinischen Rehabilitation Geldleistungen in der gesetzlichen SV auslösen. Einzugehen ist im Folgenden auf die Leistungen Pflegegeld, Krankengeld, Übergangsgeld, Zuschuss an den DG sowie die Pension wegen geminderter Arbeitsfähigkeit.

\subsubsection{Berücksichtigung bei der Pflegegeldbemessung}

Maßnahmen der medizinischen Rehabilitation können bei der Dauer der Zuerkennung von Pflegegeld eine Rolle spielen.

Es ist zulässig, dass das Pflegegeld befristet gewährt wird, wenn im Entscheidungszeitpunkt bereits "der Wegfall einer Voraussetzung für die Gewährun eines Pflegegeldes mit Sicherheit oder sehr hoher Wahrscheinlichkeit festgestellt werden kann" ( $\$ 9$ Abs 2 BPGG). Dieser Fall liegt zB vor, wenn nach einem Unfall eine erfolgreiche Rehabilitation zu erwarten ist. ${ }^{29}$ )

Erfolgt bei einer medizinischen Rehabilitation eine stationäre Aufnahme, so erhält der Pflegebedürftige dort umfassende Betreuungs- und Hilfsmaßnahmen ${ }^{30}$ ), weshalb der Anspruch auf Pflegegeld grundsätzlich „ab dem Tag, der auf die Aufnahme folgt" ruht ( $\$ 12$ Abs 1 Z 1 BPGG), sofern der stationäre Aufenthalt von der SV, dem Landesgesundheitsfonds oder bestimmten anderen öffentlichen Institution überwiegend finanziert wird. Dazu finden wird in $\$ 12$ Abs 3 BPGG wieder Gegenausnahmen. ${ }^{31}$

$\left.{ }^{28}\right)$ VwGH 24. 6. 1997, 95/08/0064 SVSlg 44.902; OGH 28. 4. 1998, 10 ObS 18/98i SSV-NF $12 / 57$.

Vgl Greifeneder/Liebhart, Pflegegeld ${ }^{2}$ (2008) Rz 216

$\left.{ }^{30}\right)$ Vgl Greifeneder/Liebhart, Pflegegeld ${ }^{2} \mathrm{Rz} 238$

1) Pflegegeld ist auf Antrag weiterhin zu leisten, wenn trotz des stationären Aufenthalts „pflegebedingte Aufwendungen nachgewiesen werden“. Das Pflegegeld gebührt in dem Umfang dieser Aufwendungen für höchstens drei Monate (ausnahmsweise länger, wenn dadurch für den Pflegegebedürftigen eine besondere Härte vermieden wird“ - soziä Här verhältnis des Pflegegeldbeziehers mit der Pflegeperson besteht, das dem ASVG unterliegt oder der Tatbestand gem $\$ 2$ Abs 1 Z 4 GSVG erfüllt wird, oder wenn sich die pflegebedingten Aufwendungen aus dem vertraglichen Betreuungsverhältnis des Pflegegeldbeziehers oder seines Angehörigen gem $\$ 1$ Abs 2 HBeG oder gem $\$ 159$ GewO 1994 ergeben. Eine Weiterleistung des Pflegegeldes findet auf Antrag in dem Umfang der Beitragshöhe für die Weitervericherung bzw Selbstversicherung der Pflegeperson statt $(\mathrm{vgl} \$ 12 \mathrm{Abs} 1$
Insb auch für ambulante Maßnahmen der medizinischen Rehabilitation stellt sich die Frage, ob diese bei der Bemessung des zeitlichen Pflegeaufwandes nach dem BPGG zu berücksichtigen sind. Nach der herrschenden Rechtspre chung liegt ein Pflegeaufwand nur vor, wenn die Maßnahme auch ein - ansonsten - nicht behinderter Mensch gewöhnlich selbst vornehmen kann. ${ }^{32}$ ) Nicht zu berücksichtigen sind nach der Judikatur auch Therapien, die der Erhaltung oder Verbesserung des Gesundheitszustands dienen und die etwa von Familienangehörigen nach erfolgter Einschulung durch Fachkräfte selbständig durchgeführ werden. ${ }^{33}$ ) Diese $\mathrm{mE}$ nicht ohne weiteres nachvollziehbare Judikatur ist in der Lehre auf Kritik gestoßen. ${ }^{34}$ )

\subsubsection{Krankengeld}

Nun ist zu prüfen, welche einkommensersetzenden Geldleistungen aus der gesetzlichen SV der Versicherte im Fall einer Sachleistung der medizinischen Rehabilitation beanspruchen kann. Solche Geldleistungen kommen in Betracht wenn durch die Rehabilitationsmaßnahme ein Einkommensausfall entsteh (etwa Wegfall von Arbeitslosengeld, Auslaufen des Anspruchs auf Entgeltfortzahlung gegenüber dem AG). Der Bezug einer Pension aus der gesetzlichen PV wird alleine durch die Teilnahme an einer Maßnahme der medizinischen Rehabilitation aus der gesetzlichen KV nicht berührt. Abweichendes gilt freilich, wenn die Rehabilitationsmaßnahme aus der gesetzlichen PV gewährt wird und die Pension noch nicht angefallen ist. ${ }^{35}$ )

Z 1 und Abs 3 Z 2 BPGG). Wird auch die Pflegeperson als Begleitperson stationär aufgenommen, weil ansonsten der stationäre Aufenthalt des Pflegebedürftigen nicht möglich wäre, so gebührt während deren stationären Aufenthalts auf Antrag das Pflegegeld ( $\$ 12$ Abs 3 Z 3 BPGG). Dies kann zB notwendig sein, wenn erforderliche Pflegepersonen in den Einrichtungen nicht vorhanden sind (insb bei Kuraufenthalten) (RV 1186 BlgNR 20 GP 14). Das Pflegegeld ist auf Antrag auch für jene stationär aufgenommene Pflegeperson $\mathrm{zu}$ leisten, die ein pflegebedürftiges Kind, einen pflegebedürftigen unmündigen Minderjährigen oder geistig Behinderten betreut und der stationäre Aufenthalt der Pflegeperson im Interesse des Pflegebedürftigen erforderlich ist (besser Aussichten auf Genesung, Gewährleistung intensiverer Betreuungsmaßnahmen - vgl RV 1186 BlgNR 20. GP 14).

${ }^{32}$ ) Greifeneder/Liebhart, Pflegegeld ${ }^{2} \mathrm{Rz} 14$; OGH 25. 7.2000, $10 \mathrm{ObS} 206 / 00 \mathrm{t}$ SSV NF 14/95; 10.2. 2004, 10 ObS 269/03m SSV-NF 18/6 = SVSlg 54.586; 23. 11. 2004, 10 ObS $142 / 04 \mathrm{~m}$ SSV-NF $18 / 79=$ SVSlg 54.588 .

${ }^{33}$ ) Siehe zB OGH 31. 8. 1999, 10 ObS 158/99d SSV-NF 13/76; 5.2.2008, 10 ObS 10/08f zuvo 2008/48, 73 = ZAS-Judikatur 2008/134. Nach Ansicht des OGH liegt keine Betreuung oder Hilfe iSd BPGG vor zB bei Bobath-Therapie (OGH 13. 12. 1996, 10 ObS 2393/93a SSV-NF 10/130 = SVSlg 44.160) oder Vojta (OGH 28. 1. 1997, 10 ObS 2460/96d SSV-NF 11/7 = SVSlg 44.166), dies soll zT auch für psychische Unterstützungsmaßnahmen zur Therapie gelten (zB OGH 5.9.2000, 10 ObS 216/00p ARD 5193/11/2001, 5. 2. 2008, 10 ObS 10/08f zuvo 2008/48, 73 = ZAS-Judikatur 2008/134; krit dazu Tomandh ZAS 1999, $13 \mathrm{ff}$; siehe auch Pfeffer, ZAS 1998/11, $120 \mathrm{f}$.

${ }^{34}$ ) Pfeffer, DRdA 1998, 120 f; Tomandl, Einige grundsätzliche Überlegungen zum Pflegegeldanspruch, ZAS 1999, 13 ff.

${ }^{35}$ ) Insofern geht der Anspruch auf Übergangsgeld einer noch nicht angefallenen Pension wegen geminderter Arbeitsfähigkeit vor (Details vgl \$ 307 ASVG). Ansonsten 
Ist der Versicherte weder erwerbstätig noch Bezieher einer einkommensersetzenden Leistung aus der gesetzlichen SV, löst alleine die Teilnahme an einer Maßnahme der medizinischen Rehabilitation aus der gesetzlichen KV keine einkommensersetzenden Geldleistungen aus. Eine solche Leistung würde ja auch kein ausfallendes Einkommen ersetzen.

Kommt es durch die Maßnahme der medizinischen Rehabilitation dagegen zu einem Einkommensausfall, stellt sich die Frage, ob und inwieweit eine Geldleistung zu gebühren hat. Fällt der Versicherte in den Kreis der Anspruchsberechtigten gem $\$ 138$ ASVG, dann kommt ein Anspruch auf Krankengeld in Betracht. Dieser Anspruch besteht unbestritten dann, wenn zugleich Arbeitsunfähigkeit wegen Krankheit besteht. Nun ist die Frage, was mit Versicherten ist, die zwar an sich arbeitsfähig wären, wo aber aufgrund einer Maßnahme der medizinischen Rehabilitation ein Arbeitsausfall eintritt. Gem $\$ 120$ Abs 1 Z 2 ASVG würde nämlich der Versicherungsfall erst mit der durch eine Krankheit herbeigeführten Arbeitsunfähigkeit eintreten, was gegen einen Anspruch auf Krankengeld spricht.

Die Praxis geht aber einen anderen Weg: Die Arbeitsunfähigkeit gem $\$ 120$ Abs 1 Z 2 ASVG wird parallel zur Regelung im EFZG verstanden und parallel zu Maßnahmen der medizinischen Rehabilitation soll auch ein Anspruch auf Krankengeld möglich sein. ${ }^{36}$ ) Fürs erste finden wir auch im Gesetz einen diesbezüglichen Hinweis, wenn in $\$ 306$ Abs 1 ASVG festgehalten wird, dass es Übergangsgeld für die Dauer der Gewährung von medizinischen Maßnahmen der Rehabilitation ab Beginn der 27. Woche nach dem letztmaligen Eintritt des Versicherungsfalls der Arbeitsunfähigkeit infolge Krankheit gibt. ${ }^{37}$ ) Offenbar geht diese Bestimmung davon aus, dass davor Krankengeld gebührt, denn Krankengeld gebührt gem $\$ 139$ Abs 1 ASVG für ein 26-wöchige Bezugszeit ab Beginn der Arbeitsunfähigkeit. Dies ist wohl auch der Grund, warum der OGH bei Aufenthalten in einem Kur- oder Erholungsheim eine der Krankenbehandlung vergleichbare Situation annimmt und Arbeitsunfähigkeit infolge Krankheit annimmt. ${ }^{38}$ ) Bei Aufenthalt in einem Kur- oder Erholungsheim nimmt der OGH das Vorliegen von Arbeitsunfähigkeit infolge Krankheit an, ohne dass es hiezu einer Krankschreibung durch den behandelnden Arzt bedarf. Insofern besteht
offenbar Gleichklang mit der arbeitsrechtlichen Beurteilung der Arbeitsunfähigkeit wegen Krankheit. ${ }^{39}$ ) In der nötigen Klarheit können wir diese Lösung aus dem ASVG nicht ableiten. Das tragende Begründungselement ist die beim Kran-

bleibt es beim Pensionsbezug: Die Pension soll den Lebensunterhalt in gleicher Weise garantieren, wie dies vor Einleitung der Rehabilitationsmaßnahme der Fall war und demgaran 15. 7. 2003, 10 ObS 189/03x infas 2004 S I = ARD 5510/13/2004).

${ }^{36}$ ) Vgl die in SozSi 1982, 328 abgedruckte Rechtsmeinung, die offenbar jene des HV ist und auf die sich auch der OGH bezieht (OGH 24. 8. 1993, 10 ObS 159/93 SSV-NF 7/76).

${ }^{37}$ ) Dann wird $\$ 143$ Abs 1 Z 4 ASVG interessant und der Anspruch auf Krankengeld ruht (zur Gänze).

${ }^{38}$ ) OGH 6. 12. 1994,10 ObS 281/94 SVSlg $42.364=$ SSV-NF $8 / 120$

${ }^{39}$ ) OGH 24. 8. 1993, 10 ObS 159/93 SSV-NF 7/76. kengeld bestehende Verzahnung bzw Parallelität mit den arbeitsrechtlichen Vorschriften zur Entgeltfortzahlung im Krankheitsfall.

Wird die Maßnahme der medizinischen Rehabilitation einem Arbeitslosengeld- oder Notstandshilfebezieher aus dem Bezug einer Leistung aus der Arbeitslosenversicherung heraus gewährt, gebührt diesem Krankengeld aufgrund seiner Pflichtversicherung in der KV als Leistungsbezieher nach dem AlVG. ${ }^{40}$ )

\subsection{3 Übergangsgeld}

Soweit Maßnahmen der medizinischen Rehabilitation als Leistung der PV gewährt werden, gebührt Übergangsgeld ( $\$ 306$ Abs 1 ASVG), ${ }^{41}$ ) welches zum Ruhen des Krankengeldanspruchs führt. ${ }^{42}$ ) Im Fall eines Anspruchs auf Übergangsgeld aus der PV gebührt dieses aber gem $\$ 306$ Abs 1 ASVG erst nach den ersten 26 Wochen (also nach der typischen Krankengeldbezugsphase). Auch in der UV besteht eine Subsidiarität des Übergangsgeldbezugs gegenüber anderen Leistungen, insb dem Anspruch auf Entgeltfortzahlung und jenem auf eine Pension wegen geminderter Arbeitsfähigkeit. ${ }^{43}$ )

\subsubsection{Zuschuss an den Dienstgeber gem § 53b ASVG?}

$\$ 53 \mathrm{~b}$ ASVG sieht - systematisch etwas versteckt im Beitragsrecht - eine Leistung der gesetzlichen $U^{44}$ ) vor in Form eines Zuschusses für DG hinsichtlich ihrer Aufwendungen an Entgeltfortzahlung bei Krankheit und Unfall. Nun stellt sich die Frage, ob im Fall einer Entgeltfortzahlung während einer Maßnahme der medizinischen Rehabilitation Anspruch auf diesen Zuschuss besteht. Weder im Gesetzestext noch in den Materialien findet man diesbezüglich einen Hinweis, auch die bisherige Judikatur ist auf diese Facette noch nicht eingegangen. In der Literatur wird freilich von Melzer-Azodanloo zu Recht darauf hingewiesen, dass ungeachtet der fehlenden Definition von „Krankheit“ und "Unfall“ in $\$ 53 \mathrm{~b}$ ASVG davon auszugehend ist, dass der enge systematische Konnex zu den arbeitsrechtlichen Entgeltfortzahlungsvorschriften es nahe legt, auf die dort verwendeten Definitionen zurück zu greifen. ${ }^{45}$ ) Soweit daher Entgeltfortzahlung gem $\$ 2$ EFZG bzw gem $\$ 8$ Abs 1 und 2 AngG gebührt, kommt auch ein $Z u$ schuss für den DG gem $\$ 53 \mathrm{~b}$ ASVG in Betracht.

$\left.{ }^{40}\right) \$ 41$ AlVG

${ }^{41}$ ) Übergangsgeld in der UV gibt es nur für die Dauer einer Ausbildung ( $\$ 198$ ASVG), wobei aber die einen Teil der Unfallheilbehandlung bildende medizinische Rehabilitation durchaus einen (subsidiären) Anspruch auf Familien- und Taggeld ( $\$ 195$ ASVG) auslösen kann. Allenfalls kann auch die freiwillige Leistung einer besonderen Unterstützung gem $\$ 196$ ASVG während der Unfallheilbehandlung gewährt werden.

$\left.{ }^{42}\right) \$ 143$ Abs 1 Z 4 ASVG

${ }^{43)} § 199$ Abs 3 ASVG.

$\left.{ }^{44}\right) \mathrm{Vgl}$ auch die Aufnahme in die Auflistung des $\$ 173 \mathrm{Z} 3$ ASVG.

${ }^{45}$ ) Zuschuss an Dienstgeber zur Entgeltfortzahlung bei Krankheit oder Unfall, ecolex 2006, 500 . 
3.2.5 Relevanz von Maßnahmen der medizinischen Rehabilitation für den Anspruch auf eine Pension wegen geminderter Arbeitsfähigkeit

Häufige Krankenstände und besondere Ruhepausen eines DN werden bei der Beurteilung der Berufsunfähigkeit bzw Invalidität in der PV mitberücksichtigt. Maßgeblich sind nicht die tatsächlichen, sondern die zu erwartenden Krankenstände. Ein Versicherter ist berufsunfähig, wenn damit zu rechnen ist, dass er wegen seines Gesundheitszustandes mindestens sieben Wochen pro Jahr im Krankenstand sein wird. ${ }^{46}$ ) Vorhersehbare Krankenstände im Ausmaß von $30 \mathrm{Ar}$ beitstagen schließen nicht vom allgemeinen Arbeitsmarkt aus. ${ }^{47}$ ) Es sind jedoch die Umstände des Einzelfalls zu berücksichtigen. Mehrmalige, nur kurz dauernde Krankenstände können vom DG organisatorisch leichter aufgefangen werden als lang dauernde zusammenhängende Krankenstände. ${ }^{48}$ ) Kann der DN seinen Arbeitsplatz nur aufgrund großen Entgegenkommens seitens des DG (hinsichtlich der zu erwartenden Krankenstände und zusätzlich nötigen Arbeitspausen) erhalten, schließt ihn dies vom allgemeinen Arbeitsmarkt aus. ${ }^{49}$ ) Vorhersehbare Maßnahmen der medizinischen Rehabilitation, insb stationäre Aufenthalte, werden bei dieser Beurteilung mit zu berücksichtigen und insofern krankheitsbedingten Absenzen gleichzuhalten sein. Bei bloß einmaligen Maßnahmen wird freilich eine solche Abwesenheit für die Zukunft gerade nicht anzunehmen sein und es werden diese daher aus diesem Grund bei der Prognose über noch zu erwartende Absenzen nicht einzurechnen sein.

\section{Arbeitsrechtliche Fragen}

\subsection{Entgeltfortzahlung durch den Arbeitgeber}

Liegt zugleich mit der Gewährung einer Maßnahme der medizinischen Rehabilitation auch Arbeitsunfähigkeit infolge Krankheit vor, hat der AN Anspruch auf Lohnfortzahlung gegenüber dem AG. Diese hat ihre Rechtsgrundiage für Angestellte in $\$ 8 \mathrm{AngG}_{\text {und }}{ }^{50}$ ) für Arbeiter im EFZG. Es gelangen somit die gesetzlichen Regeln zur Entgeltfortzahlung zur Anwendung, die im Allgemeinen hier nicht vertieft werden müssen.

Denkbar ist aber auch, dass eine stationäre oder ambulante Maßnahme de medizinischen Rehabilitation erforderlich wird und aufgrund dessen der AN seiner Arbeit nicht nachkommen kann, obwohl er an sich arbeitsfähig wäre. Dieser besondere Fall ist hinsichtlich der stationären Aufenthalte ausdrücklich gesetzlich geregelt, allerdings kurioserweise nur im EFZG für die Arbeiter.

Klar ist damit die Rechtslage bei den Arbeitern geregelt: Anders als das AngG stellt $\$ 2$ Abs 2 EFZG klar, dass medizinisch indizierte Aufenthalte in Kur-

46) ZB OGH 14. 1. 1991, 10 ObS 361/91 SSV-NF 6/3 = SVSlg 38.327 und 16. 6. 1992 10 ObS $119 / 92$ SSV-NF $6 / 70=$ SVSlg 38.330

${ }^{47}$ ) OGH 12.9. 1989, 10 ObS 292/89 SSV-NF 3/107.

${ }^{48}$ ) OGH 12.9. 1989, 10 ObS 292/89 SSV-NF 3/107.

$\left.{ }^{49}\right) \mathrm{HA}$, vgl etwa Resch, Sozialrecht ${ }^{4}$ (2008) 120.

${ }^{50}$ ) Allerdings unverständlicher Weise inhaltlich abweichend. oder Erholungsheimen, die der Erhaltung, Besserung oder Wiederherstellung der Arbeitsfähigkeit dienen, einer Krankheit gleichzuhalten sind. Erforderlich ist dass die Aufenthalte durch einen SV-Träger bewilligt oder angeordnet werden wobei Zuzahlungen des Versicherten unschädlich sind. Nach $\$ 2$ Abs 7 EFZG muss der Zuschuss des Trägers aber eine bestimmte Mindesthöhe ${ }^{51}$ ) erreichen..$^{52}$ Der Gesetzgeber hat damit einen Grundsatz, der von der Judikatur zu $\$ 8$ AngG entwickelt worden ist, in das EFZG ausdrücklich aufgenommen..$^{53}$ ) Damit gelten auch Zeiten einer Arbeitsverhinderung, die nicht durch eine akute Krankheit, sondern durch bestimmte Maßnahmen der Gesundheitsvorsorge oder Rehabilitation bedingt sind, als Arbeitsverhinderung durch Krankheit, wobei die Dienstverhinderungszeiten auf das Höchstausmaß des Entgeltfortzahlungsanspruch anzurechnen sind. ${ }^{54}$ ) Erfolgt allerdings auf Anordnung des SV-Trägers kein Aufenthalt in einem Kur- oder Erholungsheim, sondern etwa die schlichte Unterbringung in einem privaten Quartier auf dem Land zur Erholung ${ }^{55}$ ) besteh nach dem Wortlaut des $\$ 2$ Abs 2 EFZG kein Anspruch auf Entgeltfortzahlung. Bei Schaffung des EFZG dürfte der Gesetzgeber freilich nicht das mit der 50 . ASVG-Novelle geschaffene Spektrum möglicher Maßnahmen der medizinischen Rehabilitation vorausgeahnt haben: Dies spricht dafür, dass man insofern von einer nachträglichen Lücke im $\$ 2$ Abs 2 EFZG ausgeht, die mit einer Analogie zu $\$ 2$ Abs 7 EFZG zu schließen wäre.

Das Fehlen einer ausdrücklichen Regelung für die Angestellten im AngG veranlasst die Lehre zu einer Gleichstellung mit $\$ 2$ Abs 2 EFZG. ${ }^{56}$ ) Methodologisch richtig folgert Drs eine Analogie zu dieser Bestimmung. ${ }^{57}$ ) Schon vor Schaffung des EFZG wurde der Gleichklang von der Judikatur ausgesprochen..$^{58}$ )

Soweit weder Arbeitsunfähigkeit wegen Krankheit noch eine Unterbringung in einer der in $\$ 2$ Abs 2 EFZG genannten Einrichtungen vorliegt, greifen die gesetzlichen Vorschriften zur Lohnfortzahlung wegen Krankheit nicht. Bei kurzen Verhinderungen mag ein sonstiger wichtiger die Person des AN betreffender Grund vorliegen, der eine Verhinderung an der Leistung der Dienste wäh-

$\left.{ }^{51}\right)$ Es genügt, wenn ein Kostenzuschuss mindestens in der halben Höhe der gemäß $\$ 45$ Abs 1 ASVG geltenden Höchstbeitragsgrundlage für jeden Tag des Aufenthaltes gewährt wird (also im Jahr 2009 ein Betrag von $€$ 67,-- täglich).

52) Drs in ZellKomm \$2 EFZG Rz 22

53) Cerny/Kallab, EFZG 80 ( $\$ 2$ Anm 15) unter Hinweis auf die Materialien (1105 BlgNR 13. GP 13).

${ }^{54}$ ) Cerny/Kallab, aaO.

${ }^{55}$ ) Etwa in Form eines Genesungsaufenthalts gem $\$ 6$ RRK 2005, eines Erholungsaufenthalts für Mütter mit Kleinkindern gem $\$ 7$ RRK 2005, Erholungsaufenthalte (\$\$ 8 f RRK 2005) oder Landaufenthalte (\$10 RRK 2005).

$\left.{ }^{56}\right) \mathrm{MwN}$ etwa Holzer in Marhold/Burgstaller/Preyer (Hrsg), AngG-Kommentar (Loseblattausgabe Stand 2008) \$8 Rz 14; Drs in ZellKomm \$8 AngG Rz 19; Melzer Azodanloo in Löschnigg (Hrsg) AngG I ${ }^{8}(2007) \$ 8 \mathrm{Rz} 52$

$\left.{ }^{57}\right)$ Drs in Zell Komm $\$ 8$ AngG Rz 19

${ }^{58}$ ) OGH 20. 9. 1962, 4 Ob 97/62 Arb 7652 = JBl 1963, 164 (zust F. Bydlinski) (ärztlich angeordnete Kurbehandlung in einem auswärtigen Badeort gegen chronisch entzündliche Veränderungen der Schleimhaut, des Rachens und des Kehlkopfes); 24. 8. 1993, 10 ObS 159/93 SSV-NF 7/76 = DRdA 1994, $172=$ infas 1994 S 29. 
rend einer verhältnismäßig kurzen Zeit rechtfertigt ( $\$ 8$ Abs 3 AngG bzw $\$ 1154 \mathrm{~b}$ ABGB). So werden ambulante Maßnahmen der medizinischen Rehabilitation als solche sonstigen Dienstverhinderungsgründe aus wichtigem Grund zu qualifizieren sein, wenn nicht überhaupt dem Versicherten zugemutet wird, die Maßnahme in der Freizeit durchzuführen. ${ }^{59}$ ) Im Übrigen bleibt es beim Grundsatz, das mangels Arbeitsleistung des AN auch die Entgeltpflicht des AG entfällt. Allenfalls können Geldleistungen aus der gesetzlichen SV greifen.

\subsection{Schicksal des aufrechten Arbeitsvertrags bei Auslaufen der Entgeltfortzahlung}

Sind die gesetzlichen Fristen zur Lohnfortzahlung im Krankheitsfall ausgelaufen, haben wir ein aufrechtes Arbeitsverhältnis, bei dem ähnlich einer Karenzierung die wechselseitigen Pflichten Arbeitsleistung und Entgeltzahlung aufgehoben sind. Wenn nun die Entgeltfortzahlung aufgehoben ist, wäre der AG grundsätzlich einmal durch die Absenz des AN wirtschaftlich nicht belastet (wenn man vom Erfordernis der Bestellung einer Ersatzarbeitskraft absieht).

Dem ist freilich bei genauerer Betrachtung nicht so:

Für alle AN entsteht mit jedem Arbeitsjahr ein neuer Anspruch auf bezahlten Erholungsurlaub ( $\$ 2$ Abs 2 S 2 UrlG) sowie für die Dauer des Krankengeldbezugs auch eine Beitragspflicht des AG zur „Abfertigung neu“ ( $\$ 7$ Abs 3 BSMVG)

Auch die Entgeltfortzahlung bei Krankheit ist bei den Arbeitern insofern nicht gänzlich aufgehoben, als bei den Arbeitern mit dem neuen Arbeitsjahr ein nommt zu einem Hineingleiten in die neue Entgeltfortzahlungsperiode, die neuerlich zur Gänze in Anspruch genommen werden kann..$^{60}$ ) Dies sollte nach einer Entscheidung sogar dann gelten, wenn das Arbeitsverhältnis zu Beginn des neuen Arbeitsjahres bereits rechtmäBig beendet ist, aber nach dem EFZG noch Entgeltfortzahlung gebührt und diese Entgeltfortzahlung in das neue Arbeitsjahr hineinreicht. ${ }^{61}$ ) Auch in diesem Fall soll Anspruch auf den neuen Entgeltfortzahlungsanspruch für die Dauer der Krankheit bestehen. ${ }^{62}$ ) In der Lehre wurde daraus geschlossen, dass dies selbst für Folgejahre ad infinitum gelten soll, solange nur der Krankenstand andauert, mag auch der Arbeitsvertrag längst rechtmäßig beendet worden sein: Festzuhalten ist freilich, dass der OGH diesen Fall nicht zu entscheiden hatte und daher auch

${ }^{59}$ ) Drs, Sonstige Dienstverhinderungsgründe, in Resch (Hrsg), Fragen der Lohnfortzahlungspflicht des Arbeitsgebers (2004) $51 \mathrm{ff}$.

${ }^{60}$ ) Zur bisherigen Rechtsprechung vgl etwa Löschnigg, Arbeitsrechtsänderungsgesetz (ARÄG) 2000 - Dienstverhinderungen/Krankenstand und Freizeit während der Kündi(ARAG) (15, Reissner, Arbeitsvertragsbeendigung und Krankenstand, in Resch (Hrsg), Arbeitsvertragsbeendigung und Krankenstand (2007) $50 \mathrm{f}$; aus der aktuellen Judikatur etwa OGH OGH 17. 4. 1997, 8 ObA 163/98y ZAS 1999/18, 167 (Pernkopf) $=$ infas 1999 A 74 .

$\left.{ }^{61}\right)$ Dies greift gem $\$ 5$ EFZG bei Kündigung durch den AG, Entlassung ohne wichtigem Grund und Austritt ohne wichtigem Grund.

${ }^{62}$ ) OGH 7. 6. 2006, 9 ObA 115/05k DRdA 2007/24, 236 (Kallab). nicht diese $\mathrm{mE}$ frgw These einer Entgeltfortzahlung ad infinitum für chronisch Kranke trotz rechtmäßig beendetem Arbeitsvertrag vertreten hat. ${ }^{63}$ )

Erst jüngst hat der OGH dagegen seine bisherige Rechtsprechung verworfen, was die Entgeltfortzahlung für den Fall einer durchgehenden Dienstverhinderung wegen Arbeitsunfall oder Berufskrankheit bei Arbeitern betrifft: Neu ist eine Entscheidung zur Entgeltfortzahlung an Arbeiter bei Arbeitsunfall und Berufskrankheit nach $\$ 2$ Abs 5 EFZG: Es bleibt bei der acht- bzw zehnwöchigen Entgeltfortzahlung nach Arbeitsunfall bzw Berufskrankheit, selbst wenn die Dienstverhinderung in das neue Arbeitsjahr hineinreicht. Für einen neuerlichen Entgeltfortzahlungsanspruch wegen Arbeitsunfall bzw Berufskrankheit im neuen Arbeitsjahr ist bei wiederholter Arbeitsverhinderung erforderlich, dass der AN seine Arbeit zwischenzeitig wieder aufgenommen hat. ${ }^{64}$ ) Der wegen eines Arbeitsunfalls oder einer Berufskrankheit permanent kranke AN belastet den AG nach dieser Judikaturwende daher nicht mit zusätzlichen Entgeltfortzahlungskosten.

Denkbar wäre nun, dass der AG den Gesundheitszustand des AN und seine Dienstverhinderungen durch medizinische Rehabilitationsmaßnahmen und Krankenstand zum Anlass nimmt, das Arbeitsverhältnis zu beenden.

\subsection{Bestandsschutz}

4.3.1 Behinderung iSd $\$ 3$ BEinstG

Thema ist in diesem Zusammenhang, inwieweit sich der Rehabilitand auf den Diskriminierungsschutz für Behinderte nach den $\$ \$ 7 \mathrm{a}$ ff BEinstG stützen kann. Der dort geregelte Diskriminierungsschutz ist vom Aufbau dem GlBG vergleichbar und geht auf Art 13 EG und vor allem auf die Vorgaben der RL 2000/78/EG zurück und bringt ua einen Anfechtungstatbestand für den Fall einer diskriminierenden Kündigung bzw Entlassung wegen einer Behinderung. ${ }^{65}$ )

Freilich befindet sich der Teilnehmer an einer Maßnahme der medizinischen Rehabilitation gerade in einem Prozess der gesundheitlichen Besserung. Somit ist der maßgebliche Begriff der Behinderung näher zu untersuchen: $\$ 3$ $B E i n s t G$ definiert diese als „die Auswirkung einer nicht nur vorübergehenden körperlichen, geistigen oder psychischen Funktionsbeeinträchtigung oder Beeinträchtigung der Sinnesfunktionen, die geeignet ist, die Teilhabe am Arbeitsleben $\mathrm{zu}$ erschweren. Als nicht nur vorübergehend gilt ein Zeitraum von mehr als voraussichtlich sechs Monaten. ${ }^{\text {"66) }}$

${ }^{63}$ ) IdS aber Kallab, DRdA 2007, 238 und Reissner, aaO 51 (der aber meint, man müssen irgendwann sagen, dass der Zusammenhang mit dem seinerzeitigen Arbeitsverhältni nicht mehr gegeben ist bzw die Funktion von $\$ 8$ AngG bzw $\$ 5$ EFZG erledigt ist).

$\left.{ }^{64}\right)$ OGH 14. 10.2008, 8 ObA 44/08s unter Abkehr von seiner bisherigen Rechtsprechung OGH 9 ObA $13 / 06 \mathrm{~m}$.

$\left.{ }^{65}\right) \$ 7 \mathrm{f} \mathrm{BEinstG}$, der auch für Auflösungen in der Probezeit gilt und eine Sonderregelung für ein befristetes Arbeitsverhältnis vorsieht, sofern dieses auf die Umwandlung in ein unbefristetes Arbeitsverhältnis "angelegt ist“

${ }^{66}$ ) Zur Auslegung von $\$ 3$ BEinstG vgl (jeweils vor Kundmachung des EuGH Urteils in der Rs Navas) Brodil in Rebhahn (Hrsg), GlBG Gleichbehandlungsgesetz (2005) $684 \mathrm{ff}$ sowie K. Mayr in ZellKomm \$ 3 BEinstG Rz $1 \mathrm{ff}$ 
In diesem Zusammenhang finden wir eine Entscheidung des EuGH. ${ }^{67}$ ) Dieser hatte eine am 28.5. 2004 ausgesprochene krankheitsbedingte Kündigung eines bereits seit 14. 10. 2003 krankgeschriebenen AN vor dem Hintergrund der RL 2000/78 zu beurteilen. Die Vorlagefrage ging dahin, ob eine ausschließ lich wegen einer Krankheit ausgesprochene Kündigung unter die RL 2000/78/ EG fällt. Der EuGH hält fest, dass der Begriff der Behinderung iSd Art 1 der RL 2000/78/EG nicht nach nationalem Recht, sondern europaeinheitlich zu verstehen ist. $\left.{ }^{68}\right)$ Ziel der RL ist die Bekämpfung von Diskriminierung in Beschäftigung und Beruf, ua aufgrund der Behinderung. Behinderung ist so zu verstehen, das damit Einschränkungen erfasst sind, die insb auf physische, geistige oder psychische Beeinträchtigungen zurückzuführen sind und die ein Hindernis für die Teilhabe des Betreffenden am Berufsleben bildet. ${ }^{69}$ ) Damit wählt die RL bewuss ein Wort, das sich von dem der "Krankheit" unterscheidet und daher lassen sich die beiden Begriffe nach Ansicht des EuGH „nicht schlicht und einfach einander

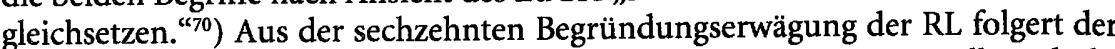
EuGH, dass der europäische Gesetzgeber bei der Behinderung an Fälle gedach hat, wo die Teilhabe am Berufsleben über einen langen Zeitraum eingeschränkt ist. Es muss daher bei der Einschränkung wahrscheinlich sein, dass sie von langer Dauer ist. Bezogen auf den Fall des EuGH hat er eine mehr als sechs Monate dauernde Krankheit noch nicht als ausreichend lang qualifiziert und fasst damit das zeitliche Element strenger wie die Sechsmonatsgrenze des die RL umsetzenden österreichischen BEinst $\left.\mathrm{G}^{71}\right)^{72}$ )

Der EuGH stellt fest, dass der EGV keine Bestimmung enthält, die die Diskriminierung wegen einer Krankheit als solcher verbietet. ${ }^{73}$ ) Die in der RL aufgezählten Diskriminierungsverbote hält er für abschließend und spricht sich gegen eine darüber hinaus gehende Interpretation (welche Krankheit mit einschließt) aus. ${ }^{74}$ ) Die Erklärung für diese restriktive Interpretation finden wir in den Schlussanträgen: ${ }^{75}$ ) Eine weite Auslegung des Art 13 EG und der RL „hätte zur Folge, dass sozusagen ein archimedischer Punkt geschaffen würde, von dem aus das in Art 13 EG umschriebene Diskriminierungsverbot gehandhabt werden könnte, um ohne Einschaltung des Vertragsgebers oder des Gemeinschaftsgesetzebers die Abwägungen zu korrigieren, die die Mitgliedstaaten" innerhalb ihrer noch verbleibenden Kompetenzen vorgenommen haben. ${ }^{76}$ )

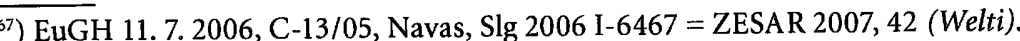

$\left.{ }^{68}\right) \mathrm{Rz} 39$ ff des Urteils.

${ }^{69)} \mathrm{Rz} 43$ des Urteils.

70) Rz 44 des Urteils.

$\left.{ }^{71}\right)$ Runggaldier, EuGH präzisiert den Diskriminierungstatbestand „Behinderung“ gem Art 13 EGV und RL 2007/78/EG, RdW 2006/546, 579.

${ }^{72}$ ) Eine solche nationale Regelung, die strenger zugunsten des Behinderten wirkt, ist gem Art 8 RL 2000/78/EG zulässig

$\left.{ }^{73}\right) \mathrm{Rz} 54$ des Urteils.

${ }^{74)} \mathrm{Rz} 56$ des Urteils.

${ }^{75}$ ) Schlussanträge des Generalanwalts Geelhoed vom 16. 3. 2006 (Slg 2006 I 6467).

76) Rz 54 der Schlussanträge.
In der Literatur wurde an der EuGH-Entscheidung zu Recht bemängelt, ${ }^{77}$ ) dass es der EuGH verabsäumt hat, den Zusammenhang zwischen Krankheit und Behinderung aufzuarbeiten, obwohl er auf die gesundheitswissenschaftlichen Erkenntnisse hingewiesen hat, wie sie der International Classification of Functioning, Disability and Health (ICF) der WHO zugrundeliegen. ${ }^{78}$ ) Dem Sachverhalt fehlten Angaben zu Art, Verlauf, Prognose und Auswirkungen der Gesundheitsstörung und zum Verhalten des AG. ${ }^{79}$ ) Zu Recht wird in der Kritik klargestellt, dass Krankheit und Behinderung nicht zwei scharf voneinander abzugrenzende Zustände und Tatbestände sind, sondern unterschiedliche Betrachtungsweisen von Gesundheitsproblemen darstellen. Welti stellt die naheliegende Frage, ob nicht jede Kündigung wegen Arbeitsunfähigkeit eine verbotene Benachteiligung darstellen müsste. Er rückt diese These aber sogleich zurecht: Auch die RL 2000/78/EG gebietet nicht, ein dauerhaft gestörtes Synallagma fortzusetzen. Sie verpflichtet aber die AG zu zumutbaren Anstrengungen, die Voraussetzungen des Arbeitsverhältnisses durch behindertengerechte Arbeitsplatzgestaltung herzustellen. ${ }^{80}$ )

Soweit die Maßnahme der medizinischen Rehabilitation dem Bereich der Krankheit zuzurechnen ist, kommt folglich dafür der Tatbestand der Behinderung und der damit verbundene Diskriminierungsschutz nach dem BEinstG grundsätzlich nicht zum Tragen. Es gibt freilich einen überschneidenden Bereich, so dass etwa chronische Krankheiten den nötigen Dauercharakter für eine Behinderung aufweisen können. Dann kann aufgrund von Charakter und Verlauf des Leidens zugleich auch eine Behinderung iSd RL 2000/78/EG und iSd $\$ 3$ BEinstG vorliegen, mit der Besonderheit, dass im österreichischen Recht gem $\$ 3$ BEinstG bereits sechs Monate Beeinträchtigung als Richtwert genügen.

\subsubsection{Gerechtfertigte oder unberechtigte Entlassung?}

Der Antritt einer Kur berechtigt den AG nicht zur Entlassung, insb ist der AN nicht verpflichtet, den Antrittstag einer vom SV-Träger verordneten Kur mit dem AG zu vereinbaren: ${ }^{81}$ ) Die Arbeitsverhinderung ist jedenfalls gerechtfertigt und es besteht ein Anspruch auf Entgeltfortzahlung.

Im medial Aufsehen erregenden Fall Alexander Sperr erachtete der OGH die Entlassung eines Berufsfussballspielers wegen eines Trainingsunfalls als berechtigt, weil aufgrund der erlittenen dauernden Lähmung dauernde Dienst- bzw Arbeitsunfähigkeit iSd $\$ 82$ lit b GewO 1959 bzw bei Annahme eines Angestellten iSd $\$ 27 \mathrm{Z} 2$ AngG vorgelegen ist. ${ }^{82}$ ) Dies bedeutet, dass unabhängig vom Grund der Dienstunfähigkeit, also etwa auch wegen einer Behinderung, in bestimm-

${ }^{77)}$ Welti, ZESAR 2007, 47

$\left.{ }^{78}\right) \mathrm{Rz} 22$ f des Urteils.

$\left.{ }^{79}\right)$ Welti, ZESAR 2007,47

${ }^{80}$ Welti, ZESAR 2007, $47 \mathrm{f}$

$\left.{ }^{81}\right)$ OGH 13. 9. 1995, 9 ObA 88/95 RdW 1996, $333=$ infas 1996 A $42=$ Arb 11.444

${ }^{82}$ ) OGH 13. 10. 1993, 9 ObA 186/93 ecolex 1994, $113=$ RdW 1994, $186=$ wbl 1984 $128=$ DRdA 1994/25, 320 (M. Binder) $=$ infas 1994 A $70=$ ZASB 1994, 6. 
ten Fällen eine berechtigte Entlassung ausgesprochen werden darf. ${ }^{83}$ ) Dauernde Dienst- bzw Arbeitsunfähigkeit berechtigt nach der Judikatur auch dann zur Entlassung, wenn diese durch Krankheit oder Unglücksfall hervorgerufen wurde, weil die Ursache für die Dienstunfähigkeit generell ohne Bedeutung sein soll: ${ }^{84}$ Das schafft einen gewissen Gegensatz zu $\$ 27$ Z 5 AngG, wonach Krankheiten oder Unglücksfälle keine Entlassung wegen der damit verbundenen Abwesenheit durch erhebliche Zeit rechtfertigen können. Diese Einschränkung soll insb auch für Kur- und Erholungsaufenthalte, Aufenthalte in Heil- und Pflegeanstalten usw gelten, soweit diese aus Gründen der Erhaltung, Besserung oder Widerherstellung der Arbeitsfähigkeit notwendig sind ${ }^{85}$ ) Alleine die Abwesenheit zur medizinischen Rehabilitation rechtfertigt daher keinesfalls die Entlassung wegen dauernder Dienstunfähigkeit. Das veranlasst etwa Friedrich zur These, das Krankheit und Unglücksfälle nur dann zur Entlassung wegen Dienstunfähigkeit berechtigen, wenn der Zustand des Angestellten die Erbringung weiterer vertragsgemäßer Dienstleistungen vollkommen ausschließt oder nicht vorhersehbar ist, ob der Angestellte überhaupt jemals wieder dienstfähig wird. ${ }^{86}$ )

\subsubsection{Kündigungs- und Entlassungsschutz}

Keine Besonderheiten gelten im Zusammenhang mit medizinischen Rehabilitationsmaßnahmen hinsichtlich Kündigungs- und Entlassungsschutz. Abgesehen vom allenfalls relevanten Kündigungsschutz für besonders geschützte Behinderte gem $\$ 8$ BEinstG, bleibt es bei den allgemeinen Vorschriften. ${ }^{87}$ ) Allenfalls kann auch die krankheits- oder gebrechensbedingte Minderleistung als in der Person begründete Rechtfertigung für eine Kündigung angesehen werden, wenn diese wegen Sozialwidrigkeit angefochten wird. ${ }^{88}$ )

\section{Zusammenfassung}

5.1 Ist eine Maßnahme von ihrer Art und ihrem Zweck sowohl als Krankenbehandlung iSd $\$ \$ 133 \mathrm{ff}$ ASVG mit Pflichtleistungsanspruch zu qualifizieren, als auch als Maßnahme der medizinischen Rehabilitation iSd $\$ 154$ a ASVG, muss - da mit der 50. ASVG-Novelle keine Einschränkung des Leistungsanspruchs intendiert war - in diesem überlappenden Bereich eine Pflichtleistung vorliegen. (2.1)

5.2 Die vom OGH vertretene Rechtskontrolle der Entscheidung des Trägers über die Pflichtaufgabe medizinische Rehabilitation bedeutet eine verfas-

${ }^{83)}$ Insofern ist der Judikatur mE zuzustimmen, ebenso mwN Gerharth, ASoK 2008, 28.

${ }^{84)}$ MwN Friedrich in Marhold/Burgstaller/Preyer, AngG-Kommentar (8. Lfg 2005) $\$ 27 \mathrm{Rz} 279$.

85) Pfeil in ZellKomm $\$ 27$ AngG Rz 151

${ }^{80}$ ) In Marhold/Burgstaller/Preyer, AngG $\$ 27 \mathrm{Rz} 281$

$\left.{ }^{87}\right)$ MwN zuletzt etwa Gerhartl, ASoK 2008, $25 \mathrm{ff}$.

${ }^{88}$ ) MwN etwa Wolligger in ZellKomm $\$ 105$ ArbVG Rz 196 ff; zur Auflösung des Arbeitsvertrags bei Krankheit vgl auch die ausführliche Untersuchung von Reissner, Arbeitsvertragsbeendigung und Krankenstand, in Resch (Hrsg), Arbeitsvertragsbeendigung und Krankenstand (2007) $43 \mathrm{ff}$ sungskonforme Interpretation des $\$ 154 a$ ASVG. Weil im Rahmen der sukzessiven Kompetenz das Arbeits- und Sozialgericht jedenfalls neu entscheiden muss, entscheidet das Gericht ohne Bindung an die behördliche Vorentscheidung neu. (2.2)

5.3 Eine Teilversicherung in der UV besteht für die Dauer einer stationären Behandlung zur medizinischen Rehabilitation, die unabhängig davon greift, wer die Maßnahme finanziert. (3.1)

5.4 Der Anspruch auf Pflegegeld ruht idR bei stationärer Behandlung. Speziell für ambulante Behandlungen ist die Judikatur des OGH zu beachten, wonach ein Pflegeaufwand nur dann vorliegt, wenn die Maßnahme auch ein - ansonsten - nicht behinderter Mensch gewöhnlich selbst vornehmen kann. Nicht zu berücksichtigen sind nach Ansicht des OGH Therapien, die der Erhaltung oder Verbesserung des Gesundheitszustands dienen und die etwa von Familienangehörigen nach erfolgter Einschulung durch Fachkräfte selbständig durchgeführt werden. (3.2.1)

5.5 Aus der Parallelität zum Entgeltfortzahlungsrecht im Arbeitsrecht wird gefolgert, dass auch stationäre medizinische Rehabilitationsmaßnahmen in der KV selbst dann den Versicherungsfall der Arbeitsunfähigkeit wegen Krankheit (und damit den Krankengeldanspruch) auslösen, wenn der Versicherte an sich arbeitsfähig ist. (3.2.2)

5.6 Allenfalls kann bei Leistungen der medizinischen Rehabilitation aus der PV und UV Anspruch auf Ubergangsgeld bestehen (3.2.3). Gem $\$ 53 \mathrm{~b}$ ASVG könnte der DG einen Zuschuss gem §53b ASVG beanspruchen (3.2.4). Wohl nur ausnahmsweise können Zeiten der Arbeitsunfähigkeit wegen Maßnahmen der medizinischen Rehabilitation auch bei der Frage zu berücksichtigen sein, ob Anspruch auf eine Pension wegen geminderter Arbeitsfähigkeit besteht (3.2.5)

5.7 Selbst wenn im Rechtssinn keine Arbeitsunfähigkeit besteht, besteht gegenüber dem AG Anspruch auf Entgeltfortzahlung bei stationären medizinischen Rehabilitationsmaßnahmen, sofern diese in einem Kur-oder Erholungsheim iSd $\$ 2$ Abs 2 EFZG erfolgen. Dies gilt analog auch für Angestellte. Liegt weder Arbeitsunfähigkeit wegen Krankheit noch eine Unterbringung in einer der in $\$ 2$ Abs 2 EFZG genannten Einrichtungen vor, gebührt keine Lohnfortzahlung wegen Krankheit. Bei kurzen Verhinderungen mag ein sonstiger wichtiger die Person des AN betreffender Grund vorliegen, der eine bezahlte Dienstverhinderung während einer verhältnismäßig kurzen Zeit rechtfertigt ( $\$ 8$ AngG bzw $\$ 1154$ b ABGB). (4.1)

5.8 Sind die gesetzlichen Fristen zur Lohnfortzahlung im Krankheitsfall ausgelaufen, haben wir ein aufrechtes Arbeitsverhältnis, bei dem ähnlich einer Karenzierung die wechselseitigen Pflichten Arbeitsleistung und Entgeltzahlung aufgehoben sind. Dabei gelten aber Ausnahmen: Mit Erreichen eines neuen Arbeitsjahres entsteht bei Arbeitern ein neuerlicher Entgeltfortzahlungsanspruch, was aber nicht für die gesonderte Entgeltfortzahlungfrist der Arbeiter bei Arbeitsunfall oder Berufskrankheit gilt. Weiters entsteht mit dem neuen Urlaubsjahr ein neuer Anspruch auf bezahlten Erholungsurlaub ( $\$ 2 \mathrm{Abs} 2 \mathrm{UrlG}$ ) und für die Dauer des Krankengeldbezugs greift bei aufrechtem Arbeitsvertrag auch eine Beitragspflicht des AG zur „Abfertigung neu“ ( $\$ 7$ Abs 3 BSMVG). (4.2) 
5.9 Der Diskriminierungsschutz bei Behinderung nach dem BEinstG erfasst nach der Judikatur des EuGH nicht den Fall der Krankheit, wobei aber in Bezug auf Maßnahmen der medizinischen Rehabilitation Überschneidungen denkbar sind (4.3.1). Nur ausnahmsweise wird aufgrund der gesundheitlichen Beeinträchtigung ein Entlassungsgrund vorliegen (4.3.2). Im Übrigen gelten für eine Beendigung des Arbeitsvertrags während einer Maßnahme der medizinischen Rehabilitation die allgemeinen Vorschriften des Beendigungsrechts (4.3.3).
Hans Peter Spiegl

\section{Ausgewählte leistungsrechtliche Probleme im Zusammenhang mit Rehabilitation - ausgewählte leistungsrechtliche Probleme in Deutschland}

\section{Einleitung}

In meinem Vortrag über die leistungsrechtlichen Probleme der medizinischen Rehabilitation in Deutschland möchte ich mein Augenmerk gezielt auf eine Reihe von Fragen richten, die in den letzten Jahren in der Rechtsprechung und in der Folge in der Literatur eine besondere Rolle gespielt haben. Die dabei erkennbaren Problemstellungen lassen sich dabei weitgehend zwei Gesichtspunkten zuordnen, die sich gleichsam wie ein roter Faden durch den Vortrag ziehen, nämlich:

- Das Verhältnis des 9. Buches des SGB - Rehabilitation und Teilhabe behinderter Menschen (SGB IX) - zu den einzelnen Leistungsgesetzen und

- wie bewähren sich die neu im SGB IX enthaltenen Instrumente? Stichworte hierzu sind etwa „persönliches Budget“ bzw „Wahl- und Wunschrecht des Versicherten".

\section{Anspruch auf Rehabilitation}

Das SGB IX fasst die für die Rehabilitation und Teilhabe behinderter Menschen geltenden Rechtsvorschriften bereichsübergreifend und einheitlich zusammen. Der Gesetzgeber des SGB IX hat dabei zunächst an dem im Laufe der Jahrzehnte gewachsenen stark gegliederten System der Rehabilitation festgehalten mit der Folge, dass sich Voraussetzungen, Art und Umfang der Leistungen zur Rehabilitation nach den Rechtsvorschriften des im Rahmen des gegliederten Systems zuständigen Leistungsträgers richten, andererseits wurde ein fachübergreifender einheitlicher Rechtsrahmen geschaffen.

Wesentlich für die Bestimmung der Abgrenzung des SGB IX zu den für die einzelnen Leistungsgesetze geltenden Vorschriften ist $\$ 7$ SGB IX. Gem $\$ 7$ SGB IX gelten die Vorschriften des SGB IX für die Leistungen zur Teilhabe nur, soweit sich aus den für den jeweiligen Rehabilitationsträger geltenden Leistungsgesetzen nichts Abweichendes ergibt (= Satz 1). Die Zuständigkeit und die Voraussetzungen für die Leistungen zur Teilhabe richten sich nach den für den jeweiligen Rehabilitationsträger geltenden Leistungsgesetzen (= Satz 2). Daraus ist zunächst abzuleiten, dass das SGB IX für sich allein keine Leistungsansprüche begründet. In der Literatur wird aber teilweise die Auffassung vertreten, dass dies nicht iS eines generellen Vorranges der Leistungsgesetze als speziellere Gesetze zu ver- 
stehen sei. Vielmehr sei das SGB IX durchaus als ein Gesetz zu interpretieren das iS einer Konkretisierung die in den Leistungsgesetzen enthaltenen Ansprüch inhaltlich beeinflusse (vgl Welti, Systematische Stellung des SGB IX im Sozialgesetzbuch - Zusammenarbeit der Leistungsträger und Koordinierung der Leistungen in SGB 08, 324).

\section{Leistungsrechtliche Probleme in der Krankenversicherung}

3.1 Das richtige Verständnis des $\$ 7$ SGB IX möchte ich zunächst anhand eines Falles aus dem Bereich der KV, der vom Bayer LSG (Urteil 23. 3. 2006, L 4 KR 279/04) entschieden wurde, näher erläutern.

In dem Rechtsstreit ging es inhaltlich um die Frage, ob die Krankenkasse eine Legasthenie-Behandlung durch ein nichtärztliches Institut durch pädagogische Lehrkräfte zu erstatten hat. Das Bayer LSG ging in der Entscheidung zunächst davon aus, dass die Legasthenie keine Krankheit iSd \$27 SGB V ist. Das Bayer LSG konnte sich hierbei sowohl auf die Rechtssprechung des BSG (vgl Urteil 25. 7. 1979, 3 RK 45/78 - SozR $2200 \$ 182 \mathrm{Nr} 48$ ) als auch des BVerwG (5. 7. 1995, 5 B 119 / 94, Buchholz 436.0 \$ 39 BSHG Nr 12) stützen. Die durchgeführten lese- und rechtschreiborientierten Fördermaßnahmen würden auch keinerlei Bezug zu einer ärztlich gebotenen oder überwachten Tätigkeit aufweisen. Eine Krankheit iSd GKV liege aber nur bei einem regelwidrigen, vom Leitbild des gesunden Menschen abweichenden Körper- oder Geisteszustand vor, der ärztlicher Behandlung bedürfe oder den Betroffenen arbeitsunfähig mache. Zudem verwies das Bayer LSG auf die Heil- und Hilfsmittelrichtlinien des ge meinsamen Bundesausschusses der Ärzte und Krankenkassen über die Verordnung in der vertragsärztlichen Versorgung, die die Übernahme von Kosten für die Behandlung der Legasthenie ausschließt. Andererseits aber sah das Gerich die Legasthenie als Behinderung iSd $\$ 11 \mathrm{Abs} 2 \mathrm{~S} 1$ SGB V an und auch die durchgeführten Behandlungsmaßnahmen der Legasthenie würden pädagogische und psychologische Hilfen im Sinne von \$26 SGB IX darstellen, die Bestandteil der medizinischen Rehabilitation sein könnten. $\$ 26$ SGB IX sei jedoch vor dem Hintergrund des $\$ 7$ SGB IX zu sehen, wo es unter S 2 SGB IX heiße, dass die Zuständigkeit und die Voraussetzungen für die Leistungen zur Teilhabe sich nach den für den jeweiligen Leistungsträger geltenden Leistungsgesetzen richten. Für jeden Träger gelte damit vorrangig das für ihn geltende Spezialgesetz vor den allgemeinen Regeln des SGB IX. Der Leistungsumfang des SGB V werde durch das SGB IX nicht erweitert (Hinweis auf BSG 26. 3. 2003 = BSGE 91, 60, 63). Im gesamten SGB V fehle es an einer Regelung, wonach eine derartige nicht ärztliche Behandlung zu erbringen wäre. Die Entscheidungsgründe des Bayer LSG lassen erkennen, dass der Senat sehr mit sich gerungen hat und sich die Entscheidung nicht leicht gemacht hat. Zur Klärung des Verhältnisses des SGB IX zum SGB V hatte seinerzeit das Bayer LSG die Revision zum BSG zugelassen, die aber nicht eingelegt wurde.

3.2 Die Entscheidung des Bayer LSG ist allerdings inzwischen durch eine neuere Entscheidung des BSG (26. 6. 2007, B 1 KR 36/06 R in Breithaupt 2009, $70-78$ ) bestätigt worden. Der Entscheidung lag folgender Sachverhalt zugrunde:
Die bei der beklagten Krankenkasse versicherte, 1981 geborene Kl leidet an einer Suchtkrankheit. Die Krankenkasse gewährte ihr eine sechsmonatige medizinische Rehabilitationsmaßnahme zur Entwöhnungsbehandlung. Noch während diese Maßnahme stellten die behandelnden Ärzte für die Versicherte bei der beklagten Krankenkasse den Antrag, der Versicherten im Anschluss an die Entwöhnungsbehandlung eine 16-wöchige Adaptionsmaßnahme zur Stabilisierung der Abstinenzfähigkeit sowie zur sozialen und beruflichen Orientierung zu gewähren. Erst nach erfolgreichem Abschluss einer entsprechenden Adaptionsmaßnahme se davon auszugehen, dass die Versicherte wieder vollschichtig leistungsfähig und voll beruflich einsetzbar sei. Die Krankenkasse hielt sich nicht für zuständig und leitete den Antrag an den klagenden Landkreis weiter und wies auf dessen Zuständigkeit im Rahmen der Sozial- und Jugendhilfe hin. Der überörtliche Träger der Sozialhilfe (Landesamt für Soziales, Jugend und Versorgung des Landes Rheinland-Pfalz) bewilligte der Versicherten die Adaptionsmaßnahme, führte aber aus, dass er als „Zweitangegangener Rehabilitationsträger iSv \$ 14 SGB IX“ handle und die Maßnahme als „Leistung der medizinischen Rehabilitation nach $\$ \$ 26$ Abs 2 , 3 SGB IX iVm $\$ 40$ Abs $1 \mathrm{~S} 1 \mathrm{Nr} 1$ Bundessozialhilfegesetz (BSHG)“ erbringe.

Vorliegend sollen nicht die spezifischen Probleme der geltend gemachten Kostenerstattung zwischen dem Landkreis und der Krankenkasse erörtert werden, sondern allein die Frage der „materiellen“ Zuständigkeit für die Adaptionsmaßnahme.

Das BSG (Urteil 26. 6. 2007, B 1 KR 36 / 06 R) hat hierzu entschieden, dass sich eine Zuständigkeit der Krankenkasse für die durchgeführte Adaptionsmaßnahme nicht daraus ergebe, dass diese Maßnahme unter den Katalog derjenigen Maßnahmen falle, die nach dem SGB IX (insb $\$ 26$ SGB IX) im Rahmen medizinischer Rehabilitation als „Haupt“- oder „Neben“-Leistungen in Betrach kommen.

In Übereinstimmung mit der Entscheidung des 3.Senates des BSG vom 26. 3.2003 (B 3 KR $23 / 02 \mathrm{R}=$ SozR $4-2500 \$ 33 \mathrm{Nr} 3 \mathrm{Rdnr}$ 15) besteht die Aufgabe der GKV auch nach Inkrafttreten des SGB IX allein in der medizinischen Rehabilitation nach Maßgabe des SGB V, also der möglichst weitgehenden Wiederherstellung der Gesundheit und der Organfunktion einschließlich der Sicherung des Behandlungserfolges, um ein selbständiges Leben führen und die Anforderungen des Alltags meistern zu können. Eine darüber hinausgehende berufliche und soziale Rehabilitation bleibt Aufgabe anderer Sozialleistungssysteme. Hinsichtlich der Zuständigkeit und der Voraussetzungen für die Leistungen zur Teilhabe wird insoweit nach wie vor auf die für den jeweiligen Leistungsträger geltenden Leistungsgesetze verwiesen, während die Vorschriften des SGB IX nur maßgebend sind, soweit etwa das SGB V nichts Abweichendes vorgesehen hat ( $\$ 7$ SGB IX). Die Krankenkassen - gem $\$ 5$ Nr $1, \$ 6$ Abs 1 Nr 1 SGB IX mögliche Träger von Leistungen zur medizinischen Rehabilitation - sind vielmehr nach den Vorschriften des SGB V zur Erbringung medizinischer Rehabilitationsleistungen nur unter den dort genannten Voraussetzungen verpflichtet. Diese Voraussetzungen ergeben sich aus den $\$ \$ 40 \mathrm{ff}, 11$ Abs 2 SGB V, wobei die in $\$ 107$ Abs 2 SGB V genannten inhaltlichen Anforderungen einzubeziehen sind (Definition der Vorsorge- und Rehabilitationseinrichtungen iSd Gesetzes). 
Aus den Feststellungen des LSG ergab sich nach Auffassung des BSG aber dass es in erster Linie um berufliche und soziale Integration der Versicherten ging, bei deren erfolgreicher Durchführung mittelbar auch eine Festigung der Erfolge der bereits vorher auf Kosten der Beklagten durchgeführten medizinischen Rehabilitation (Entwöhnungsbehandlung) zu erwarten war. Dabei fällt vor allem ins Gewicht, dass die Versicherte vor Beginn der Adaptionsmaßnahme bereits in einer anderen Rehabilitationseinrichtung eine eigenständige Maßnahme zur Entwöhnungsbehandlung erhalten hatte, die planmäßig mit der Entlassung der Versicherten als „arbeitsfähig“ endete. Es gab keine Anhaltspunkte dafür, dass diese von der Beklagten als medizinische Rehabilitationsleistung erbrachte Maßnahme ihr (medizinisches) Ziel nicht erreicht hatte und es im unmittelbaren Anschluss hieran (zB wegen Scheiterns der Entwöhnungsbehandlung) sofort einer weiteren medizinischen Rehabilitationsmaßnahme iSv $\$ 40$ SGB V bedurft hätte wie es $\$ 40$ Abs 3 Satz 2 SGB V fordert.

Der Entscheidung des BSG ist im Ergebnis zuzustimmen, wenngleich nicht hinreichend klar wird, aus welchem Satz des $\$ 7$ SGB IX es seine Schlüsse bezieht.

Der in Satz 1 des $\$ 7$ SGB X enthaltene Vorrang für die Leistungen zur Teilhabe bezieht sich auf die für den jeweiligen Rehabilitationsträger geltenden „Leistungsgesetze “. Der Begriff der Leistungsgesetze wird hierbei nicht näher definiert Unter Zugrundelegung der Gesetzesmaterialien (BT-DS 14/5074 S 100) wird man unter dem Begriff "Leistungsgesetze“ alle Bücher des Sozialgesetzbuches verstehen können, in denen Ansprüche auf Sozialleistungen geregelt sind, also insb die SGB II, III, V, VI, VII, VIII, XII. Bei diesem Verständnis erstreckt sich der Begriff des Leistungsgesetzes auf den gesamten Regelungszusammenhang, dies bedeutet sowohl auf das Leistungsspektrum als solches wie auch die Grundsätze der Ausführung der Leistungen. Der Begriff der Leistungsgesetze wird in S 2 des $\$ 7$ SGB IX wiederholt, indem sich die Zuständigkeit und die Voraussetzungen für die Leistungen zur Teilhabe nach den für den jeweiligen Rehabilitationsträger geltenden Leistungsgesetzen richten.

Für das vorliegend behandelte Verhältnis des SGB IX zur gesetzlichen KV erstreckt sich der Vorbehalt des $\$ 7$ SGB IX auch auf das Leistungserbringungsrecht ( $\$ 69 \mathrm{ff}$ SGB V), weshalb das BSG zur Begründung seiner Entscheidung zu Recht auch auf $\$ 107$ Abs 2 SGB V abstellt. Der in $\$ 7$ Satz 1 SGB IX enthaltene Vorbehalt wird in $\$ 11$ Abs 2 Satz 3 SGB V ausdrücklich wiederholt, um klarzustellen, dass aus den Regelungen des SGB IX grundsätzlich keine weitergehenden Ansprüche hergeleitet werden können.

Den beiden dargestellten Entscheidungen des Bayer LSG und BSG ist daher zuzustimmen.

Allgemein gesprochen kann gesagt werden, dass das SGB IX kein spezielles Sozialleistungsgesetz für die Rehabilitation und Teilhabe behinderter Menschen ist und ein Anspruch auf Rehabilitationsleistungen nur zusteht, wenn dieser Anspruch in dem speziellen Leistungsgesetz auch tatsächlich enthalten ist.

3.3 Mit einem weiteren ständigen Streitthema befasst sich eine andere aktuelle Entscheidung des BSG vom 19.4. 2007, B 3 KR 9 / 06 (= SozR 4 - 2500
$\$ 33 \mathrm{Nr} 15)$, nämlich mit der Frage, für welche behinderungsbedingten Folgen die gesetzliche KV unter dem Gesichtspunkt „Hilfsmittel“ einzustehen hat, weil die Folgen der Behinderung die elementaren Grundbedürfnisse des Menschen betreffen.

In dem zugrundeliegenden Fall leidet der 1947 geborene $\mathrm{Kl}$ an Multipler Sklerose (MS) und ist deswegen gehunfähig. Er bezieht Leistungen der Pflegeversicherung nach der Pflegestufe III und ist von der beklagten Krankenkasse mit einem elektrischen Rollstuhl, einem Stehrollstuhl und einer elektrischen Ladehilfe versorgt worden, mit der er den Rollstuhl bislang in den Kofferraum seines PKW verladen konnte. Er beantragt bei der Krankenkasse die Kostenübernahme für einen behinderungsgerechten Umbau seines PKW, weil es ihm wegen der Verschlimmerung seines Leidens nicht mehr möglich sei, sich vom Rollstuhl in den PKW umzusetzen und er deshalb auch die elektrische Ladehilfe nicht mehr benutzen könne. Um am gesellschaftlichen Leben - zB durch Besuch einer Selbsthilfegruppe - teilzunehmen und den Besuch von Krankengymnasten und Ärzten zu ermöglichen, müsse er nun im Rollstuhl sitzend transportiert werden.

Die Krankenkasse lehnte die Kostenübernahme - es geht um $€ 8.000,--a b$, weil es sich um eine Maßnahme zur Eingliederung in das berufliche und soziale Leben handle und die GKV hierfür nicht zuständig sei; zudem sei der Kl mit dem ihm zur Verfügung stehenden Hilfsmitteln ausreichend versorgt.

Die zu entscheidende Rechtsfrage besteht darin, ob die vom $\mathrm{Kl}$ geltend gemachten Bedürfnisse zu den Grundbedürfnissen gezählt werden, bei deren Befriedigung die Behinderung durch Hilfsmittel der medizinischen Rehabilitation auszugleichen ist ( $\$ 31 \mathrm{Abs} 1 \mathrm{Nr} 3$ SGB IX). Nach $\$ 33$ Abs $1 \mathrm{~S} 1$, 3. Alternative SGB V haben Versicherte Anspruch auf Hilfsmittel, die im Einzelfall erforderlich sind, eine Behinderung auszugleichen.

Gegenstand des Behinderungsausgleiches sind zunächst solche Hilfsmittel, die auf den Ausgleich der Behinderung selbst gerichtet sind und zum unmittelbaren Ersatz der ausgefallenen Funktionen dienen (zB Beinprothese). Der Hilfsmittelbegriff umfasst aber auch solche Hilfsmittel, die die direkten und indirekten Folgen der Behinderung ausgleichen. Ein Hilfsmittel ist von der GKV immer dann zu gewähren, wenn es die Auswirkungen der Behinderung im täglichen Leben beseitigt oder mildert und damit ein Grundbedürfnis betrifft. Nach ständiger Rsp (vgl BSGE 93, $176=$ SozR 4 - 2500 \$33 Nr 7 Rdnr 12) gehören zu den Grundbedürfnissen des täglichen Lebens das Gehen, Stehen, Greifen, Sehen, Hören, Nahrungsaufnehmen, Ausscheiden, elementare Körperpflege, selbständiges Wohnen sowie Erschließen eines gewissen körperlichen und geistigen Freiraumes.

Insoweit erkennt das BSG als Grundbedürfnis auch das Bedürfnis an, bei Krankheit und Behinderung Ärzte und Therapeuten aufzusuchen.

Allerdings begrenzt es dieses Grundbedürfnis auf den Nachbereich. Der Nahbereich wird mit der Fähigkeit umschrieben, sich in der eigenen Wohnung zu bewegen und die Wohnung zu verlassen, um die Stellen zu erreichen, an denen Alltagsgeschäfte zu erledigen sind, wobei hier ein Radius zugrundegelegt wird, den ein Gesunder zu Fuß zurücklegt. Dieser so definierte Nahbereich wird bei gehbehinderten Menschen regelmäßig durch einen handbetriebenen oder Elektro-Rollstuhl erschlossen. Dazu sei der $\mathrm{Kl}$ in der Lage. 
Im Übrigen seien nicht die konkreten Wohn- und Lebensverhältnisse eines einzelnen Versicherten maßgebend, sondern die Tatsache, dass in einem städtischen Nahbereich grundsätzlich Ärzte, Apotheken und Therapeuten vorhanden und erreichbar sind.

Das Urteil des BSG, das die zurückhaltende Linie des BSG zum Anspruch auf Hilfsmittel zum Behinderungsausgleich (zB BSG 6. 8. 1998, SozR 3 - 2500 $\$ 33 \mathrm{Nr} 29$; BSG vom 26. 3. 2003 , SozR $4-2500 \$ 33 \mathrm{Nr} 3$ ) fortsetzt, ist kritikwürdig.

Die Ansicht des BSG, es komme nur bei der Beurteilung der Erforderlichkeit iSd $\$ 33$ Abs 1 SGB V, nicht jedoch bei der Konkretisierung des Leistungsanspruches auf ein Hilfsmittel auf die Umstände des Einzelfalles an, findet im Gesetz keine Stütze. Im Gegenteil ergibt sich aus $\$ 33$ Satz 1 SGB I sogar, dass der Inhalt von Rechten nach dem SGB nach den persönlichen Verhältnissen des Beteiligten, seinem Bedarf und seiner Leistungsfähigkeit sowie den örtlichen Verhältnissen auszugestalten ist, soweit Rechtsvorschriften nicht entgegenstehen Die Verwaltung (Krankenkasse) und Gerichte hätten daher im konkreten Einzelfall untersuchen müssen, ob das Grundbedürfnis der medizinischen Versorgung im Nahbereich erfüllt werden kann. Die abstrakte Annahme, an jedem Ort der BRD könne mit einem Elektrorollstuhl die nötige medizinische Versorgung erreicht werden, ist jedenfalls lebensfremd. Bei der Prüfung hätte im Übrigen auch die freie Arztwahl berücksichtigt werden müssen. Die freie Arztwahl nach $\$ 76$ Abs 1 SGB V im Rahmen der vertragsärztlichen Versorgung ist Ausdruck der gewünschten Patientensouveränität und der autonomen Selbstbestimmung. Diese Selbstbestimmung ist aber gerade nach $\S 10$ SGB I und $\$ \$ 1,4$ Abs 1 Nr 4 SGB IX ein wesentliches Ziel der Leistungen für behinderte Menschen.

Fragwürdig ist auch die Auffassung des BSG, der Bèsuch einer Selbsthilfegruppe zähle nicht zu den Grundbedürfnissen. ME ist die Kooperation mit Gleichbetroffenen ein menschliches Grundbedürfnis, das als wichtiger Bestandteil einer selbständigen und selbstbestimmten Lebensführung iS von $\$ 4 \mathrm{Abs} 1$ $\mathrm{Nr} 4$ SGB IX gelten kann.

Es ist insgesamt zu bezweifeln, ob das starre Abstellen auf den sog „Nahbereich" insgesamt geeignet ist, Grundbedürfnisse von weiteren Bedürfnissen unter dem Blickwinkel Mobilität behinderter Menschen abzugrenzen (vgl Welti in „Diskussionsbeiträge zu Leistungen zur Teilhabe und Rehabilitation“, iqpr Köln 2008, $195 \mathrm{f}$ ).

\section{Leistungsrechtliche Probleme in der Rentenversicherung}

Das dargestellte Verhältnis zwischen SGB IX und den einzelnen Leistungsgesetzen gilt in gleicher Weise im Bereich der Rentenversicherung - SGB VI Das SGB IX begründet auch hier allein keine Leistungsansprüche. Auch hier ist vielmehr der Rückgriff auf das fachspezifische Leistungsgesetz erforderlich. Im Falle der Rentenversicherung sind dies die $\$ 9-33$ SGB VI. Hier lohnt sich ein Blick auf $\$ 9$ SGB VI. Dort heißt es in Abs 1, Satz 1 unter anderem: „Die Rentenversicherung erbringt Leistungen zur medizinischen Rehabilitation". Gem Abs 2 können die Leistungen nach Abs 1 erbracht werden, wenn die persönlichen und versicherungsrechtlichen Voraussetzungen hierfür erfüllt sind. Das Ermessen be- zieht sich dabei nicht auf das „Ob“ der Leistungserbringung, sondern nur auf das „Wie“. Hierbei ist gegebenenfalls auch das Wunsch- und Wahlrecht des $\$ 9$ SGB IX zu beachten, auf das ich an dieser Stelle näher eingehen möchte.

Das Wunsch- und Wahlrecht des $\$ 9$ SGB IX wurde mit dem SGB IX neu neben der bis dahin allein geltenden Vorschrift des $\$ 33$ SGB I zum 1.7.2001 eingeführt. Es verpflichtet die Rehabilitationsträger, sowohl bei der Entscheidung als auch bei der Ausführung der Leistungen zur Teilhabe berechtigten Wünschen der Leistungsberechtigten zu entsprechen und dabei auf die persönliche Lebenssituation, das Alter, das Geschlecht, die Familie sowie die religiösen und weltanschaulichen Bedürfnisse des Leistungsberechtigten Rücksicht zu nehmen.

Nach $\$ 33$ SGB I hatten die Rehabilitationsträger schon bisher bei der Ausgestaltung von nach Art und Umfang inhaltlich im Einzelnen nicht bestimmten Rechten und Pflichten die persönlichen Verhältnisse der Berechtigten, seinen Bedarf, seine Leistungsfähigkeit und seine örtlichen Verhältnisse zu berücksichtigen und angemessenen Wünschen zu entsprechen.

$\$ 9$ SGB IX konkretisiert diese allgemeine Verpflichtung für den Bereich der Leistungen zur Teilhabe. Daran ändert auch die in $\$ 9$ Abs 1 Satz 2, 2 HS SGB IX enthaltene Verweisung ,im Übrigen gilt $\$ 33$ des Ersten Buches" nichts. Der in $\$ 33$ SGB I enthaltene ebenfalls unbestimmte Rechtsbegriff ,angemessen“ spielt bei der Auslegung des Begriffes "berechtigt" keine Rolle. Bei der undifferenzierten Verweisung in \$9 Abs 1 S 2, 2 HS SGB IX handelt es sich zumindest um ein Redaktionsversehen des Gesetzgebers, soweit man nicht ohnehin davon ausgeht, dass $\$ 9$ Abs 1 SGB IX („berechtigte Wünsche") als lex specialis den „angemessenen Wünschen" in $\$ 33$ SGB I vorgeht. Keinesfalls kann es jedenfalls sein, dass als berechtigt anzuerkennende Wünsche iSv $\$ 9$ Abs 1 S 2 HS 2 SGB IX einer weitergehenden Angemessenheitsprüfung seitens der Sozialverwaltung unterzogen werden könnten. Während der Begriff des angemessenen Wunsches in $\$ 33$ S 2 SGB I eine Abwägung zwischen mehreren Alternativen enthält, ist ein berechtigter Wunsch ein solcher, dem keine Rechtsvorschrift entgegensteht.

Bei den in $\$ 9$ Abs 1 Satz 1 SGB IX genannten „Wünschen“ handelt es sich um einen unbestimmten Rechtsbegriff. Von den Wünschen werden alle von dem Leistungsberechtigten geäußerten Willensbekundungen hinsichtlich der in Frage stehenden Leistung zur Teilhabe umfasst. Wobei die geäußerten Wünsche nur dann als "berechtigt" angesehen werden können, wenn sie sich im Rahmen des allgemeinen Leistungsrechts halten. Das Wunschrecht kann nicht ein „Mehr" an Leistungen erbringen, als in der Anspruchsnorm vorgesehen ist. Leistungsausschlüsse, Lücken im Gesetz, Zuzahlungsregelungen können mit dem Wunschrecht nicht überwunden werden.

Ein typischer, im Zusammenhang mit dem Wahl- und Wunschrecht gem $\$ 9$ SGB IX auftretender Streitfall ergibt sich aus einem von LSG Rheinland-Pfalz entschiedenen Fall.

Fall:

Die 1954 geborene Kl befindet sich wegen ihrer Alkoholabhängigkeit stationär in einer Klinik. Unter Bezugnahme auf eine Bescheinigung ihrer behandelnden Ärzte beantragte sie die Gewährung medizinischer Rehabilitationsleistungen 
für Alkoholabhängige gem $\$ 15 \mathrm{SGB}$ VI, wobei das Wahl- und Wunschrecht gemäß $\$ 9$ SGB IX ausgeübt und als Behandlungsstätte eine Fachklinik für Frauen ausgewählt wurde, mit der der Rentenversicherungsträger keinen Vertrag gem $\$ 21$ SGB IX abgeschlossen hatte.

Der Rentenversicherungsträger ist dem Wunsche der Kl insoweit nicht gefolgt, als zwar eine stationäre medizinische Leistung bewilligt wurde, aber nich in der gewünschten Fachklinik für suchtkranke Frauen, sondern in der eigenen Fachklinik.

Das LSG Rheinland-Pfalz hat die Entscheidung des Rentenversicherungsträgers bestätigt mit der Begründung, dass ein Wunsch iSd \$9 SGB IX nich berechtigt ist, wenn der behinderte Mensch Leistungen in einer Einrichtung erhalten möchte, die nicht vom Rehabilitationsträger betrieben wird und mit welcher dieser keinen Vertrag geschlossen hat. Dass außerhalb solcher Einrichtungen eine Rehabilitation nicht in Betracht kommt, folge auch aus $\$ 15$ Abs II $S 1$ SGB VI. Daran ändere auch das Wunsch- und Wahlrecht des Versicherten gem $\$ 9$ SGB IX nichts. Der Entscheidung ist meines Erachtens beizupflichten. Entgegen der teilweise in der Literatur (vgl Welti, SGB 2003, 379, 385) geäuBerten Auffassung ist der Rentenversicherungsträger auch nicht verpflichtet, mit einer bestimmten Klinik wegen des Wunsch- und Wahlrechtes gem $\$ 9$ SGB IX einen Vertrag abzuschließen. Das SGB IX konkretisiert zwar die im SGB VI enthaltenen Anforderungen an Einrichtungen (vgl $\$ 15$ Abs 2 SGB VI), indem es den Vertragsschließenden inhaltliche Vorgaben macht ( $\$ 21$ Abs $1 \mathrm{Nr} 1$ bis 6 , 35 Abs 1 S 2 Nr 1 bis 4 SGB IX) und das Abschluss- sowie Auswahlermessen des Rentenversicherungsträgers an gesetzliche Ziele bindet ( $\$ 19$ Abs 4 SGB IX). Die Verpflichtung des Rentenversicherungsträgers zum Vertragsabschluss ergibt sich daraus aber nicht. Aus der Gesetzesbegründung zum Wünsch- und Wahlrecht gem $\$ 9$ SGB IX (abgedruckt bei Hauck/Noftz, SGB IX M 010, 191) geht eindeutig hervor, dass ein Wunsch nur dann berechtigt ist, wenn er sich ua im Rahmen des Leistungsrechts und sonstiger Vorgaben hält, hierzu zählt auch die Vorgabe, dass Leistungen nur in Einrichtungen zu erbringen sind, in denen ein Vertrag nach $\$ 21$ SGB IX besteht.

\section{Leistungsrechtliche Probleme in der UV}

Wesentlich komfortabler stellt sich die Rechtslage für den Versicherten bei der medizinischen Rehabilitation in der UV dar.

In der gesetzlichen UV ist die medizinische Rehabilitation Bestandteil der Heilbehandlung. Die gesetzliche UV ist von dem Grundsatz geprägt, dass die Rehabilitation mit allen geeigneten Mitteln - so die Formulierung in $\$ 26$ Abs 2 SGB VII - zu leisten ist.

Daraus folgt, dass der Leistungsrahmen für die Rehabilitation im Rahmen der UV weiter ist als in anderen Zweigen der SV, etwa in der KV, wo die Leistungen auf eine ausreichende, zweckmäßige und wirtschaftliche Versorgung ausgerichtet sind und das Maß des Notwendigen nicht überschreiten dürfen (vgl $\$ 12$ Abs 1 SGB V).

Dies bedeutet zunächst, dass einerseits kein Kosten-Nutzen-Vergleich stattfindet und deswegen auch außergewöhnlich hohe finanzielle Aufwendungen in
Kauf zu nehmen sind, wenn das Ziel der Rehabilitation nur auf diesem Wege zu erreichen ist. Die UV-Träger können sogar Leistungen erbringen, die in dem Leistungskatalog der $\$ \$ 28 \mathrm{ff}$ SGB VII nicht enthalten sind.

Ein anschauliches Beispiel für die Rechtslage der medizinischen Rehabilitation in der UV ergibt sich aus einem vom LSG Rheinland-Pfalz Urteil vom 11. 10. 2005, L 3 U 273 / 04 entschiedenen Fall.

Fall:

Die Kl erlitt bei der Tätigkeit in der Landwirtschaft einen Arbeitsunfall, für den der beklagte UV-Träger zuständig ist. Bei dem Unfall kam es bei der Kl zur Zerquetschung und Zertrümmerung des gesamten linken Unterschenkels und des Fußes mit Eröffnung des Kniegelenks und Bruch im Bereich der Condylen des Oberschenkels. Der Oberschenkel wurde im mittleren Drittel amputiert und prothetisch versorgt.

Die Berufsgenossenschaft sah bei der $\mathrm{Kl}$, weil sie außerordentlich sensibel und im Gangbild vorsichtig und unsicher war, die Indikation für die Versorgung mit einer Prothese mit einem mikroprozessorgesteuerten Kniegelenk (C-Leg) trotz der enormen Kosten für gegeben an. Bereits zwei Jahre später beantragte die Kl die Gewährung einer technischen Weiterentwicklung, einen Kunstfuß Luxon-Max.

Das von der Berufsgenossenschaft eingeholte Gutachten bestätigte, dass der von der Kl begehrte Kunstfuß einen Teil der Stöße während der Auftrittsphase abfangen könne, woraus sich eine Entlastung der Hüfte ergebe. Jede technische Neuentwicklung bedeute eine Verbesserung der bisherigen technischen Möglichkeiten. Die Berufsgenossenschaft hat die Kostenübernahme für den Kunstfuß Luxon-Max gleichwohl abgelehnt. Bei der Ausübung des Ermessens, welche Rechtsfolge gemäß $\$ 26$ Abs 5 SGB VII gesetzt werden solle (Auswahlermessen) sei einerseits das Rehabilitationsziel, andererseits aber auch der Grundsatz der Wirtschaftlichkeit und Sparsamkeit zu beachten. Das Sozialgericht Trier und das LSG Rheinland-Pfalz sind dem nicht gefolgt und haben der Kl einen Anspruch auf den begehrten Kunstfuß Luxon-Max zugesprochen. Dem ist zu folgen.

Im UV-Recht hat der Versicherte einen Anspruch auf Heilbehandlung, wobei der UV-Träger "mit allen geeigneten Mitteln" den durch den Versicherungsfall verursachten Gesundheitsschaden zu beseitigen oder zu bessern, seine Verschlimmerung zu verhüten und seine Folgen zu mindern hat. Bei der im Einzelfall zu treffenden Entscheidung des UV-Trägers handelt es sich um eine Entscheidung nach pflichtgemäßem Ermessen über Art und Umfang der geschuldeten Leistung. Dieses Ermessen kann durch die gesetzliche Prämisse „mit allen geeigneten Mitteln“ auf Null reduziert sein, wenn es nur ein geeignetes Mittel gibt und aus der Wertentscheidung heraus folgt, dass das geeignete Mittel einzusetzen ist. Der UV-Träger hat vorliegend verkannt, dass es nicht um eine ,ausreichende“ Versorgung des Versicherten geht. Wegen des umfassenden Heilbehandlungsauftrages des UV-Trägers sind die Maßnahmen anders als im Bereich der gesetzlichen $\mathrm{KV}$, in deren Rahmen eine notwendige und ausreichende Leistung zu gewähren ist ( $\mathrm{vgl} \S 12$ SGB V), nicht entsprechend begrenzt. 
Für die Frage der Versorgung mit Heil- und Hilfsmitteln gilt nach dem Recht der gesetzlichen UV nicht der Grundsatz einer wirtschaftlichen, sondern einer optimalen Rehabilitation. Die dabei zu beachtende Grenze ist allein die Geeignetheit.

\section{Geld- und Sozialleistungen}

Für das Rehabilitationsrecht gilt - wie auch sonst im SV-Recht bzw Sozialrecht - das Sachleistungsprinzip. Danach sind die Leistungen der Rehabilitation in Natura zu gewähren. Sach- und Dienstleistungen stehen allen Anspruchsberechtigten in gleicher Höhe und Dauer unabhängig von der Höhe der zuvor geleisteten Beiträge zu. Daran hat das zum 1.7.2001 in Kraft getretene SGB IX nichts geändert.

6.1 Mit dem zum 1. 7. 2001 in Kraft getretenen SGB IX ist allerdings speziell für das Rehabilitationsrecht in $\$ 15$ SGB IX eine Regelung über die Kostenerstattung selbstbeschaffter Leistungen geschaffen worden.

Vor Inkrafttreten des SGB IX enthielt lediglich $\$ 13$ Abs 3 SGB V aF ( GKV) für den Bereich der Krankenbehandlung eine Regelung, die von der höchstrichterlichen Rechtsprechung im Bereich der Rehabilitation entsprechend angewandt wurde.

$\$ 15$ SGB IX unterscheidet zwei Fallvarianten der Kostenerstattung, nämlich die Kostenerstattung nach Fristsetzung und die Kostenerstattung bei einer unaufschiebbaren Leistung oder einer rechtswidrigen Ablehnung. Die Voraussetzungen der neu geregelten Kostenerstattung nach Fristsetzung ergeben sich aus $\$ 15$ Abs 1 S 1 - 3 SGB IX. Danach muss der Rehabilitationsträger, wenn er über einen Antrag auf Leistungen zur Teilhabe nicht innerhalb der in $\$ 14$ Abs 2 genannten Frist ( $=3$ Wochen!) entscheiden kann, dies dem Leistungsberechtigten unter Darlegung der Gründe rechtzeitig mitteilen. Erfolgt diese Mitteilung nich oder ist der dargelegte Grund nicht „zureichend“, können Leistungsberechtigie dem Rehabilitationsträger eine „angemessene“ Frist setzen und erklären, dass sie nach Ablauf der Frist die erforderliche Leistung selbst beschaffen werden $(\$ 15$ Abs 1 S 2 SGB IX). Beschaffen sich Leistungsberechtigte nach Ablauf der Frist eine erforderliche Leistung selbst, ist der zuständige Rehabilitationsträger unter Beachtung der Grundsätze der Wirtschaftlichkeit und Sparsamkeit zur Erstattung der Aufwendungen verpflichtet.

Die unbestimmten Rechtsbegriffe "zureichender Grund" und "angemessen“ werden dabei im Gesetz oder in der Gesetzesbegründung nicht näher erläutert. Ein „zureichender Grund“ liegt immer dann vor, wenn der Rehabilitationsträger die Verzögerung nicht zu vertreten hat, wenn also bei objektiver Würdigun der vorgebrachten Gründe diese als ausreichende Hindernisse anzusehen sind und nicht der Einflusssphäre des Rehabilitationsträgers zuzuordnen sind. Als "angemessene" Frist ist nach allgemeiner Meinung eine Frist von wenigstens 2 Wochen - bzw drei weiteren Wochen bei Notwendigkeit der Einholung eines Gutachtens - ausreichend. Diese letztlich dem Gesetz zu entnehmenden Fristen stellen den Rehabilitationsträger schon allein rein zeitlich vor gewaltige Herausforderungen.
Beschafft sich der Leistungsberechtigte die erforderliche Rehabilitationsleistung nach Ablauf der angemessenen Frist selbst, ist der Rehabilitationsträger zur Erstattung der Aufwendungen unter Beachtung der Grundsätze der Wirtschaftlichkeit und Sparsamkeit verpflichtet. Dies heißt aber nicht notwendiger Weise, dass nur diejenigen Kosten zu erstatten wären, die der Rehabilitationsträger bei eigener rechtzeitiger Leistungserbringung hätte aufwenden müssen (so aber Benz NZS, 2002, 511, 513). Natürlich kann der Leistungsberechtigte über den Weg der Kostenerstattung nicht Kosten für eine "Luxus-Reha" dem Rehabilitationsträger aufbürden. Diejenigen Kosten, die aber gerade deswegen anfallen, weil der Leistungsberechtigte sich die Leistung selbst beschaffen muss - möglicherweise auch unter Zeitdruck - gerade weil der Rehabilitationsträger nicht tätig geworden ist, sind vom Rehabilitationsträger zu erstatten. Zu erstatten ist nach dem Gesetzeswortlaut aber immer nur die „erforderliche“ Leistung. Eine erforderliche Leistung liegt jedenfalls dann vor, wenn deren Tatbestandsvoraussetzungen im jeweiligen Leistungsgesetz erfüllt sind und die selbst beschaffte Leistung im Rahmen der Ermessungsentscheidung des Rehabilitationsträgers nicht rechtswidrig wäre (so Benz, Kostenerstattung NZS, 513; weitergehende Auffassung vgl BSGE 66, 275, 283 mit Anm Boecken, SGb 1991, 147 ff).

Insgesamt ist festzustellen, dass der Weg über die Kostenerstattung für selbst beschaffte Rehabilitationsleistungen nach Fristsetzung in der Praxis gegenüber der der schon seit längerem geltenden Regelung in $\$ 13$ Abs 3 SGB V nachgebildeten Fallvariante des $\$ 15$ Abs 1 S 4 SGB IX (Rehabilitationsträger hat eine unaufschiebbare Leistung nicht rechtzeitig erbracht oder zu Unrecht abgelehnt) deutlich seltener beschritten wird. Dies liegt wohl daran, dass letztlich der Versicherte das Risiko für das Vorliegen der unbestimmten Rechtsbegriffe „zureichender Grund“, , ,angemessen“ und „erforderlich“ trägt mit der Folge, dass er möglicherweise ganz oder zum Teil auf den verauslagten Kosten sitzen bleibt. Vor diesem tatbestandsmäßig unsicherem Hintergrund hat der Versicherungsträger meines Erachtens eine Informations- und Beratungspflicht gegenüber dem Versicherten, um diesem die mit einer Selbstbeschaffung verbundenen Risiken zu verdeutlichen, wenn der Rehabilitationsträger erkennt, dass die vom Versicherten beantragte Leistung nicht erforderlich ist oder zureichende Gründe vorliegen, die beantragte Leistung nicht innerhalb der gesetzlichen Fristen zu erbringen ( $\$ \$ 14$, 16 Abs 3 SGB I). Unterbleibt dies und hat dies zur Folge, dass der Versicherte durch selbst beschaffte Rehabilitationsleistungen wirtschaftliche Nachteile erleidet, so sind diese ggf im Wege des sozialrechtlichen Herstellungsanspruches vom Rehabilitationsträger auszugleichen. Voraussetzung ist allerdings, dass der Anspruchsteller die geforderte Leistung, die er sich selbst beschaffen will, so genau beschreibt, dass der Reha-Träger seiner Beratungs- und Informationspflicht auch nachkommen kann.

6.2 Persönliches Budget: Kann man einem Versicherten einfach Geld in die Hand geben, damit er sich selbständig und eigenverantwortlich die notwendige Rehabilitationsleistung selbst kauft?

Diese Frage wird mit dem in $\$ 17$ SGB IX ( $\$ 17$ Abs $2-5$ SGB IX) seit 1.7.2001 vorgesehenen, zunächst als Ermessungsleistung, mittlerweile ab 
1. 1.2008 als Rechtsanspruch ausgestatteten „persönliches Budget" mit einem klaren Ja beantwortet.

Mit dem persönlichen Budget hat der Gesetzgeber eine neue Variante der Leistungserbringung neben Sachleistungserbringung und Kostenerstattungsprinzip geschaffen.

Das persönliche Budget ist ein Geldbetrag, mit dem sich der Versicherte eine Rehabilitationsleistung einkaufen kann. Der Versicherte hat jetzt die Wahl: Er kann weiterhin das Gesamtpaket „Sachleistung“" oder das Selbstmanagementprogramm „Persönliches Budget“ wählen.

Oberstes Ziel des Persönlichen Budgets ist es, die Selbstbestimmung und die Eigenverantwortung des behinderten Menschen zu fördern.

Wie läuft das Verfahren nun im Einzelnen ab (vgl \$\$17 Abs 2 - 5 SGB IX)?

Eine Leistungserbringung als „Persönliches Budget" setzt zunächst immer einen Antrag des Versicherten voraus ( $\$ 17$ Abs 1 Satz 1 SGB IX). Gegen den Willen des Versicherten kann eine Leistungserbringung im Wege des persönlichen Budgets daher nicht erfolgen.

Der nach \$14 SGB IX zuständige Leistungsträger leitet dann unverzüglich ein Bedarfsfeststellungsverfahren ein. Ermittelt werden muss, welche Leistungen zur Teilhabe unter Berücksichtigung der individuellen Situation des Leistungsberechtigten sowie bei Wahrung seines Wunsch- und Wahlrechtes notwendig sind, welche Hilfen zur Deckung des Bedarfs in Betracht kommen und wie hoch die finanziellen Kosten ausfallen. Zum Bedarf gehört ausdrücklich auch die wegen der Leistungsausführung als persönliches Budget notwendig werdende Beratung und Unterstützung (Budgetassistenz). Da die Höhe der persönlichen Budgets die Kosten der zur Bedarfsdeckung erforderlichen Leistungen, wie sie bei einer anderen Form der Leistungsausführung entstehen würden, in der Regel nicht überschreiten soll, sind auch hierzu Feststellungen zu treffen. Von den dabei gefundenen Ergebnissen hängt $a b$, ob der individuelle Bedarf tatsächlich durch das persönliche Budget gedeckt werden kann. Für den Bereich der medizinischen Rehabilitation ist grundsätzlich davon auszugehen, dass alle medizinischen Rehabilitationsleistungen grundsätzlich budgetfähig sind. Ist das Bedarfsfeststellungsverfahren abgeschlossen, schließen der Leistungsträger bzw der Budgetbeauftragte eine Zielvereinbarung, in der mindestens geregelt sein müssen:

- die unter Berücksichtigung des Einzelfalles mit den Leistungen und den geplanten Fördermaßnahmen konkret angestrebten Ziele,

- ob und in welcher Form ein Nachweis geführt werden muss, dass der festgestellte Bedarf auch tatsächlich durch die Inanspruchnahme von Leistungen gedeckt werden kann,

- wie die Qualität der von dem behinderten Menschen zur Deckung seines Hilfsbedarfes in Anspruch genommenen Leistungen gesichert werden soll.

Ist eine Zielvereinbarung abgeschlossen und damit das Bedarfsfeststellungsverfahren beendet, erlässt der zuständige Leistungsträger den Bescheid, mit dem die Ausführung der Leistungen als „Persönliches Budget" bewilligt wird.

Die bisherige Praxis zeigt, dass viele behinderte Menschen und deren Angehörige auf der einen Seite, aber auch Mitarbeiter von Leistungsträgern und Leistungserbringern dem neuen Instrument „Persönliches Budget“ noch kritisch gegenüber stehen. Insbesondere viele behinderte Menschen empfinden offensichtlich das beschriebene Verfahren als aufwendig und bürokratisch. Tatsächlich bedeutet mehr Selbstbestimmung auch mehr Eigenverantwortlichkeit und dies wiederum mehr persönlichen Einsatz als bei Leistungserbringungsformen, die sozusagen standardisiert von Leistungsträgern und Leistungserbringern angeboten werden. Dieser mögliche Nachteil wird aber aufgewogen dadurch, dass der Versicherte mit Hilfe des Persönlichen Budgets Leistungen erkaufen kann, die er sonst nicht bekäme. Zwar sind die in Frage kommenden Rehabilitationsleistungen in den einzelnen Leistungsgesetzen weitgehend abschließend definiert. Jedoch können diese Leistungen im Rahmen des Persönlichen Budgets im Einzelfall passgenauer in Anspruch genommen werden. Aber auch die Verwaltung dürfte profitieren. Durch den direkten Kontakt mit dem Versicherten kommt es zu sachgerechteren Einschätzungen im konkreten Fall und damit zu wirkungsvolleren und nachhaltigeren Maßnahmepaketen.

\section{Zuzahlungsregelungen}

Bei den Leistungen zur medizinischen Rehabilitation sind zum Teil Zuzahlungen zu leisten. Einzelheiten ergeben sich $\mathrm{zB}$ für den Bereich der gesetzlichen KV aus den $\$ \$ 61,62$ SGB V und für den Bereich der gesetzlichen Rentenversicherung aus $\$ 32$ SGB VI. Probleme grundsätzlicher Natur ergeben sich bei der Anwendung dieser Vorschriften nicht.

Ein eingehenderer Blick lohnt sich aber auf die durch die Gesundheitsreform 2007 zum 1. 4. 2007 ins SGB V aufgenommene Vorschrift des $\$ 40$ Abs 2 Satz 2 SGB V. Dort heißt es: „Wählt der Versicherte eine andere zertifizierte Einrichtung, mit der kein Versorgungsvertrag nach $\$ 111$ SGB V besteht, so hat er die dadurch entstehenden Mehrkosten zu tragen“".

Grundsätzlich ist die Frage des Verhältnisses der Ausübung des Wunschund Wahlrechtes zur Frage der Tragung von Mehrkosten dahingehend zu beantworten, dass der für die stationäre medizinische Rehabilitation zuständige Sozialleistungsträger nicht nur die Pflicht zur Organisation, sondern auch - vorbehaltlich einer abweichenden gesetzlichen Regelung - die Pflicht zur vollständigen Finanzierung der Leistungserbringung entsprechend dem Sachleistungsprinzip hat. Andernfalls würde die Aufbürdung der Zahlung der Mehrkosten zu einer Konterkarierung des Wunschrechts des Leistungsberechtigten führen, indem dessen Verwirklichung an ein fiskalisches, gesetzlich nicht normiertes Amortisationsgebot gekoppelt würde. Einzige Ausnahme hierzu ist der genannte $\$ 40$ Abs 2 Satz 2 SGB V, der iSd $\$ 7$ S 1 SGB IX trägerspezifisches abweichendes Recht zum Wunschrecht des $\$ 9$ SGB IX enthält, nicht jedoch zum Leistungserbringungsrecht zu den $\$ \$ 17,19$ SGB IX.

Das damit für den Bereich der KV geschaffene trägerspezifische Recht bewirkt im Verhältnis zu dem für die übrigen Rehabilitationsträger geltenden Wunschrecht gem $\$ 9$ Abs 1 SGB IX eine Schlechterstellung des Versicherten. Im Bereich der GKV hat er deshalb etwaige mit seinem Wunsch verbundene Mehrkosten ohne weitere Prüfung immer selbst zu tragen. Alle übrigen Träger der medizinischen Rehabilitation haben dagegen bei Ausübung des Wunschrechtes unter Berücksichtigung der Rechtsprechung des BSG bei gleicher Qualität und 
Wirksamkeit der gewünschten Leistungsausführung im Verhältnis zu den Kosten der Vertragskliniken anfallende Mehrkosten zu übernehmen. Insoweit ist auch eine Auseinanderentwicklung des durch das SGB IX vereinheitlichten Rehabilitationsrechts zu beklagen.

\section{Mitwirkungsobliegenheit und Zumutbarkeit}

Gem $\$ 63$ SGB I soll sich, wer wegen Krankheit oder Behinderung Sozialleistungen beantragt oder erhält, auf Verlangen des zuständigen Leistungsträgers einer Heilbehandlung unterziehen, wenn zu erwarten ist, dass sie eine Besserung seines Gesundheitszustandes herbeiführen oder eine Verschlechterung verhindern wird.

Der in $\$ 63$ SGB I verwendete Begriff der Heilbehandlung ist dabei weit auszulegen und umfasst alle Maßnahmen, die in den Bereichen Krankenbehandlung und medizinische Rehabilitation geeignet sind, Krankheiten zu heilen, zu lindern und Behinderung bewirkende Krankheitsfolgen bestmöglich zu verringern.

Durch $\$ 63$ SGB I werden zwei Sozialleistungen miteinander verknüpft. Der Sozialleistungsträger nimmt eine beantragte oder gewährte Sozialleistung zum Anlass, auf die Inanspruchnahme einer anderen Sozialleistung hinzuwirken, damit die kostspielige beantragte Leistung ganz entbehrlich wird oder herabgesetzt werden kann. In dem Kontext „Reha vor Rente“ wird also der Anspruch auf eine Rentenleistung davon abhängig gemacht, dass vor Bewilligung einer Rentenleistung (insbesondere wegen Erwerbsminderung, $\$ 43$ SGB VI und wegen teilweise Erwerbsminderung bei Berufsunfähigkeit, \$240 SGB VI) alle Möglichkeiten eine Rehabilitation ausgeschöpft sind. In der Fallgestaltung des $\$ 63$ SGB I geht also die Initiative zur Durchführung einer medizinischen Rehabilitationsmaßnahme zunächst nicht vom Versicherten, sondern vom Sozialleistungsträger aus.

Die Pflicht des Sozialleistungsträgers hier tätig zu werden, ergibt sich allgemein aus seiner Verpflichtung, eine sachgerechte, den tatsächlichen Umständen entsprechende Entscheidung herbeizuführen, die auch beinhaltet, mit den vorhandenen (finanziellen) Mitteln zielgerichtet umzugehen.

Diese Pflicht ist durch den schon genannten Grundsatz "Reha vor Rente" sowohl spezialgesetzlich ( $\$ 9$ Abs 1 S 2 SGB VI, $\$ 26$ Abs 3 SGB VII) als auch allgemein in $\$ 8$ Abs 2 SGB IX geregelt. Der Grundsatz „Reha vor Rente“ ist aber keine selbständige Sanktionsnorm und hat für den Versicherten nur Auswirkungen im Rahmen und nach Maßgabe der $\$ 60$ ff SGB I.

$\$ 63$ SGB I verpflichtet den Leistungsberechtigten nicht nur zur Duldung der notwendigen Heilbehandlungsmaßnahmen, sondern auch dazu, bei deren Durchführung nach besten Kräften mitzuwirken. Der Einzelne hat den konkreten Anordnungen des Arztes zur Heilung nachzukommen, zB eine Diät einzuhalten, ein bestimmtes Medikament einzunehmen.

Die Aufforderung zu einer Heilbehandlung ist ein Verwaltungsakt (aA Mrozynski, SGB I $\$ 63$ Rdnr 4 wie hier Hauck/Noftz, SGB I $\$ 63$ Rdnr 10; Seewald in Kassler Kommentar, SGB I, \$ 63 Rdnr 10).

Grundsätzlich legt der zuständige Leistungsträger Art und Ort der medizinischen Rehabilitation fest. Der Leistungsträger ist aber gehalten, die für den
Versicherten bestmögliche medizinische Rehabilitationsmaßnahme in enger Kooperation und vorheriger Absprache mit diesem zu finden. Auch das schon angesprochene Wunsch- und Wahlrecht des Leistungsberechtigten gem $\$ 9$ SGB IX spielt hier eine gewichtige Rolle.

Die Pflicht "an einer medizinischen Rehabilitationsmaßnahme" mitzuwirken, hat auch ihre Grenzen. Diese sind allgemein in $\$ 65$ SGB I festgelegt, wobe die dort aufgeführten Verweigerungsgründe bei einer medizinischen Rehabilitation eher selten vorliegen dürften.

Kommt aber derjenige, der eine Sozialleistung wegen ua Gefährdung oder Minderung der Erwerbsfähigkeit beantragt oder erhält, seinen Mitwirkungspflichten nach den $\$ \$ 62-65$ SGB I nicht nach und ist unter Würdigung aller Umstände mit Wahrscheinlichkeit anzunehmen, dass deshalb die Fähigkeit zur Erwerbstätigkeit beeinträchtigt oder nicht verbessert wird, kann der Leistungsträger die Leistung bis zur Nachholung der Mitwirkung ganz oder teilweise versagen oder entziehen ( $\$ 66$ Abs 2 SGB I).

Das beschriebene Verfahren nach den $\$ \$ 60 \mathrm{ff}$ SGB I ist freilich nicht sehr praktikabel. Dies liegt daran, dass gegen die Versagung einer Sozialleistung wegen fehlender Mitwirkung in einem anschließenden Gerichtsverfahren nur die reine Anfechtungsklage gegeben ist. Streitgegenstand eines solchen Rechtsstreits ist nicht der materielle Anspruch, sondern die Auseinandersetzung über Rechte und Pflichten der Beteiligten im Verwaltungsverfahren mit dem Ziel das Verwaltungsverfahren nach Aufhebung des Versagungsbescheides fortzusetzen. Selbst ein nach erfolgter Mitwirkung ergangener Bescheid in der Sache wird nicht gem $\$ 96$ SGG Gegenstand des auf Aufhebung des Versagungsbescheides mangels Mitwirkung gerichteten Verfahrens.

\section{Entgeltfortzahlung durch den Arbeitgeber während} Maßnahmen der medizinischen Rehabilitation

Während einer Maßnahme der medizinischen Rehabilitation besteht für den Versicherten unter den Voraussetzungen des $\$ 9$ EFZG Anspruch auf Entgeltfortzahlung. Die Bestimmung des $\$ 9$ Abs 1 EFZG stellt eine medizinische Rehabilitationsmaßnahme einer Arbeitsunfähigkeit des Arbeitnehmers gleich und gewährleistet entsprechende Entgeltfortzahlungsansprüche auch falls der Arbeitnehmer in dieser Zeit nicht arbeitsunfähig erkrankt ist. Dies geschieht dadurch, dass die für die Entgeltfortzahlung bei krankheitsbedingter Arbeitsunfähigkeit geltenden Regeln $(=\$ \$ 3-4 \mathrm{a}$ und $6-8 \mathrm{EFZG})$ weitgehend für anwendbar erklärt werden.

a) Voraussetzungen des $\$ 9$ EFZG: Es muss sich um eine medizinische Rehabilitationsmaßnahme handeln, also dazu dienen, eine bestimmte Krankheit endgültig auszuheilen, die Gesundheit wiederherzustellen bzw den Organismus nach überstandener Krankheit zu kräftigen (vgl BAG, AP Nr 2 zu $\$ 7$ LFZG). Die Rehabilitationsmaßnahme muss notwendig sein. Dies steht jedoch im Regelfall fest, wenn die Maßnahme von einem öffentlich-rechtlichen Sozialleistungsträger bewilligt worden ist. Die Ermessensentscheidung durch den Sozialleistungsträger lässt sich arbeits- 
rechtlich (!) nur daraufhin überprüfen, ob ein offensichtlicher Ermessensfehlgebrauch, $\mathbf{z B}$ in Gestalt einer völligen Verkennung des Begriffs der medizinischen Rehabilitationsmaßnahme, vorliegt (vgl etwa BAG AP $\mathrm{Nr} 3$ zu $\$ 7$ LFZG). Die medizinische Rehabilitationsmaßnahme muss nicht stationär, sondern kann auch ambulant durchgeführt werden. Seit dem Inkrafttreten des SGB IX zum 1.7.2001 können auch ambulante Maßnahmen der medizinischen Rehabilitation einen Anspruch auf Entgeltfortzahlung begründen. Diese Änderung diente dazu, die Regelung des $\$ 9$ EFZG an $\$ 20$ SGB VI und $\$ 45$ SGB IX anzupassen, wonach während der Ausführung medizinischer Rehabilitationsmaßnahmen regelmäßig ein Anspruch auf Übergangsgeld dem Grunde nach gegeben ist und zwar unabhängig davon, ob die Leistung stationär oder ambulant erbracht wird.

b) Bewilligung durch einen öffentlich-rechtlichen Sozialleistungsträger: Wenn keine Bewilligung durch einen öffentlich-rechtlichen Sozialleistungsträger vorliegt, sondern die Kosten der Maßnahme von einem privaten Versicherungsunternehmen, einer karitativen Einrichtung oder sogar vom Arbeitnehmer selbst getragen werden, kann nunmehr auch ein Entgeltfortzahlungsanspruch bestehen. Voraussetzung ist aber, dass die Maßnahme von einem Arzt verordnet und in einer geeigneten Einrichtung durchgeführt wird.

c) Weitere Voraussetzung einer medizinischen Maßnahme iSd $\$ 9$ Abs 1 EFZG ist, dass die Maßnahme in einer Einrichtung der medizinischen Rehabilitation durchgeführt wird. Was darunter zu verstehen ist, ergib sich nicht unmittelbar aus dem EFZG. Anhaltspunkte können aus $\$ 107$ Abs 2 SGB V entnommen werden.

\section{Bernhard Albert}

\section{Schnittstellenmanagement bei medizinischer Rehabilitation in Österreich}

\section{Einleitung}

Medizinische Rehabilitation wird in Österreich nicht nur von allen Trägern der Zweige der SV durchgeführt, sondern auch von anderen Institutionen. Der Schwerpunkt liegt dabei aber doch im Bereich der SV. Aus diesem Grund wird dieser Bereich auch das Zentrum der folgenden Betrachtung darstellen.

Die Einleitung der Maßnahmen der medizinischen Rehabilitation ist in der Regel immer vom Eintritt des Versicherungsfalles oder vom Drohen des Eintritts eines Versicherungsfalles abhängig. Unterschiede existieren aber hinsichtlich der Ausgestaltung des Leistungsanspruches. Die Maßnahmen der medizinischen Rehabilitation können einerseits als Pflichtleistung mit einem individuell durchsetzbaren Rechtsanspruch, andererseits aber auch als Pflichtaufgabe ausgestaltet sein. Zweiteres zwingt den Träger lediglich zur Ausübung eines pflichtgemäßen Ermessens und eröffnet dem Leistungsempfänger keinen individuellen Rechtsanspruch. Nicht nur wegen des Umfanges der Leistung, sondern auch wegen der Vielfalt der Träger ist unter anderem die Frage der Zuständigkeit von essentieller Bedeutung.

\section{Zuständigkeit}

Die Zuständigkeit ist in den einzelnen Materiengesetzen geregelt. Das Bundesbehindertengesetz ${ }^{1}$ ) (BBG) enthält quasi als Grundsatzbestimmung diesbezüglich Vorschriften darüber, wie vorzugehen ist, wenn ein Antrag auf Rehabilitation beim unzuständigen Träger einlangt beziehungsweise wenn mehrere Träger zuständig sind.

Die Träger der gesetzlichen UV treffen Vorsorge für die Rehabilitation, wenn der Grund für die Behinderung ein Arbeitsunfall oder eine Berufskrankheit ist.

Die Träger der PV führen Maßnahmen der Rehabilitation dann durch, wenn die Behinderung ohne die Gewährung von Leistungen der Rehabilitation voraussichtlich zu Invalidität, Berufsunfähigkeit oder Erwerbsunfähigkeit führen würde oder bereits dazu geführt hat.

Die KV-Träger führen Maßnahmen der Rehabilitation in ergänzender $\mathrm{Zu}$ ständigkeit durch. Damit soll unter anderem auch für die stationäre medizinische

1) BGBl 1990/283 idF BGBl I 2008/109. 
Rehabilitation der in der PV nicht oder nicht mehr Anspruchsberechtigten gesorgt werden ${ }^{2}$ ).

\section{Leistungsumfang}

Die Maßnahmen der medizinischen Rehabilitation müssen in einem engen Konnex mit der primären medizinischen Behandlung der Versicherten gesehen werden. In der Regel findet zuerst eine Krankenbehandlung oder eine Unfallheilbehandlung statt, deren Ergebnis dann durch Maßnahmen der medizinischen Rehabilitation komplettiert werden soll. Schon unter diesem Aspekt betrachtet zeigt sich, dass ein enger Zusammenhang zwischen der primären medizinischen Behandlung und der Rehabilitation gegeben sein muss. Dieser Zusammenhang geht soweit, dass das Ergebnis der Rehabilitation durch Maßnahmen im Bereich der Erstversorgung positiv oder negativ beeinflusst werden kann.

Diesem Umstand wird dadurch Rechnung getragen, dass gem $\$ 5$ Abs 1 BBG gemeinsam mit dem potentiellen Rehabilitanden ein Gesamtplan über die Rehabilitation zu erstellen ist. An der Erstellung sollen nach Möglichkeit auch die behandelnden Institutionen der Erstversorgung mitwirken.

Betrachtet man im Zusammenhang mit der Frage des Leistungsumfanges die Systematik des $\mathrm{ASVG}^{3}$ ) so ist gem $\$ 133$ Abs 2 ASVG die Zielrichtung dieser Krankenbehandlung, dass die Gesundheit, die Arbeitsfähigkeit und die Fähigkeit, für die lebenswichtigen persönlichen Bedürfnisse zu sorgen, nach Möglichkeit wiederhergestellt, gefestigt oder gebessert werden soll. Die Krankenbehandlung umfasst die nötige ärztliche Hilfe, die Heilmittel und die Heilbehelfe. Sowohl für die Heilmittel als auch für die Heilbehelfe ist eine kostenmäßige Beteiligung des Versicherten vorgesehen. Die Maßnahmen der medizinischen Rehabilitation sollen gem $\$ 154$ a ASVG den Erfolg der Krankenbehandlung sichern oder die Folgen der Krankheit erleichtern. Durch die medizinische Rehabilitation soll der Gesundheitszustand der Versicherten oder der Angehörigen so weit wiederhergestellt werden, dass sie in der Lage sind, in der Gemeinschaft einen ihnen angemessenen Platz möglichst dauernd und ohne Betreuung und Hilfe einzunehmen. Zur Erreichung dieser Ziele kann ebenfalls ärztliche Hilfe, Heilmittel und Heilbehelfe gewährt werden.

Im Bereich der PV hat die Rehabilitation gem $\$ 300$ Abs 3 ASVG das Ziel, Behinderte bis zu einem solchen Grad ihrer Leistungsfähigkeit herzustellen oder wiederherzustellen, der sie in die Lage versetzt, im beruflichen und wirtschaftlichen Leben und in der Gemeinschaft einen ihnen angemessenen Platz möglichst dauernd einnehmen zu können.

Im Bereich der gesetzlichen UV werden die Maßnahmen der medizinischen Rehabilitation im Zuge der Unfallheilbehandlung gewährt. Die Unfallheilbehandlung hat mit allen geeigneten Mitteln die durch den Arbeitsunfall oder die Berufskrankheit hervorgerufene Gesundheitsstörung oder Körperbeschädigung sowie die durch den Arbeitsunfall oder die Berufskrankheit verursachte Min-

2) Rehakompass des ÖBIG, http://rehakompass.oebig.at/ (8. 10. 2008).

3) BGBI 1955/189 idF BGBl I 2008/146. derung der Erwerbsfähigkeit beziehungsweise der Fähigkeit zur Besorgung der lebenswichtigen persönlichen Angelegenheiten zu beseitigen oder zumindest zu bessern und eine Verschlimmerung der Folgen der Verletzung oder Erkrankung zu verhüten.

Im $\$ 154 a$ ASVG ist darüber hinaus festgelegt, dass die Maßnahmen der medizinischen Rehabilitation bei einem Träger der Pensions- oder der UV zu beantragen sind. Diese Träger sind verpflichtet, den Antrag unverzüglich an den Krankenversicherungsträger weiterzuleiten, sofern sie die Maßnahmen der medizinischen Rehabilitation nicht selber erbringen. In Abs 5 leg cit ist darüber hinaus eine Koordinationspflicht hinsichtlich der Maßnahmen der medizinischen Rehabilitation verankert. Diese Koordinationspflicht umfasst auch die Beachtung der entsprechenden RL des HV.

In dieser RL des HV sind einerseits die Voraussetzungen geregelt, unter denen Maßnahmen der Rehabilitation gewährt werden können oder müssen und andererseits enthält diese $\mathrm{RL}$ auch rudimentäre Bestimmungen über das Zusammenwirken der SV-Träger bei der Erledigung von Anträgen auf Rehabilitation.

\section{Ist-Status hinsichtlich Nahtstellenproblematik}

Grundsätzlich ergibt sich hinsichtlich der Nahtstellenproblematik daher folgendes Bild:

Úber den Bereich der SV hinaus erfolgt die Koordinierung der Maßnahmen der Rehabilitation grundsätzlich durch das BBG. Gem $\$ 2$ BBG haben die Träger der Rehabilitation ihre Maßnahmen insofern aufeinander abzustimmen, als eine unverzügliche Einleitung der Maßnahmen der Rehabilitation erforderlichenfalls sichergestellt werden muss. Wird ein Antrag auf Rehabilitation beim unzuständigen Träger eingebracht, so hat dieser Träger den Antrag unverzüglich an den zuständigen Träger weiterzuleiten. Darüber hinaus besteht sogar die Verpflichtung des unzuständigen Trägers, den zuständigen Träger von der Notwendigkeit von Rehabilitationsmaßnahmen zu informieren, wenn dieser Träger auf sonst eine Art und Weise von diesen Umständen Kenntnis erlangt. Es wird sogar soweit gegangen, dass bei Maßnahmen, die keinen Aufschub dulden, auch eine Leistungspflicht des unzuständigen Trägers gegen nachträglichen Kostenersatz entsteht.

$\operatorname{Im} \$ 5$ BBG ist festgelegt, dass der Rehabilitationsträger gemeinsam mit dem behinderten Menschen einen Gesamtplan hinsichtlich aller Maßnahmen der Rehabilitation zu erstellen hat. Besteht die Zuständigkeit von zwei oder mehreren Rehabilitationsträgern, so ist der Rehabilitationsplan im Zuge einer Teamberatung aufzustellen.

Für den Bereich der SV sind die Zuständigkeiten eindeutig in den Materiengesetzen geregelt. Zur darüber hinausgehenden Koordinierung hat der HV eine entsprechende RL-Kompetenz. Aufgrund dieser RL-Kompetenz wurde die RL für die Erbringung von Leistungen im Rahmen der Rehabilitation erlassen ${ }^{4}$ ). Durch diese RL erfolgt die Regelung der Voraussetzungen, nach denen in der Kranken- und PV Maßnahmen der medizinischen Rehabilitation erbracht werden können. Darüber hinaus ist auch das koordinierte Zusammenwirken der

\footnotetext{
$\left.{ }^{4}\right)$ www.sozdoc.at (8. 10. 2008).
} 
SV-Träger bei der Behandlung und Erledigung von Anträgen für diese Leistung Gegenstand dieser RL.

Durch diese Regelungen im BBG und in den entsprechenden Materiengesetzen ist eine Koordination der Rehabilitationsträger in formeller Hinsicht durchaus gegeben. Die Zuständigkeiten sind geklärt (wenn auch in einer komplexen Art und Weise) und eine effiziente Abwicklung von Anträgen ist gegeben.

Nicht wirklich geregelt ist aber der materielle Bereich. Der HV der SV-Träger nimmt insofern koordinierende Aufgaben wahr, als durch den Abschluss von Rahmenverträgen und die Erarbeitung von Strukturqualitätskriterien eine einheitliche Versorgung sichergestellt werden soll ${ }^{5}$ ). Diese Tätigkeiten beschränken sich aber tatsächlich weitgehend auf das Gebiet der Strukturqualität. Unter diesem Aspekt mutet auch der Hinweis im XXIV. Regierungsprogramm ${ }^{6}$ ) der neuen Bundesregierung etwas befremdlich an, der zum Thema Rehabilitation lediglich die Weiterentwicklung der stationären und ambulanten Rehabilitation und die Klärung der Zuständigkeiten auf Basis moderner Qualitätsstandards für alle $\mathrm{Al}-$ tersgruppen vorsieht. Die Frage der Zuständigkeit von Seiten der Rehabilitationsträger erscheint ausreichend geklärt. Nach meiner Ansicht weitgehend ungeklärt ist aber die Frage einer effizienten und ergebnisorientierten Durchführung der verschiedenen Maßnahmen der Rehabilitation und auch der medizinischen Rehabilitation. Hier schließt sich wieder der Kreis zur Erst- bzw Akutbehandlung: bereits zu diesem Zeitpunkt muss festgestellt werden, welche Bedürfnisse der Erkrankte oder Verletzte unter Berücksichtigung von zu erwartenden Dauerfolgen haben wird und wie diesem Defizit am besten beigekommen werden kann.

Gerade dieser Nahtstelle zwischen dem Erstversorgungsbereich und der anschließenden Rehabilitation muss eine vermehrte Aufmerksamkeit gewidmet werden. Die Frage der Qualitätskriterien im Bereich der medizinischen Rehabilitation lässt sich daher nicht nur auf die Fragen der Strukturqualität reduzieren sondern hat sich auch mit Fragen der Prozess- und Ergebnisqualität zu befassen. Nur durch die Berücksichtigung aller drei Kriterien können vorhandene Ressourcen optimal eingesetzt werden und es wird ökonomisch gesehen ein maximaler Output erreicht. Dies entspricht auch dem Gebot der OKonomie und Sparsamkeit.

Auf dem Schauplatz der medizinischen Rehabilitation sind daher folgende Akteure tätig: der Erkrankte oder Verletzte mit seinem Anspruch auf möglichste Wiederherstellung seines Gesundheitszustandes, die Institution der Erstversorgung, die SV-Träger als Rehabilitationsträger und auch als Financier der Abgeltung für verbleibende Folgen und jene Institution, in der Maßnahmen der medizinischen Rehabilitation durchgeführt werden können. Die Regelung der Berührungspunkte und Nahtstellen in diesem Bereich aus formeller Sicht ist gegeben, zumal für die potentiellen Akteure gemeinsame koordinierende Hierarchien vorhanden sind. Offen ist das Zusammenwirken mit den Bereichen Erstversorgung und Vertragspartner zur Erbringung von Rehabilitationsleistungen.

\footnotetext{
$\left.{ }^{5}\right)$ www.hauptverband.at/portal/index.html (21. 11. 2008).

6) www.bka.gv.at/DocView.axd?CobId=32965 (17. 2. 2009)
}

\section{Ideen für die Zukunft}

Für ein aus meiner Sicht effizientes Schnittstellenmanagement lassen Sie mich daher folgendes Szenario entwickeln:

Dem Verletzten selber ist es in der konkreten Situation unzumutbar die Tragweite des schicksalhaften Ereignisses und die Konsequenz daraus für die $\mathrm{Zu}$ kunft umfassend einschätzen zu können. Es muss daher jemand für den Verletzten die Koordinierung übernehmen.

Anbieten würde sich in diesem Zusammenhang ein externes Reha-Management, wie sich dieses in Deutschland bei Verkehrsunfällen bereits etabliert hat und in Einzelfällen auch von österreichischen Haftpflichtversicherern angeboten und verlangt wird. Dieses Reha-Management führt zu einer „Win-Win-Situation" für alle Beteiligten: die Chancen des Klienten auf eine möglichst rasche und größtmögliche Genesung sowie eine nachhaltige Wiedereingliederung in Arbeit, Beruf und soziales Umfeld werden erhöht; der eintrittspflichtigen Haftpflichtversicherung werden durch schnelle und bedarfsgerechte Versorgung des Klienten überflüssige, weil nicht immer zielgerichtete Aufwendungen erspart, ohne berechtigte Interessen des Klienten zu beschneiden ${ }^{7}$ ).

Bei diesem Reha-Management handelt es sich um eine soziale Dienstleistung bei der der Begriff der Qualität nicht nur eine ergebnisbezogene sondern auch immer eine prozessbezogene Dimension hat. Das heißt, es genügt nicht nur das Ziel einer Dienstleistung zu benennen, sondern auch der Prozess bis zur Erreichung dieses Zieles muss bestimmte Qualitätsanforderungen erfüllen ${ }^{8}$ ).

Auch für den Bereich der gesetzlichen SV würde sich die Einführung einer derartigen Institution mit gewissen Modifikationen anbieten. Als erster Schritt wäre es nötig, dass der Gesetzgeber eine Vereinheitlichung des Leistungsumfanges der medizinischen Rehabilitation in Angriff nimmt. Aus volkswirtschaftlicher Sicht ist es nicht zu begründen, warum für bestimmte Zweige der SV die Leistung der medizinischen Rehabilitation eine Pflichtleistung darstellt, für andere Bereiche lediglich eine Pflichtaufgabe. Auch die Unterscheidung des Leistungsumfanges im Hinblick auf die Unfallheilbehandlung und die Krankenbehandlung sind praktisch nicht nachvollziehbar.

Der volkswirtschaftliche Schaden ist vom zugrundeliegenden Versicherungsfall nicht abhängig. Durch die einheitliche Definition des Leistungsumfanges wird erreicht, dass auch einheitliche Qualitätskriterien nicht nur wie bisher auf dem Gebiet der Strukturqualität sondern auch auf dem Gebiet der Prozess- und Ergebnisqualität formuliert werden können. Wirtschaftlichkeit setzt Wirksamkeit und Qualität voraus. Vertragspartner oder eigene Reha-Einrichtungen der SV-Träger hätten sich dann in der Folge an diesen Kriterien zu orientieren und es wäre dadurch möglich, tatsächlich kontinuierlich qualitativ gleichbleibende Leistung zu bekannten Bedingungen bedarfsgerecht anzubieten. Der ökonomische Vorteil aus dieser Standardisierung ist vor allem in der bedarfsgerechten Leis-

$\left.{ }^{7}\right)$ Lauer, Qualitätsanforderung und Qualitätssicherung im Reha-Management, VersR 2008, $1465 \mathrm{ff}$

$\left.{ }^{8}\right)$ Heiner, Qualitätsentwicklung durch Evaluation (1996) 21. 
tungserbringung zu sehen: es können exakt jene Leistungen angeboten werden, die der konkrete Klient in seiner Situation benötigt.

Eine weitere nötige Komponente wäre aber auch die Begleitung des Prozesses durch Care- oder Casemanager. Ähnlich wie im Bereich der privaten Haftpflichtversicherungen könnten auch hier die vorhandenen und exakt definierten Ressourcen zielgerichtet und ökonomisch effizient eingesetzt werden.

Diesbezüglich wird im Bereich der SV kein Neuland beschritten, weil derartige "Rehabilitationsberater" oder "Sozialberater" durchaus bereits etabliert und mit großem Erfolg tätig sind. Nur als Beispiel sei auf die Initiative der SVA verwiesen, die gerade bei schweren Unfällen und Erkrankungen auf gute Erfolge ihres Modells zur Wiedereingliederung von Versicherten verweisen kann ${ }^{9}$ ), oder auch auf das Modell der AUVA, die ebenfalls die Koordinierung sämtlicher Zweige der Rehabilitation durch eigene Berater durchführt. Das Management der Schnittstellen zwischen den einzelnen Akteuren liegt daher in einer Hand.

Derartige Modelle bewähren sich nicht nur aufgrund der Ergebnisqualität sondern auch unter dem Gesichtspunkt der Ökonomie. Durch einheitliche Qualitätsstandards wird erreicht, dass Leistungen definierbar, beurteilbar und vergleichbar werden. Dies führt dazu, dass Leistungen ziel- und bedarfsgerecht appliziert werden können und dadurch auf den ersten Blick vielleicht höhere Aufwendungen im Bereich der Rehabilitation sich durch geringere Folgekosten egalisieren.

Gerade der Bereich der medizinischen Rehabilitation würde sich hier anbieten, zumal in diesem Bereich sehr oft die Rehabilitationsträger auch mit den Folgekosten direkt konfrontiert sind und daher eine betriebswirtschaftliche Gesamtbetrachtung der Fälle durchaus den ökonomischen Vorteil aufzeigen wird, zumal in diesem Bereich auch eine homogene Finanzierungsstruktur gegeben ist.

Nötig wäre ein derartiges Vorgehen gerade auch im Bereich der Erst- und Akutversorgung. Durch die komplexe Finanzierungssituation im Gesundheitswesen wird hier ein zielgerichteter Ressourceneinsatz aber ungleich schwerer zu realisieren sein.

\section{Zusammenfassung}

Zusammenfassend ist daher festzustellen, dass die Analyse der Ist-Situation hinsichtlich der Schnittstellenproblematik zeigt, dass die formelle Seite der medizinischen Rehabilitation vor allem im Hinblick auf Antragstellung und Antragbearbeitung und somit zu einem effizienten Zugang zu den Leistungen befriedigend geregelt ist.

Offen sind die Frage der Prozessqualität bei der Durchführung der Leistungen und auch die Frage der Überprüfung der Ergebnisqualität. Auch wenn durch das BBG festgelegt ist, dass in jedem Fall ein Rehabilitationsplan zu erstellen ist, bleibt das Problem der Klassifizierung der verschiedenen Leistungsanbieter gerade im Hinblick auf den konkreten Leistungsumfang. Faktisch wird daher auch im Bereich der medizinischen Rehabilitation eine Leistung erbracht werden, die am

9) Müller, SVAktuell 2008 (H 4) 4 ff. konkreten Bedürfnis vorbeigeht, wobei hier die Treffsicherheit sowohl nach unten als auch nach oben nicht gegeben sein wird. Dies ist durchaus auch dadurch zu begründen, dass gerade in diesem Bereich ein Nahtstellenmanagement nich wirklich gegeben ist.

Unbefriedigend ist nach wie vor auch der Unterschied im Leistungsumfang da für den Bereich der gesetzlichen UV durch den Umfang der Unfallheilbehandlung zumindest gesetzlich ein Unterschied zur Krankenbehandlung normiert ist. Faktisch spielt dieser Unterschied ohnedies kaum mehr eine Rolle, weil in den eigenen Einrichtungen der AUVA (sowohl Unfallkrankenhäuser als auch Rehabilitationszentren) allen Patienten faktisch die Unfallheilbehandlung gewährt wird, wobei gerade im Akutbereich die Auslastung mit Arbeitsunfällen gerade elf Prozent beträgt, Arbeitsunfälle aber auch in öffentlichen Krankenhäusern au dem Standard der Krankenbehandlung behandelt werden. Ein tatsächlicher Unterschied ist eigentlich nur mehr in der Gewährung von Hilfsmitteln und Heilbehelfen gegeben.

Als Ausblick für die Zukunft (und vielleicht ist das Regierungsprogramm ja so zu verstehen) wäre es jedenfalls nötig und auch zweckmäßig, den Leistungsumfang der medizinischen Rehabilitation für alle Zweige der SV einheitlich zu formulieren und gleichzeitig etwa im Wege einer RL-Kompetenz auch Qualitätskriterien nicht nur für die Strukturqualität sondern auch für die Prozess- und Ergebnisqualität zu formulieren. Für die Rehabilitationsträger als Financiers ergäbe sich dadurch die Möglichkeit, die Leistungsanbieter (egal ob eigene Einrichtungen oder Vertragspartner) hinsichtlich des Leistungsumfanges exakt einschätzen zu können und dadurch die entsprechende Leistung ziel- und bedarfsgerecht applizieren zu können.

Nicht zuletzt ist aufgrund der Komplexität der Fälle und des heterogenen Teilnehmerkreises am System der medizinischen Rehabilitation ein Case-Management nötig, um die definierten und angebotenen Leistungen bedarfsgenau applizieren zu können. Koordination und Fallmanagement sind aber als eine inter- und multidisziplinäre Aufgabe anzusehen. Auch wenn die Rehabilitation von spezialisierten Managern geleitet wird, sollte sie Gegenstand interdisziplinärer Leitlinien und Qualitätsstandrads sein ${ }^{10}$ )

Gerade ein derartiges Modell könnte aber auch als Beispiel für den Gesundheitsbereich insgesamt gesehen werden, wo derzeit noch Leistungen ohne Berücksichtigung des persönlichen Bedarfs erbracht werden und sowohl durch Über- als auch durch Unterversorgung unnötige Kosten verursacht werden. In diesem Sinne habe ich auch die Forderung von Welti verstanden, der eine rehabilitative Ausrichtung des gesamten Gesundheitswesens fordert ${ }^{11}$ ).

$\left.{ }^{10}\right)$ Welti, Perspektiven von Pflege und Sozialarbeit in der medizinischen Rehabilitation vor dem Hintergrund aktueller Entwicklungen im Gesundheitswesen, Fachtagung Pflege und Sozialarbeit, Schnittstellen in der med. Rehabilitation am 15. 7. 2007 (Kassel).

i1) Welti, aaO. 


\section{Eckart Stevens-Bartol}

\section{Schnittstellen im Recht der Rehabilitation in Deutschland}

\section{Einleitung}

Mein Beitrag zu diesem Thema befasst sich mit zwei Aspekten aus dem Mitte 2001 in Kraft getretenen neuen Recht der Rehabilitation in Deutschland, dem Band IX des Sozialgesetzbuchs der Bundesrepublik Deutschland (SGB IX). Diese sind:

- zum einen die Frage des Zusammenwirkens der Sozialleistungsträger untereinander bei der Erbringung einer Leistung zur Rehabilitation (vgl $\$ \$ 9$ bis 14 SGB IX, insb $\$ 14$ ), und

- zum anderen die Frage, wie das Problem der inzwischen unterschiedlichen Begriffe von Behinderung in den verschiedenen Sozialleistungsbereichen trotz gleichgebliebener Bewertungsregeln $(\$ 2$ SGB IX einerseits und $\$ 30$ Abs 17 BVG - Bundesversorgungsgesetz - andererseits) zu bewältigen ist.

\section{Die Probleme des Zusammenwirkens}

1. Das erste der beiden genannten Probleme ergibt sich aus dem Umstand, dass das System der Träger von Sozialleistungen, also auch der Träger von Leistungen zur Rehabilitation, in Deutschland stark gegliedert ist - das sog "gegliederte System der sozialen Sicherung“. Es kommen also sehr unterschiedliche und voneinander unabhängige Stellen zur Erbringung von Leistungen zur Rehabilitation in Betracht, ggf auch in Kooperation. Zugleich ist zu beachten, dass diese Rehabilitationsträger allesamt keine Stellen sind, die primär Aufgaben der Rehabilitation zu erfüllen haben, sondern in erster Linie jeweils Aufgaben eines der überkommenen Bereiche der sozialen Leistungen. Somit sind alle Rehabilitationsträger zugleich und in erster Linie Träger traditioneller sozialer Leistungen.

Dieser Zustand kommt in den $\$ \$ 5$ und 6 SGB IX deutlich zum Aus$\left.\operatorname{druck}^{1}\right)$.

1) \$ 5 SGB IX lautet:

„Leistungsgruppen. Zur Teilhabe werden erbracht

1. Leistungen zur medizinischen Rehabilitation,

2. Leistungen zur Teilhabe am Arbeitsleben,

3. unterhaltssichernde und andere ergänzende Leistungen,

4. Leistungen zur Teilhabe am Leben in der Gemeinschaft."

$\$ 6$ SGB IX lautet:

„(1) Träger der Leistungen zur Teilhabe (Rehabilitationsträger) können sein 
2. Dabei sollen die Leistungsträger bei der Erbringung der Leistungen zur Rehabilitation im Wesentlichen zwei Aspekte beachten, nämlich

- zum einen das Problem, wie die Kooperation der Behörden untereinande bei der Erbringung von Rehabilitationsleistungen organisiert werden soll,

- zum anderen die Klärung der Frage, wer im konkreten Fall eine Rehabilitationsleistung erbringen muss, wer also für eine Leistung zur Rehabilitation eigentlich zuständig ist.

Die hier zuerst genannte Frage regeln hauptsächlich die $\$ \$ 10-13$ des Gesetzes; diese Bestimmungen sollen hier außerhalb der Betrachtung bleiben. Die Frage der Zuständigkeit, mit der ich mich in erster Linie befassen möchte, regelt $\$ 14$.

3. Die Frage der Zuständigkeit des $\$ 14$ SGB IX ist in meinen Augen ein zentraler Punkt der „Schnittstellenproblematik“.

Selbstverständlich bestimmt jedes sozialrechtliche Leistungsgesetz schon dadurch, dass es den einzelnen Sozialleistungsträgern, die auch in $\$ 6$ SGB IX genannt sind, konkrete Aufgaben und die Pflicht zur Erbringung konkreter soziale Leistungen zuweist, zugleich auch deren sachliche Zuständigkeit.

Daran will auch das SGB IX nichts ändern ${ }^{2}$ ). Doch diese Zuständigkeitsregelung birgt für die Erbringung von Rehabilitationsleistungen - und für die Interessen des nach etwaigen Rehabilitationsleistungen nachfragenden Bürgers - Probleme. Denn der Umstand, dass $\$ 6$ SGB IX eine ganze Reihe von Rehabilitationsträgern nennt, führt bei diesen schnell zu der Versuchung, einen Antrag auf Rehabilitation abzulehnen mit der Begründung, für die geforderte Leistung sei eine andere Stelle zuständig. Einem solchen „negativen“ Kompetenzkonflikt soll nun $\$ 14$ SGB IX abhelfen ${ }^{3}$ ).

1. die gesetzlichen Krankenkassen für Leistungen nach $\$ 5 \mathrm{Nr}$. 1 und 3

2. die Bundesagentur für Arbeit für Leistungen nach $\$ 5 \mathrm{Nr}$. 2 und 3

2. die Bundesagentur für Arbeit für Leistungen nach $\$ 5 \mathrm{Nr}$. 2 und 3 ,
3. die Träger der gesetzlichen Unfallversicherung für Leistungen nach $\$ 5 \mathrm{Nr}$. 1 bis 4

4. die Träger der gesetzlichen Rentenversicherung für Leistungen nach $\$ 5 \mathrm{Nr}$. 1 bis 3 , die Träger der Alterssicherung der Landwirte Leistungen nach $\$ 5 \mathrm{Nr}$. 1 und 3 ,

5. die Träger der Kriegsopferversorgung und die Träger der Kriegsopferfürsorge im Rahmen des Rechts der sozialen Entschädigung bei Gesundheitsschäden für Leistungen nach $\$ 5 \mathrm{Nr}$. 1 bis 4 ,

6. die Träger der öffentlichen Jugendhilfe für Leistungen nach $\$ 5 \mathrm{Nr} .1,2$ und 4 ,

7. die Träger der Sozialhilfe für Leistungen nach $\$ 5 \mathrm{Nr}$. 1,2 und 4."

$\left.{ }^{2}\right)$ Denn $\$ 7$ SGB IX bestimmt dazu:

„Vorbehalt abweichender Regelungen. Die Vorschriften dieses Buches gelten für die Leistungen zur Teilhabe, soweit sich aus den für den jeweiligen Rehabilitationsträger geltenden Leistungsgesetzen nichts Abweichendes ergibt. Die Zuständigkeit und die Leistungen zur Teilhabe richten sich nach den für den jeweiligen Rehabilitationsträger geltenden Leistungsgesetzen."

$\left.{ }^{3}\right) \$ 14$ lautet ua:

,(1) Werden Leistungen zur Teilhabe beantragt, stellt der Rehabilitationsträger innerhalb von zwei Wochen nach Eingang des Antrages bei ihm fest, ob er nach dem für ihn geltenden Leistungsgesetz für die Leistung zuständig ist. ... Stellt er bei der Prüfung fest,
4. Diese Vorschrift verfolgt im Wesentlichen zwei Ziele:

- zum einen schnell Klarheit schaffen hinsichtlich der Zuständigkeit für die Leistungserbringung, der Antragsteller soll nicht beliebig "weitergereicht“ werden können, sondern nur ein $\mathrm{Mal}$;

- zum anderen zu erreichen, dass die Leistungserbringung schnell beginnen kann.

5. Nur wenn erreicht werden kann, dass die Antragsschrift einer Rehabilitationsakte nicht beliebig viele Eingangsstempel trägt, sondern allenfalls zwei, und wenn der Zwang besteht, dass das Verfahren schnell durchgeführt wird, sind die Ziele eines modernen Rehabilitationsrechts zu erreichen. Beides hängt eng zusammen; nur wenn schnell klar ist, wer zuständig ist, kann auch schnell geleistet werden.

Die Zahl der Eingangsstempel kann allerdings nur dann wirksam reduziert werden, wenn in Kauf genommen werden kann, dass nicht in jedem Falle die in der Sache tatsächlich zuständige Behörde gesucht wird; es muss möglich sein dass im Einzelfall auch mal eine der Sache nach unzuständige Stelle tätig wird. Da das Gesetz also will, dass mit der Stellung eines Antrages auf eine Leistung zur Rehabilitation ein sehr kurzfristiges Verfahren in Gang gesetzt wird, an dessen baldigem Abschluss in der Regel die Erbringung einer Leistung zur Rehabilitation steht, nimmt das Gesetz im Ergebnis die Fiktion der Zuständigkeit, gegebenenfalls auch entgegen einer „an sich“, der Sache, nach gegebenen Zuständigkei in Kauf.

6. Daraus ergeben sich wiederum drei Schwierigkeiten, die man mit folgenden Fragen beschreiben kann:

1. Wenn so eine unzuständige Stelle eine Leistung zur Rehabilitation erbringen muss, ist sie dann immer noch unzuständig, oder ist sie dann - auch - zuständig?

2. Nach welchen Rechtsvorschriften richtet sich der Inhalt einer solchen Leistung zur Rehabilitation?

3. Wer zahlt schließlich?

$\mathrm{Zu}$ 1.: Das BSG ${ }^{4}$ ) hat dazu klargestellt, dass ein wegen $\$ 14$ SGB IX zuständig gewordener Leistungsträger, der der Sache nach „eigentlich“ unzuständig war, gleichwohl aber geleistet hat, nun nicht mehr "unzuständiger" Leistungsträger ist, sondern „nachrangiger" Leistungsträger.

$\mathrm{Zu} 2 .:$ Diese Frage beantwortet $\$ 7$ SGB IX ${ }^{5}$ ). Dies bedeutet ua, dass ein gem $\$ 14$,nachrangig“ zuständig gewordener Leistungsträger nur Leistungen erbringen kann von der Art, die zu seinem ursprünglichen Zuständigkeitsbereich gehören. Dazu steht es allerdings nicht in Widerspruch, dass bei der Durchführung

dass er für die Leistung nicht zuständig ist, leitet er den Antrag unverzüglich dem nach seiner Auffassung zuständigen Rehabilitationsträger zu. ...

(2) Wird der Antrag nicht weitergeleitet, stellt der Rehabilitationsträger den Rehabilitationsbedarf unverzüglich fest. ... Wird der Antrag weitergeleitet, geiten die Sätze n 1 und 2 für den Rehabilitationsträger, an den der Antrag weitergeleitet worden ist, entsprechend."

) Vgl BSG 26. 6. 2007 - B 1 KR 34/06 R.

$\left.{ }^{5}\right)$ Vgl oben FN 2. 
dieser Leistungen nicht nur die Ziele der traditionellen Bücher des Sozialgesetzbuchs verfolgt werden sollen, sondern auch die spezifischen Ziele des Rechts der Rehabilitation des SGB IX $\left.{ }^{6}\right),{ }^{7}$ ).

$\mathrm{Zu}$ 3.: Zahlen muss am Ende nicht der nachrangig zuständige Leistungsträger, sondern der nach dem jeweiligen Sozialleistungsgesetz zuständige Leistungsträger, der „primär“ zuständige. Die bestimmt $\$ 14$ Abs 4 SGB IX.8)

7. Im Ergebnis gilt: Wenn jemand beispielsweise bei seiner GKV, zB der Allgemeinen Ortskrankenkasse (AOK) etwa eine Kur beantragt, so muss die KV innerhalb von zwei Wochen darüber entscheiden,

- ob sie selbst zuständig ist oder

- ob ein anderer Träger zuständig ist und

- wenn ja, welcher.

Hält sich die Krankenkasse als zuerst angegangene Stelle selbst für zuständig, muss sie zugleich auch über den Rehabilitationsbedarf in der Sache entscheiden, also prüfen, ob die Leistung zu Recht beantragt worden ist.

8. Kurz gefasst bedeutet dies: Leitet der zuerst angegangene Träger, also im Beispiel die Krankenkasse, den Fall vor Ablauf der zwei Wochen an einen anderen Träger $-\mathrm{zB}$ die Rentenversicherung - weiter, hat diese über den Rehabilitationsbedarf zu entscheiden; weiterleiten darf sie den Antrag nun nicht mehr. Außerdem muss der andere Träger, im Beispiel also die Rentenversicherung, ebenfalls „unverzüglich“ darüber entscheiden, ob die beantragte Leistung nach dem für sie geltenden Recht zu gewähren ist. Erbringt sie die Leistung und war sie primär zuständig, ist alles in Ordnung. War sie nur "nachrangig“ zuständig aufgrund von $\$ 14$ SGB IX, kann sie vom primär zuständigen Träger Erstattung fordern.

9. Der erstangegangene Träger hat also folgende Möglichkeiten:

- er kann leisten, wenn er sich für zuständig und den Antrag auf die Reha-

Leistung für begründet hält

- er kann die Zwei-Wochen-Frist versäumen (dann ist er - nachrangig - zuständig)

- er kann, wenn er einen anderen für zuständig hält, fristgemäß weiterleiten.

- er kann die beantragte Leistung ablehnen, wenn er den geltend gemachten Anspruch für gänzlich nicht gerechtfertigt hält, auch nicht gegenüber einem anderen Träger.

Leitet der Erstangegangene weiter und gibt es folglich einen Zweitangegangenen, so kann dieser nur

- leisten

- oder ablehnen.

$\left.{ }^{6}\right) S \S 7$ Satz 1 (oben FN 2) und Feldes ua SGB IX (2009) $\$ 7$ Rn 7.

$\left.{ }^{7}\right)$ Nunmehr fordert das BSG - 21.8. 2008 - B 13 R 33/07 R - auch, dass der nachrangig zuständige Träger alle in Betracht kommenden Anspruchsgrundlagen zu berücksichtigen hat, auch solche, für die er „eigentlich“ gar nicht zuständig ist.

$\left.{ }^{8}\right)$ Dieser lautet:

„Wird nach Bewilligung der Leistung durch einen Rehabilitationsträger nach Abs. 1 Satz 2 bis 4 festgestellt, dass ein anderer Rehabilitationsträger für die Leistung zuständig ist, erstattet dieser dem Rehabilitationsträger, der die Leistung erbracht hat, dessen Aufwendungen nach den für diesen geltenden Rechtsvorschriften."
Etwas anderes gibt es grundsätzlich nicht. Die Rechtslage ist also interessant, wenig übersichtlich, und jede Entscheidung steht unter beträchtlichem Zeitdruck.

10. Zur Veranschaulichung ein Beispiel:')

Eine Versicherte - zuletzt tätig als selbständige Ausfahrerin von Legehennen, davor beschäftigt als Arbeiterin in abhängiger Stellung, und zwischendurch Hausfrau - ist Mitglied in der gesetzlichen Rentenversicherung sowie der AOK (allgemeine Krankenkasse) aus ihrer früheren abhängigen Erwerbstätigkeit. In der Krankenkasse ist sie, da sie jetzt selbständig arbeitet, inzwischen freiwillig versichert. In die gesetzlich Rentenversicherung hat sie etwas mehr als fünf Jahre reguläre Beiträge bezahlt, sodass hier die Versicherungszeiten für eine Altersrente im Zeitpunkt des Erreichens des regulären Rentenalters (derzeit 65, künftig ansteigend bis 67) ausreichen würden ${ }^{10}$ ).

Sie beantragt nunmehr bei der Krankenkasse die Gewährung einer stationären Maßnahme zur medizinischen Rehabilitation, also einer Kur.

Die Krankenkasse prüft schnell und auch fristgemäß iSd \$14 SGB IX die Erforderlichkeit und Geeignetheit der beantragten Maßnahme. Der wegen der zugrundeliegenden medizinischen Fragen sodann auch noch befasste Vertreter des medizinischen Dienstes der Krankenkasse (hierfür räumt das Gesetz in $\$ 14$ SGB IX noch extra eine Frist von weiteren drei Wochen ein) bescheinigt dann allerdings, dass es eine stationäre Maßnahme nicht bräuchte, sondern dass eine ambulante Maßnahme möglich und ausreichend sei, und dass daher ein Anspruch auf eine stationäre Maßnahme nach den für die Krankenkasse maßgeblichen gesetzlichen Bestimmungen des SGB $V^{11}$ ) nicht bejaht werden könne. Eine Kur wie beantragt komme also nicht in Frage, es fehle an der Erforderlichkeit. Es würde ausreichen - und wäre auch viel billiger -, wenn die Antragstellerin regelmäßig von zuhause aus die erforderlichen Anwendungen aufsuchen würde.

Daraufhin - und wohl auch vor dem Hintergrund des drohenden Ablauf der knappen Fristen des $\$ 14$ SGB IX von nunmehr insgesamt fünf Wochen, zwei Wochen plus drei Wochen für die medizinische Begutachtung - sieht der Sachbearbeiter der KV davon ab, das Problem zur weiteren Klärung noch mit der Antragstellerin zu erörtern, was er nach dem Gesetz eigentlich müsste ${ }^{12}$ ).

Offenbar weil der Sachbearbeiter der Krankenkasse aber hier das Gefühl hat, dass die Antragstellerin auch ein bisschen Recht hat, sieht er sich gehindert, den

9) Aus der gerichtlichen Mediation am Bayer LSG; ein uU zeitsparender, kostenloser Service der Bayerischen Sozialgerichtsbarkeit für Parteien, die weiter denken.

$\left.{ }^{10}\right) \$ 35$ SGB VI:

„Regelaltersrente. Versicherte haben Anspruch auf Regelaltersrente, wenn sie 1. die Regelaltersgrenze erreicht und 2. die allgemeine Wartezeit erfültt haben ..."

$\$ 50$ SGB VI:

"(1) Die Erfüllung der allgemeinen Wartezeit von fünf Jahren ist Voraussetzung für einen Anspruch auf 1. Regelaltersrente 2...."

${ }^{11)}$ Das Recht der GKV.

12) Vgl $\$ 9$ SGB IX:

„Bei der Entscheidung über die Leistungen und bei der Ausführung der Leistungen zur Teilhabe wird berechtigten Wünschen der Leistungsberechtigten entsprochen ..." 
Antrag jetzt einfach abzulehnen; er kann sich - vor dem Hintergrund der Stellungnahme seines medizinischen Dienstes - aber auch nicht dazu durchringen, die stationäre Maßnahme zu bewilligen. Er wählt nun also gerade noch fristgemäß den ihm als Rettung erscheinenden Ausweg, nämlich er leitet den Fall an die Rentenversicherung weiter.

Die Rentenversicherung kann jetzt den Fall weder zurückschicken, noch weiterleiten; sie ist „dran“. Sie prüft also nun, ob bei der Antragstellerin die versicherungsrechtlichen Voraussetzungen nach dem für die Rentenversicherung maßgebenden Recht $^{13}$ ) für eine Maßnahme der medizinischen Rehabilitation - also eine Kur - gegeben sind. Dabei kommt sie zunächst zu dem Ergebnis, dass die nach dem Recht der Rentenversicherung für eine „Leistung zur Teilhabe“, also auch für unsere Kur, geforderte Wartezeit von 15 Jahren nicht erfüllt is $\mathrm{t}^{14}$ ); auch die alternativ dazu aufgestellte Voraussetzung, dass die Antragstellerin schon Rente wegen verminderter Erwerbsfähigkeit bezieht, liegt nicht vor; die Antragstellerin bezieht keine Rente wegen verminderter Erwerbsfähigkeit und hat - vor ihrer jahrelangen Tätigkeit als Hausfrau - nur die allgemeine Wartezeit der Rentenversicherung (von 5 Jahren) erfüllt, und die reicht hier nicht aus ${ }^{15}$ ).

Allerdings - so ergibt die Prüfung bei der Rentenversicherung weiter - käme vielleicht die Erfüllung der versicherungsrechtlichen Voraussetzungen für einen Sonderfall - nämlich nach $\$ 11$ Abs 2 S 1 SGB VI - in Betracht, aber nur dann, wenn bei der Antragstellerin schon „verminderte Erwerbsfähigkeit“ bestünde oder wenigstens drohte. Wäre dies der Fall, so würde hier die Erfüllung der allgemeinen Wartezeit von 5 Jahren genügen ${ }^{16}$ ).

Anders als bei der Prüfung der Krankenkasse, wo es um Heilung oder Stabilisierung geht, kommt es hier also auf das Maß der Erwerbsfähigkeit bzw von deren Beeinträchtigung an - und natürlich, wie bei allem in der Rentenversicherung -, auf die vorgeschriebene Wartezeit.

Aber nach dem Ergebnis des Gutachtens des inzwischen ebenfalls zu Rate gezogenen medizinischen Dienstes des Rentenversicherungsträgers sei eine Be-

Nach $\$ 1$ SGB IX sollen Rehabilitationsleistungen bei den Betroffenen die Selbstbestimmung und gleichberechtigte Teilhabe fördern. Dazu passt es überhaupt nicht, wenn solche Leistungen über die Köpfe der Betroffenen hinweg schlicht angeordnet werden.

$\left.{ }^{13}\right)$ SGB VI.

$\left.{ }^{14}\right) \$ 11$ Abs 1 SGB VI:

(1) Für Leistungen zur Teilhabe haben Versicherte die versicherungsrechtlichen Voraussetzungen erfüllt, die bei Antragstellung

1. die Wartezeit von 15 Jahren erfüllt haben oder

2. eine Rente wegen verminderter Erwerbsfähigkeit beziehen. ...“

${ }^{15}$ ) Eigentlich war der Ausgang spätestens hier klar, sofern man davon absieht, dass schon die Krankenkasse klare Verhältnisse vorgefunden hatte.

$\left.{ }^{16}\right) \$ 11$ Abs 2 S 1 SGB VI

Für die Leistungen zur medizinischen Rehabilitation haben Versicherte die versicherungsrechtlichen Voraussetzungen auch erfüllt, die

$1 . .$.

2....

3. vermindert erwerbsfähig sind oder bei denen dies in absehbarer Zeit zu erwarten ist, wenn sie die allgemeine Wartezeit erfüllt haben." einträchtigung der Erwerbsfähigkeit hier nicht der Fall, die Antragstellerin sei ohne weiteres in der Lage, täglich unter den Bedingungen des allgemeinen $\mathrm{Ar}$ beitsmarktes wenigstens sechs Stunden - irgendwas - zu arbeiten ( $\$ 43$ Abs $1 \mathrm{~S} 2$ SGB VI) ${ }^{17}$ ). Das ist nämlich der Maßstab des Rechts der Rentenversicherung für die Frage, was „Erwerbsminderung" ist. Auf den Beruf kommt es dabei nicht an auf die Höhe des erzielten Geldes auch nicht, und als Erwerb zählt alles, was der sog "allgemeine Arbeitsmarkt" hergibt.

Dass die Antragstellerin inzwischen als selbständige Ausfahrerin von Legehennen tätig ist, ändert daran nichts. Denn - so der Sachbearbeiter der Rentenversicherung zu Recht - es komme an dieser Stelle wegen $\$ 43$ SGB VI nur auf den allgemeinen Arbeitsmarkt an; Berufsschutz komme nicht in Betracht; die versicherungsrechtlichen Voraussetzungen müssten daher hier verneint werden.

Die nun - oder eigentlich von Anfang an - auf der Hand liegende Konsequenz, den Antrag abzulehnen, zieht aber auch der Rentenversicherungsträge nicht. Dabei mag die Überlegung entscheidend gewesen sein, dass er ja nun gemäß $\$ 14$ SGB IX irgendwie „zuständig" geworden sei. Und auch er hat wohl den Eindruck gewonnen, die Versicherte habe auch ein bisschen Recht. Dass seine Zuständigkeit nach $\$ 14$ des SGB IX als nachrangige hier nur eine "formale" ist, die ihn als solche in der Sache zu gar nichts verpflichtet, gerät dabei zu Unrech aus dem Blickfeld.

Der Sachbearbeiter der Rentenversicherung prüft jetzt also unter Absehen von den für ihn geltenden versicherungsrechtlichen Voraussetzungen weiter, und stellt fest, dass zwar nicht die versicherungsrechtlichen Voraussetzungen des Rentenversicherungsrechts erfüllt sind, dass aber doch die persönlichen Voraussetzungen der Versicherten speziell für eine Leistung zur medizinischen Rehabilitation bejaht werden könnten, nämlich gemäß $\$ 10 \mathrm{Abs} 1 \mathrm{Nr} 2$ lit a SGB VI. Denn dort ist Tatbestandsmerkmal für eine Maßnahme zur medizinischen Rehabilitation nicht „Erwerbsminderung", was nach zutreffender Ansicht des Rentenversicherungsträgers immer zu der Begrifflichkeit des $\$ 43$ SGB VI führt und daher verneint werden muss, sondern bloß "Gefährdung der Erwerbsfähigkeit", wobei die konkrete Erwerbstätigkeit der Versicherten als Maßstab diene, also hier die Tätigkeit als Ausfahrerin von Legehennen ${ }^{18}$ ).

$\left.{ }^{17}\right) \$ 43$ Abs 1 S 2 SGB VI

„... Teilweise erwerbsgemindert sind Versicherte, die wegen Krankheit oder Behinderung auf nicht absehbare Zeit außerstande sind, unter den üblichen Bedingungen des allgemeinen Arbeitsmarktes mindestens 6 Stunden täglich erwerbstätig zu sein.“

${ }^{18)} \$ 10$ Abs $1 \mathrm{Nr} 2$ lit a SGB VI.

„Persönliche Voraussetzungen.

(1) Für Leistungen zur Teilhabe haben Versicherte die persönlichen Voraussetzun gen erfüllt,

1

2. bei denen voraussichtlich

a) bei erheblicher Gefährdung der Erwerbsfähigkeit eine Minderung der Erwerbsfähigkeit durch Leistungen zur medizinischen Rehabilitation oder zur Teilhabe am Arbeitsleben abgewendet werden kann

b) .... 
Diese sei in der Tat gefährdet.

Die versicherungsrechtlichen Voraussetzungen für eine Leistung des Trägers der Rentenversicherung fehlen aber nach wie vor, denn bei genauerer Betrachtung käme man hier an der Bedingung der 15-jährigen Vorversicherungszeit wieder nicht vorbei, siehe oben.

Unter Absehen vom Fehlen der versicherungsrechtlichen Voraussetzungen, aber auch mit dem Gefühl, dass eigentlich ja auch die Krankenkasse etwas hätte bezahlen müssen, bewilligt nun der Rentenversicherungsträger die beantragte Kur, und verlangt sodann Erstattung der Kosten von der Krankenkasse. Im Verfahren vor dem Sozialgericht (Rentenversicherung gegen Krankenkasse) vereinbart man schließlich, das Kostentragungsproblem durch Mediation lösen zu wollen. Die Kur hat inzwischen natürlich - zum Glück, möchte man sagen - längst stattgefunden.

Das Ergebnis der Mediationsverhandlung liegt auf der Hand: Der Umstand, dass die Kur überhaupt nicht hätte bewilligt werden dürfen, gerät dabei in den Hintergrund, weil er jetzt zu nichts mehr führt. Dass die Krankenkasse unter diesen Umständen gar nichts hätte bezahlen müssen, wird verdrängt, denn irgendwie will man jetzt ja nur noch „die Kuh vom Eis“ holen. Also erklärt sich die Krankenkasse bereit, die Kosten der ambulanten Maßnahme zu zahlen, die Rentenkasse zahlt den Rest. Zufällig macht das bei großzügiger Betrachtung jeweils ungefähr die Hälfte der ganzen Kosten aus, man einigt sich also in der Mitte.

Als Nebenprodukt der Mediationsverhandlung kommt noch ans Licht, dass keiner der beiden Sachbearbeiter auf die Idee gekommen war, den anderen einfach mal anzurufen oder ihm gar die Akten oder wenigstens Teile davon zur Verfügung zu stellen. Dass das geschehen soll, steht aber ausdrücklich im Gesetz ( $\$ 12$ Abs 1 Nr 2 SGB IX $\left.{ }^{19}\right)$ ). Außerdem gibt es „gemeinsame Empfehlungen “ gem $\$ 13$ SGB IX, in denen dies genauestens geregelt ist. Dass es diese "gemeinsamen Empfehlungen" gibt, wussten beide Sachbearbeiter; da aber auch mal hineinzuschauen, dazu waren sie noch nicht gekommen.

11. Aber vergessen wir nicht: Die Krankenkasse hätte nicht leisten dürfen, wenn sie sich nicht über das Urteil ihres Arztes hinwegsetzen wollte. Sie hätte aber auch nicht weiterleiten dürfen, denn für die von der Antragstellerin geforderte Leistung gab es auch in anderen Rechtsbereichen keine tragfähigen Voraussetzungen, insbesondere nicht im Recht der gesetzlichen Rentenversicherung (siehe oben). Die Rentenversicherung hätte dementsprechend ebenfalls nich leisten dürfen. Auch sie hätte ablehnen müssen. Die Antragstellerin hatte halt einfach keinen Anspruch auf eine Kur. Für einen Erstattungsanspruch war dann eigentlich auch kein Raum.

Nur: Die persönlichen Vorsaussetzungen sind hier kumulativ zusammen mit den versicherungsrechtlichen Voraussetzungen vonnöten!

${ }^{19}$ ) „Zusammenarbeit der Rehabilitationsträger

(1) Im Rahmend er durch Gesetz, Rechtsverordnung oder allgemeine Verwaltungsvorschrift getroffenen Regelungen sind die Rehabilitationsträger verantwortlich, dass

1. ....

2. Abgrenzungsfragen einvernehmlich geklärt werden

3.
12. ME genügt es hier nicht, für das Ergebnis den durch die kurzen Fristen erzeugten Zeitdruck verantwortlich zu machen bzw das sog „gegliederte System der sozialen Sicherung".

Für die Erklärung der scheinbaren Verwirrung bei den Akteuren muss wohl noch ein weiterer Aspekt ins Blickfeld kommen, der hier - neben allem anderen - offenbar eine zentrale Rolle spielt:

Es gibt in Deutschland kein einheitliches materielles Recht der Rehabilitationsleistungen, sondern es gilt der Grundsatz, dass jeder Leistungsträger nur diejenigen Leistungen erbringen kann, die nach dem für ihn geltenden Recht zum Katalog der von ihm zu erbringenden Leistungen gehören. Dies erscheint auch folgerichtig, wenn man bedenkt, dass die Gelder der einzelnen Träger der Sozialleistungen nicht aus einem Topf, sondern von unterschiedlichen Kreisen von versicherten Personen stammen, unterschiedlichen sozialen und politischen Zwecken dienen und unterschiedlich berechnet werden.

13. Deshalb ist auch festgelegt ( $\$ 7$ SGB IX, s oben FN 2), dass die im SGB IX versuchte weitgehende Angleichung der Rechtsprinzipien des Schwerbehindertenrechts ${ }^{20}$ ) im Bereich der Rehabilitation immer gelten, aber nicht auch die Rechtsgrundlagen der Leistungen zur Rehabilitation erfassen soll.

Jeder Leistungsträger darf daher im Endeffekt nur diejenigen Leistungen erbringen, die in seinem gesetzlichen Leistungskatalog vorgesehen sind, dh, die Krankenkassen dürfen nur Leistungen erbringen, die im SGB V vorgesehen sind, für die Arbeitsverwaltung gilt das SGB III, für die Träger der gesetzlichen Rentenversicherung ist alles geregelt im SGB VI usw.

Was jedoch das Verfahren - die Zielsetzung der Leistungen, den Umgang mit dem Antragsteller, die Festlegung und die Erbringung der Leistung zur Rehabilitation - angeht, so bestimmt das SGB IX für alle Träger das Gleiche. Es gilt dabei der Grundsatz: jeder kann zuständig sein, und sei es auch nur durch Fristversäumung oder Weiterleitung, aber niemand darf letztlich Leistungen erbringen, die nicht zu seinem gesetzlichen Leistungsangebot gehören.

14. $\mathrm{Zu}$ den Aufgaben, die der Sachbearbeiter des zuerst angegangenen Leistungsträgers zu bewältigen hat, gehört daher nicht nur die Beantwortung der Frage, ob er selbst zuständig ist, und wenn ja, wie die zu erbringende Leistung aussehen soll, sondern auch, wer - aus dem breiten Spektrum der Möglichkeiten (s \$\$ 5 und 6 SGB IX, vgl oben FN 1) - andernfalls zuständig wäre, und wofür.

Eigentlich müsste jeder Sachbearbeiter in diesem Bereich auch alle RehaLeistungen aller anderen Reha-Träger kennen und beurteilen können. Schon das Mediationsbeispiel und die nicht ganz einfachen Anspruchsvoraussetzungen im SGB VI dürften aber gezeigt haben, dass hier von den einzelnen Sachbearbeitern ziemlich viel gefordert ist. Der Sachbearbeiter des zuerst angegangenen Leistungsträgers steht also unter großem Zeitdruck vor der Frage, was er tun soll, wenn er das Gefühl hat, dass dem Antragsteller schon irgendwie geholfen werden

$\left.{ }^{20}\right)$ Va die Einbeziehung des Leistungsempfängers in den Entscheidungsprozess, die Koordination der einzelnen Leistungen der verschiedenen Träger untereinander, die Sicherstellung der Kongruenz der einzelnen Leistungen, die Pflicht zur Zusammenarbeit der verschiedenen Leistungsträger, $\$ \$ 9-13$ SGB IX. 
muss, wenn er aber in der kurzen zur Verfügung stehenden Zeit nicht recht entscheiden kann, wie und von wem.

15. Den im Beispiel auf den ersten Blick scheinbar simplen Ausweg, der hier dann ja auch gewählt worden ist, nämlich eine unerwünschte Sache einfach vor Ablauf der 14-Tages-Frist an einen anderen Träger weiter zu schieben, hat der Gesetzgeber eigentlich durch eine einfache Regelung unschädlich zu machen versucht. Er hat zunächst angeordnet, dass der andere Träger die Sache nun seinerseits weder zurückschicken noch seinerseits erneut weiterleiten darf. Dies erscheint sinnvoll. Denn jetzt hat ja immerhin eine mehr oder weniger kompetente Stelle die Zuständigkeit schon mal geprüft.

Sodann hat der Gesetzgeber in diesem Zusammenhang auch festgelegt, dass der Träger, der eine Leistung erbringt, formal dafür zwar zuständig ist, aber gleichwohl von dem, der die Leistung eigentlich hätte erbringen müssen, Kostenerstattung fordern kann.

16. Aus der Sicht eines Antragstellers befriedigt dies aber nur bedingt. Denn: Schiebt der zuerst angegangene Leistungsträger weiter, um dem Risiko, leisten zu müssen, zu entgehen, dann würde er sich zwar im Endeffekt die Kosten für die Rehabilitationsmaßnahme nicht unbedingt sparen können; dem Antragsteller würde dies aber dennoch in vielen Fällen einen großen Nachteil zufügen. Denn wenn dann der als zweiter angegangene Leistungsträger den Antrag - anders als in dem beschriebenen Mediationsfall - ablehnen würde, weil der zuerst angegangene hätte leisten müssen, müsste der Antragsteller den Zweiten verklagen, und dann versuchen, gegen den zuerst angegangenen Leistungsträger, der zu diesem Verfahren beigeladen werden muss, eine Verurteilung zu erwirken - für den Bürger kein sehr reizvoller Weg.

17. Was lässt sich aus all dem folgern?

1. die Förderung der Rehabilitation ist zu begrüßen;

2. die das Verfahren belebenden Fristen sind ebenfalls zu begrüßen;

3. die Beachtung der "gemeinsamen Empfehlungen“ des $\$ 13$ SGB IX muss gefördert werden;

4. Sozialleistungsbehörden sollten mehr mit den Betroffenen reden;

5. Sozialleistungsbehörden sollten mehr untereinander reden;

6. Sozialleistungsbehörden sollten genug Personal haben, um in der Lage zu sein, auch schnell und gründlich genug zu arbeiten

7. der Möglichkeit, eine Sache weiter zu schieben, auch auf die Gefahr hin, nach längerer Zeit von einem anderen Träger auf Erstattung in Anspruch genommen zu werden, müsste die Attraktivität genommen werden.

\section{Der Begriff der Behinderung}

1. Hier geht es um die Frage, ob die Regeln, die für die Bewertung des Grade einer Behinderung gelten, und die im Grunde noch immer auf der Gedankenwelt des alten Schwerbeschädigtenrechtes beruhen, gleichwohl - wie es $₫ 69$ SGB IX $^{21}$ )

$\left.{ }^{21}\right) \$ 69$ Abs 1 SGB IX lautet ua:

„(1) Auf Antrag des behinderten Menschen stellen die für die Durchführung des Bundesversorgungsgesetzes zuständigen Behörden das Vorliegen einer Behinderung und anordnet - auch im neuen Recht der Rehabilitation des SGB IX verwendet werden können.

2. Hier stellen sich $m E$ folgende Fragen:

1. Geht es wirklich an, für die Maßstäbe, nach denen im Recht des SGB IX bewertet werden soll, wie hoch das Ausmaß einer Behinderung einzuschätzen ist, auf das Recht des Bundesversorgungsgesetzes (BVG) - also das Gesetz, das die Ansprüche von Kriegsopfern regelt - zurückzugreifen?

2. Muss man, nachdem das deutsche Behindertenrecht des SGB IX sich nun internationalen Maßstäben anzugleichen begonnen hat, tatsächlich noch nach Zehnergraden bewerten - und damit den Begriff des „schwerbehinderten Menschen“ - wenigstens $50 \%$ - fortschreiben, der in unserem Recht eine große Rolle spielt, oder genügt es nicht - wie es im sozialrechtlichen Teil des SGB IX inzwischen ohnehin der Fall ist - entsprechend internationalem Recht nur noch mit dem Begriff der Behinderung zu operieren?

Während ich mich mit der zweiten Frage hier nicht weiter beschäftigen kann, soll die erste Frage ausführlicher behandelt werden.

3. An dieser Stelle erscheint mir ein Blick auf die historische Entwicklung des Begriffs der Behinderung bzw Schwerbehinderung im deutschen Recht sinnvoll.

Ausgangspunkt des Behindertenrechts war in Deutschland die Notwendigkeit, Kriegsfolgen zu bewältigen. So war es eine der ersten Aufgaben der Weimarer Republik auf sozialpolitischem Gebiet, die im Ersten Weltkrieg beschädigten Menschen zu versorgen und zu integrieren. Dies geschah bereits im Januar 1919 durch eine "Schwerbeschädigtenverordnung“, die dann im Jahre 1920/1923 durch ein Gesetz zur Beschäftigung Schwerbeschädigter abgelöst wurde. Ziel war die schwerbeschädigten Menschen wieder in das Arbeitsleben zu integrieren. Hierzu sollten ein behördlich kontrollierter Einstellungszwang und ein behördlich abgesicherter Kündigungsschutz dienen. Als Schwerbeschädigte galten nich sämtliche Menschen mit gesundheitlichen Einschränkungen, sondern nur solche, deren Beschädigung bestimmte Ursachen hatte, nämlich Kriegsfolgen und Arbeitsunfälle ${ }^{22}$ ). Ein irgend geartetes Präventionsziel, wie es heute das SGB IX

den Grad der Behinderung fest. Beantragt eine erwerbstätige Person die Feststellung der Eigenschaft als schwerbehinderter Mensch ( $\$ 2$ Abs. 2), gelten die in $\$ 14$ Abs. 2 Satz 2 und 4 sowie Abs. 5 Satz 2 und 5 genannten Fristen sowie $\$ 60$ Abs. 1 des Ersten Buches entsprechend. Das Gesetz über das Verwaltungsverfahren der Kriegsopferversorgung ist entsprechend anzuwenden, soweit nicht das Zehnte Buch Anwendung findet. Die Auswirkungen auf die Teilhabe am Leben in der Gesellschaft werden als Grad der Behinderung nach Zehnergraden abgestuft festgestellt. Die Maßstäbe des $\$ 30$ Abs. 1 des Bundesversorgungsgesetzes und der aufgrund des $\$ 30 \mathrm{Abs}$. 17 des Bundesversorgungsgesetzes erlassenen Rechtsverordnung gelten entsprechend. Eine Feststellung ist nur zu treffen, wenn ein Grad der Behinderung von wenigstens 20 vorliegt. ..."

Das hier erwähnte „Bundesversorgungsgesetz" ist das Gesetz, das die Versorgung der Kriegsopfer regelt.

${ }^{22}$ ) „Kausales System“. 
kennzeichnet ( $\$ 3,84$ SGB IX), war diesem Schwerbeschädigtenrecht zunächst fremd. Ähnliches gilt für das Ziel umfassender Integration.

Verschiedene gesetzliche Ergänzungen schufen hier aber bereits in der Weimarer Zeit Abhilfe: es gab erste Ansätze zu Berufsfürsorge in der gesetzlichen UV und arbeitslosenversicherungsrechtliche Fördermaßnahmen für Behinderte.

Nach dem Ende der Nazizeit, die dem außer gewaltigen Problemen nichts hinzugefügt hatte, stand Deutschland wieder vor Aufgaben wie 1919, aber in weit größerem Umfang. Dies führte zum SchwerbeschädigtenG von 1953, das die Instrumente aus dem Recht der Weimarer Zeit übernahm und fortentwickelte, die herkömmlichen rechtlichen Strukturen in diesem Bereich aber auch dadurch verfestigte, dass es ausdrücklich am kausalen System der Schwerbehinderung und an der Beschränkung des Gesetzes auf deutsche Staatsangehörige festhielt.

Die Maßstäbe zur Bewertung von Behinderungen beruhten noch immer auf dem gleichen Schema. Das SchwerbehindertenG von 1974 führte dann zur Lösung vom kausalen Begriff der Schwerbehinderung unabhängig von der jeweiligen Ursache.

Auch wenn sich das rechtliche Umfeld des Begriffs der Schwerbehinderung schon bis dahin deutlich verändert hatte - neben verschiedenen Maßnahmen zur Rehabilitation wurden auch die Rechte der Vertrauensleute der Schwerbehinderten deutlich gestärkt und die Regeln über deren Zusammenarbeit mit den Betriebsräten spürbar verbessert -, so blieb es doch auch dann noch bei einem mehr oder weniger integrationsfernen Begriff der Behinderung. Ähnlich wie nach dem Ersten Weltkrieg war auch 1974 noch derjenige behindert, bei dem eine nicht nur vorübergehende Funktionsbeeinträchtigung vorlag, die auf einem regelwidrigen körperlichen, geistigen oder seelische Zustand beruht. Der Behinderungsbegriff enthielt somit keine integrationsbezogene Komponente. Und auch die für die Aufgaben des Schwerbehindertenrechts zuständige Behörde hieß damals noch immer nicht etwa Rehabilitationsamt oder gar Integrationsamt, sondern "Hauptfürsorgestelle“.

4. Diese dem Recht der Kriegsopferversorgung entnommenen Bewertungsregeln für Behinderungen und deren Schweregrad waren keine rechtlich festgelegten Maßstäbe, sondern versorgungsärztlich begründete Erfahrungssätze, die in ein seit dem Ersten Weltkrieg mehrfach überarbeitetes Werk namens „Anhaltspunkte für die ärztliche Gutachtertätigkeit im sozialen Entschädigungsrecht und nach dem Schwerbehindertenrecht" zusammengefasst worden waren.

Wie sich schon aus deren Bezeichnung ergibt, sind die "Anhaltspunkte ..." nicht an die Behörden gerichtet, die über die Einschätzung von Behinderungen zu entscheiden haben, sondern an die ärztlichen Gutachter. Ihr praktischer Wert bestand daher vor allem darin, dass durch ihre Verwendung eine weitgehend gleichmäßige Behandlung aller Betroffenen garantiert war. Das hat auch das BSG in einem Urteil aus dem Jahre 1997 so gesehen $^{23}$ ).

${ }^{23}$ ) BSG 9. 4. 1997 - 9 RVs 4/95

„... Der Grad der Behinderung ist ... unter Heranziehung der Anhaltspunkte für die ärztliche Gutachtertätigkeit ... in der jeweils geltenden Fassung festzulegen. Zwar beruhen die Anhaltspunkte weder auf dem Gesetz noch auf einer Verordnung oder auch nur Verwaltungsvorschriften, sodass sie keinerlei Rechtsqualität haben, dennoch sind sie
5. Man könnte also versucht sein, durch die Rechtsprechung zum Begriff der Behinderung die Bedeutung der Anhaltspunkte dem nunmehr maßgebenden Behinderungsbegriff des $\$ 2$ SGB IX ${ }^{24}$ ) anzupassen.

Dies erschien umso notwendiger, als auch die „Anhaltspunkte“ 2008 noch immer ausschließlich auf der Vorstellung zu beruhen scheinen, dass behindert ist, wer die genannten Funktionseinbußen aufweist, eine Berücksichtigung des nunmehr in \$2 SGB IX zusätzlich ins Gesetz eingefügten Kriteriums, dass auch solche Personen behindert sind, bei denen durch die Beeinträchtigung „...ihre Teilhabe am Leben in der Gesellschaft beeinträchtigt ist", fehlt dabei völlig.

Das ist auch nicht weiter verwunderlich, denn die Anhaltspunkte sind ja nicht für das moderne Recht der Rehabilitation und der weitgehenden Integration geschaffen worden, sondern für die Umsetzung der Vorgaben des Kriegsopferversorgungsrechts, und dabei ging es ja nicht um „Teilhabe am Leben in der Gesellschaft", sondern um „Fürsorge“.

6. An den Anhaltspunkten - und dem zugrundeliegenden Begriff einer Behinderung - wurde im Ubrigen auch nicht erst seit Inkrafttreten des SGB IX Kritik geübt; dass für die Beurteilung und Einstufung des Grades der Behinderung in Wahrheit noch immer allein die Beschädigung und nicht irgendwelche Auswirkungen auf die Fähigkeit zur Teilhabe ausschlaggebend waren, führte nicht nur dazu, dass die "Anhaltspunkte“ etwas verächtlich als "Knochentaxe" bezeichnet wurden - und werden -, sondern schon seit den späten 70er Jahren auch zu der Feststellung, dass hier eine Änderung der Einschätzung erforderlich sei.

7. Immerhin hat das BSG in einer Entscheidung von 1986 schon den Versuch unternommen, unter Einbeziehung der Auswirkungen des der Behinderung zugrundeliegenden Zustandes eine Weiterentwicklung des Behindertenbegriffs anzustoßen ${ }^{25}$ ). Obwohl das BSG die Bindung an die Anhaltspunkte verteidigt,

als antizipierte Sachverständigengutachten anzusehen, die in der Praxis wie Richtlinien für die ärztliche Gutachtertätigkeit wirken, deshalb normähnliche Auswirkungen haben und im Interesse einer gleichmäßigen Rechtsanwendung wie untergesetzliche Normen von den Gerichten anzuwenden sind ..."

${ }^{24)} \$ 2$ SGB IX:

"Behinderung.

(1) Menschen sind behindert, wenn ihre körperliche Funktion, geistige Fähigkeit oder seelische Gesundheit mit hoher Wahrscheinlichkeit länger als sechs Monate von dem für das Lebensalter typischen Zustand abweichen und daher ihre Teilhabe am Leben in der Gesellschaft beeinträchtigt ist. ..."

${ }^{25}$ ) Urteil 9. 10. 1987 - 9a RVs 5/86:

„... Nach dieser Definition versteht man unter Behinderung nicht den regelwidrigen körperlichen, geistigen oder seelischen Zustand als solchen. Vielmehr ist mit diesem Begriff die Auswirkung von Funktionsstörungen erfasst, die durch einen regelwidrigen Kör per-, Geistes- oder Seelenzustand verursacht wurden. Demgegenüber stellt der Beklagte allein auf die körperliche Regelwidrigkeit selbst ab, die die Salmonellendauerausscheidung sscheidung Bakterien ... ausgeschieden würden, seien keine weiteren Normabweichungen feststellbar; die fragliche Gesundheitsstörung führe nicht zu einer Schwächung des körperlichen Allgemeinzustandes und demgemäß auch nicht zu einer Funktions- bzw Leistungseinbuße. Der daraus gezogenen Schlussfolgerung des Beklagten, eine Behinderung liege demgemäß 
versucht es hier doch auch, den Begriff der Behinderung um den Blick auf die Auswirkungen des Beeinträchtigungszustandes auszuweiten; die weitere Konsequenz, dass auch eine eigenständige Bewertung dieser Auswirkungen auf die Fähigkeit zur Führung eines normalen und unabhängigen Lebens und zur völligen und gänzlichen Integration in die Gesellschaft ins Blickfeld zu nehmen wäre, ist allerdings noch nicht zu erkennen. Auf andere Weise nähert sich das BSG dem Problem der Modernisierung der Anhaltspunkte schließlich, wenn es in einer Entscheidung vom 24.4.2008 ${ }^{26}$ ) lapidar erklärt, das Ziel der Teilhabe an den verschiedenen Lebensbereichen sei nun in den Vordergrund gerückt, dies habe man allerdings auch schon nach altem Recht berücksichtigt.

8. ME kommt man nicht umhin, festzustellen, dass wir jetzt in $\$ 2$ des SGB IX einen sehr viel weitergehenden Begriff von Behinderung haben, nämlich angereichert durch die Formulierung „... und daher ihre Teilhabe am Leben in der Gesellschaft beeinträchtigt ist", und wir müssen zusätzlich feststellen, dass dies auf die „Anhaltspunkte“ bisher keine Auswirkungen gehabt hat.

Der Weg, die Modernisierung des Behinderungsbegriffs entsprechend den nunmehrigen Vorgaben des SGB IX durch eine flexiblere Rechtsprechung voranzutreiben, wäre daher verlockend gewesen, wollte man verhindern, dass die Normierung des zusätzlichen Begriffsmerkmals der Teilhabe am Leben in der Gesellschaft in $\$ 2$ SGB IX folgenlos bleibt.

9. Nun tritt aber noch eine weitere Schwierigkeit auf: Der seit langem beklagten Situation, dass in Gestalt der Anhaltspunkte die Rechtsprechung an Bewertungsregeln gebunden sein soll, die keine Rechtsqualität haben, ist nun zwar abgeholfen werden, aber nicht den sich aus dem Teilhabebegriff nunmehr ergebenden Folgerungen. Nach dem bereits zitierten $\$ 30$ Abs 1 Bundesversorgungsgesetz (BVG) in Verbindung mit dessen Abs 17 sind jetzt die Bewertungsmaßstäbe, die bisher nur als die beschriebenen „Anhaltspunkte“ ohne Rechtsnormqualität existierten, durch eine Rechtsverordnung geregelt werden.

Diese Verordnung ist zum 1.1.2009 in Kraft getreten. In dem Anhang zu deren $\$ 2$ sind die Bewertungsgrundsätze, die bisher in den "Anhaltspunkten“ enthalten waren, festgelegt worden. Wie es scheint, sind dabei aber die "Anhaltspunkte“ wie befürchtet „nahezu vollständig“ übernommen worden.

10. Dazu ist festzustellen, dass der im Hinblick auf den Begriff der Behinderung mit dem SGB IX vorgenommene „Paradigmenwechsel“ in Richtung auf eine Teilhabe am Leben in der Gesellschaft in den für die Beurteilung im Einzelfall geltenden Maßstäben nach wie vor keinen Niederschlag gefunden hat.

11. Zudem ist auch in Zukunft hier nicht unbedingt Abhilfe zu erwarten. Denn der in $\$ 3$ der neuen Verordnung vorgesehene Beirat zu Weiterentwicklung der Bewertungsmaßstäbe soll so zusammengesetzt sein, dass er zwar medizinisches Fachwissen vermitteln kann, nicht aber auch diejenigen Erkenntnisse, die erforderlich wären, die Auswirkungen einer Beeinträchtigung auf die Fähigkeit

nicht vor, ist nicht beizupflichten. Darauf hat auch das Aktionsprogramm des Rates der Europäischen Gemeinschaft abgehoben, wenn als Ziel der Behindertenhilfe ua herausgestellt ist, dass „die Behinderten zur Führung eines normalen und unabhängigen Lebens zu befähigen und voll und ganz in der Gesellschaft zu integrieren sind".

26) B 9/9a SB $10 / 06 \mathrm{R}$. zur „Teilhabe am Leben in der Gesellschaft" unter soziologischen und psychotherapeutischen Aspekten richtig einzuschätzen.

12. Man darf daher wohl folgendes Fazit ziehen: Akzeptiert man die Verschiedenheit der Begriffe „Behinderung“ zum einen im Versorgungsrecht und zum anderen im Recht der Rehabilitation, so steckt in der Verwendung eines einheitlichen Bewertungsmaßstabes für beide Begriffe ein Widerspruch.

Man sollte daher in Betracht ziehen, ob hier nicht zwei unterschiedliche Bewertungssysteme entwickelt werden sollten. Der Hinweis darauf, dass sich das Überkommene doch bewährt habe, wird nicht genügen. 